San Jose State University

SJSU ScholarWorks

Master's Theses

Master's Theses and Graduate Research

Fall 2017

\title{
Life History Aspects and Taxonomy of Deep-Sea Chondrichthyans in the Southwestern Indian Ocean
}

Paul Joseph Clerkin

San Jose State University

Follow this and additional works at: https://scholarworks.sjsu.edu/etd_theses

\section{Recommended Citation}

Clerkin, Paul Joseph, "Life History Aspects and Taxonomy of Deep-Sea Chondrichthyans in the Southwestern Indian Ocean" (2017). Master's Theses. 4869.

DOI: https://doi.org/10.31979/etd.ms3e-x835

https://scholarworks.sjsu.edu/etd_theses/4869

This Thesis is brought to you for free and open access by the Master's Theses and Graduate Research at SJSU ScholarWorks. It has been accepted for inclusion in Master's Theses by an authorized administrator of SJSU ScholarWorks. For more information, please contact scholarworks@sjsu.edu. 
LIFE HISTORY ASPECTS AND TAXONOMY OF DEEP-SEA CHONDRICHTHYANS IN THE SOUTHWESTERN INDIAN OCEAN

\author{
A Thesis \\ Presented to \\ the Faculty of Moss Landing Marine Laboratories and \\ San José State University
}

\author{
In Partial Fulfilment \\ of the Requirements for the Degree \\ Master of Science
}

by

Paul J. Clerkin

December 2017 
(C) 2017

Paul J. Clerkin

ALL RIGHTS RESERVED 
The Designated Thesis Committee Approves the Thesis Titled

LIFE HISTORY ASPECTS AND TAXONOMY OF DEEP-SEA CHONDRICHTHYANS IN THE SOUTHWESTERN INDIAN OCEAN

by

Paul J. Clerkin

APPROVED FOR THE DEPARTMENT OF MARINE SCIENCE SAN JOSÉ STATE UNIVERSITY

December 2017

Dr. David A. Ebert

Dr. Scott Hamilton

Dr. Kenneth H. Coale
Moss Landing Marine Laboratories

Moss Landing Marine Laboratories

Moss Landing Marine Laboratories 


\begin{abstract}
ASPECTS OF THE LIFE HISTORY AND TAXONOMY OF DEEP-SEA CHONDRICHTHYANS IN THE SOUTHWESTERN INDIAN OCEAN

by Paul J. Clerkin
\end{abstract}

During the last quarter of a century, the conservation and management of chondrichthyans (collectively, sharks, rays, and chimaeras) has received considerable focus. This is especially true for deep-sea chondrichthyans. As technologically advanced fisheries expand into deeper waters of the high seas, new chondrichthyan species are being discovered and described at an increasing rate. The objectives of this study were to investigate the deep-sea chondricthyan fauna in a remote region of the Southwestern Indian Ocean Offshore and provide descriptions of three species of Chimaeridae previously unknown to science, and collect and analyze biological parameters relating to the life histories of all shark species encountered. Specimens were collected as bycatch in deep-sea fisheries from 46 sites along deepwater seamounts of the Southwest Indian Ocean Ridge and the Madagascar Ridge. Among the species encountered were three relatively large chimaeroids which, upon closer examination, were determined to be distinct from all other known members of the family. A description these three new species is presented. A total of 4009 specimens were examined and sex ratios, size range, smallest mature, largest immature, and length at $50 \%$ maturity $\left(L_{T 50}\right)$ calculated. Detailed information is presented on the reproductive biology, life history, and distribution of 31 species representing 14 genera. 


\section{ACKNOWLEDGEMENTS}

This study was supported by the National Science Foundation's Jaws and Backbone: Chondrichthyan Phylogeny and a Spine for the Vertebrate Tree of Life, grant: DEB 01132229 to Gavin Naylor, Discovery Communications, Save Our Seas Foundation, the Food and Agriculture Organization (United Nations), Dr. Earl H. Myers and Ethel M. Myers Oceanographic and Marine Biology Trust, the Lerner Gray Memorial Fund, and the COAST Travel Grant.

I whole heartedly thank my thesis committee members Drs. David A. Ebert, Scott Hamilton, and Kenneth H. Coale. The knowledge, guidance, and support you have provided has been beyond measure. The opportunities and resources that you provide your students are signposts of excellence that defines the MLML experience. Your passion for your disciplines and your dedication to your students are inspirational. You have had a valuable role in shaping me as a scientist, and for that I am grateful. Thank you.

I sincerely thank Dr. Ross Shotton, Executive Secretary, Southern Indian Ocean Deepsea Fishers Association for his efforts in securing the collaboration of the project's many participants, the Sealord Corporation of New Zealand for generously enrolling its F/V Will Watch in support of project goals, and the captains, officers, and crew of the Will Watch for their openhearted hospitality and tireless efforts on behalf of my research.

I am grateful to Daroomalingum Mauree, Director of Fisheries, Republic of Mauritius and to Boodhun Ramcharrun, Scientific Officer, Mauritius Ministry of Fisheries for their kind facilitation of our work at the Albion Fisheries Research Centre. I thank my island 
brothers, Rajiv Bacorisen, Asaid Morrarby, Nankishore Toofany, and everyone at the Albion Fisheries Research Centre whose daily assistance on Mauritius was crucial to the completion of this project.

I am indebted to Dr. Dominique A. Didier of Millersville University for her expertise in the confirmation of species identification and guidance on expanding chimaeroid morphometric measurements. I thank Edoardo Mostarda, Jessica Sanders, and Merete Tandstad of the United Nation's Food and Agriculture Organization for their role in the planning and support of these surveys.

I thank G.J.P. Naylor for overall project support and Jenny Kemper of the Hollings Marine Laboratory at the College of Charleston for sequencing genetic material of this study. For their reliable assistance in obtaining morphometric measurements, recording data, and for helping with many other project related tasks at Moss Landing Marine Laboratories, I thank the "Shark Army” Jessica Bean, Madeleine Harris, Matthew Lawson, Marty Schmidt and Jimmy Yun. I extend my gratitude to Dr. William Head, Dr. Gerick Bergsma, Matthew Subia, Robert Quiñonez, and all the people with California State University at Monterey Bay’s Undergraduate Research Opportunities Center (UROC) for arranging the help of dedicated interns.

I sincerely thank the following individuals for their support on various parts of this study: R. Downie (CSIRO Oceans and Atmosphere Flagship, Hobart, Tasmania, Australia), D. Catania and J. Fong (California Academy of Sciences), D. Clarke and M. Bougaardt W. Florence, E. Hoenson, and C. Untiedt (South African Museum), R. Bills, E. Heemstra, and Rob Leslie (South African Institute of Aquatic Biodiversity), R. 
Leslie and L. Singh (Department of Agriculture, Forestry and Fisheries, South Africa), Samuel Iglésias and Elena Luchetti (National Museum of Natural History, Paris).

A large portion of the data for this project was analyzed in MATLAB with the aid and patience of Stephanie Flora. Thank you Stephanie for only rarely enforcing your one dollar per question policy. Further, I thank all my friends at Moss Landing Marine Laboratories for surrounding me with a supportive collegial community. In particular, I thank the PSRC for its support and camaraderie, with special thanks to Jenny Bigman, Justin Cordova, Kelsey James, Jessica Jang, Matthew Jew, James Knuckey, Breanna Machuca, Melissa Nehmens, Amber Reichert, Kelley van Hees, and Victoria Vasquez. I would like to thank Kristin Walovich for her contribution of comparative measurements used in chapter one.

I also thank my lifelong friends Jerry Doud, Enissan Martinez, and Kyle Moreno for helping me stay grounded throughout this process. I thank Stephen Loiacono for his much appreciated advice and consultation over the years, Joseph Manzo for his support and patience.

Finally, I realize I never would have been able to accomplish this project without the unconditional love and support of my family and for that I thank my parents, John and Imelda, and my siblings John, Cathleen, Michael, and Suzanne. With that in mind, I dedicate my thesis to my Mom who always allowed me to dream and to my Dad who always told me I could do anything. I believe my curiosity and creativity are derived from my Mom who has done her best to avoid growing up. I credit my relentlessness and compassion to my dad who has never encountered a problem he couldn't s solve, nor has 
ever been too tired to help family members when they needed him. Thanks Mom and Dad for your wisdom and guidance, and for your constant support and, unwavering faith in me. 


\section{TABLE OF CONTENTS}

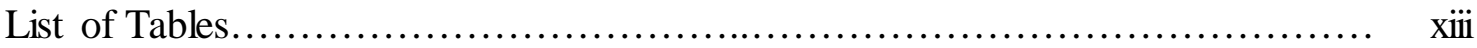

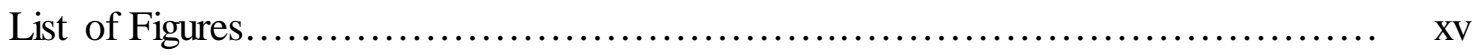

General Introduction....................................................... 1

General Materials and Methods................................................ 10

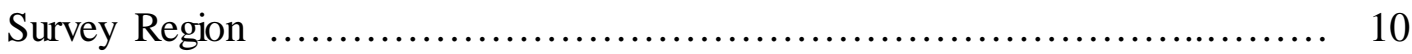

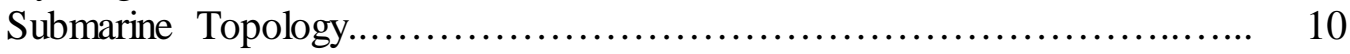

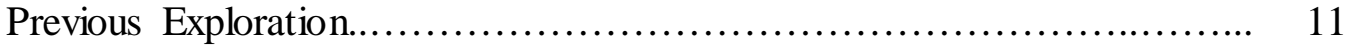

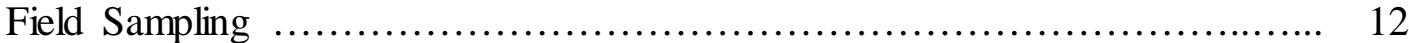

Study Site and Field Seasons........................................ 12

Specimen Collection...................................................... 12

Chapter One: New species of Chimaera (Chondrichthyes: Holocephali:

Chimaeriformes: Chimaeridae) from the Southwestern Indian Ocean ............... 15

Introduction....................................................... 15

Material and Methods .................................................... 16

Study Location................................................... 16

Specimen Collection................................................. 17

Measurements/Meristics.......................................... 17

Cluster Analysis. .................................................. 22

Genetic Analysis................................................. 23

Institutional Acronyms................................................ 24

Chimaera willwatchi, sp. nov ........................................... 24

Holotype......................................................... 24

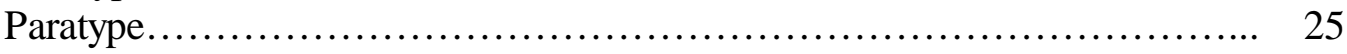

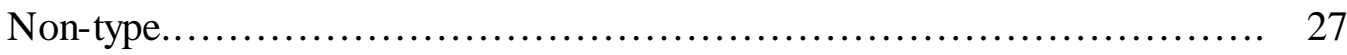

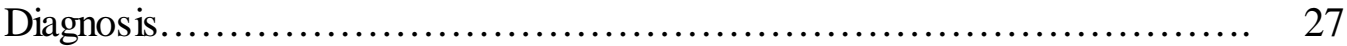

Description......................................................................................... 29

Ontogenetic Differences Between Large and Small Specimens.............. 38

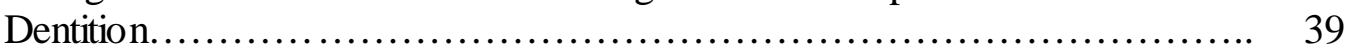

Coloration.............................................................. 39

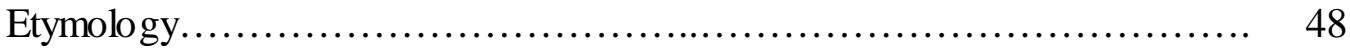

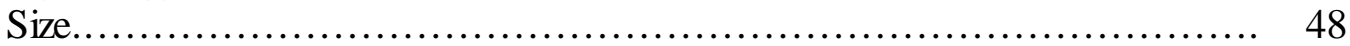

Distribution.......................................................... 48

Biological Notes.................................................... 48

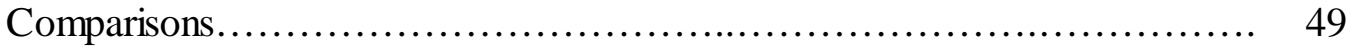

Chimaera didierae, sp. nov …............................................ 55

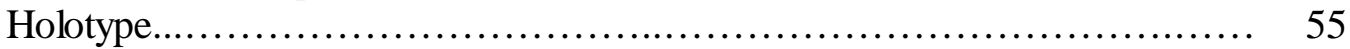

Diagnosis.............................................................. 55

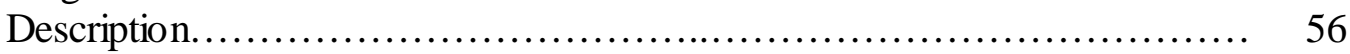




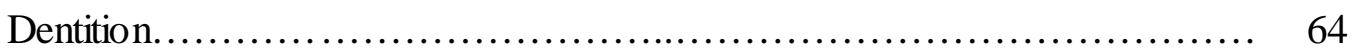

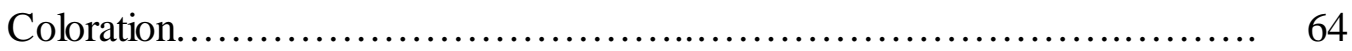

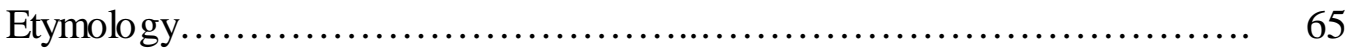

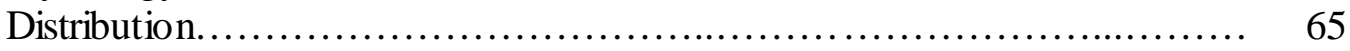

Biological Notes................................................. 66

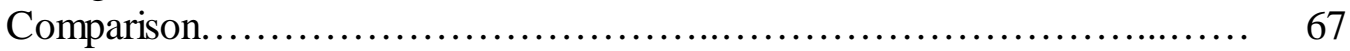

Chimaera buccanigella, sp. nov ....................................... $\quad 70$

Holotype......................................................... 70

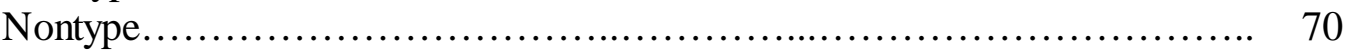

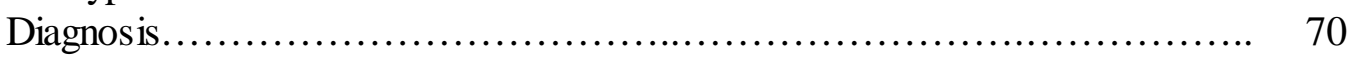

Description............................................................ 71

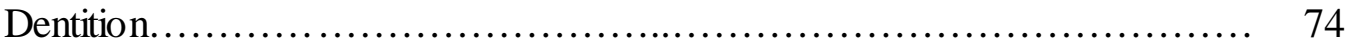

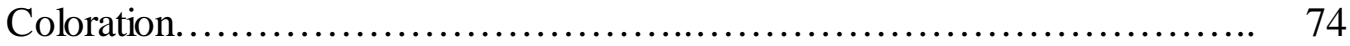

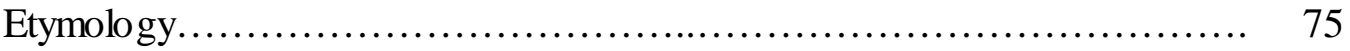

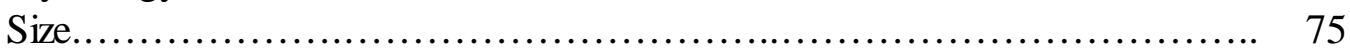

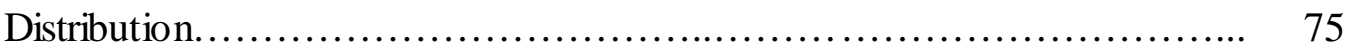

Biological Notes.................................................. 75

Comparison .......................................................... $\quad 75$

Comparison of New Southwestern Indian Ocean Chimaera Species.............. 78

Discussion.............................................................. 80

Key to Indian Ocean Chimaera species ..................................... 81

Chapter Two: The Natural and Life Histories of Deep-sea Chondrichthyans in the

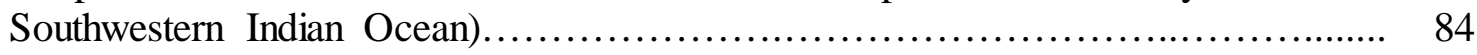

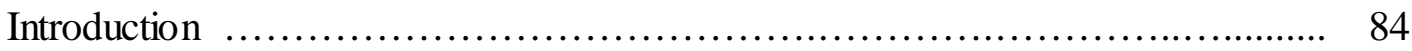

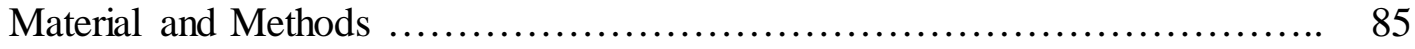

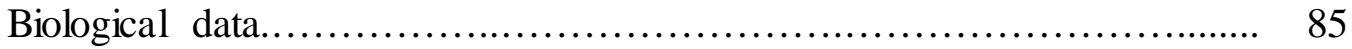

Maturity Determination................................................. 86

Sexual Dimorphism. .............................................. 99

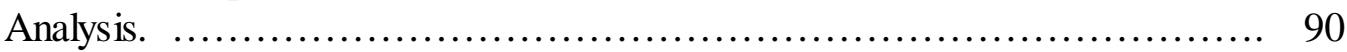

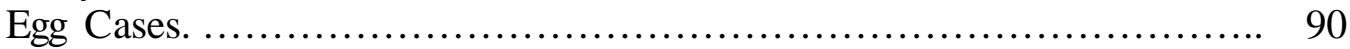

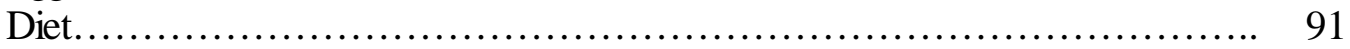

Distribution............................................................. 91

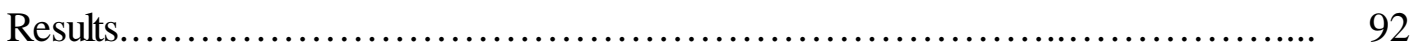

Centrophorus granulosus.............................................. 92

Centrophorus squamosus............................................. 99

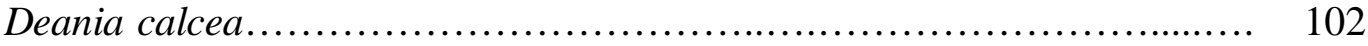

Deania profundorum................................................... 104

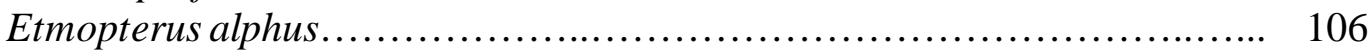

Etmopterus bigelowi................................................ 107

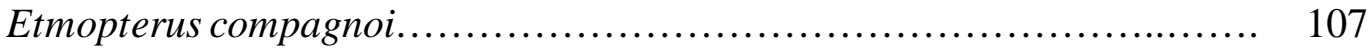

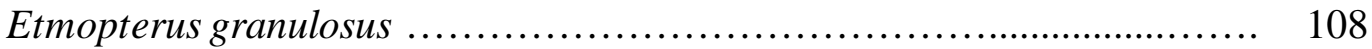

Etmopterus pusillus................................................. 111

Etmopterus sculptus................................................. 113 
Etmopterus cf. sculptus ................................................. 113

Scymnodon plunketi ................................................. 113

Centroscymnus coelolepis................................................ 116

Centroscymnus owstonii ........................................... 119

Centroselachus crepidater ............................................... 121

Zameus squamulosus ................................................. 123

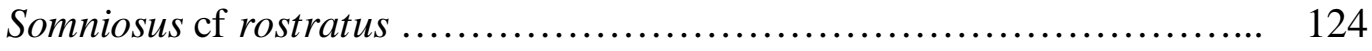

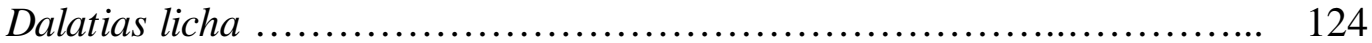

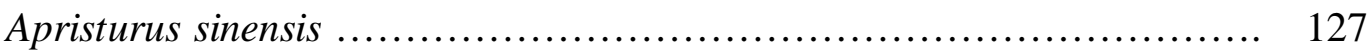

Apristurus cf. albisoma ............................................... 130

Apristurus cf. ampliceps 1.............................................. 131

Apristurus cf. ampliceps 2................................................ 131

Apristurus cf. manis ..................................................... 132

Apristurus cf. melanoasper .................................................. 133

Bythaelurus bachi .................................................. 134

Bythaelurus naylori ................................................... 136

Pseudotriakis microdon................................................. 137

Chimaera willwatchi .................................................. 140

Chimaera didierae ................................................ 143

Chimaera buccanigella................................................. 143

Hydrolagus sp. A .................................................. 143

Discussion............................................................... 143

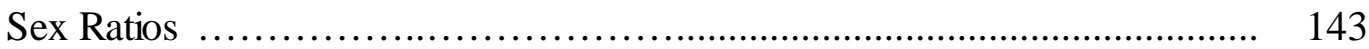

Maximum Length ...................................................... 145

Sexual Dimorphism ............................................... 146

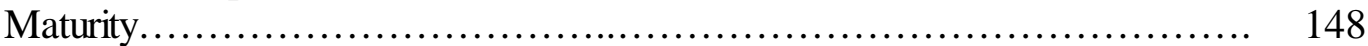

Mature Oocyte Number............................................. 149

Fecundity....................................................... 150

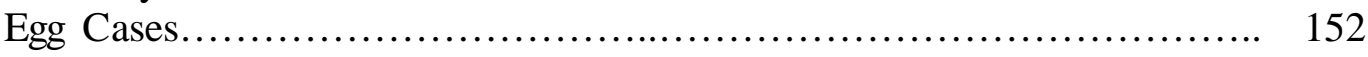

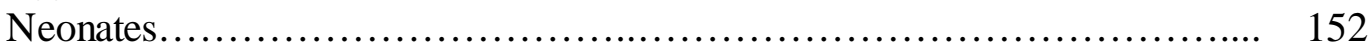

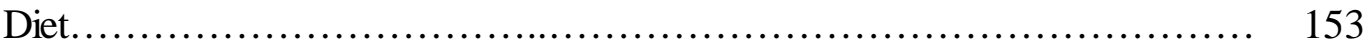

Distribution......................................................... 155

Life Strategy and Reproductive Mode................................. 160

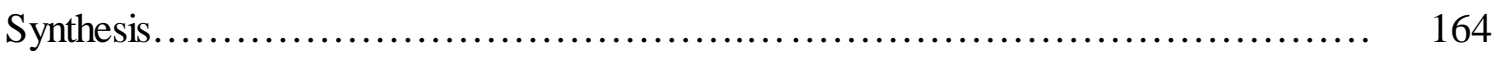

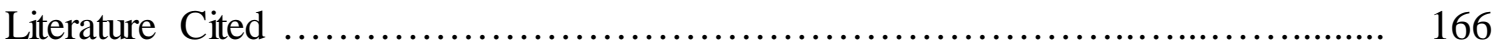

Appendices................................................................ 184

Appendix A: Definition and explanation of chimaeroid morphometric

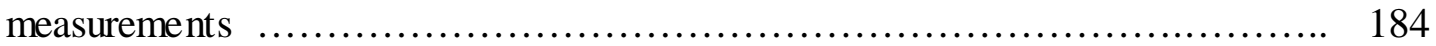

Appendix B: Locality, tissue identification number, voucher specimen collection number, and GenBank accession number for samples used in genetic analysis 
Appendix C: Comparative Material...................................... 190 


\section{LIST OF TABLES}

Table 1. SIMPER analysis of morphometric measurements defining Chimaera willwatchi, displaying the top fifteen ranked morphometric measurements (all with contributing percentages above 3.00) which distinguish Chimaera willwatchi as a species. The contributing percentages are expressed as \%........

Table 2. Morphometric measurements of body presented as body length proportions (\%BDL) of Chimaera willwatchi, sp. nov. holotype, paratypes, and nontypes measuring $<300 \mathrm{~mm}$. Holotype was a mature male, paratypes included eight males and seven females, and nontypes included 10 males and 30 females.

Table 3. Morphometric measurements of head presented as body length proportions (\%BDL) of Chimaera willwatchi, sp. nov. holotype, paratypes, and nontypes measuring $<300 \mathrm{~mm}$. Holotype was a mature male, paratypes included eight males and seven females, and nontypes included 10 males and 30 female.

Table 4. Morphometric measurements of body presented as body length proportions (\%BDL) of Chimaera willwatchi, sp. nov. nontype morphotypes B (two specimens) and C (six specimens)....

Table 5. Morphometric measurements of head presented as body length proportions (\%BDL) of Chimaera willwatchi, sp. nov. Nontype morphotypes B (two specimens) and $\mathrm{C}$ (six specimens).

Table 6. SIMPER pairwise comparison of morphometric measurements. Displaying the top five ranked morphometric measurements for each species comparison; the contributing and cumulative percentages are expressed as $\%$

Table 7. Morphometric measurements of body presented as body length proportions (\%BDL) of Chimaera didierae, sp. nov., holotype CAS 242334, immature female, and Chimaera buccanigella, sp. nov., holotype CAS 242335, immature female.

Table 8. Morphometric measurements of head presented as body length proportions (\%BDL) of Chimaera didierae, sp. nov., immature female, and Chimaera buccanigella, sp. nov., immature female. 
Table 9. A list of species encountered, the relationship between total length (TL) and length at first maturity, length at first maturity in relation to maximum length ( $L_{T \max }$ ), and length at $50 \%$ maturity ( $\left.L_{\mathrm{T} 50}\right)$ for a) Squaliformes:

Centrophoridae, Etmopteridae, Somniosidae, Dalatiidae, b)

Carcharhiniformes: Scyliorhinidae, Pseudotriakidae, and Holocephali:

Chimaeridae; and lengths reported in the literature for 31 species of deep-sea chondricthyans encountered in the SWIO, c) Centrophoridae, Etmopteridae, and d) Somniosidae, Dalatiidae, scyliorhinids and, Pseudotriakidae.....

Table 10. Sex ratio significance evaluated by $p$-value $<0.05$, and $\chi^{2}$ value for overall, adult, and subadult sex ratio.

Table 11. List of numbers of ovarian oocytes (average and max), uterine eggs (left max, right max, total max, and average), and max width of oocytes.

Table 12. List of numbers species bearing pups, number of pups, and number of pups reported in literature.

Table 13. List of species found with stomach contents, prey items given by number and percentage composition of estimated volume and diet reported in the literature....

Table 14. Distribution of species encountered during surveys and their reported ranges

Table 15. SIMPER pairwise comparison of species composition between regions. The contributing and cumulative Bray Curtis similarity (species contributions) percentages are expressed as \%

Table 16. SIMPER pairwise comparison of species composition between trawl gear types. The contributing and cumulative Bray Curtis similarity (species contributions) percentages are expressed as \%.... 


\section{LIST OF FIGURES}

Figure 1. Map of the Southwestern Indian Ocean showing a) Madagascar Ridge, with b) the northern region, c) southern region (Walters Shoal), and d) the Southwest Indian Ocean Ridge. Trawl locations indicated by green circles; 1 March to 23 April 2012 and 10 April to 7 June 2014.

Figure 2. Diagram of lengths (blue) and paired fin measurements (red). See Appendix A for definition and explanation of abbreviations

Figure 3. Diagram of measurements: a) across body (blue), inter fin spaces (red), b) body heights (blue), unpaired fin heights and margins (red), and overlap (green). See Appendix A for definition and explanation of abbreviations.

Figure 4. Diagram of head measurements: lengths (blue), widths (red), direct distance (green), eyes (yellow), height (purple). See Appendix A for definition and explanation of abbreviations

Figure 5. Diagram of canals of the head (blue) and canal measurements (red). See Appendix A for definition and explanation of abbreviations.

Figure 6. Full body lateral view of Chimaera willwatchi, sp. nov., holotype CAS 242336, mature male $834 \mathrm{~mm}$ TL, $492 \mathrm{~mm}$ BDL, A) Photograph, B) Illustration

Figure 7. Chimaera willwatchi, sp. nov., holotype CAS 242336, mature male $834 \mathrm{~mm}$ TL, 492 mm BDL illustrations of a) Frontal tenaculum, b) Lateral view of pelvic claspers, c) Pre-pelvic tenacula, d) Tooth plates, and e) Skeletonized skull

Figure 8. Illustration of Chimaera willwatchi, sp. nov., lateral lines of head showing: infraorbital to angular canal (IOA), junction of the oral-infraorbital canal to junction of the oral and angular canal, preopercular to main trunk (OTM), preopercular canal (POP), and oral canal (O).

Figure 9. Map of study area denoting where Chimaera willwatchi, sp. nov., specimens were collected, with subpopulations represented by circles (morph A), star (morph B), and triangles (morph C)...

Figure 10. Chimaera willwatchi, sp. nov., morph A: a) holotype CAS 242336, mature male $834 \mathrm{~mm}$ TL, $492 \mathrm{~mm}$ BDL, b) paratype, immature male, $620 \mathrm{~mm}$ TL, $323 \mathrm{~mm}$ TL, c) paratype, newly hatched female, CAS 242351, $290 \mathrm{~mm}$ TL, $117.58 \mathrm{~mm}$ BDL..................................................... 
Figure 11. Chimaera willwatchi, sp. nov., morph B: a) non-type CAS, immature male, 694 mm TL, 369 mm BDL, b) non-type CAS 242354, immature female, $477 \mathrm{~mm}$ TL, 252mm BDL...........................................

Figure 12. Chimaera willwatchi, sp. nov., morph C: a) non-type CAS 242358, immature female, $975 \mathrm{~mm}$ TL, $542 \mathrm{~mm}$ BDL, b) non-type CAS 242342, immature female, 620mm TL, $323 \mathrm{~mm}$ BDL

Figure 13. Maximum likelihood tree topology based on a general-time reversible (GTR) substitution model + gamma distribution for Chimaera willwatchi, sp. nov., Chimaera didierae, sp. nov., Chimaera buccanigella, sp. nov., and comparative species based on aligned NADH2 DNA sequences. GenBank accession numbers follow species (see Appendix B). Bootstrap support values of main clades shown on tree. Specimens indicated in bold are designated holotypes.

Figure 14. Normalized Euclidean distances illustrating morphometric dissimilarities between 10 chimaeroid species

Figure 15. Full body lateral Chimaera didierae, sp. nov., holotype CAS 242334, immature female, $890 \mathrm{~mm}$ TL, $704 \mathrm{~mm}$ BDL, $532 \mathrm{~mm}$ PCL a) photograph, b) illustration.

Figure 16. Illustration of Chimaera didierae, sp. nov., lateral lines of head showing: infraorbital to angular canal (IOA), junction of the oral-infraorbital canal to junction of the oral and angular canal, preopercular to main trunk (OTM),

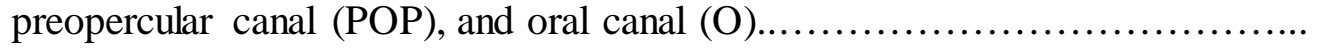

Figure 17. Map of study area denoting collection sites of Chimaera didierae, sp. nov., (yellow triangle), and Chimaera buccanigella, sp. nov. (red circle)............

Figure 18. Full body lateral photograph of Chimaera buccanigella, sp. nov., a) Holotype CAS 242335, immature female, $830 \mathrm{~mm}$ TL, $397 \mathrm{~mm}$ BDL, b) Illustration of holotype, c) Anterior view of holotype highlighting dusky mouth.

Figure 19. Illustration of Chimaera buccanigella, sp. nov., lateral lines of head showing: infraorbital to angular canal (IOA), junction of the oral-infraorbital canal to junction of the oral and angular canal, preopercular to main trunk (OTM), preopercular canal (POP), and oral canal (O) ..........................

Figure 20. Illustration of maturity ranking system for sharks a) males, b) females 87 
Figure 21. Illustration of maturity ranking system for chimaeroids showing stages of development of a) frontal tenaculum, b) pre-pelvic tenaculum, c) pre-pelvic claspers, and d) anal pad

Figure 22. Diagram of egg case measurements: egg case length (ECL), anterior border width (AWB), anterior respiratory fissure length (AFL), anterior width (AW), egg case height $(\mathrm{HI})$, posterior border width (PBW), posterior respiratory fissure length (PFL), posterior width (PW), waist width (WW), and flange height $(\mathrm{FH})$, and normalized as a percentage of ECL............................

Figure 23. Centrophorus granulosus: a) size distribution of males, b) relationship between inner clasper length (\%LT) and LT (males), c) size distribution of females, d) relationship between shell gland width (\%LT) and LT (females).

Figure 24. Distribution of the family Centrophoridae of this study: Centrophorus granulosus (red circle), Centrophorus squamosus (green square), Deania calcea (orange triangle), and Deania profundorum (purple star)

Figure 25. Centrophorus squamosus: a) size distribution of males, b) relationship between inner clasper length (\%LT) and LT (males).

Figure 26. Pie chart of prey items presented as percent composition of item-count for each species encountered with stomach contents. Broad taxonomic groups are shown as: cephalopod (red), fish (blue), crustacean (pink), mammal (orange), shark (grey), and unidentified (black).

Figure 27. Deania calcea: a) size distribution of males, b) size distribution of females, c) relationship between shell gland width (\%LT) and LT (females)

Figure 28. Deania profundorum: a) size distribution of females, b) relationship between shell gland width (\%LT) and LT (females)....

Figure 29. Distribution of the family Etmopteridae (sans E. granulosus. See figure 11): Etmopterus alphus (red circle), E. bigelowi (green square), E. compagnoi (orange triangle), E. pusillus (purple star), E. sculptus (yellow pentagon), E. cf. sculptus (maroon diamond)

Figure 30. Etmopterus granulosus: a) size distribution of males, b) relationship between inner clasper length (\%LT) and LT (males), c) size distribution of females, d) relationship between shell gland width (\%LT) and LT (females), distribution of males (red circles) and females (green circles) 
Figure 31. Scymnodon plunketi: a) size distribution of males, b) relationship between inner clasper length (\%LT) and LT (males), c) size distribution of females, d) relationship between shell gland width (\%LT) and LT (females)...............

Figure 32. Distribution of the family Somniosidae (sans Centroscymnus, Centroselachus, and Dalatias): Scymnodon plunketi (red circle), Zameus squamulosus (yellow pentagon), and Somniosus cf rostratus (blue hexagon)

Figure 33. Centroscymnus coelolepis: a) relationship between inner clasper length (\%LT) and LT (males), b) size distribution of females, c) relationship between shell gland width (\%LT) and LT (females).

Figure 34. Distribution of the genus Centroscymnus: C. coelolepis (green triangle) and C. owstonii (red circle).

Figure 35. Centroscymnus owstonii: a) size distribution of males, b) relationship between inner clasper length (\%LT) and LT (males), c) size distribution of females, d) relationship between shell gland width (\%LT) and LT (females).

Figure 36. Centroselachus crepidater: a) size distribution of males, b) relationship between inner clasper length (\%LT) and LT (males), c) size distribution of females, d) relationship between shell gland width (\%LT) and LT (females), e) distribution of males (red circles) and females (green circles)

Figure 37. Dalatias licha: a) size distribution of males, b) relationship between inner clasper length (\%LT) and LT (males), c) size distribution of females, d) relationship between shell gland width (\%LT) and LT (females), Distribution specimens encountered (red circles) and Haul 183 (green circle)

Figure 38. Apristurus sinensis: a) size distribution of males, b) relationship between inner clasper length (\%LT) and LT (males), c) size distribution of females.....

Figure 39. Distribution of the genus Apristurus: A. sinensis (red circle), A. cf. albisoma (green square), A. cf. ampliceps 1 (orange triangle), A. cf. ampliceps 2 (yellow pentagon), A. cf. manis (blue hexagon), and A. cf. melanoasper (maroon diamond)

Figure 40. Apristurus cf. manis: a) size distribution of males, b) relationship between inner clasper length (\%LT) and LT (males), c) size distribution of females..... 
Figure 41. Bythaelurus bachi: a) size distribution of females, b) left egg case removed from paratype, CAS 241443, adult female, $405 \mathrm{~mm}$ TL

Figure 42. Distribution of the genus Bythaelurus: B. bachi (Southwest Indian Ocean Ridge) and B. naylori (Walters Shoal).

Figure 43. Bythaelurus naylori: size distribution of males, b) relationship between inner clasper length (\%LT) and LT (males), c) size distribution of females, and d) egg case removed from a non-type female $452 \mathrm{~mm}$ TL.........................

Figure 44. Pseudotriakis microdon: a) size distribution of males, b) relationship between inner clasper length (\%LT) and LT (males), c) size distribution of females, d) relationship between shell gland width (\%LT) and LT (females), e) reproductive track of pregnant female with term pup, f) non-term embryo $69.1 \mathrm{~cm} L_{\mathrm{T}}$, g) term embryo $124.4 \mathrm{~cm} L_{\mathrm{T}}$, distribution of males (green circles), and females (red circles)....

Figure 45. Chimaera willwatchi: a) size distribution of males, b) relationship between inner clasper length (\%LT) and LT (males), c) size distribution of females.

Figure 46. Distribution of Chimaera encountered: Chimaera willwatchi (red circles), Chimaera didierae (green square), Chimaera buccanigella (orange triangle), Hydrolagus species A (yellow diamonds).

Figure 47. Plot of nMDS analysis illustrating differences in species composition of gear types. 


\section{General Introduction}

Chondrichthyans are among our oceans’ most successful groups of fishes, and are widespread as top predators in virtually every marine ecosystem (Ebert and Winton, 2010; White and Kyne, 2010; Ebert, 2013). Almost half (46.3\%) of all known chondrichthyans inhabit coastal waters of continental shelves, but only 3.7\% populate the neritic zone, where light penetrates to the sea floor, and epipelagic zone, where light is sufficient to sustain photosynthesis (Ebert and Winton, 2010; Steven, 2010; Dulvy et al., 2014). Fewer than $1 \%$ of chondrichthyans live in the twilight of the mesopelagic zone, and 3.2\% are freshwater obligate (Ebert and Winton, 2010; Stevens, 2010; Dulvy et al., 2014; Kyne \& Simpfendorfer 2010). Chondrichthyans also have penetrated into the harsh conditions of the deep-sea, with virtually half (46.0\%) of known species occurring below the photic zone of continental and insular slopes as well as on the abyssal plains and associated canyon ridges and seamounts (Kyne and Simpfendorfer, 2010; Dulvy et al., 2014; Rigby and Simpfendorfer, 2014).

Twelve of the 14 orders of chondrichthyans are represented in the deep-sea, a region defined by Rigby and Simpfendorfer (2014) as depths beyond the photic zone, and commonly estimated to begin 200 m below the ocean surface (Kyne and Simpfendorfer, 2010). The deep-sea is habitat to $52.7 \%$ of known shark species, $38.2 \%$ of batoid species, and 88.9\% of holocephalan species (Kyne and Simpfendorfer, 2010; Dulvy et al., 2014). These deepwater chondrichthyans represent $85.7 \%$ of chondrichthyan orders, 36 (60.0\%) chondrichthyan families, and 58 (56.1\%) known chondricthyan genera (Kyne 
and Simpfendorfer, 2010; Ebert, 2013, 2014; Rigby and Simpfendorfer, 2014; Eschmeyer et al., 2016).

Presently, there are over 1200 described species of extant chondrichthyans, a figure that includes 516 sharks, 633 valid batoids (Kyne and Simpfendorfer, 2010; Ebert et al., 2013; Ebert, 2013; Eschmeyer et al., 2016; Last et al., 2016), and 51 holocephalans (Eschmeyer and Fong, 2014; Last et al., 2016; Eschmeyer et al., 2017; Walovich et al., 2017). The number of valid chondrichthyan species is constantly increasing as new species are described. In 2016, a total of 37 new species of Chondrichthyans were described, and as of June 2017, nine more new species, including a new holocephalan have been named. Notably, many new chondrichthyan species are being discovered in remote, deep-sea habitats (Compagno, 1990; White and Last, 2012).

A recent study by Dulvy et al. (2014) assessed the conservation status of 1,041 chondrichthyan species as designated by the International Union for Conservation of Nature (IUCN). That study revealed that nearly one-quarter (249 species or $24 \%$ ) of all assessed chondrichthyans were Threatened, and nearly half (487 species or $46.8 \%$ ) were categorized as Data Deficient (Heupel and Simpfendorfer, 2010; Simpfendorfer et al., 2011; Dulvy et al., 2014). More than half (56.7\%) of these Data Deficient species inhabit the deep-sea.

The large number of chondrichthyans designated as Data Deficient by IUCN criteria highlights the extent to which basic biological knowledge as well as a fundamental understanding of life-history characteristics is lacking for these species (White and Last, 2012; Rigby and Simpfendorfer, 2014). The evaluation of species productivity is heavily 
based on life-history traits (Simpfendorfer et al., 2011; White and Last, 2012), and the absence of such data for deep-sea sharks results in the majority of knowledge being sourced from near-shore and pelagic chondrichthyan species (Rigby and Simpfendorfer, 2014).

Concern for the conservation and management of chondrichthyans, especially deepsea forms, has emerged over the past two decades (Stevens et al., 2000; Simpfendorfer and Kyne 2009; Kyne and Simpfendorfer, 2010). Although large variation in the group exists, cartilaginous fishes typically exhibit slower life histories compared to other vertebrate groups (Holden, 1974; White and Kyne, 2010; Hutchings et al., 2012) with many members characterized by slow growth, large body size, late onset of maturity, and few, well-developed offspring, each of which has a relatively high survivorship of reaching adulthood (Cortes, 2000; Stevens, 2000; Garcia et al., 2008; Simpfendorfer and Kyne, 2009; White and Last, 2012).

Recent awareness of the high potential extinction risk of chondrichthyan species has sparked an international effort for the sustainable management of these fishes (Garcia et al., 2008; Rigby and Simpfendorfer, 2014). The Food and Agriculture Organization (FAO) of the United Nations highlighted this concern with the publication of the International Plan of Action (FAO-IPOA, 1999). In an attempt to fill in major gaps in our knowledge of the life-history traits of cartilaginous fishes, the United Nations Food and Agriculture Organization (FAO) has encouraged voluntary participation by all states involved in shark catches (as target or bycatch) in its International Plan of Action for the Conservation and Management of Sharks (IPOA-Sharks) (FAO, 2010-2014). Informed 
management practices must rest on sound taxonomy and accurate life-history data (Simpfendorfer et al., 2011; White and Last, 2012).

A major threat confronting chondrichthyans emanates from increased fishing pressure for both targeted catch and bycatch (Stevens, 2000; Dulvy et al., 2014). The harvest of marine fish provides 20 percent of the animal protein consumed worldwide by roughly 3 billion people with an annual wild capture that averages about 90 million metric tons (FAO, 2012). Motivated by high demand and faced with the progressive depletion of coastal fisheries, commercial fishing operations have probed deep offshore waters in search of new, exploitable fish stocks (Morato et al., 2006; Garcia et al., 2008; Simpfendorfer and Kyne, 2009). Deep-sea chondrichthyan fauna are poorly understood, and little to nothing is known about the influence that bycatch attrition associated with commercial fishing has on their populations (Simpfendorfer and Kyne, 2009).

Concurrent with recent fisheries expansion, new chondrichthyan species are being discovered and described at a rate that exceeds any previous period of time since the advent of the Linnaean binomial nomenclature in the mid-18 ${ }^{\text {th }}$ century (White and Last, 2012). However, although more than 200 species have been described in the last decade, there are an estimated 70 to 100 recently discovered species still awaiting formal description (Ebert et al., 2013). This figure illustrates the deficiencies in our taxonomic knowledge and documentation of chondrichthyans as a whole, especially those inhabiting the deep-sea (Last, 2007; Ebert et al., 2013). As a result, our understanding of the life histories of deep-sea chondrichthyans is often exacerbated by taxonomic confusion, complexity, and misidentification of species. 
Studies on deep-sea chondrichthyans until recently have been inhibited by technological difficulties associated with sampling at great depths. However, technological advancements have enabled the expansion of deep-sea fisheries to probe this previously little explored environment (Haedrich et al., 2001). Also critical to an understanding of deepwater chondrichthyans has been the collaboration between commercial deep-sea fishing corporations and chondrichthyan researchers. Prior to this development, deepwater sharks and rays landed as bycatch were rarely retained and were often misidentified, thereby providing limited or incorrect information on these species and the influence of the fisheries on their populations (Kyne and Simpfendorfer, 2010). Unfortunately, misidentification is a common challenge associated with demographic and population assessments of deep-sea chondrichthyans, and taxonomic uncertainties, along with undescribed species are contributing factors in limiting our knowledge of these poorly known deep-sea sharks and rays (White and Last, 2012).

Effective and sustainable ecosystem-based management requires accurate identification of regional species. As the foundation upon which biological sciences rest, accurate and universal taxonomy is essential to this management goal (Simpfendorfer et al., 2011). The history of marine science, however, has seen examples of misdirected management efforts based on unclear taxonomy. Thunnus albacares (Yellowfin Tuna), a single species with global distribution, was originally known by 27 different identities around the world (Gibbs and Collette 1967). Conversely, Scomberomorus (the Spanish mackerel), actually two species, S. maculatus (Atlantic Spanish mackerel) and $S$. 
brasiliensis (Serra Spanish mackerel) with very different sizes of maturity, was for many years classified as a single species (Collette et al., 1978).

Sharks and rays are no exception to the dilemma of taxonomic uncertainty. There have been similar cases where a single chondrichthyan species has been represented by different names, e.g., Centrophorus granulosus (Gulper Shark) (White et al., 2013) and Etmopterus granulosus (Southern Lantern Shark) (Straube et al., 2015), and where multiple species have been thought to be a single species, as exemplified by the critically endangered Dipturus batis (Common Skate ) (Griffiths et al., 2010). This once prolific skate was fished to near extinction and was listed as critically endangered by the IUCN (Dulvy et al., 2006). The Common Skate has since been shown to be composed of two distinct species with different sizes at maturation. Therefore, the population size of each individual species is smaller than previously estimated and is likely more endangered than assessed together as a complex (Iglesias et al., 2010). This example highlights the importance of accurate taxonomy and valid life-history studies. Cases such as this confound management efforts and call into question earlier life-history studies (Griffiths et al., 2010; Iglesias et al., 2010).

The Squalus acanthias (Spiny Dogfish) species complex is another example of taxonomic confusion. Once considered underutilized, S. acanthias is currently assessed as Vulnerable globally by the IUCN due to pressure from overfishing. A widespread species, S. acanthias has populations in the Atlantic, Indian, and Pacific Oceans. After the European stocks decreased due to overexploitation, S. acanthias was commercially targeted off the east and west coasts of North America (White and Last, 2012). However, 
North Pacific and western Atlantic populations previously thought to be a single species have recently been shown to represent two distinct species: Squalus acanthias, with a global distribution in temperate regions except for the North Pacific where it is absent, and Squalus suckleyi (Pacific Spiny Dogfish), now known only to occur in the North Pacific, was recently resurrected by Ebert et al. (2010). These look-alike species, $S$. acanthias and S. suckleyi, were managed similarly even though each species has distinctly different life histories. The northwest Atlantic species (S. acanthias) matures at 12 (females) and six (males) years old compared to the North Pacific species ( $S$. suckleyi), which doesn't mature until 35.5 (females) and 18.5 (males) years (Ebert et al., 2010; Bigman et al., 2016). In addition, S. acanthias has a faster growth rate and a larger litter size of up to 25 pups compared to S. suckleyi that has a slower growth rate and a maximum litter size of 17 (Ketchen, 1972; Ebert et al., 2010). As a result, the east and west coastlines of North America require different management strategies since each species has a very different resilience to fishing pressure. This is a clear example of the importance of taxonomic understanding and its practical application in fisheries management.

The chimaeroids (Chondrichthyes: Holocephali) are among one of the most poorly known groups of cartilaginous fishes. The Chimaeriformes form a small group of cartilaginous fishes that for the most part reside in deep-sea habitats along the benthic continental shelf (Barnett et al., 2006; Didier et al., 2012). The order comprises three families, six genera, and 49 recognized species (Weigmann, 2016; Eschmeyer and Fong, 2017). The family Callorhinchidae is commonly referred to as the plow-nose chimaeras 
since a soft plow-shaped snout characterizes members of this family. This family is the least diverse of the chimaeroids with only one genus and three shallow water species (Didier et al., 2012; Nelson, 2016). The family Rhinochimaeridae is referred to as the long-nose chimaeras, with members identifiable by their very elongated snout. This family has three genera: Harriotta (two species), Neoharriotta (three species), and Rhinochimaera (three species) (Didier et al., 2012; Nelson, 2016). The family Chimaeridae is referred to as the short-nose chimaeras, with its members characterized by a short, blunt, conical snout. This family Chimaeridae is globally distributed with the exception of polar waters (Ebert and Winton, 2010), and displays a high degree of endemism (Didier et al., 2012). It is the most speciose family of chimaeroids, represented by two genera, Chimaera, and Hydrolagus, with 14 and 23 species, respectively (Didier et al., 2012; Angulo et al., 2014; Eschmeyer and Fong, 2014). The number of species has increased by 20 since 2002 and is likely to increase further with several undescribed species known to exist, but awaiting formal descriptions (Kemper et al., 2015; Eschmeyer and Fong, 2017; Walovich et al., 2017).

Despite the fact that commercial fisheries are active in the Southwestern Indian Ocean (SWIO), very little research exists addressing the deep-sea chondrichthyans from this large ecosystem. In order to predict the vulnerability of deep-sea chondrichthyans to the overharvesting and/or bycatch attrition associated with commercial exploitation of deep-water resources, researchers must know the maturation and growth characteristics of these fauna. Species-specific life-history information and taxonomic clarity are needed 
to generate models with which to monitor deep-sea shark populations within deep-sea fisheries.

The goal of this study is to catalogue the chondrichthyans fauna of the SWIO along the Madagascar Ridge and Southwest Indian Ocean Ridge, and to build a baseline of life history and population data. Chapter 1 is a taxonomic study describing three species of chimaeroids that appear to be morphologically distinct from any currently known species. Chapter 2 catalogues all the chondrichthyan species encountered from two field seasons (totalling 113 days) on board a deep-sea commercial trawler in the SWIO, and compiles an overview of associated life history information collected, e.g. general biology, length data, maturity, reproduction, distribution, and diet. Additionally, this chapter examines species abundance and species richness between the major ecosystems studied within the SWIO.

The broader objective of this project aims to provide qualitative and quantitative descriptions on the life history characteristics of deep-sea sharks to inform policy makers. Informative data is required for improved development of ecosystem-based management strategies for the conservation of deep-sea chondrichthyan fauna. Such strategies could guide policy decisions that promote sustainable fisheries and conserve deep-sea ecosystems. 


\section{General Materials and Methods}

\section{Survey Region}

Submarine Topology. High-relief mountains and canyons characterize the topography of the SWIO sea floor (Clark, 2009). One of the more prominent features, the Madagascar Ridge, extends $1300 \mathrm{~km}$ south of Madagascar as a massive plateau dividing the largest ecosystems in the region — the Southwest Indian Ocean Offshore-into two deep ocean basins (Goslin et al., 1980; Sinha et al., 1981; Collette and Parin, 1991). To the west of the Madagascar Ridge is the Mozambique Basin and to the east is the Madagascar Basin (Figure 1); both basins descend to a depth of $>5,000 \mathrm{~m}$. By comparison, the Madagascar Ridge is relatively shallow (1,500 to 2,000 m depth) (Goslin et al., 1980; Sinha et al., 1981; Collette and Parin, 1991).

The Madagascar Ridge is punctuated by a series of seamounts (Goslin, 1980). These steeply sloped topographical features function as isolated underwater islands, supporting high abundance, diversity, and endemism of fish species (Clark, 2009). The shallowest of these seamounts, Walters Shoal, is located on the southwest part of the Madagascar

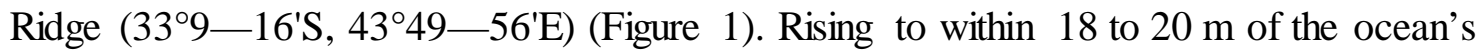
surface, this isolated cluster of submerged mountains serves as habitat for a diverse and unique composition of species, including a diverse group of apex predators, such as sharks (Hearn et al., 2010). 


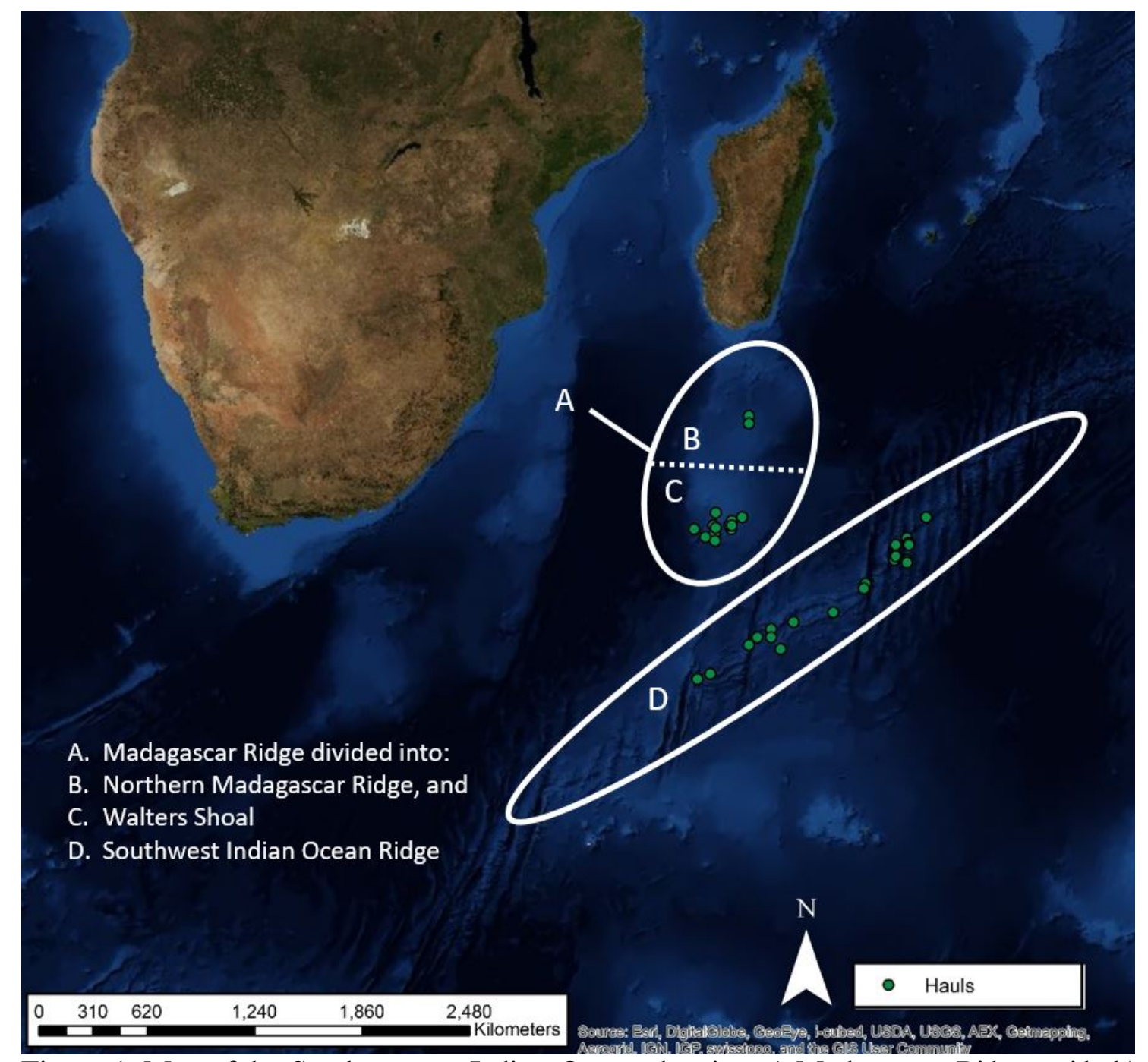

Figure 1. Map of the Southwestern Indian Ocean showing a) Madagascar Ridge, with b) the northern region, c) southern region (Walters Shoal), and d) the Southwest Indian Ocean Ridge. Trawl locations indicated by green circles; 1 March to 23 April 2012 and 10 April to 7 June 2014. Map data: Google, Image (C) 2017 DigitalGlobe.

Previous Exploration. Initial research efforts in the offshore area of the SWIO occurred around Walters Shoal in 1964 when the U.S. R/V Anton Bruun collected bottom samples via rock dredge that resulted in the description of seven species of invertebrates (Clark, 1972; Kensley, 1975). However, before the establishment of the 200-mile Exclusive Economic Zones (EEZ) by several Indian Ocean coastal countries in the mid- 
1970s, little was known about the offshore fauna of the SWIO. With the restrictions imposed by EEZs, several countries, notably, the Union of the Soviet Socialist Republic (USSR) began prospecting for exploitable fisheries resources in the deep-sea of the Southwest Indian Ocean Offshore ecosystem (Romanov, 2003).

Between 1973 and 1978, USSR exploratory cruises worked at Walters Shoal and provided some information on fish species of the area including data from a large collection of sharks (Gubanov, 1985; Gubanov, 1988; Collette and Parin, 1991). In 1976, the French trawler, Cape-Horn, collected sharks, rockcod, and lobsters from the Walters Shoal area (Collette and Parin, 1991). Since the mid-1980s, there has been little research activity in the SWIO offshore region.

\section{Field Sampling}

Study Site and Field Seasons. A suite of natural and life history data (species, sex, length, maturity, reproduction, location, depth, and diet) from captured chondrichthyans were collected from 1 March to 23 April 2012 during a 54-day expedition, and again from 10 April to 7 June 2014 during a 59-day trip aboard the New Zealand-based deepsea commercial trawler F/V Will Watch. The survey area extended over the Southwest Indian Ocean Ridge and the Madagascar Ridge in a remote region of the SWIO described by $29^{\circ} 34^{\prime}-40^{\circ} 40^{\prime} S ; 43^{\circ} 10^{\prime}-55^{\circ} 15^{\prime} \mathrm{E}$ (Figure 1). All animals involved in this study were sampled in accordance with Institutional Animal Care and Use Committees (IACUC) protocol \#801 and 2014-D.

Specimen Collection. Otter trawl nets were deployed at 40 stations and towed downward along the slopes of seamounts at an initial minimum depth of $200 \mathrm{~m}$ to a final 
maximum depth of 1,400 m. Roller-equipped bottom trawls with a $15 \mathrm{~m}$ mouth width, 50 $\mathrm{m}$ wing span, and $5 \mathrm{~m}$ height were towed forward while making contact with the slopes of the seamounts. Mid-water trawls were towed approximately $2 \mathrm{~m}$ off slope surfaces, and utilized an 80-100 m wingspan and a $35 \mathrm{~m}$ height.

Following net retrieval, bycatch was sorted and processed in the ship's factory. A census of species and sex data was taken to ensure integrity of species distribution and sex ratios. A total of 4009 individual sharks including 31 species from 14 genera were identified, sorted, counted, and photographed, and a suite of biological parameters were recorded. A list of species encountered is included in Table 1 (in Chapter two).

Voucher specimens were shipped to Moss Landing Marine Laboratories for further study. Post-preservation morphometric data were taken point-to-point to the nearest millimeter using a slide caliper and measuring tape modified from Didier and Séret (2002) for chimaeroid specimens, and depending on the shark taxa, modified from Compagno (2001) with the following references to provide taxa specific details for the groups Centrophorus (White et al., 2013) Etmopterus (Ebert et al., 2011) and for Apristurus (Nakaya et al., 2008), and Bythaelurus (McCosker et al., 2012). Specimens were deposited into museum collections at the California Academy of Sciences (CAS), Museum of Comparative Zoology (MCZ), National Museum of Natural History, Smithsonian (USNM), Natural History Museum (BMNH), Scripps Institution of Oceanography, Marine Vertebrate Collection (SIO), South African Institute for Aquatic Biodiversity (SAIAB), and Iziko-South African Museum (iSAM MB). Comparative material was examined from the following institutions: American Museum of Natural 
History (AMNH), Academy of Natural Science of Philadelphia (ANSP), CAS, Commonwealth Scientific \& Industrial Research Organization, Division of Marine \& Atmospheric Research, Hobart, Tasmania (CSIRO), Field Museum of Natural History, Zoology Department, Chicago, Illino is (FMNH), Hokkaido University Museum, Fisheries Science Center, Hakodate, Hokkaido (HUMZ), iSAM MB, MCZ, Natural History Museum of Los Angeles County, Los Angeles, California (LACM), Museo Nacional de Historia Natural, Montevideo (MNHN), SAIAB, SIO, and USNM. Institutional accession numbers will be assigned to all specimens deposited in ichthyology collections. Institutional acronyms follow Sabaj (2016). 


\section{Chapter One: New species of Chimaera (Chondrichthyes: Holocephali: Chimaeniformes: Chimaeridae) from the Southwestem Indian Ocean}

\section{Introduction}

The Chimaeriformes (Chondrichthyes: Holocephali) are a small group of cartilaginous fishes that mostly reside in deep-sea benthic habitats along continental shelves and seamounts (Didier et al., 2012). The order comprises three families, Callorhinchidae Garman, 1901 (plow-nose chimaeras), Rhinochimaeridae Garman, 1901 (long-nose chimaeras), and Chimaeridae Bonaparte, 1831 (short-nose chimaeras), with the latter family being the most species-rich with two genera and 39 described species (Didier et al., 2012; Kemper et al., 2015; Weigmann, 2016; Eschmeyer et al., 2017; Walovich et al., 2017). The two genera, Chimaera Linnaeus 1758 and Hydrolagus Gill 1862, are morphologically very similar, both being characterized by a short, blunt, fleshy snout and an elongated body that tapers to a filamentous, whip-like tail (Kemper et al., 2010a; Didier et al., 2012). The genera can be distinguished by the presence (Chimaera) or absence (Hydrolagus) of an anal fin (Gill, 1862). The genus Chimaera currently has 16 valid species, with possibly four or more undescribed species, while Hydrolagus has 24 valid species with five or six undescribed species (Kemper et al., 2015; Walovich et al., 2017; D.A. Ebert, pers. database). Since 2002, 10 new Chimaera species, and 10 new Hydrolagus species have been described (Didier et al., 2012; Angulo et al., 2014; Kemper et al., 2015; Weigmann, 2016; Eschmeyer et al., 2017; Walovich et al., 2017).

The Chimaeridae has a global distribution, occurring in most seas except for polar waters (Ebert and Winton, 2010). Because many species are deep-sea inhabitants in remote regions, the family is difficult to sample and consequently, remains poorly 
understood (Didier et al., 2012). For example, the taxonomic status of Western Indian Ocean chimaeroid species is unsettled, with only the poorly known Hydrolagus africanus (Gilchrist, 1922) having been confirmed from this region (Ebert, 2014). Hydrolagus africanus was originally described from a specimen collected off Durban, South Africa, but has been reported as occurring from Angola to Kenya, and possibly from India (Compagno et al., 1989; Walovich et al., 2015). Hydrolagus africanus has also been reported from off seamounts in the Southwestern Indian Ocean (SWIO), but without confirmation (Novikov, 2002), while Chimaera notafricana Kemper, Ebert, Compagno, \& Didier 2010 was described from southern Africa, and has a restricted distribution from Algoa Bay, Eastern Cape Province, South Africa to Lüderitz, Namibia (Kemper et al., 2010a; Ebert, 2014).

Two surveys were recently conducted (2012 and 2014) in the SWIO, in the same general area where Novikov (2002) reported finding H. africanus, but no specimens of that species were collected or observed. However, three relatively large Chimaera species were collected from very deep water and retained. Upon closer examination, it was determined that all three species were distinct from all other known members of the family. Here we describe these three new species from the SWIO (currently in press). This paper is part of a series describing new species and revising chondrichthyan taxa from the SWIO.

\section{Materials and Methods}

Study Location. The survey area extended over a remote region of the SWIO encompassing an area described approximately by $33^{\circ} 50^{\prime}$ to $40^{\circ} 40^{\prime} \mathrm{S}, 43^{\circ} 10^{\prime}$ to $55^{\circ} 15^{\prime} \mathrm{E}$ 
(Figure 1). The SWIO sea floor topography is characterize by mountains of high relief and submerged canyons (Clark, 2009). One of the more prominent of these features is the Madagascar Ridge that extends $1300 \mathrm{~km}$ south of Madagascar as a massive plateau dividing the largest ecosystems in the region, the Southwest Indian Ocean Offshore (Goslin et al., 1980; Sinha et al., 1981; Collette and Parin, 1991). The northern half of the Madagascar Ridge is relatively shallow (1,500 to 2,000 m depth) and characterized by steeply sloped topographical and complex habitat (Goslin, 1980; Clark, 2009). To the south is a series of shallow, flat-topped seamounts. The shallowest of these seamounts, Walters Shoal, rises to within 18 to $20 \mathrm{~m}$ of the sea surface. To the southeast, the Southwest Indian Ocean Ridge bisects the ocean between Africa and Antarctica. Rifted crests, and rugged mountainous flanks characterize this enormous, and continuous, ridge, which supports a diverse and unique composition of species (Hearn et al., 2010). These three distinct ecosystems were surveyed during two expeditions, the first from 1 March to 23 April 2012 (54 days total) and a second from 10 April to 7 June 2014 (59 days total), aboard the New Zealand-based deep-sea commercial trawler F/V Will Watch.

Specimen Collection. Specimens were collected as bycatch, identified, measured, and sexed. Specimens of each species were photographed fresh, tissue samples were removed and stored in $100 \%$ ethanol for later genetic studies, frozen for the duration of the cruise, and retained for further study. Upon returning to port all specimens retained were initially preserved in $10 \%$ buffered formalin and later transferred to $70 \%$ ethanol for storage.

Measurements/Meristics. Morphometric measurements have traditionally been the standard parameter for separating species based on measureable factors between 
consistently identifiable homologous points in order to compare geometric form differences (Bookstein et al., 1985). Morphometric measurements, while low tech, are compatible with the scientific records predating the use of genetic information and, unlike more sophisticated methods, are immediately applicable for use in the field. Detailed morphometric measurements were taken on preserved specimens and normalized as a ratio of body length, and are expressed as a proportion for comparison. Body measurements and lateral line canal measurements were taken point-to-point to the nearest millimeter (mm) following Didier and Séret (2002), and were modified with the addition of 53 new measurements (Figures 2-5). These new measurements were added to quantify previously qualitative characteristics (e.g. rate of tapering from body to tail, blockiness of head, breadth of fins). In total 91 (69 body, 11 clasper and tenaculum, 3 post anal pad, and 8 lateral line canal) measurements were recorded (see Appendix A for definition and explanation of abbreviations).

Two of the new species are described from a single specimen, but the third Chimaera species had a total of 57 specimens (38 females, 19 males) that were collected. Based on these 57 specimens with a body length (BDL) range of $118-645 \mathrm{~mm}$, and using the morphological measurements referred to above, we examined possible ontogenetic changes associated with growth in this new species. A linear regression was used to study ontogenetic shifts with the null hypothesis that measurements (as a ratio of BDL) were consistent over BDL. Measurements with a slope that varied significantly from zero (pvalue $<0.05$ ) were considered to vary significantly with growth and ontogeny. 


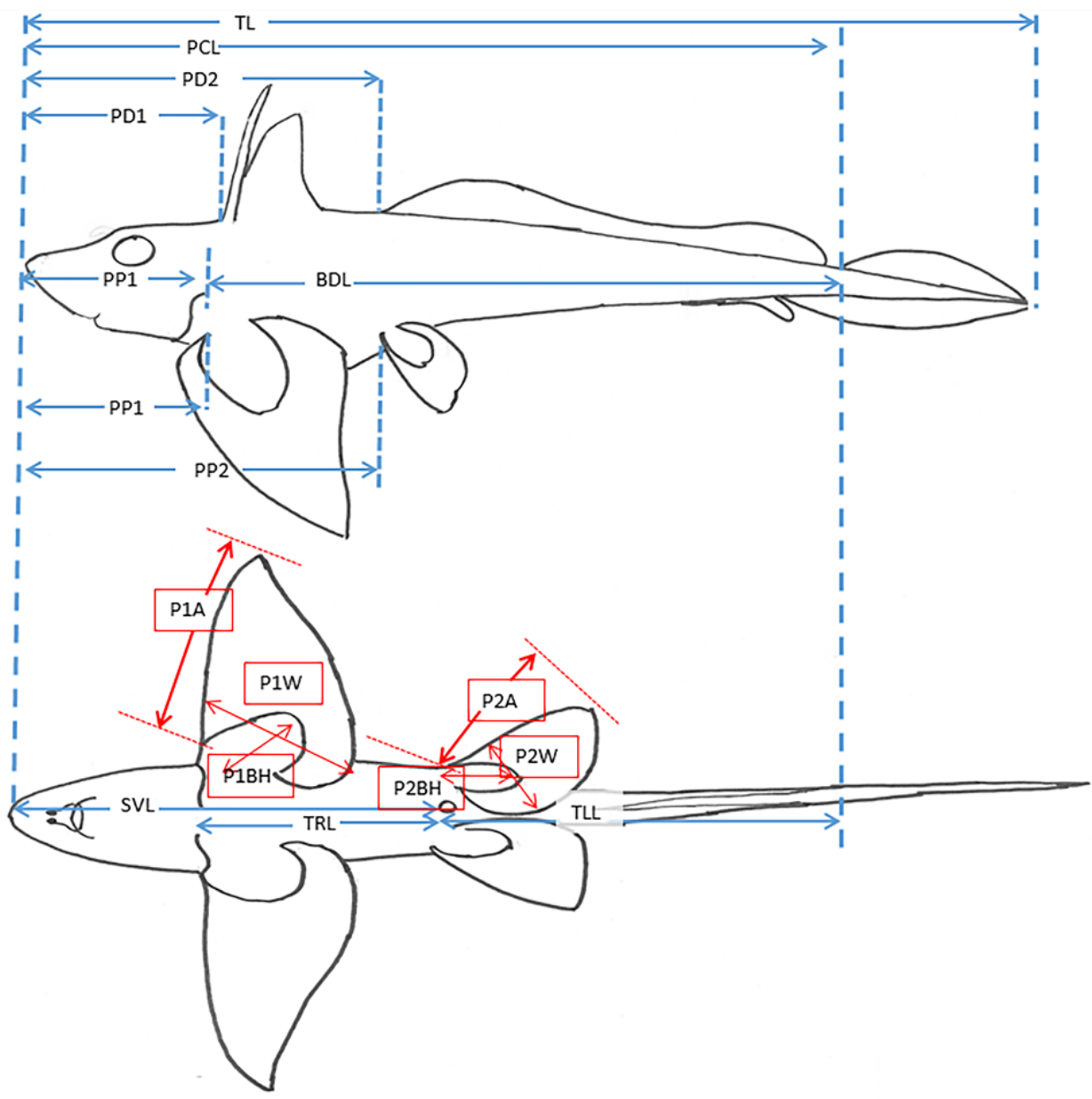

Figure 2. Diagram of lengths (blue) and paired fin measurements (red). Illustration by P.J. Clerkin. See Appendix A for definition and explanation of abbreviations. 


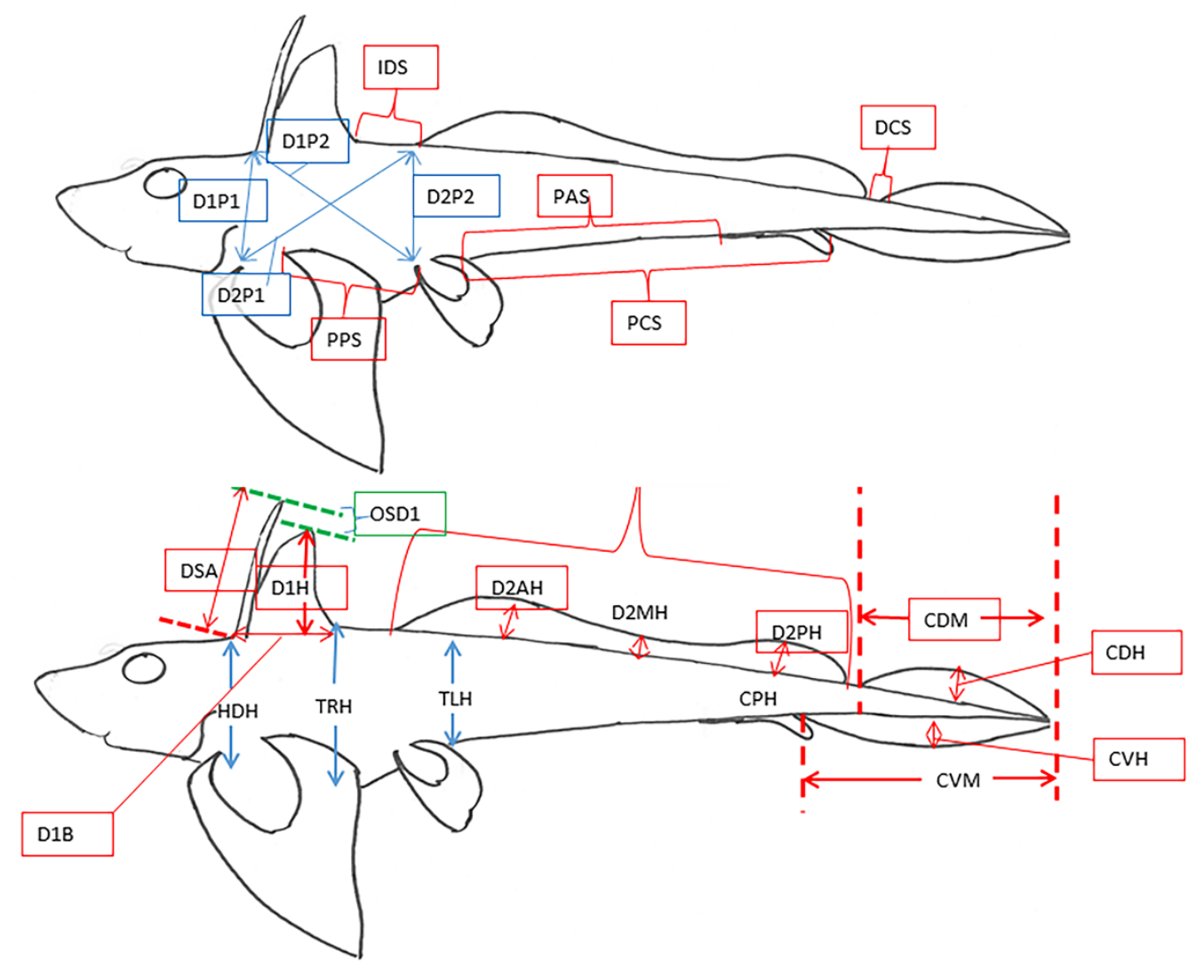

Figure 3. Diagram of measurements: a) across body (blue), inter fin spaces (red), b) body heights (blue), unpaired fin heights and margins (red), and overlap (green). Illustration by P.J. Clerkin. See Appendix A for definition and explanation of abbreviations. 


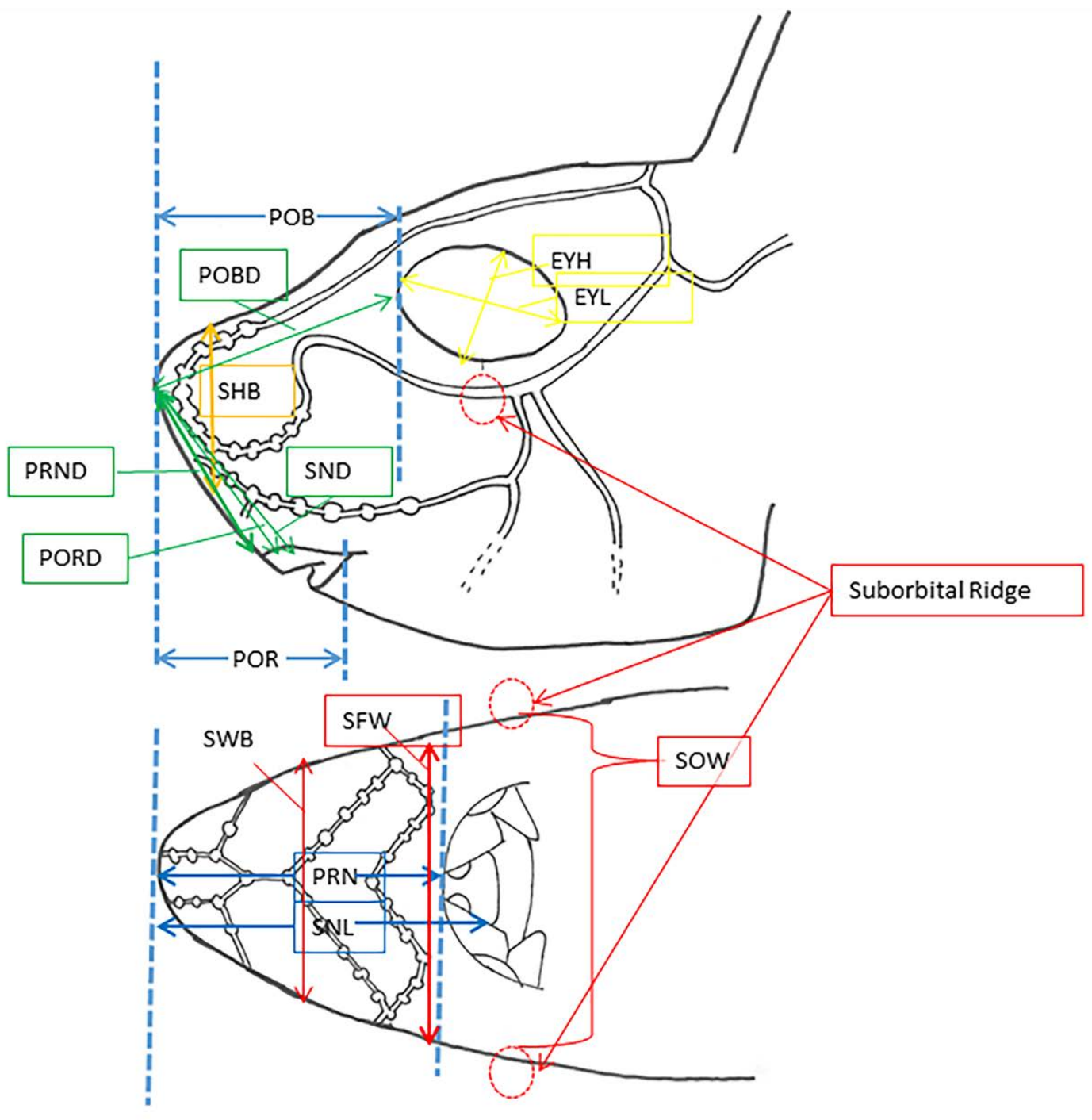

Figure 4. Diagram of head measurements: lengths (blue), widths (red), direct distance (green), eyes (yellow), height (purple). Illustration by P.J. Clerkin. See Appendix A for definition and explanation of abbreviations. 


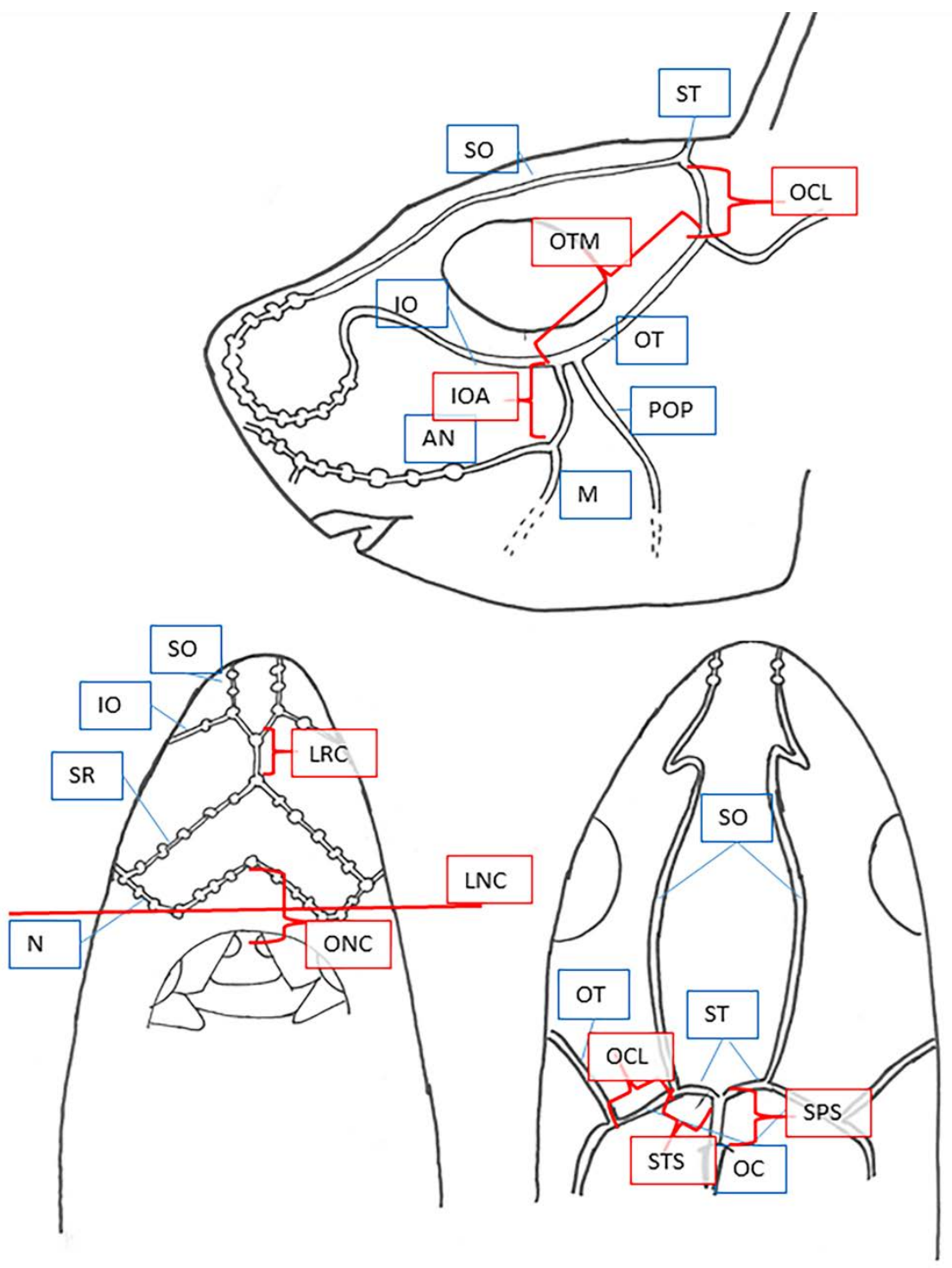

Figure 5. Diagram of canals of the head (blue) and canal measurements (red). Illustration by P.J. Clerkin. See Appendix A for definition and explanation of abbreviations.

Cluster Analysis. Ordination analysis was run in PRIMER to cluster related morphometric features of the holotype, paratypes (both large and small size classes), nontypes, and comparative material (comprised of 98 specimens, 10 species, two genera, and 29 parameters) to test for significant differences between species and their congeners. Non-metric multidimensional scaling (nMDS) scores were plotted to illustrate 
morphological dissimilarity between the chimaeroid species of this study. SIMPER pairwise comparisons of morphometric measurements were used to quantify the top five traits defining Chimaera willwatchi (the only species description with multiple type specimens) from each species compared. Additionally, the overall 15 most defining characters were calculated for diagnostic purposes.

Genetic Analysis. The last two decades has seen an explosion of molecular-based studies, which, by using molecular markers in combination with morphometric measurements can be a useful tool to distinguish species (White and Last, 2012; Kemper et al., 2015). Total DNA was extracted from muscle tissue using the EZNA ${ }^{\circledR}$ Tissue DNA Kit (Omega Bio-Tek) and stored at $-20^{\circ} \mathrm{C}$. The complete coding sequence for the mitochondrial NADH dehydrogenase subunit 2 (NADH2) gene was PCR amplified and sequenced according to Kemper et al. (2015). The program Geneious (version 6.1.7) was used to read chromatograms, view and correct nucleotide base calls, and align nucleotide and translated sequences. No sequences contain missing data. The complete dataset included 53 nucleotide sequences from ten chimaeroid species, with an alignment length of 1044 base pairs. Hydrolagus africanus was chosen as an outgroup taxon. RAxML v8.1.22 (Stamatakis 2014) was used to perform a maximum likelihood search (1,000 runs on distinct starting trees) using the rapid hill-climbing algorithm on the aligned nucleotide sequences under the general-time reversible (GTR) nucleotide substitution model and gamma distribution for among-site rate heterogeneity, with subsequent nonparametric bootstrapping of 1,000 replicates. NADH2 sequences were deposited in GenBank (see Appendix B). 
Chimaeroid species identification is very challenging because of morphological and color similarities between species and variation within species (Kemper et al., 2015). This study uses both morphological and molecular techniques to evaluate and describe new species encountered during the surveys.

Institutional Acronyms. Institutional acronyms follow Sabaj (2016). Type specimens were deposited into the California Academy of Sciences (CAS), Museum of Comparative Zoology (MCZ), National Museum of Natural History, Smithsonian (USNM), Natural History Museum (BMNH), Scripps Institution of Oceanography, Marine Vertebrate Collection (SIO), South African Institute for Aquatic Biodiversity (SAIAB), and IzikoSouth African Museum (iSAM MB). Comparative material was examined from the following institutions: American Museum of Natural History (AMNH), Academy of Natural Science of Philadelphia (ANSP), CAS, Commonwealth Scientific \& Industrial Research Organization, Division of Marine \& Atmospheric Research, Hobart, Tasmania (CSIRO), Field Museum of Natural History, Zoology Department, Chicago, Illinois (FMNH), The Hokkaido University Museum, Fisheries Science Center, Hakodate, Hokkaido (HUMZ), iSAM MB, MCZ, Natural History Museum of Los Angeles County, Los Angeles, California (LACM), Museo Nacional de Historia Natural, Montevideo (MNHN), SAIAB, SIO, and USNM.

\section{Chimaera willwatchi, sp. nov., Seafarer's Ghost Shark}

Holotype. CAS 242336, 834+ mm TL, 492mm BDL, mature male, Southwestern

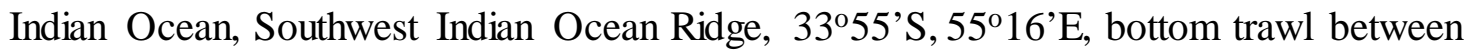
850 m-1075 m, collected by P.J. Clerkin, 24 May 2014. 
Paratype. -9 male, 9 female specimens - CAS 242337, mature male, 823+ mm TL, 456 mm BDL, Southwestern Indian Ocean, Southwest Indian Ocean Ridge, 3356’S, 55¹’’E, midwater trawl between 1008 m-1190, collected by P.J. Clerkin, 28 April 2014; CAS 242339, immature male, 843+ mm TL, 466 mm BDL, Southwestern Indian Ocean, Southwest Indian Ocean Ridge, 3508’S, 55¹7’E, bottom trawl between 89 m-1240 m, collected by P.J. Clerkin, 30 April 2014; CAS 242354, mature male, 694+ mm TL, 369 mm BDL, Southwestern Indian Ocean, Walters Shoal, 2951'S, 4603’E, bottom trawl between 1003 m-1200 m, collected by P.J. Clerkin, 31 April 2014; USNM 440273, immature male, 667+ mm TL, 461 mm BDL, Southwestern Indian Ocean, Southwest

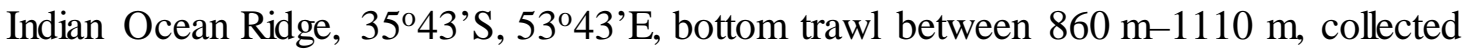
by P.J. Clerkin, 23 April 2014; MCZ 171972, mature male, 782+ mm TL, 490 mm BDL,

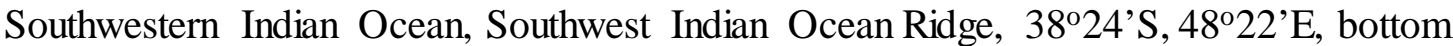
trawl between 680 m-970 m, collected by P.J. Clerkin, 18 March 2012; SIO 16-67, mature male, 739+ mm TL, 403 mm BDL, Southwestern Indian Ocean, Southwest Indian

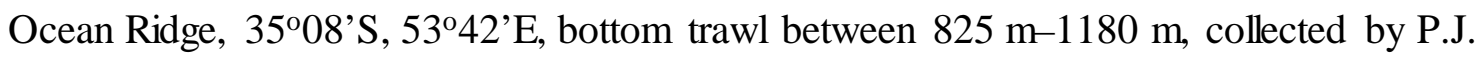
Clerkin, 17 March 2014; CAS 242338, mature female, 913+ mm TL, 587 mm BDL,

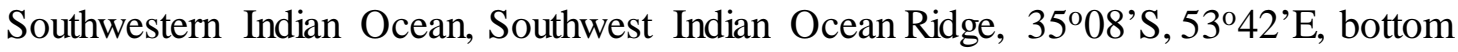
trawl between 874 m-1118 m, collected by P.J. Clerkin, 26 March 2014; CAS 242337, female, 804+ mm TL, 525 mm BDL, Southwestern Indian Ocean, Southwest Indian

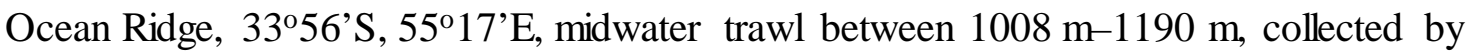
P. J. Clerkin, 28 April 2014; CAS 242343, female, 770+ mm TL, 455 mm BDL, Southwestern Indian Ocean, Southwest Indian Ocean Ridge, 3902’S, 46³3’E, bottom 
trawl between 777 m-1178 m, collected by P. J. Clerkin, 17 March 2012; CAS 242367, female, 920+ mm TL, 522 mm BDL, Southwestern Indian Ocean, Walters Shoal,

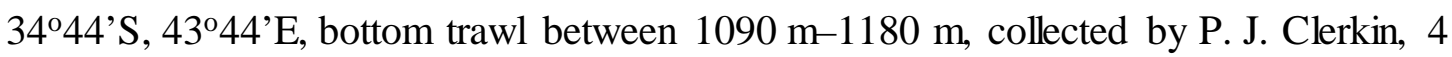
April 2014; USNM 440274, mature female, 948+ mm TL, 604 mm BDL, Southwestern

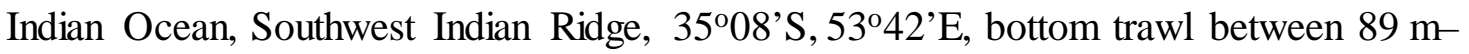
1240 m, collected by P.J. Clerkin, 30 April 2014; SAIAB 203575, mature female, 826+ mm TL, 576 mm BDL, Southwestern Indian Ocean, Southwest Indian Ocean Ridge, 3509’S, 5343'E, bottom trawl between 880 m-1200 m, collected by P.J. Clerkin, 23 April 2014; SIO 16-68, mature female, 821+ mm TL, 519 mm BDL, Southwestern Indian

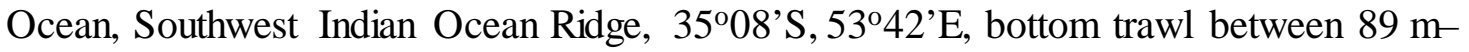
1240 m, collected by P.J. Clerkin, 30 April 2014; MB-F035527, immature male, 661+ mm TL, 397 mm BDL, Southwestern Indian Ocean, Southwest Indian Ocean Ridge, 38²2’ S, 47³5’ E, bottom trawl between 700 m-960 m, collected by B. Walkins, 17 January 2000; MB-F035739, female, 845+ mm TL, 478 mm BDL, Southwestern Indian Ocean, Prince Edward Islands, 39 50' S, 45 47' E, trawl between 700 m-982 m, collected by B. Walkins, 3 May 2001; MB-F035739, mature male, 546+ mm TL, 519 mm BDL, Southwestern Indian Ocean, Prince Edward Islands, 39 50' S, 45 47' E, trawl between 700 m-982 m, collected by B. Walkins, 3 May 2001; MB-F035814, female, 872+ mm TL, 545 mm BDL, Southwestern Indian Ocean, Prince Edward Islands, 39 26' S, 412 20' E, trawl between 700 m-890 m, collected by B. Walkins, 6 May 2001; MBF035815, immature male, 730+ mm TL, 399 mm BDL, Southwestern Indian Ocean, 
Prince Edward Islands, 39 26' S, 41 19' E , trawl between 700 m-888 m, collected by B. Walkins, 5 May 2001.

Non-type. - 9 male, 29 female specimens - CAS 242355, 242340, 242342, 242344, 242345, 242346, 242347, 242348, 242349, 242350, 242351, 242352, 242353, 242356, 242357, 242358. Males ranged from 479+ to $810+$ mm TL, 450 to $152 \mathrm{~mm}$ BDL,

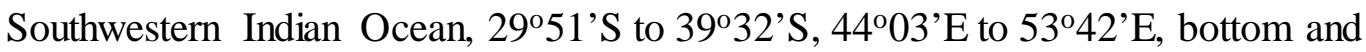
midwater trawl between 715 m-1328 m, collected by P.J. Clerkin from 6 March to 3 April 2012 and 23 April to 31 May 2014, and R. Downie between 23 March to 2 April 2014. Females ranged from 290+ to $971+\mathrm{mm}$ TL, 118 to $645 \mathrm{~mm}$ BDL, Southwestern Indian Ocean, $29^{\circ} 51^{\prime} \mathrm{S}$ to $39^{\circ} 02^{\prime} \mathrm{S}, 4^{\circ} 03^{\prime} \mathrm{E}$ to $55^{\circ} 16^{\prime} \mathrm{E}$, bottom and midwater trawl between 752 m-1340 m, collected by P.J. Clerkin from 5 March to 13 April 2012 and 18 April to 31 May 2014, and R. Downie between 23 March to 2 April 2014.

Diagnosis. Chimaera willwatchi, sp. nov. is a large species at maturity (971 mm TL, $645 \mathrm{~mm}$ BDL) distinguished from all other chimaeroids by the following combination of characters: head blocky, large followed by stocky trunk, body height fairly constant from trunk (pectoral fin origin) to abdomen (pelvic fin origin) before tapering rapidly into long tail; large eyes, and well-defined, blocky suborbital ridge; blunt, distinctly squared snout (Table 1). Paired claspers externally trifurcate, forked distal one-third of length, prepelvic tenacula each with 4 large, tooth-like denticles tightly spaced along medial edge. Brownish skin with iridescent wash; brown and white marbled marking around snout, mouth, and ventral half of trunk; posterior margin of first dorsal fin very distinctly white, with white distal margins on anterior half of second dorsal fin, and posterior margins of 
pelvic fins; dorsal spine, exceeds the apex of the first dorsal fin and, when depressed, slightly overlaps the origin of the second dorsal fin, large second dorsal fin not obviously undulating; caudal fin very large and paddle-shaped. Structure of the NADH2 gene.

Chimaera willwatchi, sp. nov. can be distinguished from its closest congeners, Chimaera lignaria Didier 2002, Chimaera macrospina Didier et al. 2008, and Chimaera orientalis Angulo et al. 2014, by a combination of characters: large dorsal spine exceeding apex of first dorsal fin, long, trifurcated claspers, prepelvic tenacula with 4 spines, robust body, large caudal fin, large pelvic fin anterior margin, and coloration.

Table 1. SIMPER analysis of morphometric measurements defining Chimaera willwatchi. Displayed are the top fifteen ranked morphometric measurements (all with contributing percentages above 3.00) which distinguish Chimaera willwatchi as a species. The contributing percentages are expressed as \%.

\begin{tabular}{|l|c|}
\hline Morphometric Measurement & Contributing \% \\
\hline \hline Tail length & 7.96 \\
\hline Trunk length & 6.48 \\
\hline Pectoral fin anterior margin & 6.06 \\
\hline Max Trunk height & 5.30 \\
\hline Head height & 5.05 \\
\hline Head length & 5.05 \\
\hline Pelvic anterior margin & 4.88 \\
\hline Pelvic anterior margin & 4.68 \\
\hline Pectoral fin width & 3.88 \\
\hline Head width at suborbital ridge & 3.81 \\
\hline Tail height & 3.76 \\
\hline Pelvic fin width & 3.68 \\
\hline Trunk width & 3.15 \\
\hline Abdominal Width & 3.12 \\
\hline preopercular to main trunk & 3.10 \\
\hline
\end{tabular}


Description. Morphometric proportions for the holotype, with ranges for large $(\geq 519$ mm BDL) paratypes (male and female separate), and ranges of small non-types $(<300$ mm BDL; male and female combine) are presented in Table 2. The following description proportions include the holotype followed by paratypes of large specimens with sexes combined in parentheses. Additional descriptive information of small specimens highlighting ontogenetic differences is also provided.

A large-bodied species reaching up to $519 \mathrm{~mm}$ BDL in males and $645 \mathrm{~mm}$ BDL in females. Head huge, blocky with prominent subocular ridges, head height $26.6 \%$ (23-26.7\%) BDL, length about one-fifth (20.6\%) precaudal length; snout short, blunt, length about one-half head length; nostrils and mouth below ventral contour of snout; prenarial length $4.0 \%(3.0-7.7 \%)$ BDL. Trunk slightly compressed, body depth similar to head height, maximum depth occurs mid trunk, height 30.3\% (22.7-30.7\%) BDL, gradually tapering to pelvic girdle, abdomen height $26.8 \%$ (18.4-24.9\%) BDL, tapering rapidly to relatively thin tail, $16.6 \%(12-24.5 \%) \mathrm{BDL}$, and continuing to a caudal peduncle height 3.2\% (2.4-3.0\%) BDL. Tail long, making up about one-half (49.5\%) precaudal length, relative to trunk length (34.3\% of the precaudal length) and head length (22.0\% precaudal length). Eyes large, rounded, length about one-third head length, 8.5\% (7.6-9.2\%) BDL, height about one-fourth head length, 6.5\% (5.1-7.0\%) BDL; preorbital length $28 \%$ head length. Interdorsal-space short to moderately long, 3.3\% (4.6-10.1\%) BDL. Pectoral-pelvic space 34.9\% (30.0\% -36.2\%) BDL, 1.2-1.4 times head length, and shorter than pelvic-caudal space. Pelvic-caudal space 52.3\% (49.3-54.5\%) BDL, about 
1.7-2.2 times head length, and shorter than snout-vent length 67.1\% (58.2-67.1\%) BDL, 2.2-2.5 times head length. Skin somewhat deciduous, smooth without denticles.
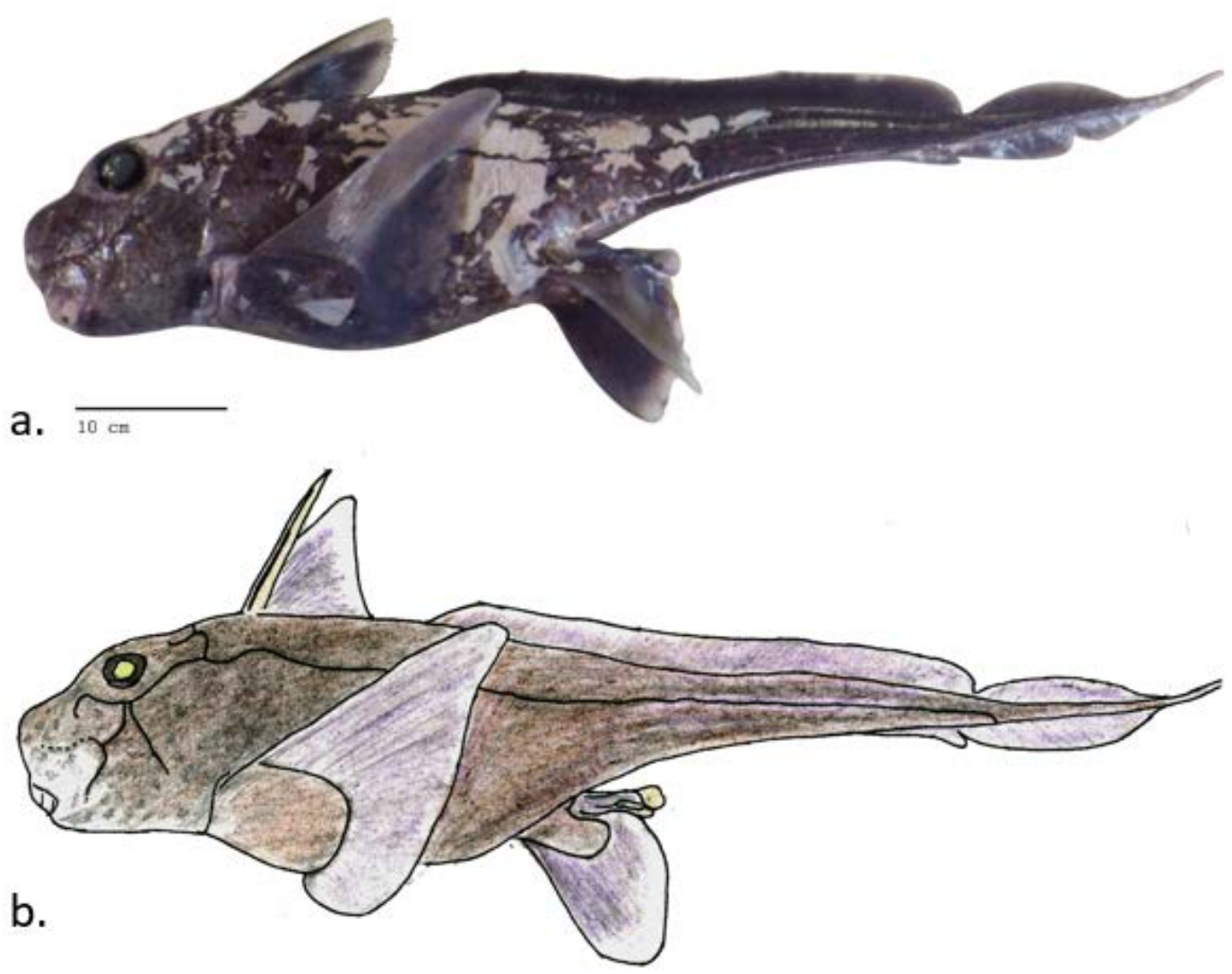

Figure 6. Full body lateral view of Chimaera willwatchi, sp. nov., holotype CAS 242336, mature male $834 \mathrm{~mm}$ TL, $492 \mathrm{~mm}$ BDL, A) Photograph, B) Illustration. Photo and illustration by P.J. Clerkin.

Pectoral fins large, broad, width $22.1 \%$ (19.6-23.2\%) BDL, anterior margin $36.8 \%$ (33.2-40.2\%) BDL, relatively straight, gradually rounding towards distal tip, posterior margin straight, inner margin rounded; when depressed posteriorly against body, pectoral fin slightly overlaps origin of pelvic fins; pectoral fin base off-round, somewhat angular in shape. Pelvic fins large, very broad, width $16.0 \%$ (11.4-16.7\%) BDL, tear-shaped, anterior margin $25.0 \%(22.9-26.8 \%)$ BDL, about two-thirds (66\%) size of pectoral fin, 
distal two-thirds of anterior margin convex, inner and posterior margins rounded with fleshy base. 
Table 2. Raw measurements (in mm) and body length proportions (\%BDL) of Chimaera willwatchi sp. nov. holotype, paratypes, and nontypes. Min = minimum; Max = maximum.

\begin{tabular}{|c|c|c|c|c|c|c|c|}
\hline \multicolumn{8}{|c|}{ Chimaera willwatchi sp. nov. } \\
\hline & \multirow{5}{*}{$\begin{array}{c}\text { Holotype } \\
\mathrm{n}=1 \\
\text { Adult } \\
\text { Male }\end{array}$} & \multicolumn{2}{|c|}{ Paratype } & \multicolumn{2}{|c|}{ Paratype } & \multicolumn{2}{|c|}{ Nontype } \\
\hline & & \multicolumn{2}{|c|}{$n=8$} & \multicolumn{2}{|c|}{$n=7$} & \multicolumn{2}{|c|}{$\mathrm{n}=40$} \\
\hline & & \multirow{2}{*}{\multicolumn{2}{|c|}{ Male }} & \multirow{2}{*}{\multicolumn{2}{|c|}{ Female }} & \multirow{2}{*}{\multicolumn{2}{|c|}{$\begin{array}{l}\text { (10 males, } 30 \\
\text { females) }\end{array}$}} \\
\hline & & & & & & & \\
\hline & & Min & Max & Min & Max & Min & Max \\
\hline Body length (mm) & 492 & 403 & 519 & 519 & 604 & 118 & 285 \\
\hline \multicolumn{8}{|l|}{ Measurement (\%BDL) } \\
\hline Total Length & 158.9 & 138.8 & 183.4 & 142.5 & 172.6 & 172.8 & 219.4 \\
\hline Pre-caudal length & 122.8 & 120.8 & 126.1 & 119.6 & 123.2 & 120.0 & 129.6 \\
\hline Snout to vent length & 67.1 & 58.2 & 67.1 & 59.0 & 63.9 & 61.9 & 71.2 \\
\hline Tail length & 57.3 & 57.3 & 64.5 & 60.8 & 62.6 & 57.9 & 61.8 \\
\hline Trunk length & 43.1 & 36.4 & 42.8 & 37.4 & 44.4 & 40.0 & 51.7 \\
\hline Head length & 27.0 & 23.6 & 28.5 & 23.0 & 28.9 & 22.3 & 29.3 \\
\hline $\begin{array}{l}\text { Pre-first dorsal fin } \\
\text { length }\end{array}$ & 30.3 & 27.9 & 31.1 & 27.9 & 31.3 & 23.8 & 36.9 \\
\hline $\begin{array}{l}\text { Pre-second dorsal fin } \\
\text { length }\end{array}$ & 46.3 & 46.9 & 51.8 & 45.9 & 53.4 & 50.5 & 59.2 \\
\hline Pre-pectoral fin length & 29.1 & 24.0 & 35.2 & 24.7 & 34.7 & 29.8 & 33.4 \\
\hline Pre-pelvic fin length & 70.9 & 60.6 & 69.1 & 62.4 & 70.0 & 64.3 & 73.0 \\
\hline Pre-orbital length & 12.7 & 11.8 & 14.0 & 12.3 & 13.1 & 11.2 & 15.5 \\
\hline Pre-orbital distance & 12.9 & 12.5 & 14.4 & 13.0 & 13.6 & 12.3 & 16.1 \\
\hline Pre-narial length & 4.0 & 4.9 & 7.7 & 3.0 & 6.3 & 4.5 & 8.3 \\
\hline Pre-narial distance & 11.1 & 9.1 & 11.8 & 7.7 & 10.7 & 9.3 & 12.2 \\
\hline Pre-oral length & 6.2 & 6.5 & 9.6 & 4.7 & 8.0 & 5.4 & 8.7 \\
\hline Pre-oral distance & 14.9 & 11.5 & 16.4 & 11.8 & 12.9 & 12.3 & 14.4 \\
\hline Snout length & 12.7 & 9.2 & 11.7 & 7.9 & 11.1 & 10.8 & 12.3 \\
\hline Eye Length & 8.5 & 7.7 & 9.2 & 7.6 & 8.7 & 10.5 & 11.7 \\
\hline Eye Height & 6.5 & 5.8 & 7.0 & 5.1 & 6.2 & 6.2 & 9.2 \\
\hline First dorsal to pectoral & 18.1 & 17.3 & 24.2 & 17.5 & 28.5 & 17.5 & 25.5 \\
\hline First dorsal to pelvic & 44.7 & 37.3 & 50.8 & 41.3 & 45.0 & 38.9 & 45.8 \\
\hline $\begin{array}{l}\text { Second dorsal to } \\
\text { pectoral }\end{array}$ & 29.2 & 27.1 & 38.5 & 26.4 & 44.6 & 33.9 & 39.7 \\
\hline Second dorsal to pelvic & 28.3 & 19.8 & 28.5 & 22.8 & 27.1 & 20.6 & 28.5 \\
\hline Snout width at base & 2.3 & 2.5 & 3.5 & 2.0 & 3.9 & 1.9 & 5.2 \\
\hline Snout anterior width & 7.7 & 6.9 & 8.6 & 6.7 & 7.9 & 5.8 & 9.6 \\
\hline
\end{tabular}




\begin{tabular}{|c|c|c|c|c|c|c|c|}
\hline $\begin{array}{l}\text { Head width at } \\
\text { suborbital ridge }\end{array}$ & 15.8 & 14.7 & 16.9 & 13.9 & 15.4 & 12.0 & 16.0 \\
\hline Trunk width & 10.0 & 12.3 & 15.0 & 9.0 & 15.8 & 11.6 & 16.2 \\
\hline Abdominal Width & 10.8 & 8.5 & 11.2 & 8.6 & 12.3 & 7.5 & 9.9 \\
\hline Tail width & 8.5 & 7.2 & 10.2 & 6.3 & 9.0 & 4.3 & 6.6 \\
\hline Caudal peduncle width & 2.3 & 1.6 & 2.2 & 1.6 & 2.0 & 1.7 & 2.0 \\
\hline Snout height at base & 8.1 & 6.9 & 9.5 & 6.8 & 8.2 & 6.9 & 10.5 \\
\hline Head height & 26.6 & 23.0 & 26.7 & 23.0 & 28.5 & 22.5 & 27.3 \\
\hline Trunk height & 30.3 & 22.7 & 30.7 & 25.6 & 28.4 & 24.1 & 31.6 \\
\hline Max Trunk height & 30.8 & 23.3 & 31.1 & 26.6 & 31.8 & 21.1 & 29.4 \\
\hline Abdomen height & 26.8 & 18.4 & 24.9 & 21.1 & 25.1 & 18.9 & 21.9 \\
\hline Tail height & 16.6 & 12.0 & 24.5 & 13.1 & 16.4 & 12.0 & 16.3 \\
\hline Caudal peduncle height & 3.2 & 2.5 & 3.0 & 2.4 & 2.8 & 2.9 & 3.7 \\
\hline Interdorsal space & 3.3 & 4.6 & 7.2 & 5.0 & 10.1 & 4.0 & 6.8 \\
\hline Dorsal-caudal space & 0.0 & 0.5 & 2.2 & 0.0 & 1.0 & 1.0 & 2.0 \\
\hline Pectoral-pelvic space & 34.9 & 30.0 & 35.9 & 33.3 & 36.2 & 34.1 & 39.3 \\
\hline Pelvic-anal space & 45.0 & 43.8 & 48.6 & 44.7 & 48.3 & 38.9 & 49.6 \\
\hline Pelvic-caudal space & 52.3 & 52.2 & 54.5 & 49.3 & 53.4 & 49.8 & 54.7 \\
\hline Pelvic-ridge space & 24.3 & 21.5 & 23.6 & 20.9 & 26.3 & 16.2 & 26.4 \\
\hline $\begin{array}{l}\text { Pectoral fin anterior } \\
\text { margin }\end{array}$ & 36.8 & 33.7 & 40.2 & 33.2 & 36.2 & 37.3 & 45.7 \\
\hline Pectoral fin width & 22.1 & 20.8 & 23.0 & 19.6 & 23.2 & 19.6 & 24.9 \\
\hline Pectoral fin base width & 12.7 & 10.5 & 12.4 & 9.5 & 12.1 & 9.5 & 12.9 \\
\hline Pectoral fin base height & 16.3 & 10.7 & 13.0 & 10.4 & 14.5 & 11.3 & 15.1 \\
\hline Pelvic anterior margin & 25.0 & 22.9 & 26.8 & 22.9 & 23.6 & 21.5 & 26.3 \\
\hline Pelvic fin width & 16.0 & 15.2 & 16.7 & 11.4 & 15.2 & 11.8 & 15.3 \\
\hline Pelvic fin base width & 7.2 & 5.1 & 7.1 & 6.0 & 7.1 & 5.2 & 7.2 \\
\hline pelvic fin base height & 8.9 & 8.5 & 9.9 & 8.2 & 10.0 & 6.7 & 9.4 \\
\hline Dorsal spine length & 27.3 & 24.8 & 24.9 & 22.9 & 24.9 & 24.3 & 26.4 \\
\hline $\begin{array}{l}\text { Dorsal spine ridge to } \\
\text { origin }\end{array}$ & 3.0 & 2.9 & 3.8 & 2.6 & 3.8 & 3.0 & 5.1 \\
\hline First dorsal fin base & 17.6 & 14.2 & 17.9 & 14.5 & 16.9 & 15.5 & 22.1 \\
\hline First dorsal fin height & 20.3 & 18 & 19.1 & 16.2 & 18.6 & 15.6 & 20.8 \\
\hline Second dorsal fin base & 74.8 & 73.2 & 77.5 & 70.1 & 75.2 & 68.8 & 74.8 \\
\hline $\begin{array}{l}\text { Second dorsal fin } \\
\text { anterior height }\end{array}$ & 6.6 & 3.8 & 7.2 & 3.9 & 6.4 & 4.9 & 7.2 \\
\hline $\begin{array}{l}\text { Second dorsal fin } \\
\text { posterior height }\end{array}$ & 5.5 & 4.1 & 6.6 & 4.7 & 6.3 & 5.2 & 6.4 \\
\hline $\begin{array}{l}\text { Second dorsal fin mid } \\
\text { height }\end{array}$ & 5.9 & 3.7 & 6.6 & 4.2 & 5.6 & 5.4 & 7.3 \\
\hline Dorsal caudal margin & 26.6 & 29.1 & 33.2 & 25.1 & 29.8 & 23.2 & 31.1 \\
\hline Dorsal caudal height & 4.1 & 3.1 & 3.7 & 2.6 & 3.5 & 2.5 & 4.5 \\
\hline
\end{tabular}




\begin{tabular}{|l|c|c|c|c|c|c|c|} 
Ventral caudal margin & 36.0 & 43.3 & 57.3 & 39.2 & 39.2 & 24.9 & 35.2 \\
\hline $\begin{array}{l}\text { Fleshy ridge to caudal } \\
\text { insertion }\end{array}$ & 64.4 & 75.2 & 91.8 & 72.2 & 72.2 & 65.7 & 79.2 \\
\hline Ventral caudal height & 4.1 & 3.2 & 4.4 & 3.2 & 4.1 & 2.5 & 5.1 \\
\hline total caudal length & 36.7 & 3.2 & 70.7 & 50.3 & 50.3 & 52.5 & 90.6 \\
\hline $\begin{array}{l}\text { Spine to second dorsal } \\
\text { fin }\end{array}$ & 7.9 & 3.2 & 6.4 & 0.7 & 3.3 & 2.8 & 3.3 \\
\hline Pectoral to pelvic & 1.1 & -1.7 & 6.3 & -2.4 & 2.9 & 0.7 & 3.4 \\
\hline Spine to first dorsal fin & 3.2 & 2.6 & 4.9 & -2.5 & 2.7 & -9.8 & 4.8 \\
\hline Clasper length total & 20.4 & 8.6 & 24.9 & NA & NA & 7.4 & 10.5 \\
\hline $\begin{array}{l}\text { Clasper length medial } \\
\text { branch }\end{array}$ & 5.8 & 1.3 & 6.6 & NA & NA & 0.8 & 2.0 \\
\hline $\begin{array}{l}\text { Clasper length lateral } \\
\text { branch }\end{array}$ & 7.4 & 1.5 & 8.3 & NA & NA & 0.9 & 3.6 \\
\hline Clasper length outer & 16.3 & 1.8 & 18.2 & NA & NA & 2.9 & 3.0 \\
\hline Clasper length inner & 19.1 & 6.1 & 21.3 & NA & NA & 5.3 & 5.8 \\
\hline Clasper width at base & 4.0 & 1.8 & 4.8 & NA & NA & 0.9 & 1.1 \\
\hline $\begin{array}{l}\text { Frontal tenaculum } \\
\text { length }\end{array}$ & 5.7 & 4.2 & 5.6 & NA & NA & 4.2 & 6.6 \\
\hline $\begin{array}{l}\text { Frontal tenaculum bulb } \\
\text { height }\end{array}$ & 1.7 & 0.0 & 1.9 & NA & NA & NA & NA \\
\hline $\begin{array}{l}\text { Frontal tenaculum bulb } \\
\text { length }\end{array}$ & 2.3 & 0.0 & 2.1 & NA & NA & NA & NA \\
\hline $\begin{array}{l}\text { Frontal tenaculum bulb } \\
\text { width }\end{array}$ & 2.2 & 1.6 & 1.9 & NA & NA & 1.2 & 1.6 \\
\hline $\begin{array}{l}\text { Frontal tenaculum stalk } \\
\text { width }\end{array}$ & N/A & 1.2 & 1.2 & NA & NA & 1.6 & 1.6 \\
\hline
\end{tabular}

First dorsal fin of moderate height, 20.3\% (16.2-19.1\%) BDL, triangular in shape, posterior margin slightly falcate, becomes strongly concave towards insertion into weblike interdorsal ridge and confluent to second dorsal fin. First dorsal fin proceeded by thick, fairly straight spine with slight posterior curve distally, extending past the apex of first dorsal fin, and extending to or slightly overlapping second dorsal fin origin when depressed against the body, overlap 7.9\%(0.7-6.4\%) BDL; spine anterior edge keeled, strongly trenchant, and marked with a dark brown line; two columns of serrations present 
on the distal one-third to one-half of the posterolateral edges of spine in mature individuals and entire length in smaller, immature specimens. Second dorsal fin about one-third height of first dorsal, elongated, base 74.8\% (70.1-77.5\%) BDL, without any distinct undulation mid-fin, anterior height $6.6 \%(3.8-7.2 \%)$ BDL slightly greater than middle height $5.9 \%(3.7-6.6 \%)$ BDL, and posterior height 5.5\% (4.1-6.6\%) BDL; fin inserts abruptly, rounding into a small lobe attached to caudal fin by a fleshy web; second dorsal fin somewhat feathery in appearance, easily splitting along radials.

Caudal fin very large, paddle-shaped, height approximately equal in upper and lower margins, dorsal caudal height 4.1\% (2.6-3.7\%) BDL, and ventral caudal height $4.1 \%$ (3.2-4.4\%) BDL, weakly raked from dorsal origin, tapers into a filament, often damaged in larger specimens; origin of caudal ventral margin is slightly anterior to origin of dorsal margin, connected to a small tab-like anal fin, which is proceeded by a fleshy ridge, dorsal caudal margin $26.6 \%(25.1-33.2 \%)$ BDL, and ventral caudal margin $36.0 \%$ $(39.2-57.3 \%)$ BDL.

Frontal tenaculum well developed on mature males, located medially on head, anterior and slightly dorsal to eyes; club-like, thick stalk length greater than one-half eye length, slightly curved, increasing in width distally, terminating in a bulbous tip. Bulb bearing spine-like denticles located on ventral distal surface of tip, varying in size, not in distinct rows, angled posteriorly (Figure 7a). Mature male with externally trifurcate, paired pelvic claspers, originating from muscular fin-base, transitioning distally into cartilaginous rod, total length $24.9 \%(2.7-24.9 \%)$ BDL, forked for at least distal onethird of length; pelvic claspers nearly reaching posterior margin of pelvic fins but not 
exceeding distal tip; intermediate branch thin, rod-like, surrounded by broad, soft, fleshy, dilated tip; two lateral branching arms broader, more robust, not widely separated, each with distal fleshy bulbous tip, soft with small denticles giving it a shagreen appearance (Figure 7b). Prepelvic tenacula paired, spatulate, with distal margin of hard structure deeply indented, and concealed within a slit-like pocket on ventral body surface just anterior to pelvic fins; each prepelvic tenacula with 4 large, tooth-like denticles tightly spaced along medial edge; numbered 1 through 4 distally, the second denticle is the largest, 1 and 3 of equal medium size, and 4 being the smallest (Figure 7c). Mature females with fleshy postanal pad, absent in males.
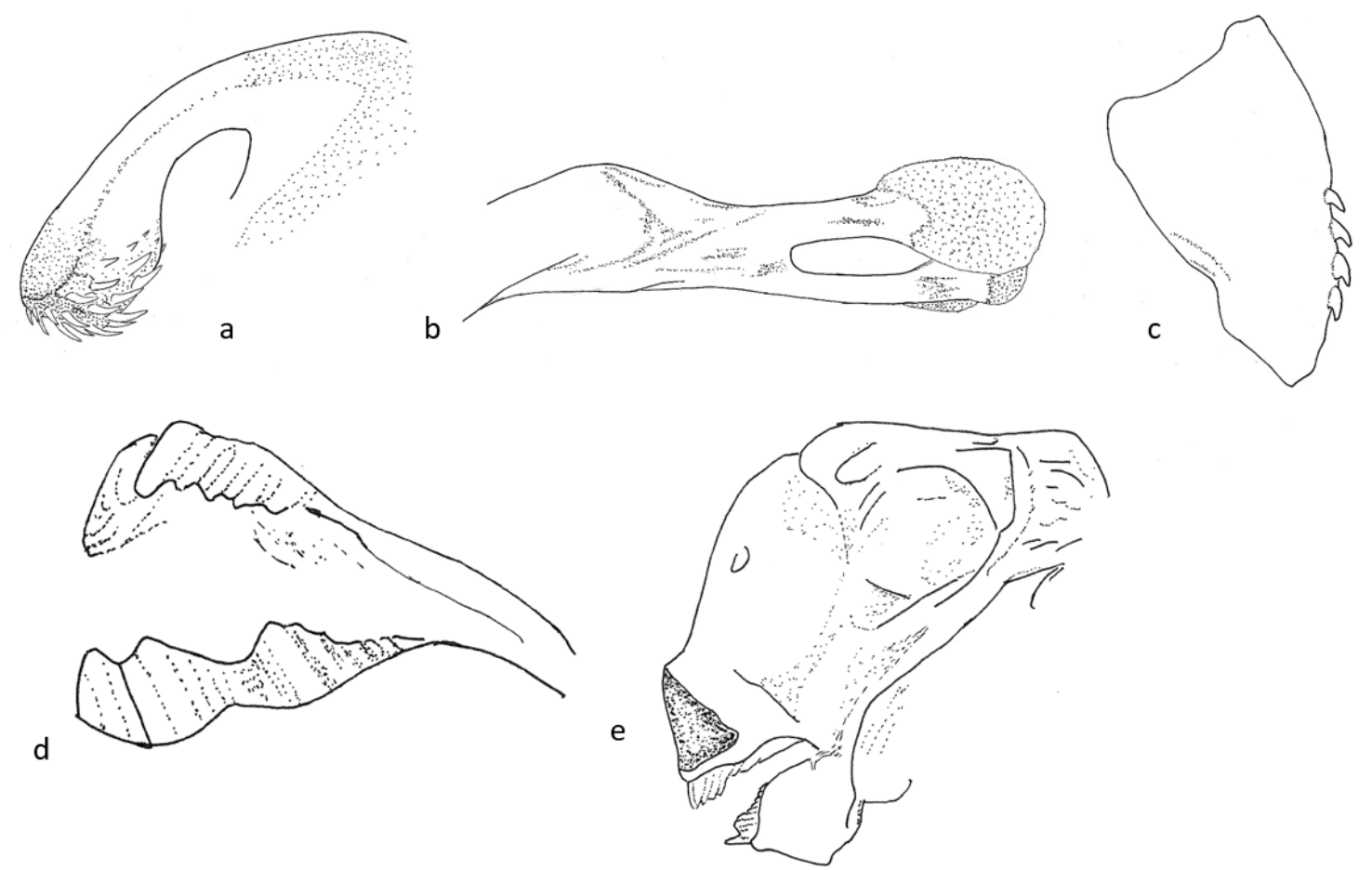

Figure 7. Chimaera willwatchi, sp. nov., holotype CAS 242336, mature male $834 \mathrm{~mm}$ TL, $492 \mathrm{~mm}$ BDL illustrations of a) Frontal tenaculum, b) Lateral view of pelvic claspers, c) Pre-pelvic tenacula, d) Tooth plates, and e) Skeletonized skull. Illustrations by P.J. Clerkin. 
Lateral lines of head open, narrow grooves, those on snout with wide regularly spaced dilations (Table 3). Preopercular and oral lateral line canals branching varies and is nondescriptive. Lateral line dips strongly just anterior of the spine origin, runs fairly straight along the length of the body and head (Figure 8).

Table 3. Lateral line canals of the head expressed as proportion of body length (\%BDL) of Chimaera willwatchi sp. nov.

\begin{tabular}{|c|c|c|c|c|c|c|c|}
\hline \multicolumn{8}{|c|}{ Chimaera willwatchi sp. nov. } \\
\hline & \multirow{4}{*}{$\begin{array}{c}\text { Holotype } \\
\mathrm{n}=1 \\
\text { Adult } \\
\text { Male }\end{array}$} & \multicolumn{2}{|c|}{ Paratype } & \multicolumn{2}{|c|}{ Paratype } & \multicolumn{2}{|c|}{ Nontype } \\
\hline & & \multicolumn{2}{|c|}{$n=5$} & \multicolumn{2}{|c|}{$\mathrm{n}=5$} & \multirow{3}{*}{\multicolumn{2}{|c|}{$\begin{array}{c}\mathrm{n}=45 \\
\text { (13 males, } 32 \\
\text { females) }\end{array}$}} \\
\hline & & \multirow{2}{*}{\multicolumn{2}{|c|}{ Male }} & \multirow{2}{*}{\multicolumn{2}{|c|}{ Female }} & & \\
\hline & & & & & & & \\
\hline & & Min & Max & Min & Max & Min & Max \\
\hline Body length (mm) & 492 & 403 & 490 & 519 & 604 & 118 & 285 \\
\hline $\begin{array}{l}\text { Measurement } \\
(\% \mathrm{BDL})\end{array}$ & & & & & & & \\
\hline $\begin{array}{l}\text { Oronasal to nasal } \\
\text { canal }\end{array}$ & 2.6 & 2.3 & 2.8 & 2.3 & 3.0 & 2.2 & 3.1 \\
\hline $\begin{array}{l}\text { Length of the rostral } \\
\text { canal }\end{array}$ & 1.5 & 1.1 & 1.5 & 0.9 & 1.0 & 1.3 & 2.0 \\
\hline $\begin{array}{l}\text { Length across nasal } \\
\text { canal }\end{array}$ & 6.6 & 6.4 & 8.1 & 4.5 & 8.2 & 6.2 & 7.6 \\
\hline $\begin{array}{l}\text { Infraorbital to angular } \\
\text { canal }\end{array}$ & 4.6 & 4.1 & 5.3 & 4.5 & 5.3 & 4.4 & 6.8 \\
\hline $\begin{array}{l}\text { Preopercular to main } \\
\text { trunk }\end{array}$ & 10.8 & 8.6 & 11.6 & 9.9 & 11.3 & 9.4 & 13.1 \\
\hline Orbital canal length & 5.0 & 1.4 & 5.9 & 4.1 & 5.2 & 4.1 & 6.3 \\
\hline $\begin{array}{l}\text { Supratemporal canal } \\
\text { length }\end{array}$ & 6.7 & 4.9 & 6.9 & 5.2 & 6.4 & 4.5 & 6.2 \\
\hline $\begin{array}{l}\text { Spine to } \\
\text { supratemporal canal }\end{array}$ & 4.3 & 3.7 & 4.8 & 4.0 & 5.2 & 4.2 & 7.0 \\
\hline
\end{tabular}




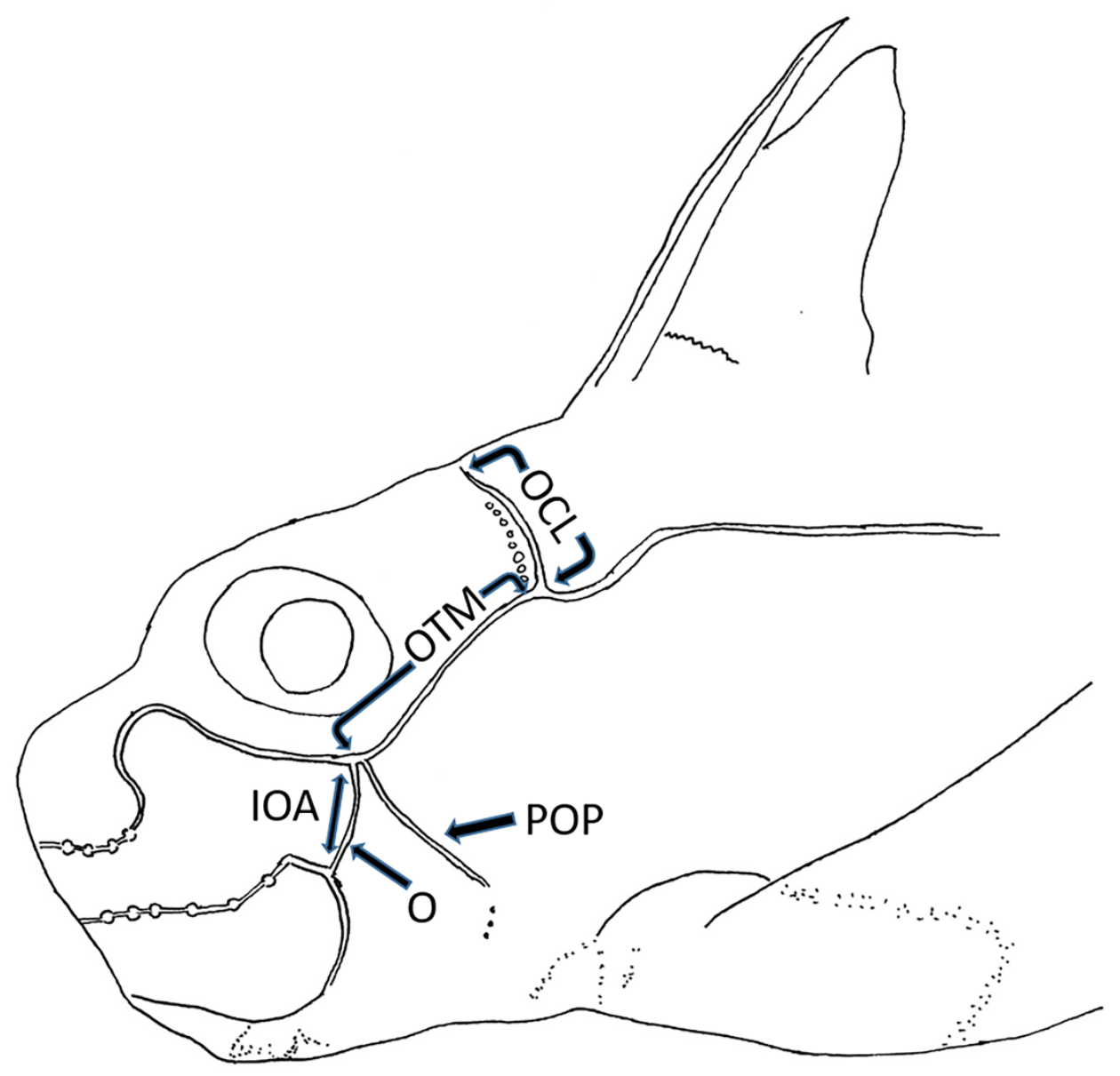

Figure 8. Illustration of Chimaera willwatchi, sp. nov., lateral lines of head showing: infraorbital to angular canal (IOA), junction of the oral-infraorbital canal to junction of the oral and angular canal, preopercular to main trunk (OTM), preopercular canal (POP), and oral canal (O). Illustration by P.J. Clerkin.

Ontogenetic Differences Between Large and Small Specimens. Chimaera willwatchi, sp. nov. appears to exhibit morphogenesis with immature differing from mature specimens in the following characteristics: greater total length (131.3\% vs $216.4 \%$ BDL), larger eye length (7.0\% vs $11.7 \%$ BDL) and eye height (5.1\% vs 9.2\% BDL), thinner snout base width (1.8\% vs $7.4 \%$ BDL), thinner snout anterior width (5.8\% vs $9.6 \%$ BDL), thinner head width at suborbital ridges (5.8\% vs 9.6\% BDL), thinner trunk width 
( $9.0 \%$ vs $16.2 \%$ BDL), thinner tail width ( $4.3 \%$ vs $8.7 \%$ BDL), longer abdomen $(28.8 \%$ vs $39.3 \% \mathrm{BDL}$ ) and tail lengths (46.8\% vs $56.3 \% \mathrm{BDL}$ ), smaller abdomen height (18.4 vs $26.8 \% \mathrm{BDL}$ ) and tail heights ( $12.0 \%$ vs $24.9 \% \mathrm{BDL}$ ), relatively larger pectoral fins (32.2\% vs $45.7 \% \mathrm{BDL})$, thicker spines, and longer first dorsal fin base (12.9\% vs $22.1 \%$ BDL). The smallest free-swimming individuals were white with translucent abdomens, but appear to gain pigmentation with growth (Figure 10c).

Dentition. Upper anterior tooth plates (vomerine) moderate, incisor-shaped, with 5 tridors per side, slightly overlapping mandibular tooth plates; posterior upper tooth plates (palatine) moderately large, flat, and ovular in shape; lower tooth plate (mandibular), moderately large, incisor-like, double cusps, transitions posteriorly into concave ridge resulting in 4 and 5 grinding surfaces and 11 tridors per side (Figure 8d).

Coloration. Three distinct color morphotypes were observed and each appears to separate spatially within the SWIO by major submarine geographical features (Figure 9); each color morph, here designated morphs A, B, and C, and its associated location are provided in the distribution section below (Tables 4 and 5). 


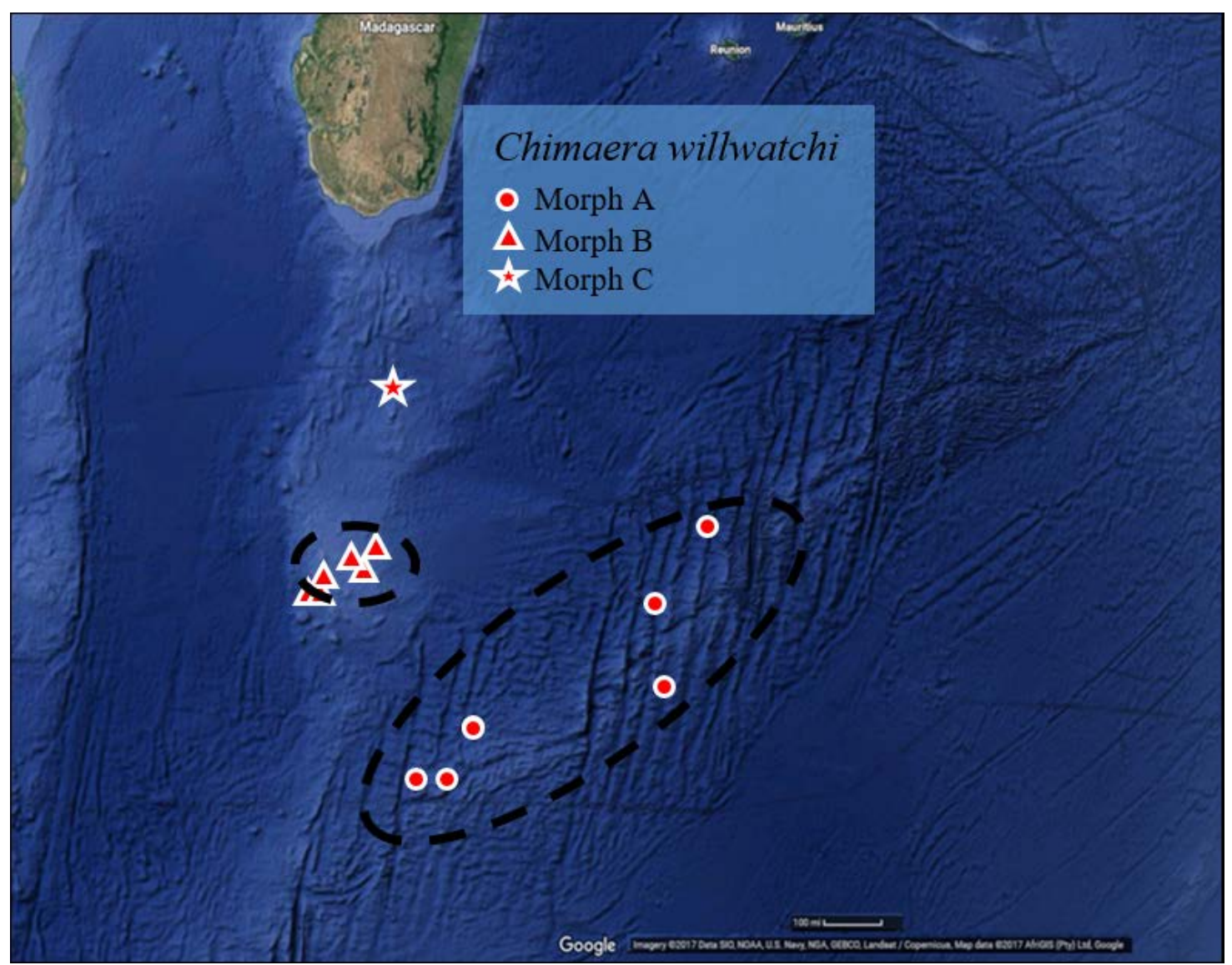

Figure 9. Map of study area denoting where Chimaera willwatchi, sp. nov., specimens were collected, with subpopulations represented by circles (morph A), star (morph B), and triangles (morph C). Map data: Google, Image (C) 2017 DigitalGlobe.

Morph A specimens prior to preservation are brownish-purple with iridescent-opal, oil-like sheen, brown and white mottling noticeably on snout, around mouth, white labials, and ventral half of trunk; tail brownish-purple, more uniform in color, with light and dark longitudinal striations (Figure 10). Fins purplish with a slightly grayish-brown and black speckling, dark radials, light basal border; thin dark shading where fin attaches to body; thick white margins very distinct on posterior margin of first dorsal fin and posterior margin of pelvic fins; a thin distinct white marking along anterior edge of second dorsal fin, extending up to one-half of fin margin length; white marking present, 
less distinct along posterior tips of dorsal caudal and ventral caudal fin margins. Lateral lines light in color and bordered by dark shading, running length of head and trunk. After preservation, colors fade, with purple luster and iridescence becoming diminished or lost.
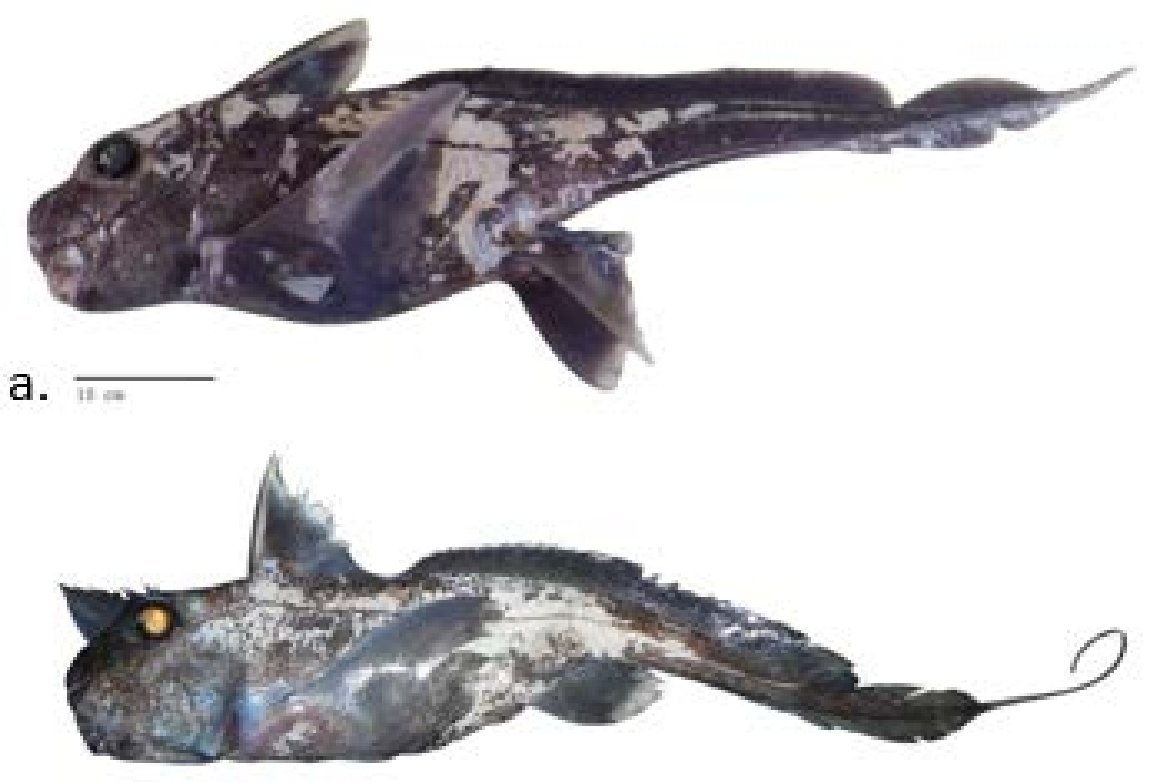

b. $10 \mathrm{~cm}$

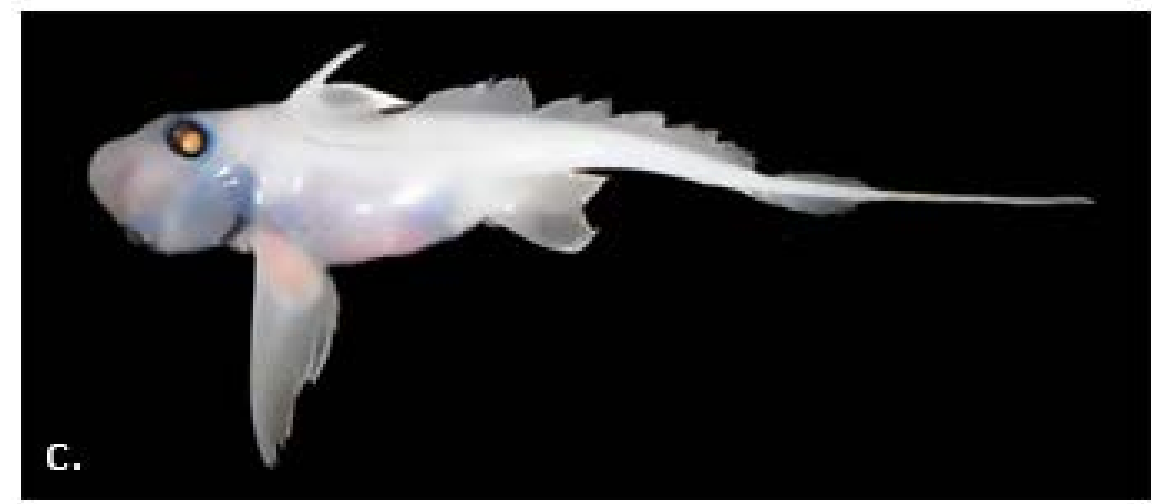

Figure 10. Chimaera willwatchi, sp. nov., morph A: a) holotype CAS 242336, mature male $834 \mathrm{~mm}$ TL, $492 \mathrm{~mm}$ BDL, b) paratype, immature male, $620 \mathrm{~mm}$ TL, $323 \mathrm{~mm}$ TL, c) paratype, newly hatched female, CAS 242351, $290 \mathrm{~mm}$ TL, $117.58 \mathrm{~mm}$ BDL. Photos by P.J. Clerkin. 
Morph B specimens are a uniform dark brown, with a slightly darker snout and dark lateral striations along tail, but lacking any distinctive markings, mottling, or speckles; fins dark brown-black, lacking white margins (Figure 11).

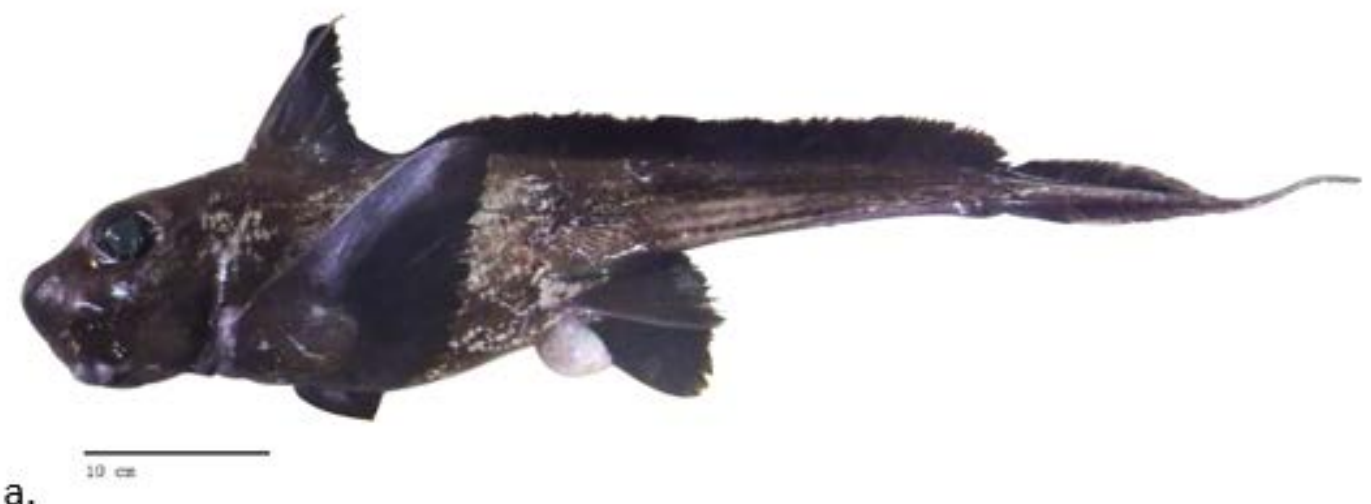

a.

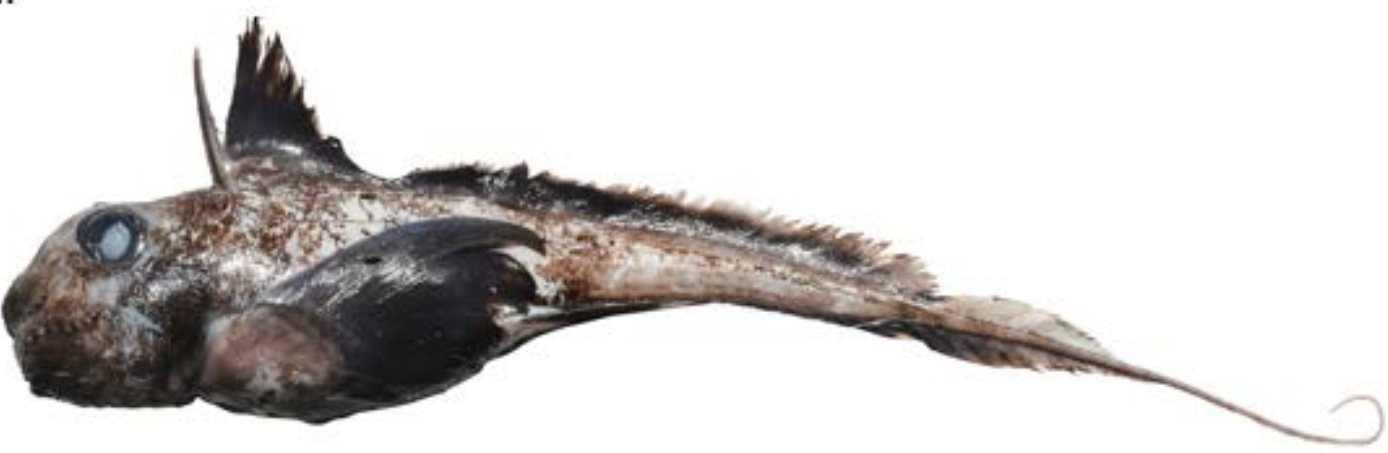

b. $10 \mathrm{~cm}$

Figure 11. Chimaera willwatchi, sp. nov., morph B: a) non-type CAS, immature male, $694 \mathrm{~mm}$ TL, 369 mm BDL, b) non-type CAS 242354, immature female, $477 \mathrm{~mm}$ TL, 252mm BDL. Photos by P.J. Clerkin.

Table 4. Raw measurements (in $\mathrm{mm}$ ) and body length proportions (\%BDL) of Chimaera willwatchi sp. nov. morphotypes B and C.

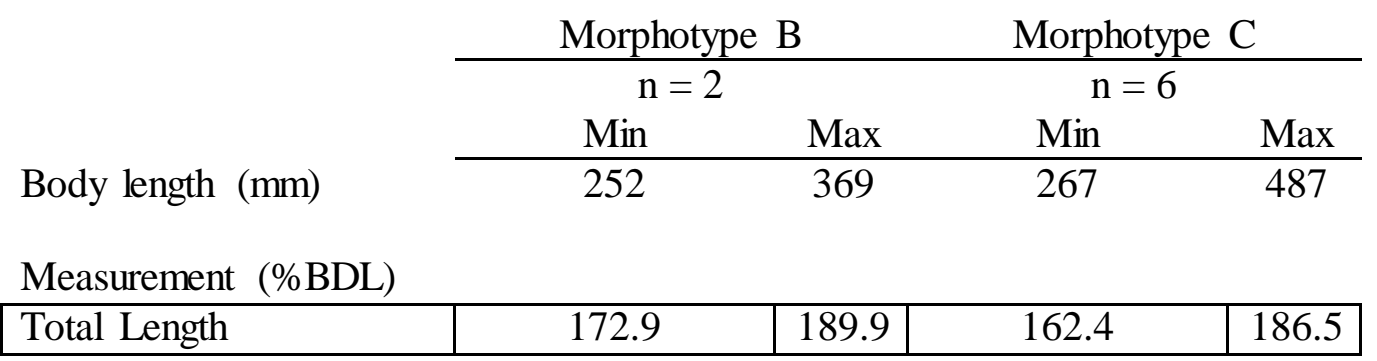




\begin{tabular}{|l|c|c|c|c|}
\hline Pre-caudal length & 53.5 & 123.8 & 119.3 & 126.1 \\
\hline Snout to vent length & 61.9 & 63.7 & 60.8 & 66.7 \\
\hline Tail length & 61.1 & 61.8 & 57.9 & 63.4 \\
\hline Trunk length & 40.0 & 43.8 & 41.1 & 44.7 \\
\hline Head length & 25.5 & 27.6 & 17.8 & 28.8 \\
\hline $\begin{array}{l}\text { Pre-first dorsal fin } \\
\text { length }\end{array}$ & 28.8 & 31.3 & 23.8 & 32.6 \\
\hline $\begin{array}{l}\text { Pre-second dorsal fin } \\
\text { length }\end{array}$ & 51.0 & 54.5 & 49.7 & 53.5 \\
\hline Pre-pectoral fin length & 29.8 & 29.8 & 6.1 & 34.6 \\
\hline Pre-pelvic fin length & 64.3 & 67.5 & 61.4 & 70.7 \\
\hline Pre-orbital length & 13.2 & 14.0 & 11.2 & 15.5 \\
\hline Pre-orbital distance & 13.6 & 15.2 & 12.3 & 16.1 \\
\hline Pre-narial length & 5.5 & 7.3 & 4.6 & 6.7 \\
\hline Pre-narial distance & 9.3 & 10.5 & 6.2 & 12.9 \\
\hline Pre-oral length & 5.1 & 7.2 & 5.4 & 9.0 \\
\hline Pre-oral distance & 12.8 & 13.7 & 9.2 & 14.5 \\
\hline Snout length & 10.8 & 11.2 & 5.2 & 11.9 \\
\hline Eye Length & 8.2 & 11.2 & 7.6 & 11.7 \\
\hline Eye Height & 6.3 & 8.1 & 5.5 & 9.2 \\
\hline First dorsal to pectoral & 18.7 & 25.5 & 15.3 & 25.5 \\
\hline First dorsal to pelvic & 38.9 & 42.9 & 39.5 & 43.4 \\
\hline $\begin{array}{l}\text { Second dorsal to } \\
\text { pectoral }\end{array}$ & 28.3 & 33.9 & 29.9 & \\
\hline Second dorsal to pelvic & 27.1 & 28.5 & 20.4 & 41.9 \\
\hline Snout width at base & 2.4 & 3.1 & 1.9 & 22.9 \\
\hline Snout anterior width & 9.5 & 9.6 & 5.8 & 8.2 \\
\hline $\begin{array}{l}\text { Head width at suborbital } \\
\text { ridge }\end{array}$ & 14.4 & 16.0 & 10.2 & 15.8 \\
\hline Trunk width & 15.3 & 16.2 & 10.9 & 16.0 \\
\hline Abdominal Width & 9.9 & 10.4 & 7.5 & 10.3 \\
\hline Tail width & 6.1 & 7.1 & 5.6 & 8.7 \\
\hline Cauldal peduncle width & 1.9 & 2.0 & 1.4 & 2.2 \\
\hline Snout height at base & 8.5 & 10.5 & 5.0 & 9.4 \\
\hline Head height & 24.7 & 27.3 & 22.5 & 26.7 \\
\hline Trunk height & 27.0 & 31.6 & 24.1 & 29.9 \\
\hline Max Trunk height & 21.1 & 28.4 & 22.9 & 29.1 \\
\hline Abdomen height & 14.4 & 21.8 & 18.4 & 23.0 \\
\hline Tail height & 15.9 & 15.9 & 12.0 & 16.3 \\
\hline Caudal peduncle height & 3.1 & 3.7 & 2.2 & 3.3 \\
\hline Interdorsal space & 6.2 & 7.6 & 4.7 & 10.0 \\
\hline Dorsal-caudal space & 0.8 & 1.3 & 0.6 & 2.0 \\
\hline
\end{tabular}




\begin{tabular}{|c|c|c|c|c|}
\hline Pectoral-pelvic space & 33.5 & 34.6 & 30.0 & 38.3 \\
\hline Pelvic-anal space & 47.5 & 48.3 & 38.9 & 49.8 \\
\hline Pelvic-caudal space & 52.9 & 54.7 & 46.8 & 54.5 \\
\hline Pelvic-ridge space & 20.9 & 22.3 & 16.2 & 23.6 \\
\hline $\begin{array}{l}\text { Pectoral fin anterior } \\
\text { margin }\end{array}$ & 36.2 & 40.1 & 32.2 & 39.4 \\
\hline Pectoral fin width & 23.6 & 23.6 & 19.1 & 24.9 \\
\hline Pectoral fin base width & 11.7 & 12.9 & 9.1 & 11.0 \\
\hline Pectoral fin base height & 12.3 & 12.5 & 10.1 & 13.2 \\
\hline Pelvic anterior margin & 23.2 & 24.8 & 20.2 & 24.3 \\
\hline Pelvic fin width & 14.3 & 14.5 & 11.8 & 15.1 \\
\hline Pelvic fin base width & 5.5 & 6.7 & 5.2 & 6.7 \\
\hline pelvic fin base height & 7.8 & 9.3 & 7.2 & 9.6 \\
\hline Dorsal spine length & 25.3 & 27.6 & 13.5 & 22.6 \\
\hline $\begin{array}{l}\text { Dorsal spine ridge to } \\
\text { origin }\end{array}$ & 3.9 & 4.0 & 2.7 & 3.9 \\
\hline First dorsal fin base & 16.7 & 19.2 & 14.5 & 17.5 \\
\hline First dorsal fin height & 18.3 & 18.4 & 15.6 & 20.4 \\
\hline Second dorsal fin base & 71.7 & 74.7 & 67.2 & 76.4 \\
\hline $\begin{array}{l}\text { Second dorsal fin } \\
\text { anterior height }\end{array}$ & 5.3 & 7.2 & 3.1 & 6.2 \\
\hline $\begin{array}{l}\text { Second dorsal fin } \\
\text { posterior height }\end{array}$ & 5.5 & 5.9 & 3.7 & 6.4 \\
\hline $\begin{array}{l}\text { Second dorsal fin mid } \\
\text { height }\end{array}$ & 6.3 & 7.3 & 4.4 & 6.9 \\
\hline Dorsal caudal margin & 27.7 & 31.2 & 21.9 & 30.2 \\
\hline Dorsal caudal height & 3.2 & 3.9 & 2.7 & 4.6 \\
\hline Ventral caudal margin & 33.9 & 44.9 & 29.4 & 40.5 \\
\hline $\begin{array}{l}\text { Fleshy ridge to caudal } \\
\text { insertion }\end{array}$ & 65.7 & 78.3 & 58.8 & 73.0 \\
\hline Ventral caudal height & 3.5 & 4.9 & 2.9 & 5.1 \\
\hline total caudal length & 49.8 & 63.0 & 40.9 & 60.8 \\
\hline $\begin{array}{l}\text { Spine to second dorsal } \\
\text { fin }\end{array}$ & 2.9 & 6.8 & -2.0 & 0.4 \\
\hline Pectoral to pelvic & 1.1 & 1.6 & -6.6 & 4.0 \\
\hline Spine to first dorsal fin & 2.4 & 2.6 & $\begin{array}{l}-1.4 \\
\end{array}$ & 0.0 \\
\hline Clasper length total & 7.8 & 10.5 & 0.0 & 12.4 \\
\hline $\begin{array}{l}\text { Clasper length medial } \\
\text { branch }\end{array}$ & 1.4 & 1.8 & 0.0 & 2.2 \\
\hline $\begin{array}{l}\text { Clasper length lateral } \\
\text { branch }\end{array}$ & 1.6 & 1.8 & 0.0 & 3.9 \\
\hline Clasper length outer & 2.5 & 3.0 & 0.0 & 6.1 \\
\hline Clasper length inner & 5.0 & 5.8 & 0 & 5.3 \\
\hline
\end{tabular}




\begin{tabular}{|l|c|c|c|c|}
\hline Clasper width at base & 1.1 & 1.2 & 0.0 & 1.2 \\
\hline $\begin{array}{l}\text { Frontal tenaculum } \\
\text { length }\end{array}$ & 4.3 & 6.6 & 0.0 & 4.2 \\
\hline $\begin{array}{l}\text { Frontal tenaculum bulb } \\
\text { height }\end{array}$ & NA & NA & NA & NA \\
\hline $\begin{array}{l}\text { Frontal tenaculum bulb } \\
\text { length }\end{array}$ & NA & NA & NA & NA \\
\hline $\begin{array}{l}\text { Frontal tenaculum bulb } \\
\text { width }\end{array}$ & 1.5 & 1.6 & 0.0 & 1.6 \\
\hline $\begin{array}{l}\text { Frontal tenaculum stalk } \\
\text { width }\end{array}$ & 1.5 & 1.6 & 0.0 & 0.0 \\
\hline
\end{tabular}

Table 5. Lateral line canals of the head expressed as proportion of body length (\%BDL) of Chimaera willwatchi sp. nov. morphotype B and C.

\begin{tabular}{|c|c|c|c|}
\hline \multicolumn{2}{|c|}{ Morphotype B } & \multicolumn{2}{|c|}{ Morphotype C } \\
\hline \multicolumn{2}{|c|}{$\mathrm{n}=2$} & \multicolumn{2}{|c|}{$n=6$} \\
\hline Min & Max & Min & Max \\
\hline 252 & 369 & 267 & 487 \\
\hline
\end{tabular}

\begin{tabular}{|l|c|c|c|c|}
\hline Oronasal to nasal canal & 3.1 & 4.6 & 2.2 & 2.4 \\
\hline Length of the rostral canal & 1.4 & 10.6 & 1.2 & 2.0 \\
\hline Length across nasal canal & 5.2 & 7.6 & 5.7 & 8.0 \\
\hline Infraorbital to angular canal & 5.2 & 5.7 & 4.0 & 9.3 \\
\hline Preopercular to main trunk & 4.0 & 11.5 & 4.0 & 10.6 \\
\hline Orbital canal length & 6.3 & 6.3 & 3.7 & 5.6 \\
\hline Supratemporal canal length & 5.8 & 5.8 & 4.2 & 5.9 \\
\hline Spine to supratemporal canal & 5.6 & 5.6 & 4.2 & 5.1 \\
\hline
\end{tabular}


Morph $\mathrm{C}$ is light beige in body color, speckled, and lighter ventrally, with fins blackpurple or light purple in color with dark margins (Figure 12).

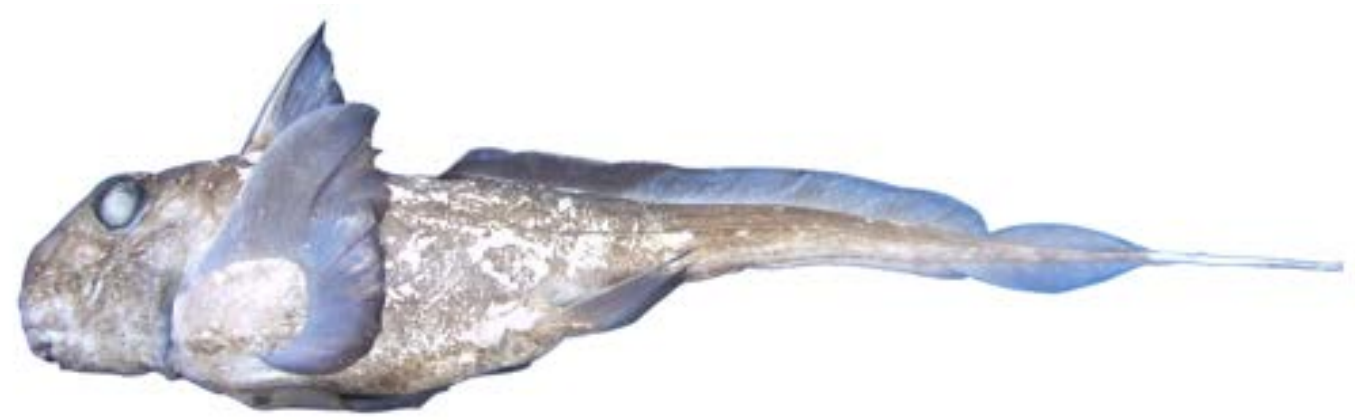

a. $10 \mathrm{em}$

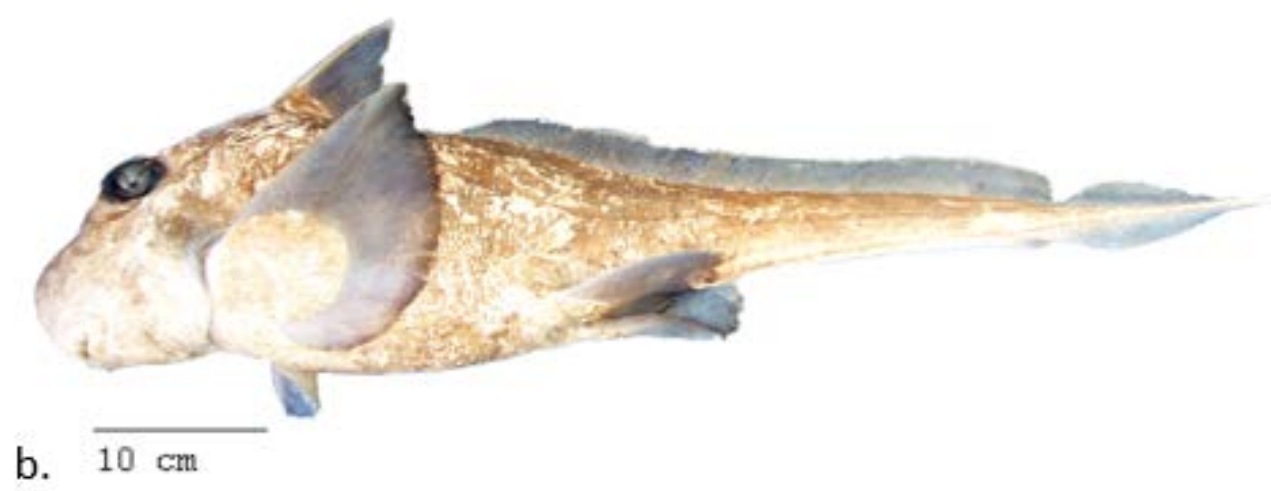

Figure 12. Chimaera willwatchi, sp. nov., morph C a) non-type CAS 242358, immature female, $975 \mathrm{~mm}$ TL, $542 \mathrm{~mm}$ BDL, b) Non-type CAS 242342, immature female, 620mm TL, 323 mm BDL. Photos by P.J. Clerkin.

Morph A was collected at all stages of development, whereas for morphs B and C only immature specimens were collected. However, similar-sized specimens from each morphotype were compared, and these coloration differences do not appear to be a function of ontogeny. Additionally, color pattern variation correlates strongly by region, and DNA sequence data group based on these locations (Figure 13). 


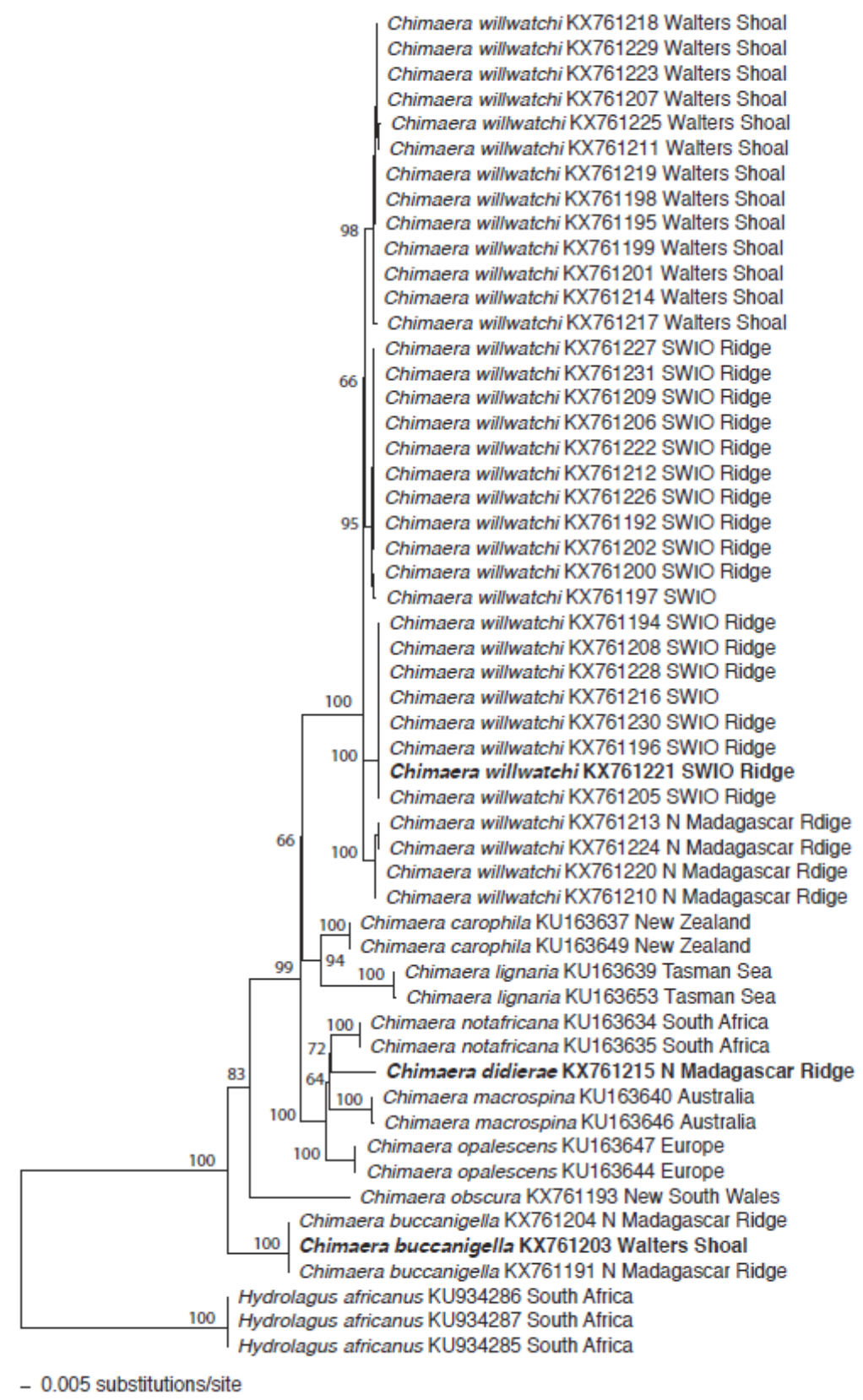

Figure 13. Maximum likelihood tree topology based on a general-time reversible (GTR) substitution model + gamma distribution for Chimaera willwatchi, sp. nov., Chimaera didierae, sp. nov., Chimaera buccanigella, sp. nov., and comparative species based on aligned NADH2 DNA sequences. GenBank accession numbers follow species (see Appendix B). Bootstrap support values of main clades shown on tree. Specimens indicated in bold are designated holotypes. 
Etymology. The new species is named in honor of the hard-working fishers onboard the Sealord fishing vessel Will Watch, on which the type specimens were collected. Vernacular: Seafarer's Ghost Shark.

Size. Maximum length for females is $645 \mathrm{~mm}$ BDL, 971+ mm TL, and for males 519 mm BDL, 834+ mm TL. Smallest free swimming individual 118 mm BDL, 258 mm TL. Females mature at $519 \mathrm{~mm}$ BDL, $767 \mathrm{~mm}$ TL, and males mature at $369 \mathrm{~mm}$ BDL, 638 mm TL.

Distribution. Chimaera willwatchi was encountered on all three main topographic features of the SWIO (Figure 9): Southwest Indian Ocean Ridge, northern portion of the

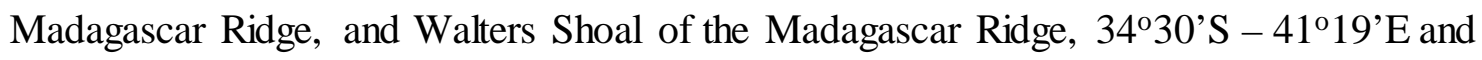
$39^{\circ} 50^{\prime} \mathrm{S}-5^{\circ} 15^{\prime} \mathrm{E}$; these areas are separated by roughly $600 \mathrm{~km}$ and $700 \mathrm{~km}$, respectively. However, each of these areas is represented by a different color morph of the species, which appears to be strongly spatially isolated by sub-region within the SWIO (Figure 9). Morph A was encountered most frequently, but only along the Southwest Indian Ocean Ridge, Morph B was taken from a single location in the northern part of the Madagascar Ridge, and Morph C was taken from seamounts around Walters Shoal on the southern part of the Madagascar Ridge. There was no overlap in the geographic ranges of each morph type.

Biological Notes. Specimens were collected from a wide depth range, $89 \mathrm{~m}-1365 \mathrm{~m}$, and encountered in both mid water and bottom trawls. However, there was no trend between depth range and morphotypes, perhaps due to confounding factors resulting from the large depth range within trawls. 
Comparisons. All comparisons of Chimaera willwatchi were made with specimens $\geq$ $400 \mathrm{~mm}$ BDL, representing mature and larger immature individuals. Chimaeroids exhibit considerable allometric changes with growth, with smaller immature specimens having proportionally different body dimensions than larger immature and mature specimens (Kemper et al., 2015). Therefore, removal of smaller immature individuals, those $<400$ mm BDL, allows for comparisons among similar sized individuals to better discern morphological differences across species; differentiation between species is indicated by either no overlap in a character range or a considerable difference in the minimum or maximum range of the character (Kemper et al., 2015). Morphometric data from 98 specimens, 10 species, two genera, and 29 parameters was analyzed in PRIMER to study morphometric features different between species (Table 6).

Chimaera willwatchi is mottled, but lacks strongly defined patterning of spots or reticulations, distinctly separating it from C. monstrosa, C. owstoni and C. panthera that, depending on the species, have distinct reticulations or spotting (Tanaka, 1905; Didier, 1998; Didier, et al., 2012; Ebert et al., 2013; Kemper et al., 2015). Chimaera willwatchi, although slightly iridescent, lacks any silvery body coloration, usually found in $C$.

argiloba, C. cubana, C. fulva, and C. phantasma (Jordan and Snyder, 1900; Didier et al., 2002, 2012).

Chimaera willwatchi is a large-bodied species (645 mm BDL), having a blocky head with well-defined suborbital ridges, blunt snout, and strong dorsal spine exceeding first dorsal apex. This combination of characters separates C. notafricana (its closest geographic congener) from $C$. willwatchi by its smaller head length, $21.5 \%(20.7-23.0 \%)$ 
BDL vs 27.0\% (23.0-28.9\%) BDL, shorter spine, 15.9-22.1\% BDL vs 22.9-27.3\% BDL, and shorter eye length, 6.3\%-6.5\% BDL vs. 8.5\% (7.6-9.2\%) BDL. Chimaera obscura is a smaller-bodied species (531 mm BDL), with a smaller head length, 24.5-25.0\% BDL vs 27.0\% (23.6-28.5\%) BDL, smaller eye length, 6.1-7.3\% BDL vs 8.5\% (7.7-9.2\%) BDL, less developed suborbital ridge, and a greater first dorsal fin height, $23.0-23.8 \%$ vs $20.3(18.0-19.1 \%)$ BDL that unlike $C$. willwatchi exceeds its dorsal spine height. Chimaera opalescens has a larger eye length to head ratio $(42.2 \%$ head length vs $31.6 \%$ head length), shorter dorsal spine, $12.4-20.2 \%$ vs $27.3 \%$ (22.9-24.9\%) BDL, spine height not exceeding first dorsal fin $(81.8-100 \%$ first dorsal fin height vs $133.9-173.1 \%$ first dorsal fin height). Chimaera bahamaensis is distinguished by its more pronounced snout, prenarial length $48.0 \%$ head length vs $14.8 \%$ (10.0-16.6\%) head length, preorbital length $62.0 \%$ head length vs $22.9 \%(16.1-31.8 \%)$ head length. Chimaera carophila is distinguishable by having a smaller head length, $22-24 \%$ BDL vs $27.0 \%(23.0-28.9 \%)$ BDL, greater eye length to head length ratio (32-39\% head length vs $26.2-31.6 \%$ head length), and shorter spine length, $18-20 \%$ BDL vs $27.3 \%$ (22.9-24.9\%) BDL. Chimaera jordani is similar to C. willwatchi, but has a smaller head length, $22.8 \%$ BDL vs 27.0\% (23.6-28.5\%) BDL, smaller head height 21.6\% BDL vs 26.6\% (23.0-26.7\%) BDL, and a longer trunk, 52.8\% BDL vs $43.1 \%$ (36.4-42.8\%) BDL, and smaller eye length, 6.6\% BDL vs 8.5\% (7.7-9.2\%) BDL.

The species most similar to $C$. willwatchi are $C$. lignaria, C. macrospina, and $C$. orientalis. Chimaera lignaria is most similar to $C$. willwatchi, having a huge blocky head, robust, stocky body quickly tapering to a long tail, and large fins. However, $C$. 
lignaria is known only from the Southwestern Pacific Ocean in the deep waters off New Zealand and Tasmania and is distinguishable from C. willwatchi in having a spine equal to or shorter than first dorsal fin, second dorsal fin rubbery in texture and not easily split, thin pectoral fins (broad in C. willwatchi), and rounded pelvic fins not pointed at the leading edge compared to the broad distally pointed pelvic fins of C. willwatchi. Chimaera lignaria is further separated from C. willwatchi by secondary sexual characters, having stout pelvic claspers reaching $17 \%$ BDL vs $24.9 \%$ BDL, bifurcate vs trifurcate, and prepelvic tenacula with 6 spines vs 4 in C. willwatchi. Chimaera marcospina has a less robust body, generally longer dorsal fin spine, and less broad; caudal fin shorter, ventral caudal margin $27.5-36 \%$ BDL vs $39.2-57.3 \%$ BDL, dorsal caudal fin height $1.7-3.2 \%$ BDL vs $2.6-4.1 \%$ BDL. Chimaera orientalis has a large head and body similar to C. willwatchi, but its fin proportions differ: pectoral fin anterior margin longer, $43.5 \%(40.0-41.0 \%)$ BDL vs $36.8 \%$ (33.7-40.2\%) BDL, pelvic fin anterior margin smaller, $22.2 \%(20.0-20.8 \%) \mathrm{BDL}$ vs $25.0 \%$ (22.9-26.8\%) BDL; first dorsal fin similar in height but with shorter base, $10.3-12.5 \%$ BDL vs $17.6 \%$ (14.2-17.9\%) BDL, a longer second dorsal fin base, $80.2 \%$ (79.2-81.3\%) BDL vs $74.8 \%$ (73.2-77.5\%) BDL; interdorsal fin space longer, $10.2 \%$ (8.1-9.7\%) BDL vs 3.3\% (4.6-7.2\%) BDL; claspers bifurcate, comparatively smaller, $17.5-17.7 \%$ BDL vs 20.4-24.9\% BDL, and with smaller frontal tenaculum, $4.7-4.6 \%$ BDL vs $5.6-5.7 \%$ BDL. 
Table 6. SIMPER pairwise comparison of morphometric measurements. Displayed are the top five ranked morphometric measurements for each species comparison; the contributing and cumulative percentages are expressed as \%.

\begin{tabular}{|c|c|c|c|}
\hline Species Comparisons & Measurement & $\begin{array}{l}\text { Contributing } \\
\text { Percent (\%) } \\
\end{array}$ & $\begin{array}{l}\text { Cumulative } \\
\text { Percent } \\
(\%)\end{array}$ \\
\hline \multirow[t]{5}{*}{$\begin{array}{l}\text { C. willwatchi and } C \text {. } \\
\text { notafricana }\end{array}$} & $\begin{array}{l}\text { Pelvic anterior } \\
\text { margin }\end{array}$ & 6.87 & \\
\hline & $\begin{array}{l}\text { Head width at } \\
\text { suborbital ridge }\end{array}$ & 6.44 & \\
\hline & Pectoral fin width & 6.08 & \\
\hline & Pelvic fin width & 5.43 & \\
\hline & $\begin{array}{l}\text { Preopercular to main } \\
\text { trunk }\end{array}$ & 5.22 & 30.76 \\
\hline \multirow[t]{5}{*}{$\begin{array}{l}\text { C. willwatchi and } C \text {. } \\
\text { opalescens }\end{array}$} & $\begin{array}{l}\text { Supratemporal canal } \\
\text { length }\end{array}$ & 8.53 & \\
\hline & Trunk width & 7.47 & \\
\hline & Snout length & 7.47 & \\
\hline & Dorsal caudal height & 6.51 & \\
\hline & $\begin{array}{l}\text { Length of the rostral } \\
\text { canal }\end{array}$ & 5.50 & 35.48 \\
\hline \multirow[t]{5}{*}{$\begin{array}{l}\text { C. willwatchi and } C \text {. } \\
\text { opalescens }\end{array}$} & $\begin{array}{l}\text { Supratemporal canal } \\
\text { length }\end{array}$ & 8.53 & \\
\hline & Trunk width & 7.47 & \\
\hline & Snout length & 7.47 & \\
\hline & Dorsal caudal height & 6.51 & \\
\hline & $\begin{array}{l}\text { Length of the rostral } \\
\text { canal }\end{array}$ & 5.50 & 35.48 \\
\hline \multirow{5}{*}{$\begin{array}{l}\text { C. willwatchi and } C \text {. } \\
\text { phantasma }\end{array}$} & Ventral caudal height & 12.84 & \\
\hline & Trunk length & 9.20 & \\
\hline & Pectoral fin width & 7.70 & \\
\hline & $\begin{array}{l}\text { Pectoral fin anterior } \\
\text { margin }\end{array}$ & 7.22 & \\
\hline & $\begin{array}{l}\text { Length of the rostral } \\
\text { canal }\end{array}$ & 5.84 & 42.80 \\
\hline \multirow[t]{4}{*}{ C. willwatchi and $H$. affinis } & Eye Length & 7.32 & \\
\hline & $\begin{array}{l}\text { Oronasal to nasal } \\
\text { canal }\end{array}$ & 5.68 & \\
\hline & $\begin{array}{l}\text { Second dorsal fin } \\
\text { anterior height }\end{array}$ & 4.99 & \\
\hline & Ventral caudal height & 4.82 & \\
\hline
\end{tabular}




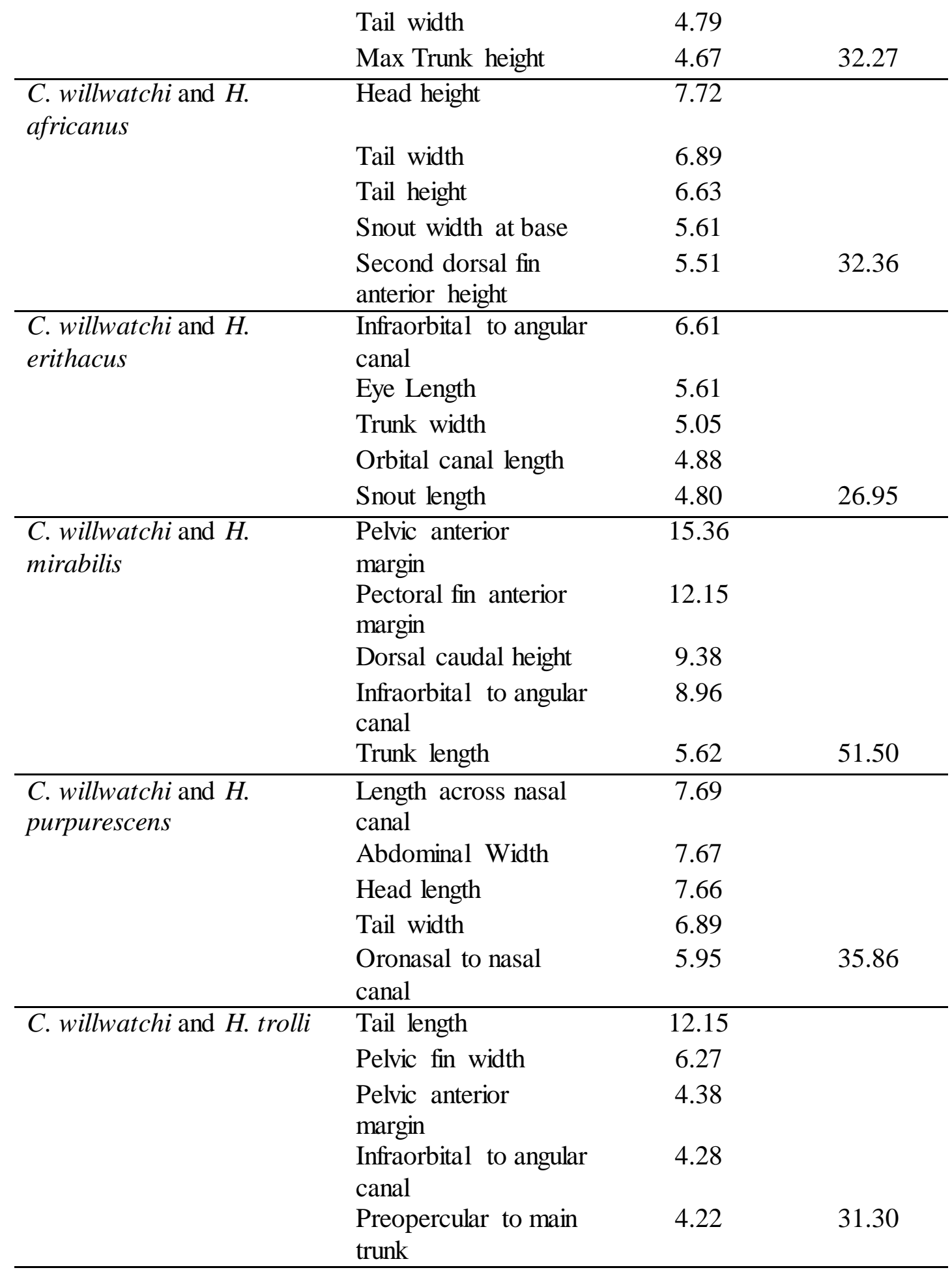


Chimaera willwatchi is the sixth species of chimaera known from the Indian Ocean, but only the second species known to occur in the Western Indian Ocean; this total excludes the two new species described below. The only other Chimaera species known to occur in the Western Indian Ocean is C. notafricana, which just barely ranges into the Southwestern Indian Ocean, to Algoa Bay, Eastern Cape Province, South Africa; this species is most common in the Southeastern Atlantic off the west coast of South Africa and Namibia (Kemper et al., 2010a; Ebert, 2014, 2015). All the other four species, $C$. argiloba, C. fulva, C. lignaria, and C. macrospina, are only known from the Eastern Indian Ocean and Southwestern Pacific Ocean (Ebert, 2014).

In addition to its morphometric and meristic distinction, C. willwatchi is the only Chimaera species with the unique combination of iridescent sheen when fresh, distinct mottling around mouth, and ventral trunk, very conspicuous white marking on posterior margin of first dorsal fin, anterior edge of second dorsal fin, and posterior margin of pelvic fins. Mottling and white margins are only found in certain geographic populations, and while they are useful to positively identify the species, their absence cannot be relied upon to discount a specimen as C. willwatchi, and should be used in combination with morphometric measurements (Figure 14). 
Euclidean Distance (Normalized) of Chimaeroid Morphometrics

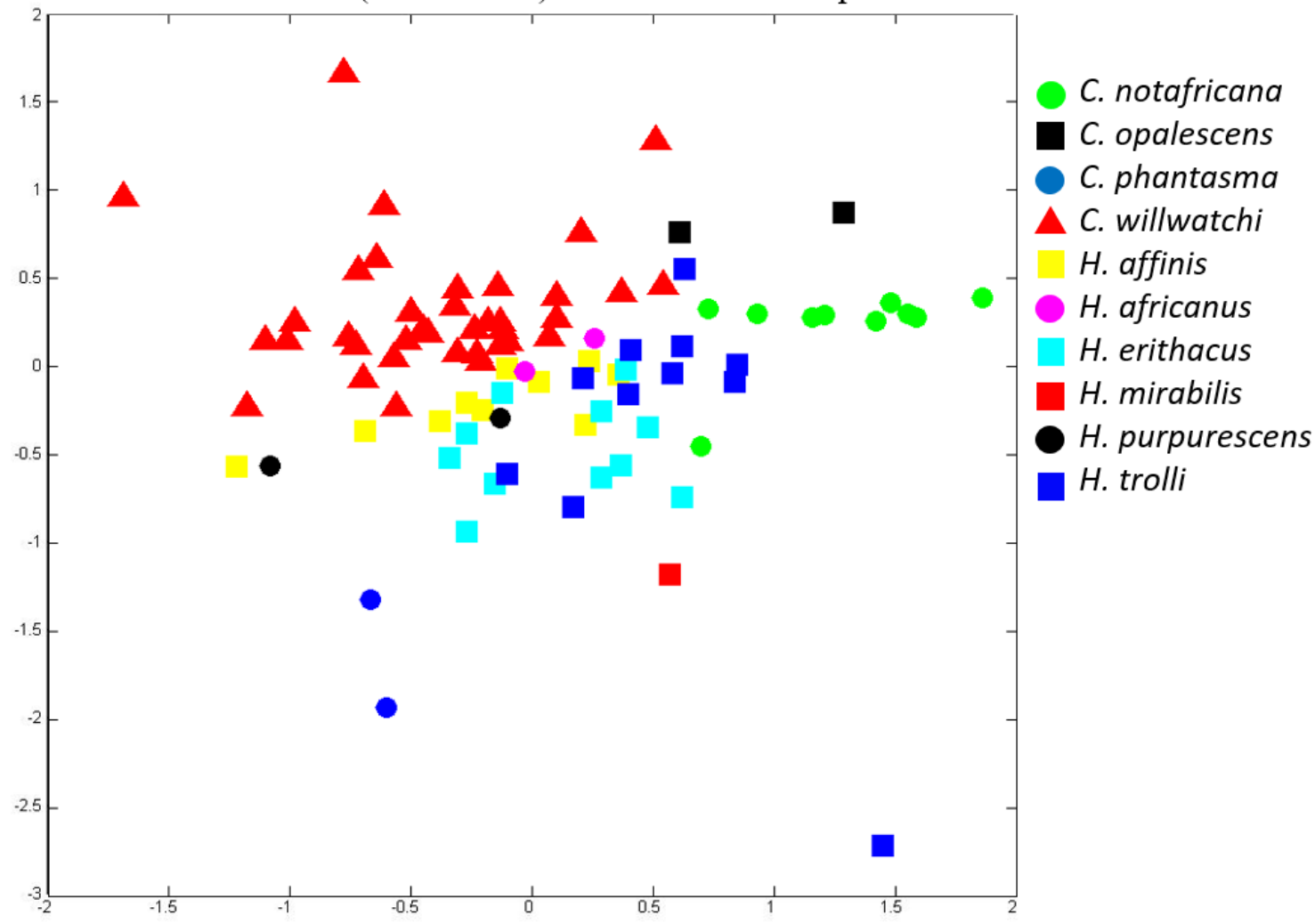

Figure 14. Normalized Euclidean distances illustrating morphometric dissimilarities between 10 chimaeroid species.

\section{Chimaera didierae, sp. nov., The Falkor Chimaera}

Holotype. CAS 242334, 825 mm TL, 533 mm BDL, female, Southwestern Indian Ocean, Madagascar Ridge, 34³0’S, 43¹0’E, bottom trawl between 1000 m-1100 m, collector P.J. Clerkin, 10 March 2012.

Diagnosis. Chimaera didierae, sp. nov., is the seventh species of chimaera known from the Indian Ocean, and is distinguishable from other members of the genus by the following combination of characters: medium-bodied slender species, short trunk, long tail, with moderately sized head, large eyes, without well-defined suborbital ridge; short 
thin snout; uniform light toffee-tan color, not iridescent, without defined patterning, spots, reticulations, or mottling, dark, sooty brown on snout, white blotching around mouth; pectoral fins long and thin, anterior margin 33.7\% BDL, pectoral fin width $16.7 \%$ BDL; dorsal spine height $>21.6 \%$ BDL, equal or subequal to height of first dorsal fin; second dorsal fin very long and low, anterior height 2.7\% BDL, not undulating; caudal fin small and slender, dorsal caudal height 1.8\% BDL, ventral caudal height 1.1\% BDL. Structure of the NADH2 gene. Chimaera didierae, sp. nov. can be distinguished from its morphologically similar congeners, Chimaera obscura Didier et al. 2008, and Chimaera opalescens Luchetti et al. 2001, by the combination of characters: relatively short dorsal spine, short first dorsal fin height, short second dorsal fin, small pectoral fin, moderately large eye length, abdomen tapering rapidly into tail, relatively long snout length, and coloration.

Description. Morphometric proportions of holotype are provided in Table 7. A medium-bodied species, moderate sized head length 24.2\% BDL, relatively tall head height, 20.5\% BDL, suborbital ridge not well-defined; snout moderately long relative to head, 40.3\% head length, and thin, width 2.0\% BDL and 8.2\% head length. Trunk slightly compressed, trunk height $25.7 \%$ BDL, tapering slightly to abdomen, height 19.9\% BDL, before tapering somewhat rapidly into tail, height $12.4 \% \mathrm{BDL}$, continuing into caudal filament. Tail long making up 52.6\% precaudal length, short trunk, 33.2\% precaudal length, relatively long head, 20.0\% precaudal length. Eyes large $8.3 \%$ BDL making up one-third (33.9\%) of head length, and ovoid, located in posterior half of head, preorbital length 52.4\% head length. Interdorsal space moderate, 8.3\% BDL, pectoral- 
anal space 32.1\% BDL, a little more than half (60\%) pelvic anal space, 53.8\% BDL. Pectoral-pelvic space 32.0\% BDL, 1.3 times head length, and is shorter than pelvic caudal space. Pelvic-caudal space 57.3\% BDL, about 2.3 times head length, and is approximately equal to snout-vent length 59.4\% BDL, 2.5 times head length. Skin smooth without denticles, strongly deciduous. 
Table 7. Body length proportions (\%BDL) of Chimaera didierae sp. Nov and Chimaera buccanigella sp. nov.

\begin{tabular}{|c|c|c|}
\hline & $\begin{array}{l}\text { Chimaera didierae } \\
\text { sp. nov }\end{array}$ & $\begin{array}{l}\text { Chimaera buccanigella } \\
\text { sp. Nov }\end{array}$ \\
\hline & Holotype & Holotype \\
\hline & $\mathrm{n}=1$ & $n=1$ \\
\hline & Female & Female \\
\hline Body length & $532 \mathrm{~mm}$ & $397 \mathrm{~mm}$ \\
\hline Measurement & \%BDL & \%BDL \\
\hline Total Length & 155.5 & 192.4 \\
\hline Pre-caudal length & 121.1 & 125.4 \\
\hline Snout to vent length & 59.4 & 67.3 \\
\hline Tail length & 63.7 & 60.5 \\
\hline Trunk length & 40.2 & 46.2 \\
\hline Head length & 24.2 & 28.3 \\
\hline Pre-first dorsal fin length & 25.0 & 29.5 \\
\hline $\begin{array}{l}\text { Pre-second dorsal fin } \\
\text { length }\end{array}$ & 46.2 & 45.0 \\
\hline Pre-pectoral fin length & 28.0 & 32.9 \\
\hline Pre-pelvic fin length & 61.1 & 70.1 \\
\hline Pre-orbital length & 12.5 & 14.8 \\
\hline Pre-orbital distance & 12.8 & 14.8 \\
\hline Pre-narial length & 13.6 & 9.1 \\
\hline Pre-narial distance & 9.7 & 11.8 \\
\hline Pre-oral length & 9.0 & 11.7 \\
\hline Pre-oral distance & 13.1 & 16.7 \\
\hline Snout length & 10.3 & 13.1 \\
\hline Eye Length & 8.3 & 9.6 \\
\hline Eye Height & 6.0 & 6.6 \\
\hline First dorsal to pectoral & 16.0 & 20.1 \\
\hline First dorsal to pelvic & 41.6 & 45.8 \\
\hline Second dorsal to pectoral & 24.4 & 23.9 \\
\hline Second dorsal to pelvic & 23.1 & 29.9 \\
\hline Snout width at base & 2.0 & 2.0 \\
\hline Snout anterior width & 6.5 & 7.1 \\
\hline $\begin{array}{l}\text { Head width at suborbital } \\
\text { ridge }\end{array}$ & 12.0 & 14.5 \\
\hline Trunk width & 11.1 & 12.6 \\
\hline Abdominal Width & 6.4 & 7.5 \\
\hline Tail width & 5.8 & 7.3 \\
\hline Caudal peduncle width & 1.5 & 1.7 \\
\hline Snout height at base & 7.5 & 9.5 \\
\hline
\end{tabular}




\begin{tabular}{|c|c|c|}
\hline Head height & 20.5 & 21.4 \\
\hline Trunk height & 25.7 & 24.9 \\
\hline Max Trunk height & 24.8 & 24.1 \\
\hline Abdomen height & 19.9 & 24.7 \\
\hline Tail height & 12.4 & 14.6 \\
\hline Caudal peduncle height & 2.3 & 3.3 \\
\hline Interdorsal space & 8.5 & 2.7 \\
\hline Dorsal-caudal space & 1.4 & 0.7 \\
\hline Pectoral-pelvic space & 32.0 & 34.8 \\
\hline Pelvic-anal space & 55.3 & 45.8 \\
\hline Pelvic-caudal space & 57.3 & 46.6 \\
\hline Pelvic-ridge space & 21.8 & 18.8 \\
\hline $\begin{array}{l}\text { Pectoral fin anterior } \\
\text { margin }\end{array}$ & 33.7 & 36.6 \\
\hline Pectoral fin width & 16.7 & 18.5 \\
\hline Pectoral fin base width & 10.2 & 10.1 \\
\hline Pectoral fin base height & 12.4 & 15.3 \\
\hline Pelvic anterior margin & 19.9 & 22.1 \\
\hline Pelvic fin width & 11.4 & 10.5 \\
\hline Pelvic fin base width & 4.8 & 6.2 \\
\hline pelvic fin base height & 7.0 & 7.2 \\
\hline Dorsal spine length & $21.6^{+}$ & 23.4 \\
\hline $\begin{array}{l}\text { Dorsal spine ridge to } \\
\text { origin }\end{array}$ & 2.8 & 3.1 \\
\hline First dorsal fin base & 13.2 & 16.1 \\
\hline First dorsal fin height & 12.1 & 15.5 \\
\hline Second dorsal fin base & 77.1 & 81.9 \\
\hline $\begin{array}{l}\text { Second dorsal fin anterior } \\
\text { height }\end{array}$ & 2.7 & 3.4 \\
\hline $\begin{array}{l}\text { Second dorsal fin } \\
\text { posterior height }\end{array}$ & 2.1 & 3.5 \\
\hline $\begin{array}{l}\text { Second dorsal fin mid } \\
\text { height }\end{array}$ & 2.1 & 3.5 \\
\hline Dorsal caudal margin & 21.4 & 20.1 \\
\hline Dorsal caudal height & 1.8 & 2.4 \\
\hline Ventral caudal margin & 22.7 & 28.2 \\
\hline $\begin{array}{l}\text { Fleshy ridge to caudal } \\
\text { insertion }\end{array}$ & 58.8 & 60.2 \\
\hline Ventral caudal height & 1.1 & 2.6 \\
\hline total caudal length & 29.9 & 66.8 \\
\hline Spine to second dorsal fin & 1.0 & 5.2 \\
\hline Pectoral to pelvic & 2.0 & -0.5 \\
\hline
\end{tabular}


Pectoral fins relatively narrow, pectoral fin width $16.7 \%$, long anterior margin, $33.7 \%$ BDL, about 1.4 times (139.3\%) head length; triangular in shape, rounded near base, anterior margin sigmoidal in shape, slightly concave near origin, increasing convexity about mid-length of anterior margin, increasing curvature distally to an apex, posterior margin slightly concave, rounded towards base. Pectoral fins, when depressed posteriorly against the body, barely reach the origin of pelvic fins, overlapping by $2.0 \%$ BDL. Pectoral fin base fleshy, rounded, asymmetrical in shape. Pelvic fins about half size (59\%) of pectoral fins, smaller than (82.3\%) head length, anterior margin very convex, posterior margin straight, rounding sharply into base, tear-drop in shape, tapering to a point distally; fleshy base thin and oval in shape. 


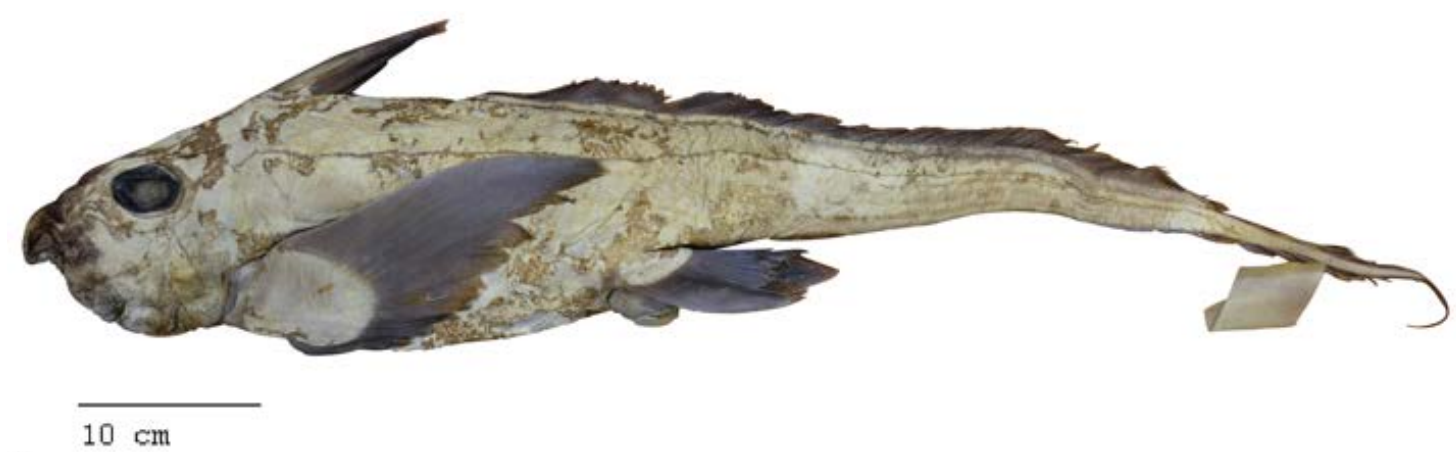

a.

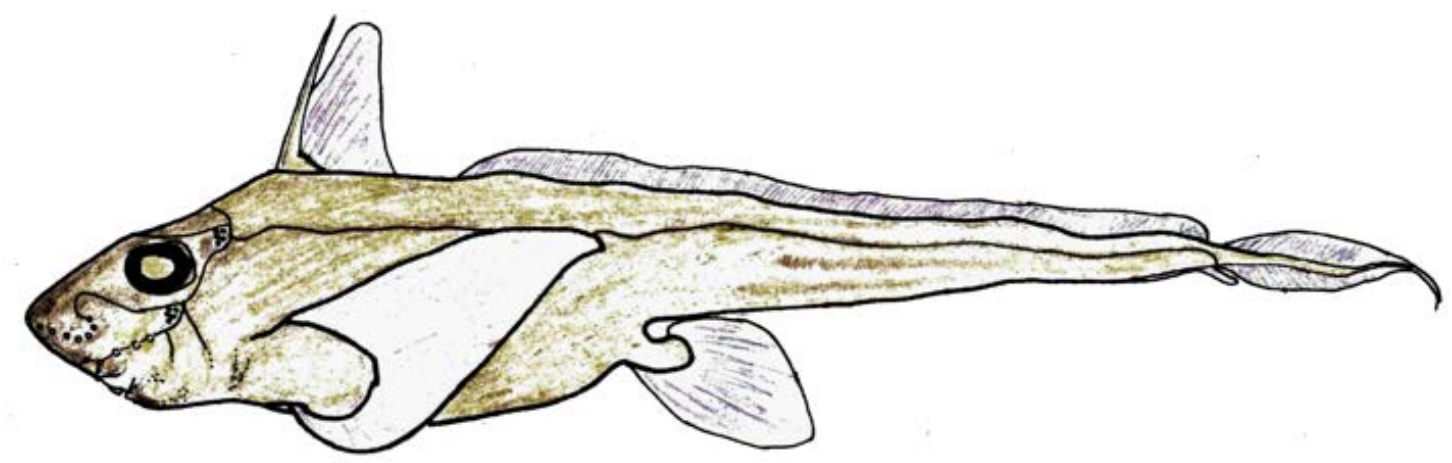

b.

Figure 15. Full body lateral Chimaera didierae, sp. nov., holotype CAS 242334, immature female, $890 \mathrm{~mm}$ TL, $704 \mathrm{~mm}$ BDL, $532 \mathrm{~mm}$ PCL a) photograph, b) illustration. Photo and illustration by P.J. Clerkin.

First dorsal fin very small, height 12.0\% BDL, base 14.0\% BDL, triangular in shape, and proceeded by relatively tall, $>21.4 \% \mathrm{BDL}$, robust spine. Spine ridge to origin $2.8 \%$ BDL, slightly posteriorly curved spine, curvature occurring evenly throughout spine, spine height equal or subequal to apex of first dorsal fin. Spine triangular in cross section, keel strongly trenchant along anterior edge, and two closely spaced columns of serrations along distal one-fourth of posterolateral edges. Posterior edge connects to first dorsal fin for approximately half of spine's length. Dorsal spine originates just posterior to head, pre-first dorsal length $25.0 \% \mathrm{BDL}$, just anterior to pectoral fin origin, and when 
depressed against the body, barely reaches second dorsal fin origin, overlaps by $2.0 \%$ BDL. Second dorsal fin less than one-fourth (23.8\%) height of first dorsal fin, elongate, base 77.0\% BDL, low, second dorsal fin anterior height 2.7\% BDL, and not noticeably undulating. Caudal fin small and slender, dorsal caudal margin 21.4\% BDL, nearly equal to ventral caudal margin, $22.7 \%$ BDL; with very low dorsal caudal height, $1.8 \% \mathrm{BDL}$, nearly equal to ventral caudal height, $1.1 \% \mathrm{BDL}$; dorsal and ventral margins of caudal fin originate at roughly the same position horizontally; caudal fin tapers off very gradually and ends in a thin, short filament. Anal fin very thin but long. Edges of fins feathery, jagged, and easily frayed.

Lateral line measurements of holotype are presented in Table 8. Lateral lines of head open, narrow grooves, those on snout with sparse dilations. Preopercular and oral lateral line canals share a common branch connecting to the infraorbital canal on both sides of head. Supratemporal canal not fully connected to dorsal spine.

Table 8. Lateral line canals of the head expressed as proportion of body length (\%BDL) of Chimaera didierae sp. nov and Chimaera buccanigella sp. nov.

\begin{tabular}{lcc}
\hline & \multicolumn{1}{c}{$\begin{array}{c}\text { Chimaera didierae } \\
\text { sp. nov }\end{array}$} & $\begin{array}{c}\text { Chimaera buccanigella } \\
\text { sp. nov }\end{array}$ \\
& \multicolumn{1}{c}{ Holotype } & Holotype \\
\cline { 2 - 3 } & $\mathrm{n}=1$ & $\mathrm{n}=1$ \\
& Female & Female \\
Body length & $532 \mathrm{~mm}$ & $397 \mathrm{~mm}$ \\
Measurement & $\% \mathrm{BDL}$ & $\% \mathrm{BDL}$ \\
\hline Oronasal to nasal canal & 2.3 & 2.0 \\
\hline Length of the rostral & 1.8 & 2.1 \\
canal & & \\
\hline
\end{tabular}




\begin{tabular}{|l|c|c|}
$\begin{array}{l}\text { Length across nasal } \\
\text { canal }\end{array}$ & 4.9 & 5.1 \\
\hline $\begin{array}{l}\text { Infraorbital to angular } \\
\text { canal }\end{array}$ & 3.1 & 8.9 \\
\hline $\begin{array}{l}\text { Preopercular to main } \\
\text { trunk }\end{array}$ & 7.9 & 9.4 \\
\hline Orbital canal length & 4.4 & 5.3 \\
\hline $\begin{array}{l}\text { Supratemporal canal } \\
\text { length }\end{array}$ & 4.8 & 5.1 \\
\hline $\begin{array}{l}\text { Spine to supratemporal } \\
\text { canal }\end{array}$ & 3.3 & 3.9 \\
\hline
\end{tabular}

Anterior origin of trunk lateral lines branches from junction of occipital and optic canals. Lateral line dips sharply ventrally then dorsally in a sigmoidal curve before returning to a relatively stable line at origin of dorsal spine and continuing posteriorly relatively non-undulating. Lateral line canal originating at fork between occipital and optic head canals at level of upper eye margin. Orbital canal length $4.4 \%$ BDL, and joins a short supratemporal canal, $4.8 \%$ BDL, which curves anteriorly to where it joins at dorsal midline $3.3 \%$ BDL anterior of dorsal spine origin. Infraorbital to angular canal short, 3.1\% BDL, and directed posteriordorsally to the junction of the oral and angular canal, where it continues to main trunk, preopercular to main truck, 7.9\% BDL. 


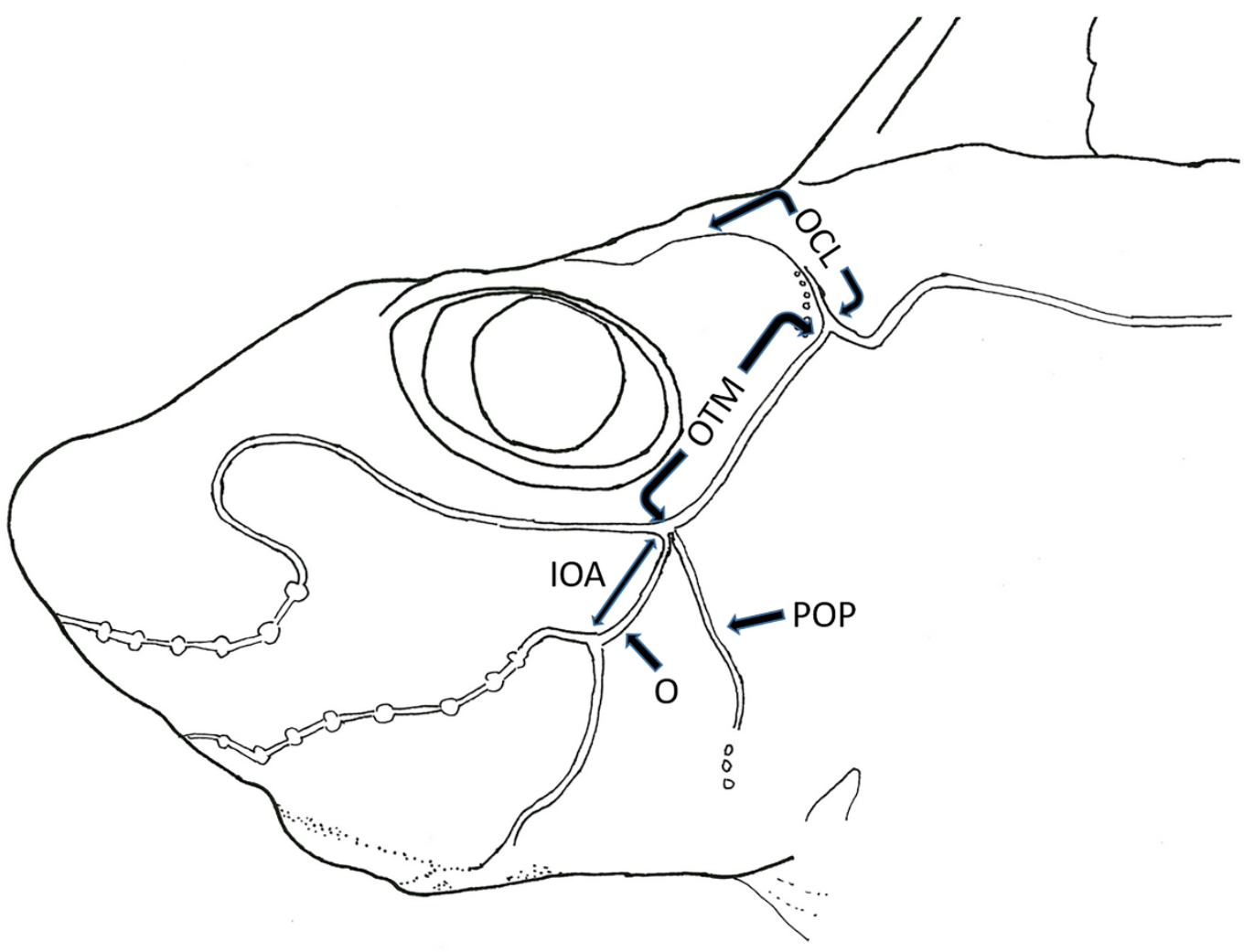

Figure 16. Illustration of Chimaera didierae, sp. nov., lateral lines of head showing: infraorbital to angular canal (IOA), junction of the oral-infraorbital canal to junction of the oral and angular canal, preopercular to main trunk (OTM), preopercular canal (POP), and oral canal (O). Illustration by P.J. Clerkin.

Dentition. In order to preserve the integrity of the unique specimen, the holotype was not investigated internally.

Coloration. Prior to preservation specimen uniformly light tan, flat with no iridescence; some longitudinal light-dark striations along tail. Snout tip and anterior margin of pre-orbital head a dark, sooty, gray-charcoal, with white marking around mouth, fairly uneven. Dorsal fin spine is a light to whitish color, dark brown along length of grooves of the anterior keel. Fins light gray-tan with a strong wash of light lavender, very anterior margins lighter in color, posterior margins frayed and lacking lavender 
coloration. Pores present on head along canals, light in color with dark boarders, varying in size. Lateral line canals of head and body darkly shaded. Tooth plates light yellow in color. Preserved specimen maintains similar body color, but lavender hue of fins is dulled.

Etymology. The Latin name is dedicated to Dr. Dominique A. Didier for her outstanding contributions to the systematics of this group of fish. Vernacular: the Falkor Chimaera, derived from the Japanese, fukuryu for "lucky dragon," and so named for this species uniquely pale, slender body resembling a famous description of luck dragons.

Distribution. A single specimen was collected from a seamount on the southern part of the Madagascar Ridge, located 3430'S, 4310’E near Walters Shoal in the Southwestern Indian Ocean. This was the only species of Chimaera encountered on this particular seamount (Figure 17). 


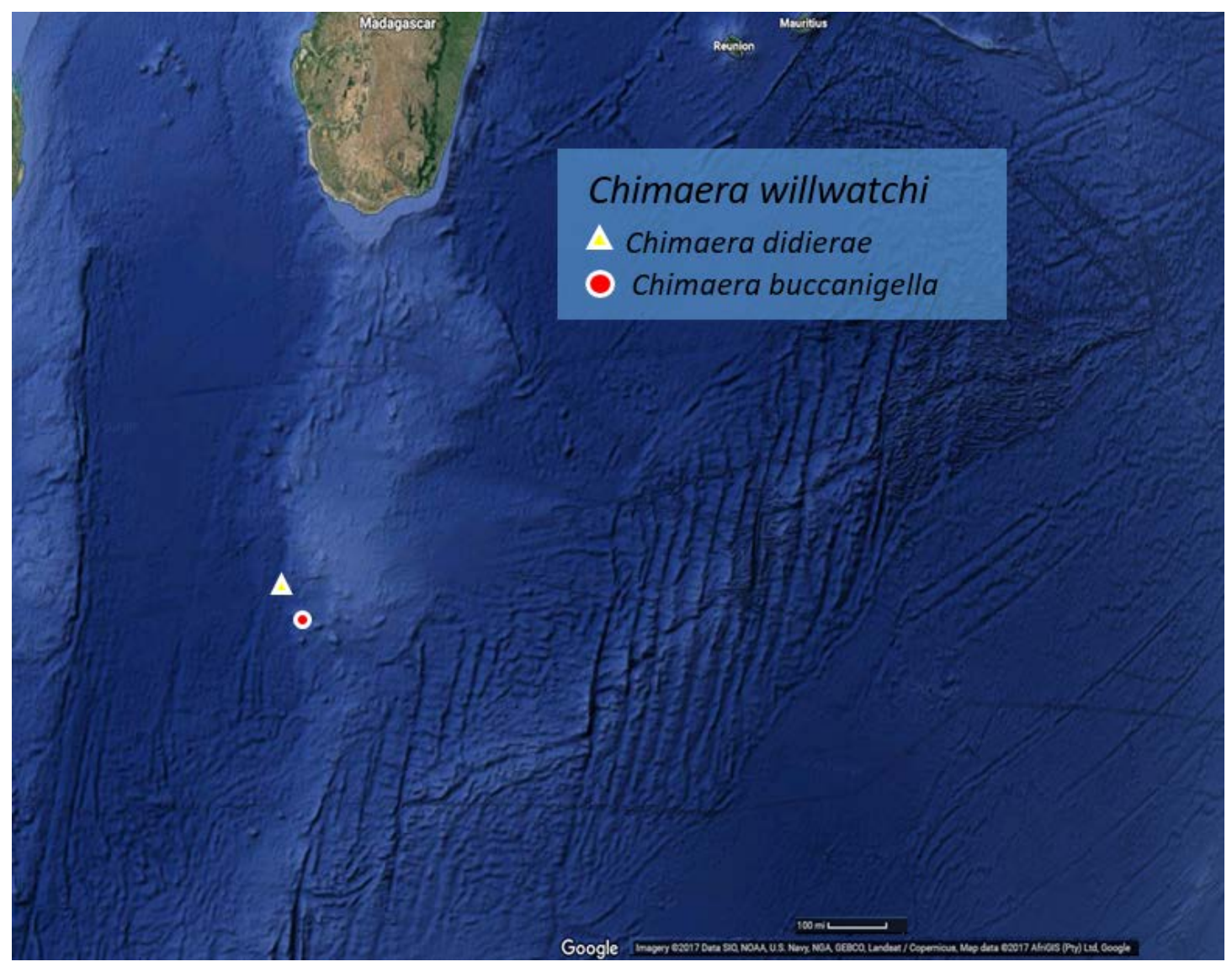

Figure 17. Map of study area denoting collection sites of Chimaera didierae, sp. nov., (yellow triangle), and Chimaera buccanigella, sp. nov. (red circle). Map data: Google, Image (c) 2017 DigitalGlobe.

Biological Notes. The female holotype was externally assessed to be in the early stages of maturity based on the presence of a developing, fleshy postanal pad used during copulation. In order to preserve its integrity, the unique type specimen was not examined internally. This species has a recorded depth range between $1000 \mathrm{~m}-1100 \mathrm{~m}$. Walters Shoal is the shallowest feature of the Madagascar Ridge and is relatively flat, providing a simple habitat structure. 
Comparison. Chimaera didierae is the third species of the genus known from the Southwestern Indian Ocean (Ebert, 2014; Eschmeyer, 2014) and can be distinguished from all other Chimaera species by the following combination of characters: light tan body color, without silver sheen, no defined patterning, spots, reticulations, or mottling; slender body, short trunk, long tail, relatively robust spine, very small unpaired fins, extremely deciduous skin.

Chimaera didierae is uniformly colored and lacks strongly defined spots, reticulations, or patterning, easily separating it from C. monstrosa, C. owstoni and $C$. panthera that depending on the species may have distinct spot patterns, usually brownish in color, mottling and or reticulations (Tanaka, 1905; Didier, 1998; Didier, et al., 2012; Ebert et al., 2013; Kemper et al., 2015). Chimaera didierae also lacks ridescent sheen or silvery body coloration usually found in C. argiloba, C. cubana, C. fulva, and C. phantasma (Jordan and Snyder, 1900; Didier et al., 2002, 2012).

Chimaera didierae is a medium-bodied species with a head of moderate length and height relative to body, without well-defined suborbital ridge, large eyes; thick, but moderately long dorsal spine equal to or subequal to height of first dorsal fin; relatively small paired fins. This combination of characters separates $C$. didierae from $C$. macrospina, which has a longer dorsal fin spine, $23.0 \%$ (25.3\% - 31.2\%) BDL vs $21.4 \%$ BDL, which exceeds first dorsal fin apex; larger fins, pectoral anterior margin longer, 39.5\% (37.5\% - 41.4\%) BDL vs 34.0\% BDL, greater second dorsal fin anterior height, $5.2 \%(4.5 \%-6.2 \%) \mathrm{BDL}$ vs $2.8 \% \mathrm{BDL}$; and smaller eye length to head length ratio, onefourth (25\%) head length vs one-third (34\%) head length. Chimaera notafricana is 
distinct in having a less robust body with tail tapering rapidly after pelvic girdle; pectoral fin anterior margin about 1.8 times head length vs 1.5 times head length; dorsal spine more strongly curved, exceeding apex of first dorsal fin; shorter eye length, $6.3-6.5 \%$ BDL vs. 7.9\% BDL. Chimaera lignaria is distinguishable by its larger, bulkier body and massively blocky head with bunt, squared snout vs slender body and moderately sized head and short thin snout; second dorsal fin taller, anterior height 4\% (4-7\%) BDL vs 2.7\% BDL, rubbery in texture and not easily split vs fins which are feathery, jagged, and easily frayed; pectoral fin rounded, not pointed at leading edge vs tear-drop in shape tapering to a point distally. Chimaera orientalis is separable by its shorter trunk length, 37.0\% (35.1-35.4\%) BDL vs 40.2\% BDL, longer dorsal spine 31.0\% (28.4\%) BDL vs $21.6 \%$, overlapping its much taller first dorsal fin, height $26.7 \%(22.8-25.0 \%)$ BDL vs 12.1\% BDL. Chimaera jordani is distinguished from C. didierae by its shorter trunk length, 52.8\% BDL vs 59.4\% BDL, shorter snout length, 2.6\% BDL vs 10.3\% BDL, smaller eye length, 6.6\% BDL vs 8.3\% BDL, and larger spine length, 26.6\% BDL vs 21.6\%, overlapping apex of first dorsal fin. Chimaera bahamaensis is distinguishable by its shorter trunk length, 35.0\% BDL vs 40.2\% BDL, much more pronounced snout, preoral length $12.0 \%$ BDL vs 9.0\% BDL, prenarial length $15.4 \%$ BDL vs $13.6 \%$ BDL; shorter eye length, 6.9\% BDL vs 8.3\% BDL, and eye height 3.5\% BDL vs 6.0\% BDL. Chimaera carophila is differentiable from C. didierae in having a longer preoral length, 11-19\% BDL vs 9.0\% BDL, smaller dorsal spine length, $18-20 \%$ BDL vs $21.6 \%$ BDL, which exceeds apex of first dorsal fin. 
The species most morphologically similar to $C$. didierae are C. obscura and $C$. opalescens, all characterized by a slender head, smaller fins, and spine not exceeding first dorsal fin. Chimaera obscura differs from C. didierae in having a longer dorsal spine, 27.2\% BDL vs 21.6\% BDL, larger fins, first dorsal fin height $23.0 \%$ (23.8\%) BDL vs 12.1\% BDL, second dorsal fin anterior height 5.0\% (4.9\%) vs 2.7\% BDL, pectoral fin anterior length 38.9\% (39.6\%) BDL vs 33.7\% BDL; and shorter eye length, 7.3\% (6.1\%) BDL vs $8.3 \%$ BDL. Chimaera opalescens is most similar to C. didierae, but is known from the northeastern Atlantic along the British Isles, France, and Greenland. It is similar to $C$. didierae in color being beige to tan, but is iridescent before preservation. Morphologically, C. opalescens tapers slower after its pelvic girdle into its tail transitioning into a greater tail height, $17.0-17.3 \% \mathrm{BDL}$ vs $12.4 \% \mathrm{BDL}$; shorter snout length, $4.1-6.2 \% \mathrm{BDL}$ vs $10.3 \% \mathrm{BDL}$, and shorter preoral length, $5.3-6.0 \% \mathrm{BDL}$ vs 9.0\% BDL; dorsal spine more curved with thicker ridge to origin, $4.9-5.9 \% \mathrm{BDL}$ vs $2.8 \%$ BDL, first dorsal fin taller, $11.9-17.1 \%$ vs $12.1 \%$ BDL, second dorsal fin with taller mid dorsal fin height, $3.5-4.4 \% \mathrm{BDL}$ vs $2.1 \% \mathrm{BDL}$; lateral line canals on side of head much longer, oronasal to nasal canal, 5.6\% BDL vs $2.3 \% \mathrm{BDL}$, length of the rostral canal $5.4 \% \mathrm{BDL}$ vs $1.8 \% \mathrm{BDL}$, length across nasal canal, $12.6 \% \mathrm{BDL}$ vs $4.9 \% \mathrm{BDL}$, infraorbital to angular canal $14.4 \%$ BDL vs $3.1 \%$ BDL; lateral line canals on dorsal portion of head much shorter, preopercular to main trunk, 2.1\% BDL vs 7.9\% BDL, orbital canal length $3.9 \%$ BDL vs $4.4 \%$ BDL, supratemporal canal length, $1.1 \%$ BDL vs 4.8\% BDL, and spine to supratemporal canal, $1.5 \% \mathrm{BDL}$ vs 3.3\% BDL. 


\section{Chimaera buccanigella, sp. nov., Dark-mouth chimaera}

Holotype. CAS 242335, 765 mm TL, 401 mm BDL, female, Southwestern Indian Ocean, Walters Shoal, $33^{\circ} 49^{\prime}$ S, $42^{\circ} 22^{\prime}$ E, bottom trawl between $495-960$ m, collector P.J. Clerkin, 3 March 2012.

Non-type. - 2 male specimens - MNHN 2004-0819 (BPS0693), 729 mm TL, 346 mm BDL, immature male, Madagascar Ridge, Southwestern Indian Ocean, 33²1'S $44^{\circ} 37^{\prime} \mathrm{E}$ to $33^{\circ} 28,317^{\prime} \mathrm{S}-44^{\circ} 50,525^{\prime} \mathrm{E}, 890 \mathrm{~m}-910 \mathrm{~m}$ depth, collected by the F/V Kerguelen de Tremarec, 31 July 2002; MNHN 2004-0818(BPS0692), 861 mm TL, 338 mm BDL, immature male, Madagascar Ridge, Southwestern Indian Ocean, 33²1’S $44^{\circ} 37^{\prime} \mathrm{E}$ to $33^{\circ} 28^{\prime} \mathrm{S}-44^{\circ} 50^{\prime} \mathrm{E}, 890 \mathrm{~m}$ - $910 \mathrm{~m}$ depth, collected by the F/V Kerguelen de Tremarec, 31 July 2002.

Diagnosis. Chimaera buccanigella, sp. nov. is distinguishable from other members of the genus by the following combination of characters: medium-sized species with moderate head length, $28.3 \% \mathrm{BDL}$, eyes very large, 9.6\% BDL, with defined suborbital ridge, relatively long snout measuring $13.1 \% \mathrm{BDL}$; trunk tapering rapidly into long tail, 60.4\% BDL; uniform light tan color, not iridescent, no defined patterning, spots, reticulations, or mottling, dark sooty brown on snout, dark markings in and around mouth (Figure 18c); pectoral fins with short anterior margin, 36.6\% BDL, pelvic fins broad, pointed distally, tear-drop shaped, with short anterior margin, $22.1 \% \mathrm{BDL}$; dorsal spine long, thin, and very straight, height of spine not exceeding height of first dorsal fin, when depressed just reaches origin of second dorsal fin; second dorsal fin not undulating, fins feathery, jagged, and easily frayed. Structure of the NADH2 gene. Chimaera 
buccanigella, sp. nov. is distinguishable from its closest congeners, Chimaera obscura Didier et al. 2008, and Chimaera opalescens Luchetti et al. 2001, by a combination of characters: small dorsal spine, generally small fins, small first dorsal fin height, small second dorsal fin anterior height, moderately large eyes, generally elongate head, body, and snout, and coloration.

Description. Morphometric proportions of the holotype and two paratypes are presented in Table 7. A medium-bodied species, head relatively large, head length $28.3 \%$ BDL, and thick, head width at suborbital ridge 14.4\% BDL, and about half (51.5\%) head length, suborbital ridge prominent and well-defined; snout length moderately long, 13.1\% BDL making up almost one-half (46.2\%) head length; snout width at base small, $7 \%$ head length. Trunk slightly compressed, length moderately short, 46.2\% BDL, height $24.5 \%$ BDL, abdomen height $24.7 \%$ BDL, tapers rapidly into long, whip-like tail, tail height 14.6\% BDL, tail length $60.5 \%$ BDL, with short caudal filament. Eyes large, length $9.6 \%$ BDL, about one-third (34.0\%) head length, and ovoid, located in posterior half of head, pre-orbital length $52.2 \%$ head length. Interdorsal space small, $2.7 \%$ BDL, pelvic-anal space large, 45.8\% BDL. Pectoral-pelvic space 34.8\% BDL, 1.2 times head length, and is shorter than pelvic caudal space. Pelvic-caudal space 46.6\% BDL, 1.6 times head length, and is shorter than snout-vent length 67.3\% BDL, 2.4 times head length. Skin smooth without denticles and not deciduous.

Pectoral fins relatively broad, pectoral fin width $18.5 \%$ BDL, and long, anterior margin 36.6\% BDL (1.3 times head length), triangular in shape, rounded near base; anterior margin increasing curvature distally to an acute apex, posterior margin slightly 
sigmoidal; pectoral fin base fleshy, asymmetrical in shape. Pectoral fins, when depressed posteriorly against the body, barely reach the origin of pelvic fins. Pelvic fins about half (60\%) size of pectoral fins, smaller than head length (78.0\% head length), anterior and posterior margins fairly straight, rounding sharply into base, tear-drop in shape, tapering to a point distally; fleshy base thick and oval in shape.
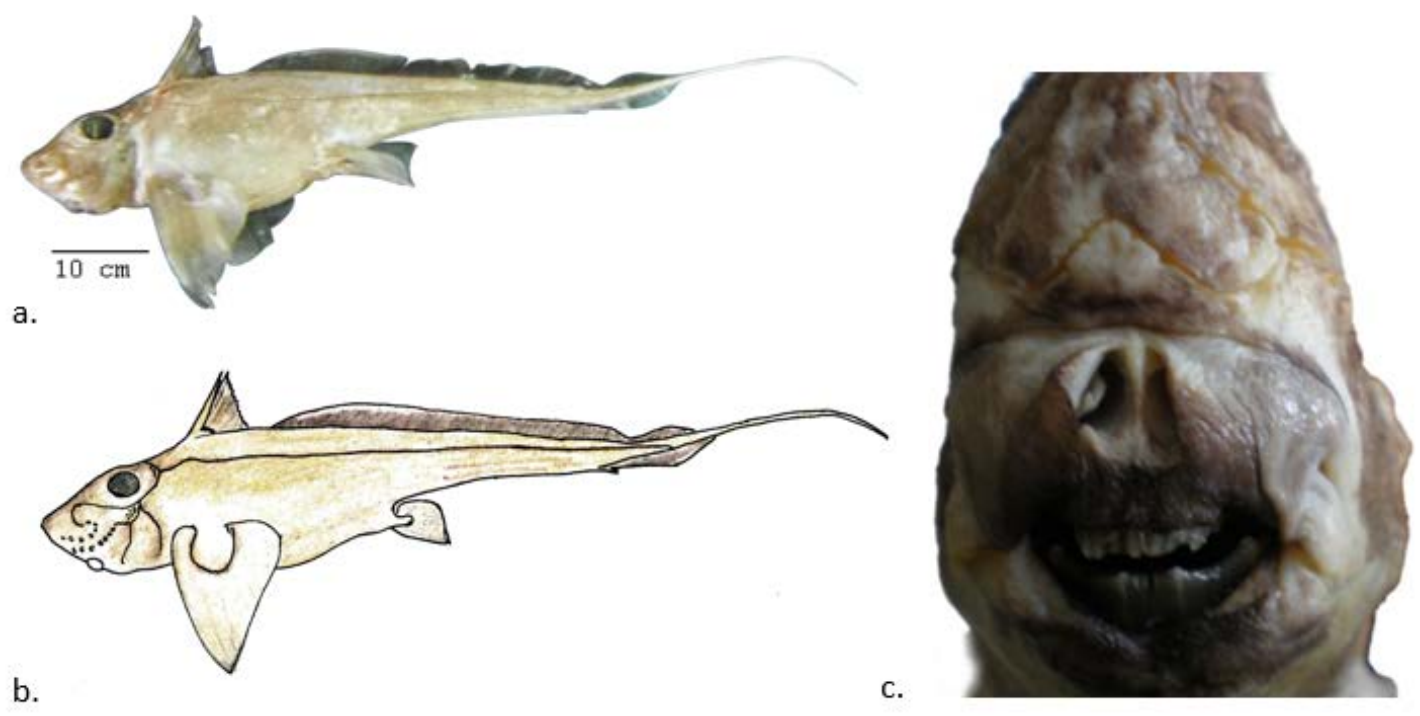

Figure 18. Full body lateral photograph of Chimaera buccanigella, sp. nov., a) holotype CAS 242335, immature female, $830 \mathrm{~mm}$ TL, $397 \mathrm{~mm}$ BDL b) illustration of holotype c) anterior view of holotype highlighting dusky mouth. Photos and illustration by P.J. Clerkin.

First dorsal fin small, height 15.5\% BDL, base 16.1\% BDL, triangular in shape; proceeded by moderately long (23.4\% BDL), thin (3.4\% BDL) spine; dorsal spine straight, triangular in cross section, keel strongly trenchant along anterior edge, and two closely spaced columns of serrations along distal half of posterolateral edges; spine length not reaching height of first dorsal fin, but reaching origin of second dorsal fin when depressed against the body. Second dorsal fin about one-fifth (22.2\%) height of first dorsal fin, elongate, $81.9 \% \mathrm{BDL}$, moderate in height and fairly straight, second 
dorsal fin anterior height 3.4\% BDL, second dorsal fin posterior height 3.5\% BDL, second dorsal fin mid height 3.5\% BDL. Caudal fin small, dorsal caudal margin 20.1\% BDL, ventral caudal margin 28.2\% BDL, small dorsal caudal height 2.4\% BDL, ventral caudal height $2.6 \%$ BDL, and symmetrical in shape; caudal fin tapers off very gradually and ends in a thin, short filament. Anal fin very thin and small. Edges of all fins fairly straight, feathery, jagged, and easily frayed.

Lateral line canals on head open, narrow grooves, those on snout with dilations fairly consistent in size and spacing (Table 8). Preopercular and oral lateral line canals share a common branch connecting to the infraorbital canal. Anterior origin of trunk lateral lines branches from junction of occipital and optic canals. Lateral line dips sharply ventrally then dorsally in a sigmoidal curve before returning to a relatively stable line at origin of dorsal spine and continuing posteriorly relatively non-undulating. Occipital canal short, 5.3\% BDL, directed semi-vertically to where it joins supratemporal and supraorbital canals, supratemporal canal short, 5.1\% BDL, and strongly curved. Supraorbital canal extending anteriorly from supratemporal junction, roughly sloping around dorsal eye margin. Infraorbital to angular canal relatively long, 8.9\% BDL, extending anteroventrally into mandibular and angular canals posterior of mid-eye. Angular canal horizontal before sensory pores and then continues anteroventrally. 


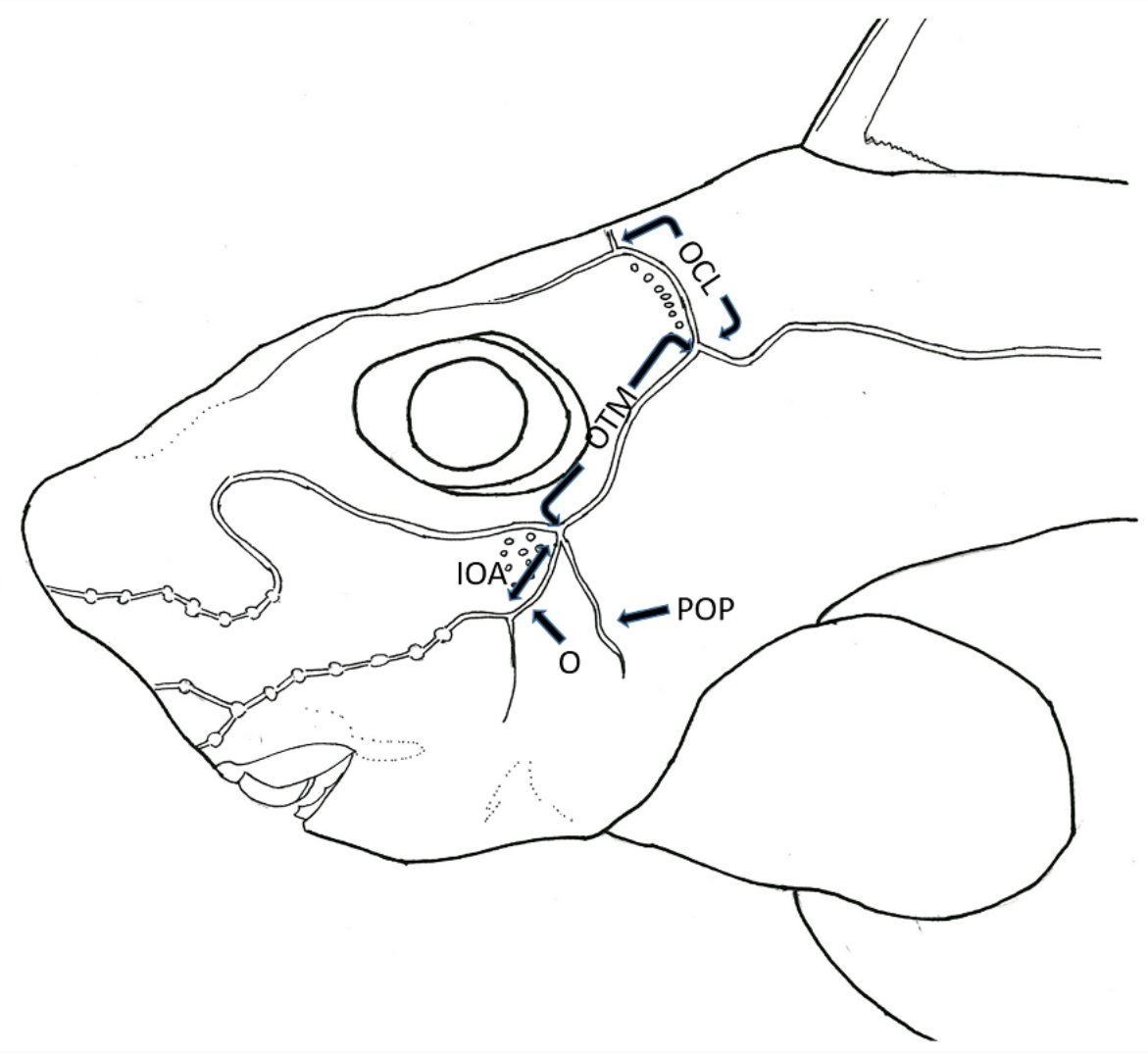

Figure 19. Illustration of Chimaera buccanigella, sp. nov., lateral lines of head showing: infraorbital to angular canal (IOA), junction of the oral-infraorbital canal to junction of the oral and angular canal, preopercular. Illustration by P.J. Clerkin.

Dentition. Tooth plates are smoky-gray in color, and lower tooth plates appear to lack visible rods. The type specimens were not dissected for detailed internal examination.

Coloration. Prior to preservation specimens uniformly light tan, flat with no iridescence; some longitudinal light-dark striations along tail. Dark, gray-brown on tip of snout, and dark marking directly around mouth with light blotted labials. Lines of head and body darkly shaded. Dorsal fin spine light white in color, dark brown along length of grooves of the anterior keel. Unpaired fins smoky black-gray in color, with white margin on anterior half of second dorsal fin. Pectoral and pelvic fins light blue with many brown 
speckles. Pores present on head along canals, light in color. Tooth plates dark smokygray in color. Specimens after preservation mostly retain body coloration.

Etymology. The Latin names bucca and nigella means respectively "mouth" and "dark," referring to the characteristic coloration of this species. The vernacular name, dark-mouth Chimaera, is based on the consistent dark coloration of this species' mouth.

Size. The two immature males measured $729 \mathrm{~mm}$ TL, $346 \mathrm{~mm}$ BDL, and $861 \mathrm{~mm}$ TL, $338 \mathrm{~mm}$ BDL, and the immature female measured $401 \mathrm{~mm}$ BDL, $765 \mathrm{~mm}$ TL.

Distribution. Known only from the deep waters of the Madagascar Ridge, in the Southwestern Indian Ocean $\left(33^{\circ} 21^{\prime} \mathrm{S}-42^{\circ} 22^{\prime} \mathrm{E}\right.$ to $\left.33^{\circ} 49^{\prime} \mathrm{S}-44^{\circ} 50^{\prime} \mathrm{E}\right)$ and southern part

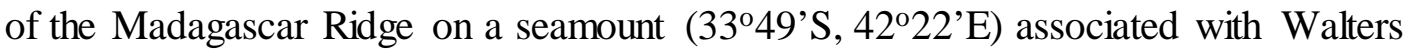
Shoal in the Southwestern Indian Ocean (Figure 17).

Biological Notes. Males were immature at $861 \mathrm{~mm}$ TL, $338 \mathrm{~mm}$ BDL. The female was externally assessed to be an immature based on the absence of a developing, fleshy postanal pad used during copulation. In order to preserve its integrity, the specimen was not examined internally. This species was recorded from a depth range of 495 m-960 m. Walters Shoal is the shallowest area of the Madagascar Ridge, which divides the Mozambique Basin and Madagascar Basin. Flat-topped seamounts and shallow plateaus characterized the area.

Comparison. Chimaera buccanigella is the fourth Chimaera species known from the Southwestern Indian Ocean (Ebert, 2014) and can be distinguished from other Chimaera species by the following combination of characters: light tan body color, without silver, 
no defined patterning, spots, reticulations, or mottling; stocky body, short trunk, tapering rapidly into a long tail; long, straight spine, skin not deciduous.

Chimaera buccanigella is uniformly colored, lacking any distinct patterning of spots, mottling or reticulations, such as found on C.monstrosa, C. owstoni and C. panthera that, depending on the species may have distinct spot patterns, usually brownish in color, mottling and or reticulations (Tanaka, 1905; Didier, 1998; Didier, et al., 2012; Ebert et al., 2013; Kemper et al., 2015). Furthermore, C. buccanigella lacks an iridescent sheen or silvery pink, grayish, or pale brown body coloration, some with faint stripes, usually found in C. argiloba, C. cubana, C. fulva, and C. phantasma (Jordan and Snyder, 1900; Didier et al., 2002, 2012).

Chimaera buccanigella is a medium-sized species with relatively long, conical snout, moderately sized head, defined suborbital ridge, large eyes, moderately long trunk length tapering rapidly into long tail, with long spine, very straight and not exceeding the height of first dorsal fin. This combination of characters separates $C$. buccanigella from $C$. macrospina, which has a shorter snout to vent length, 58.7\% (55.1-61.3\%) BDL vs 67.3\% BDL, short trunk length, 39.8\% (37.3-40.5\%) BDL vs 46.2\% BDL, very weak suborbital ridge vs well defined, shorter eye length, 6.6\% (5.7-8.3\%) BDL vs 9.6\% BDL, greatly exceeding apex of taller first dorsal fin, 19.7\% (19.4-24.4\%) BDL vs $15.5 \%$ BDL. Chimaera notafricana is distinct from C. buccanigella in having a dorsal spine more strongly curved and shorter in length, 22.1\% (15.9-18.5\%) BDL vs 23.4\% BDL, and smaller eye length $6.3 \%-6.5 \%$ BDL vs. 9.6\% BDL. Chimaera lignaria is distinguishable from C. buccanigella by its larger body, bulkier head, and squared snout. 
Chimaera orientalis has a much longer spine, 31.0\% (28.4\%) BDL vs 23.4\% BDL, which exceeds the apex of a taller first dorsal fin, height $26.7 \%$ (22.8-25.0\%) BDL vs $15.5 \%$ BDL. Chimaera jordani is distinguished from C. buccanigella by its shorter snout length 2.6\% BDL vs $13.1 \%$ BDL, smaller eye length $6.6 \%$ BDL vs $9.6 \%$ BDL, and larger spine length, $26.6 \%$ BDL vs $23.4 \%$, which overlaps apex of first dorsal fin. Chimaera bahamaensis is distinguishable by its smaller trunk length, $35.0 \% \mathrm{BDL}$ vs $42.2 \% \mathrm{BDL}$, smaller eye length $6.9 \%$ BDL vs 9.6\% BDL, and dorsal spine exceeding apex of first dorsal fin. Chimaera carophila is differentiable from C. buccanigella in having a smaller head length, $22-24 \%$ BDL vs $28.3 \%$ BDL, shorter eye length, $8 \%$ BDL vs $9.6 \% \mathrm{BDL}$, shorter dorsal spine length, $18-20 \%$ BDL vs $23.4 \%$ BDL, spine usually exceeding apex of first dorsal fin.

The species most similar to C. buccanigella, with a conical snout, defined suborbital ridge, large eyes, and long spine not exceeding first dorsal fin, are $C$. obscura and $C$. opalescens. Chimaera obscura differs from C. buccanigella in having a longer dorsal spine, 27.2\% BDL vs 23.4\% BDL; larger fins, first dorsal fin 23.0\% (23.8\%) BDL vs 15.5\% BDL, second dorsal fin anterior height 5.0\% (4.9\%) vs 3.4\% BDL; and smaller eye length $7.3 \%$ (6.1\%) BDL vs 9.6\% BDL. Chimaera opalescens is similar to $C$. buccanigella in color being beige to tan, but is iridescent before preservation; all around less elongate, trunk length $33.7-41.1 \% \mathrm{BDL}$ vs $46.2 \% \mathrm{BDL}$, head length $20.1-23.8 \%$ BDL vs $28.3 \%$ BDL; features of head less elongate with shorter pre-oral length, 8.1-11.5\% BDL vs $14.8 \% \mathrm{BDL}$, prenarial length $2.8-4.1 \% \mathrm{BDL}$ vs $9.1 \% \mathrm{BDL}$, snout 
length $4.1-6.3 \%$ BDL vs $13.1 \%$ BDL; spine not as robust with ridge to origin $4.9-5.9 \%$ BDL vs 3.1\% BDL.

\section{Comparison of New Southwestern Indian Ocean Chimaera Species}

The three new Chimaera species can be separated from each other by a combination of external characteristics. Chimaera willwatchi is large-bodied and distinct in its darker, heavily mottled body coloration, and white fin margins. Chimaera willwatchi is a more robust species, distinguishable from $C$. didierae by its larger, blockier head and trunk, squared snout; larger paired fins, pectoral fin width 22.1\% (19.6-23.2\%) BDL vs $16.7 \%$ BDL, pelvic fin anterior margin 25.0\% (22.9-26.8\%) BDL vs 19.9\% BDL; dorsal fin spine longer, 27.3\% (22.9-24.9\%) BDL vs 21.6\% BDL, exceeding apex of first dorsal fin, longer first dorsal fin base length 17.6\% 14.2-17.9\%) BDL vs 13.2\% BDL, first dorsal fin height 20.3\% (16.2-19.1\%) BDL vs 12.1\% BDL, second dorsal fin taller anterior margin, $6.6 \%(3.8-7.2 \%)$ BDL vs $2.7 \%$ BDL.

Chimaera willwatchi can be distinguished from C. buccanigella by its blockier body shape, and by a shorter trunk length, 43.1\% (36.4-44.4\%) BDL vs 46.2\% BDL; taller head height, $26.6 \%(23.0-26.7 \%)$ BDL vs $21.4 \%$ BDL; exceeding apex of first dorsal fin, first dorsal fin taller, 20.3\% (16.2-19.1\%) BDL vs 15.5\% BDL, second dorsal fin with taller anterior margin, $6.6 \%(3.8-7.2 \%)$ BDL vs $3.4 \%$ BDL, but not as long $74.8 \%$ (70.1-77.5\%) BDL vs $81.8 \%$ BDL.

Morphometrically, C. buccanigella and C. didierae are the closest congers with both species being light tan in color with dark snouts, blue or purplish fins, and proportionally smaller unpaired fins. However, C. didierae is distinguishable by its shorter snout to vent 
length, 59.4\% BDL vs 67.3\% BDL, while having a longer tail length 63.7\% BDL vs 60.5\% BDL; more slender overall with snout height 7.5\% BDL vs 9.5\% BDL; shorter snout, $10.3 \%$ BDL vs 13.1\% BDL, smaller eyes 8.3\% BDL vs 9.6\% BDL, less blocky head with less defined suborbital ridge, head width at suborbital ridge $12.0 \%$ BDL vs 14.5\% BDL; fins smaller overall, pectoral fin anterior margin more strongly curved and shorter, 33.7\% BDL vs 36.6\% BDL, pelvic fin anterior margin 19.9\% BDL vs $22.1 \%$ BDL, first dorsal fin height 12.1\% BDL vs 15.5\% BDL, first dorsal fin base 13.2\% BDL vs $16.2 \%$ BDL, second dorsal fin base $77.1 \%$ BDL vs $81.9 \%$ BDL, second dorsal fin anterior margin 2.7\% BDL vs 3.4\% BDL, ventral caudal height 1.1\% BDL vs 2.6\% BDL.

The maximum likelihood tree topology at the NADH2 locus for SWIO chimaeras indicates that $C$. willwatchi, $C$. didierae and $C$. buccanigella form three distinct lineages, different from morphologically similar Chimaera species, as well as known South African species (i.e. C. notafricana, H. africanus) (Figure 13). Chimaera buccanigella is clearly distinct from all species incorporated in this analysis, including the morphologically similar C. opalescens and C. obscura, with $100 \%$ bootstrap support. Chimaera didierae also is distinguishable from the other species, recovered as a sister species to C. notafricana, known from South Africa, in this analysis. The morphologically similar C. opalescens, C. obscura, and C. buccanigella are clearly also distinct from $C$. didierae based on the NADH2 molecular data. Chimaera willwatchi is recovered as a single, distinct species (100\% bootstrap support), with 4 sub-clades. These sub-clades are based on the location of collection within the SWIO. There are two clades of SWIO Ridge individuals, however, this encompasses a very large area. All but 
one of the individuals (KX761206) in the SWIO Ridge clade, sister to the Walters Shoal clade, with detailed locality information, was collected from the western region of the SWIO Ridge (see map, Figure 9). The other SWIO Ridge clade individuals were all collected from a more eastern region of the SWIO Ridge. The individual C. willwatchi, KX761206, was collected from the eastern region of the SWIO Ridge. The exact location of two individuals that fall within these sub-clades, KX761197 and KX761216, were not recorded, but are known to be within the SWIO. The NADH2 locus suggests that $C$. willwatchi, C. didierae, and C. buccanigella are distinct species from other morphologically similar species and those nearby in locality. However, we caution that this tree topology is based on only a single gene with limited species sampling, and may not be congruent with the true species tree based on multiple markers and denser taxon sampling.

\section{Discussion}

Chimaeroid identification can be difficult due to poor original descriptions, and maybe further complicated by the poor condition of many specimens (Kemper et al., 2015), small sample sizes, distortions and shrinkage of specimens during preservation, and variation and limitation of certain measurements used to describe species. There are also potential issues with sexual dimorphism, ontogenetic shifts with growth, color morphs and variation within species. As a result, the family Chimaeridae is one of the most poorly known groups of cartilaginous fish, with the International Union for Conservation of Nature (IUCN) assessing more than half of all known Chimaera and Hydrolagus species as data deficient (Dulvy et al., 2014). Consequently, this group is 
taxonomically problematic, and therefore effective conservation and ecosystem-based management requires accuracy and improvement of species-specific identification of regional species. As the foundation upon which biological sciences rest, accurate and universal taxonomy is essential to this management goal (Simpfendorfer et al., 2011).

\section{Key to Indian Ocean Chimaera Species}

The following key to Indian Ocean Chimaeras is a modification of Ebert (2014). This key includes the three new species described here. There are a few caveats relative to this key. Many Chimaera species share similar body coloration, but color can vary greatly within species. Therefore, the key groups these species based on the most consistent and reliable characteristics, including body coloration characters, such as silvery or not silvery, and the presence or absence of distinct patterning on the body. Branching of oral and preopercular canals is not included in the key since this characteristic was determined to be inconsistent within a species.

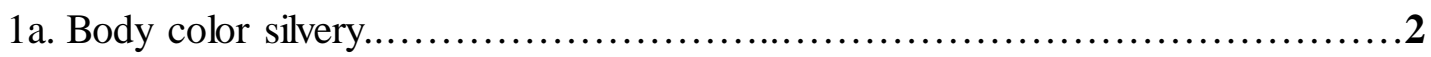

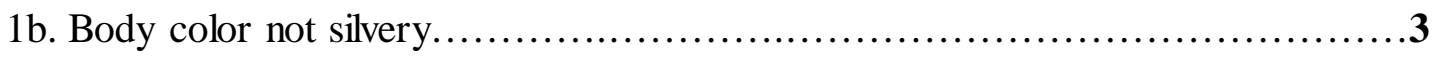

2a. Dorsal-fin spine exceeding apex of first dorsal fin; trunk lateral line with tight sinuous undulations or broad undulations anterior to pelvic fins; prepelvic tenaculum of males with 4-5 denticles, claspers of mature males exceeding free tips of pelvic fins.............................................maera argiloba

2b. Dorsal-fin spine not exceeding apex of first dorsal fin; trunk lateral line without 
sinuous undulations along its length; prepelvic tenaculum of males with 6-7 denticles, claspers of mature males not exceeding free tips of pelvic fins. Chimaera fulva

3a. Dorsal-fin spine small, not exceeding height of first dorsal fin, and fairly Straight.

3b. Dorsal-fin spine large, exceeding height of first dorsal fin, robust, slightly to

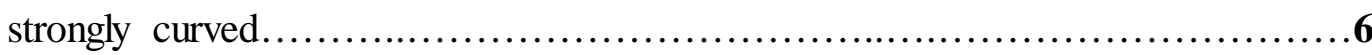

4a. Long slender body, conical snout; pelvic fins relatively small, and not distinctly

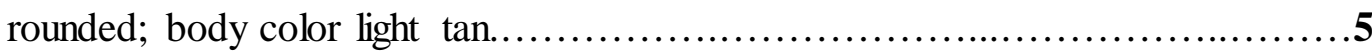

4b. Robust body, massive, blocky head, blunt squared snout; pelvic fins large and rounded; body color grey-blue, purple-brown or lavender.......Chimaera lignaria

5a. Suborbital ridge well defined, eyes very large; dorsal spine thin, and very straight, second dorsal fin moderately long, mouth distinctly dark, uniform light tan color, not iridescent . ..............................Chimaera buccanigella new species

5b. Very slender body, shorter snout, suborbital ridge poorly defined; eyes fairly large; dorsal spine robust, slightly curved; second dorsal fin very long; mouth with some dark marking, uniform light toffee-tan color, not iridescent Chimaera didierae new species 
6a. Medium to small sized head, conical snout, without well-defined suborbital

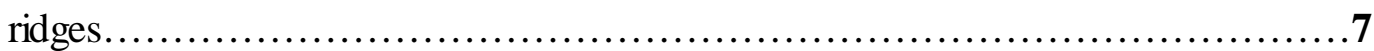

6b. Large, blocky head, well-defined suborbital ridge, blunt, squared snout; dorsal spine thick, slightly curved, exceeds the apex of the first dorsal fin; body color brown, marbled around snout; posterior margin of first dorsal fin often distinctly white Chimaera willwatchi newspecies

7a. Eyes moderate in size, greater than one-third head length, dorsal spine one-third to one fourth BDL; body color uniform dark brown or black........Chimaera macrospina 7b. Eyes small, less than one-third head length, dorsal spine one-fourth to oneseventh BDL; body color blackish-brown.......................Chimaera notafricana 


\section{Chapter Two: The Natural and Life Histories of Deep-sea Chondrichthyans in the Southwestern Indian Ocean}

\section{Introduction}

Approximately 3 billion people (40\% of the world's population) rely on wild-caught marine fish as their source of dietary protein (FAO, 2012). In meeting this global demand, increased commercial fishing efforts supported by advancements in technology have had a profound anthropogenic influence on natural marine environments (Jackson, 2010). Unfortunately, our understanding of this human impact on marine biodiversity and species populations is, for the most part, based primarily on limited information from retrospective studies that tend to focus on commercially valuable species or a handful of charismatic “megafauna” species (Collette et al., 2011; McClenachan et al., 2012; Ebert and Van Hees, 2015). Few studies have established a baseline of information for noncharismatic shark species, and even fewer have focused on sharks in the deep sea, where intrinsic physical obstacles and financial constraints make sampling especially challenging (Morato et al., 2006).

Despite harsh conditions, the deep sea is the largest habitable environment on the planet (Gage et al., 1991; Robinson, 2009) and serves as habitat for nearly half of all known shark species (Kyne and Simpfendorfer, 2010). Most deep-sea shark species, however, are very poorly known. A recent study by Dulvy et al., (2014) reviewed the IUCN Red List Assessments for chondrichthyans based on habitat, and found nearly onehalf were assessed as Data Deficient. Of those species accessed, 38.4\% of the 482 coastal and continental shelf species, $10.3 \%$ of the 39 neritic and epipelagic species, $50.0 \%$ of the 8 mesopelagic species, $54.5 \%$ of the 33 freshwater obligates, and $57.6 \%$ of the 479 deep- 
sea species were Data Deficient. Based on this study, it is apparent that those species occurring in the deep-sea are the least known group of cartilaginous fishes.

From the limited information available, researchers estimate that deep-sea sharks are less fecund and slower to reproduce than their coastal counterparts (Morato et al., 2006; Simpfendorfer and Kyne, 2009). As a result, deep-sea fisheries might need to adapt a different management approach that takes into account this lower productivity. Here we present natural history and life history information for 27 species of deep-sea sharks and four species of Holocephali encountered in the Southwestern Indian Ocean during two surveys of the Madagascar Ridge and the Southwest Indian Ocean Ridge.

\section{Materials and Methods}

Biological Data. The total length, sex, and maturity status were recorded for each specimen captured; maturity status assessment is detailed below. Standard measurements for sharks followed Compagno (1984) and Francis (2006a). Total length $\left(L_{T}\right)$ was recorded as the distance between the snout tip to the point on the horizontal axis intersecting a perpendicular, vertical line drawn down from the distal-posterior most point of the caudal lobe, while the precaudal length (PCL) was defined as the distance from the snout tip to the dorsal insertion of the caudal fin. All chimaerid species have a caudal fin that slowly tapers off into a long, whip-like filament (Didier et al., 2012). This distal extension of the caudal fin is often broken or absent, and because the filament is homogenous in form it is never truly possible to determine whether the caudal fin is completely intact. Total length measurements are, therefore, prone to error due to damage in chimaerids, and reproductive and maturity parameters are best expressed in terms body 
length (BDL): the distance from dorsal edge of gill opening to origin of dorsal margin of caudal fin (Inada and Garrick, 1979; Compagno et al., 1990; Hardy and Stehmann, 1990).

Maturity Determination. Shark maturity was assessed by external visual inspection of claspers in males and internal inspection of reproductive organs in females (Figure 20) following Ebert (1996, 2005) and Ebert et al. (2006). Males were considered mature when the claspers were elongated, extended beyond the posterior free margin of the pelvic fins, were firm, and had their terminal cartilage elements calcified. Adolescent males had elongated claspers surpassing the free rear tips of the pelvic fins, but claspers were flexible and lacked calcification. Juvenile males had short, flexible claspers not reaching past the posterior margin of the pelvic fins. Inner clasper length was measured from the apex of the cloaca to the distal tip of the clasper and the ratio of clasper length to $L_{\mathrm{T}}$ (thus normalizing clasper length) was plotted against $L_{\mathrm{T}}$. An abrupt change in the clasper length to $L_{\mathrm{T}}$ ratio has been used previously to indicate maturity (Ebert 2005). Males were not examined internally for maturity.

Females were considered mature when large yolky oocytes were present in the ovaries, and the oviducal gland was well developed, which was visually determined by a pendulous and distinctly differentiated from the uterus (Ebert, 1996). The uterus was enlarged with pendulously posterior portions hanging free from the body cavity (Figure 20-b). Adolescent females had small ovaries with some differentiation, but less developed, smaller oocytes lacking defined yellow yoke. The oviducal gland underdeveloped along a thin, constricted uterus closely attached to the body. Juvenile females lacked differentiation of oocytes and the oviducal gland was not differentiated 
from the thin uterus. Any individual (male or female) with a partially healed umbilical scar was considered a neonate (Carlson, 1999).
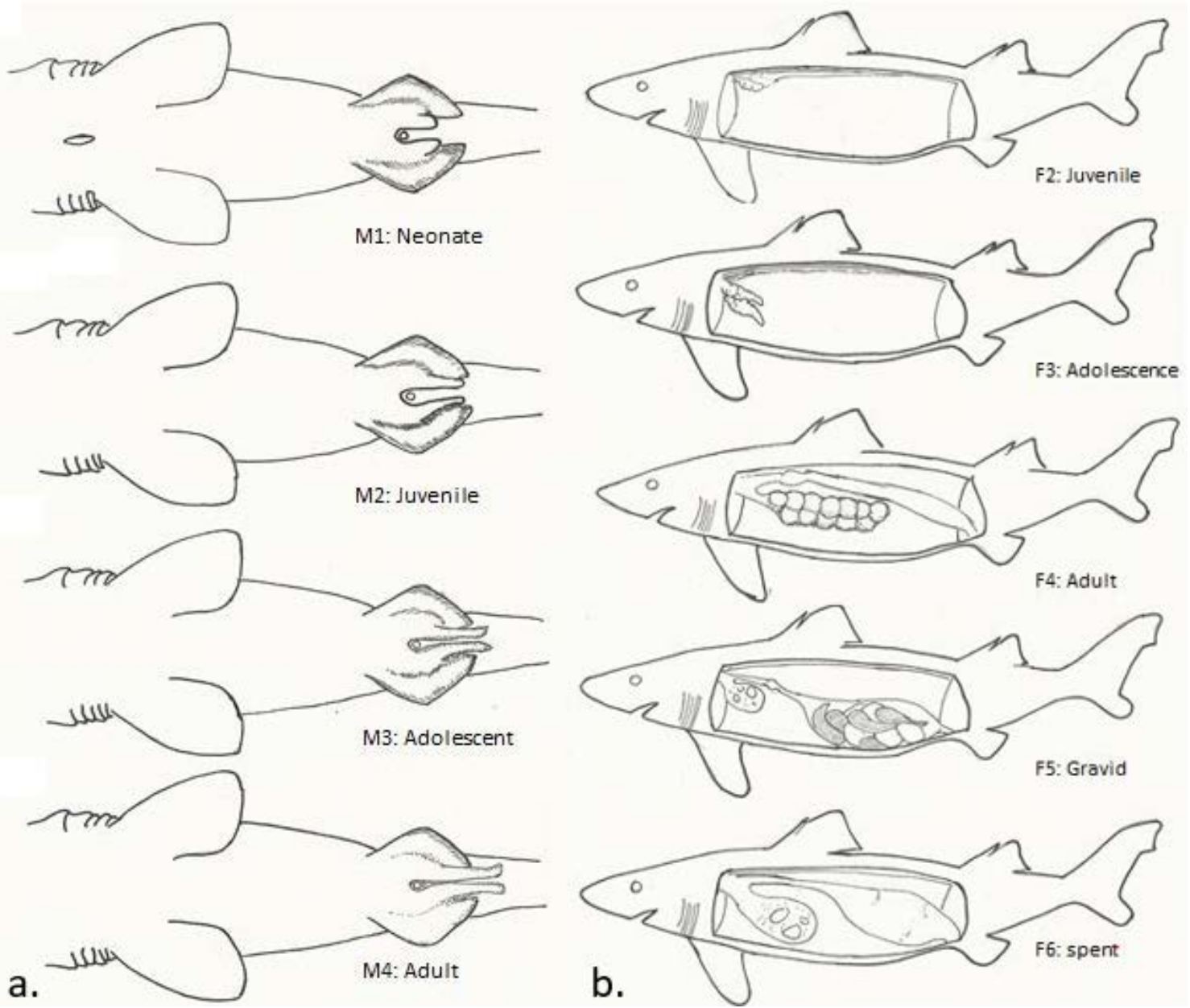

Figure 20. Illustration of maturity ranking system for sharks a) males, b) females. Illustration by P.J. Clerkin.

Number and size of oocytes and mature eggs were plotted against $L_{\mathrm{T}}$ to assess possible change in fecundity with increasing $L_{\mathrm{T}}$. The immature gamete formed in the ovary during oocytogenesis was considered an oocyte. This germ cell develops into a mature egg (ovum), during ovulation (release of the oocyte from the ovaries), which makes it available for fertilization by fusion with a male gamete cell (sperm) (William, et 
al., 2003). To simplify discussion, the term oocyte will be used to refer to egg cells found with the ovaries, while egg will be used to describe egg cells in the uterus.

Sex and length of pups were recorded and plotted against mother's $L_{\mathrm{T}}$ to determine the relationship of offspring sex ratios and size with mother's $L_{\mathrm{T}}$. Litter size was recorded and plotted against the mother's $L_{\mathrm{T}}$ to investigate the relationship between fecundity and increase in mother's $L_{\mathrm{T}}$. Width of the oviducal gland was measured at its widest distance across the gland and the ratio of oviducal gland to $L_{\mathrm{T}}$ was plotted against $L_{\mathrm{T}}$. An abrupt change in the oviducal gland width ratio indicated maturity (Ebert, 2005). To measure how fecundity changed with mothers' $L_{\mathrm{T}} \mathrm{S}$, oocytes were counted separately in left and right ovaries, and the largest oocyte from each side was measured using a sliding caliper. Oocyte count and size were compared between left and right ovaries using a paired t-test with the null hypothesis of no difference between the mean number of right and left oocytes ( $>>0.05)$ (Zar, 1996; Ebert, 2005).

Chimaeridae maturity was assessed modified from Didier and Rosenberger (2002) and Barnett et al. (2009), by external visual inspection of frontal tenaculum, prepelvic tenacula, and claspers in males, and external inspection of postanal pad and oviduct opening in females (Figure 21). Males were considered mature when secondary sexual characters were developed, with frontal tenaculum fully erupt and bearing thorn-like denticles, prepelvic tenacula able to articulate forward out of pockets, and claspers elongated, stiff and calcified with distal portions ending in fleshy tissue covered by a fine shagreen of denticles (Figure 21-a). Adolescent males were developing, with frontal tenaculum in the process of erupting from head, prepelvic tenacula developing in pockets, 
claspers beginning to elongate, but were still flexible and lacked calcification. Juvenile males were undeveloped with frontal tenaculum not erupted on head, but often marked with white outline, prepelvic tenacula small, undeveloped, and not articulating forward out of pockets, claspers present, but very small and flexible. Total clasper length was measured from the apex of the cloaca to the distal tip of the clasper, and plotted as a ratio of BDL. An abrupt change in the clasper length to BDL ratio indicated maturity (Barnett et al., 2009).

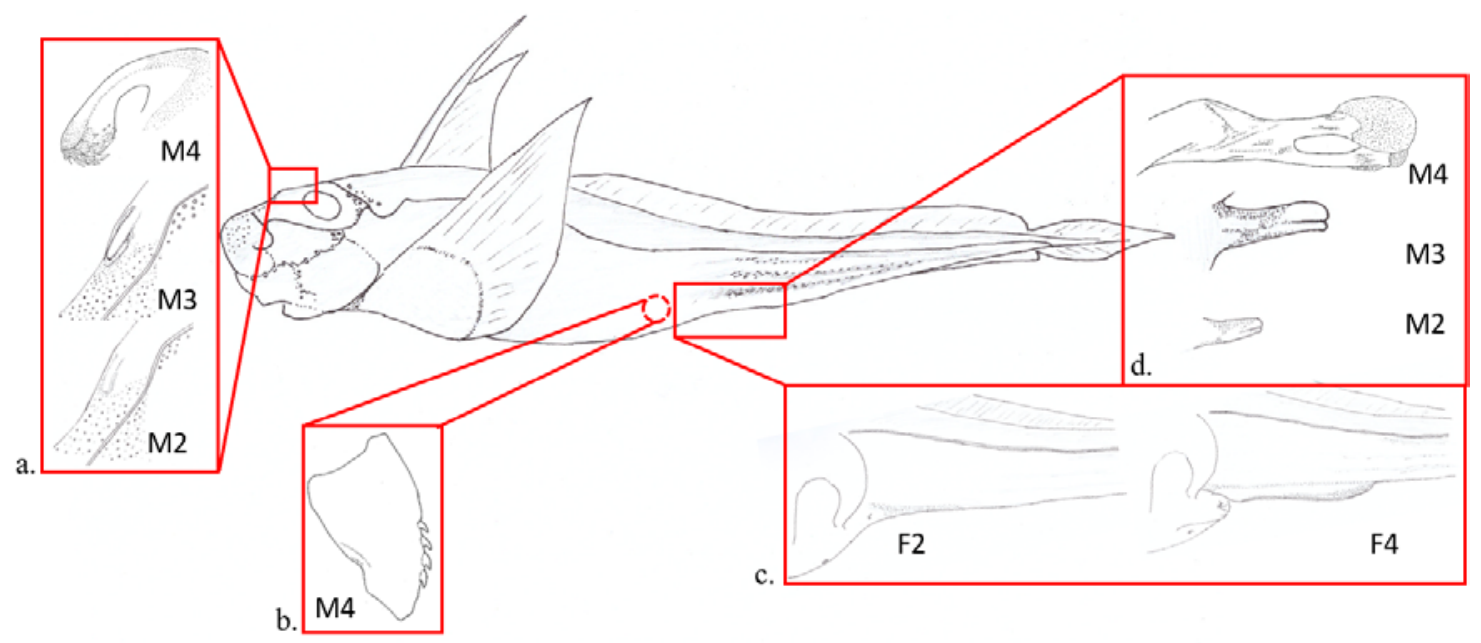

Figure 21. Illustration of maturity ranking system for chimaeroids showing stages of development of a) frontal tenaculum, b) pre-pelvic tenaculum, c) anal pad, and d), claspers. Illustration by P.J. Clerkin.

Females were considered mature when a large swollen postanal pad was well defined from tail musculature, and oviduct openings were large and dilated, often swollen and textured with papule (Figure 21-b). Adolescent females had less-developed postanal pads, differentiable from tail, but not yet well defined, and oviduct opening small or starting to dilate, but not swollen or textured. Juvenile females with postanal pad undeveloped, sometimes darker in color, but not swelling to the point of being differentiable from the tail, and the oviduct opening not dilated, without papule, and appear as deep dimples 
posterior to vent. Height and length of postanal pad were recorded and the ratio of postanal pad to BDL was plotted against BDL. An abrupt change in the postanal pad height and length to BDL ratio indicated maturity (D.A. Didier, Millersville University, pers. comm).

Sexual Dimorphism. Sexual dimorphism exists in Chondrichthyans in several forms, such as tooth shape, pelvic fin length, and presence of claspers (external copulatory appendages) in males (Ebert, 2005). This study will focus on total length (BDL in chimaerids) as a dimorphic character since it is easily quantifiable, and directly related to maturity (Hoenig, 1990). Maximum length and size at maturity were compared between the sexes of each species for which both males and females were encountered (Table 9).

Analysis. Length frequencies for males and females were plotted by $2 \mathrm{~cm}$ bins to illustrate size distribution. The overall proportions of each sex, as well as sex ratios of adults and sub-adults, were analyzed using a $\chi^{2}$ goodness of fit test to determine whether the observed ratios significantly deviated from unity and are presented in Table 10 (Zar, 1996). The theoretical lengths at which $50 \%$ of male and $50 \%$ female specimens were mature $\left(L_{T 50}\right)$ was estimated for both sexes of each species using a logistic regression in JMP (Roa et al., 1999; Mollet et al., 2000; Neer \& Cailliet 2001).

Egg Cases. Egg case measurements were taken following Ebert et al. (2006) and Ebert and Clerkin (2015). In addition to egg case length (ECL), nine measurements were taken, anterior border width (AWB), anterior respiratory fissure length (AFL), anterior width (AW), egg case height (HI), posterior border width (PBW), posterior respiratory 
fissure length (PFL), posterior width (PW), waist width (WW), and flange height (FH), and normalized as a percentage of ECL (Figure 22).

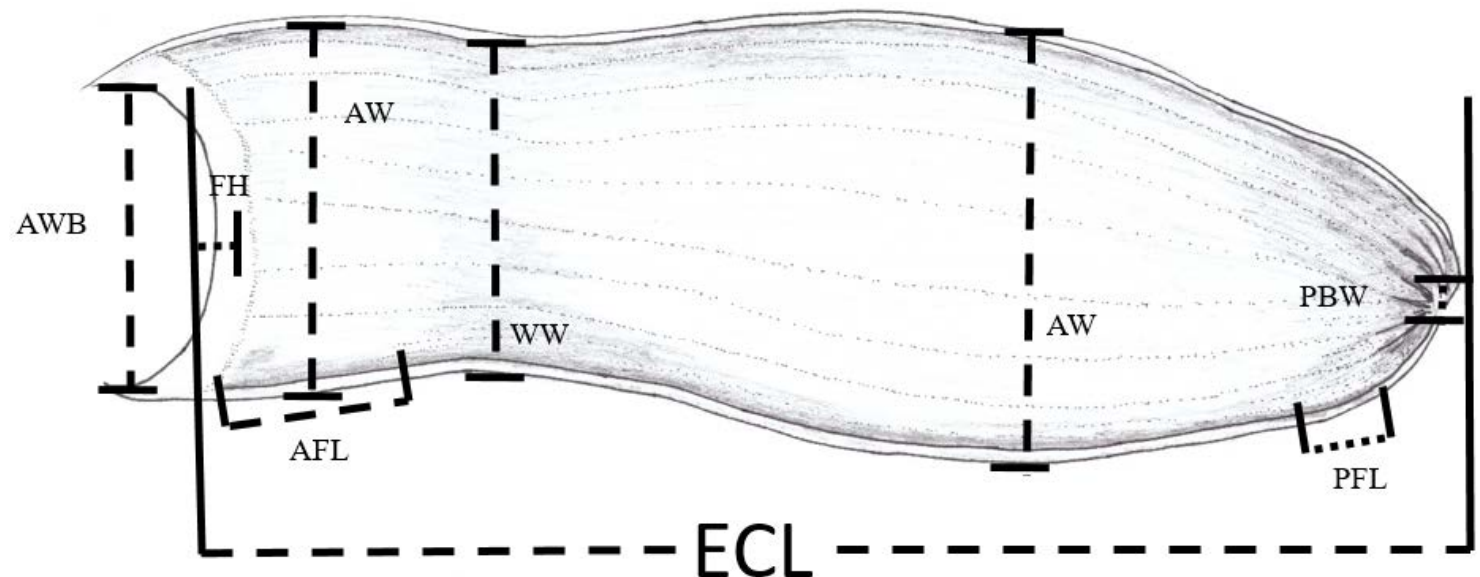

Figure 22. Diagram of egg case measurements: egg case length (ECL), anterior border width (AWB), anterior respiratory fissure length (AFL), anterior width (AW), egg case height $(\mathrm{HI})$, posterior border width (PBW), posterior respiratory fissure length (PFL), posterior width (PW), waist width (WW), and flange height (FH), and normalized as a percentage of ECL. Illustration by P.J. Clerkin.

Diet. Diet data were collected opportunistically at sea following Ebert et al. (1991). A total of 341 stomachs (8.1\% of the sharks of this survey) from 12 species were found to have prey. Stomach contents were removed and broadly categorized into five higher taxonomic groups: bony fish, shark, cephalopod, other invertebrate, or mammal. Percent volume was visually estimated for each diet category and item count recorded. The importance of each prey item was evaluated by a percent volume and frequency of occurrence.

Distribution. A total of 427 otter or bottom trawls were deployed with 216 tows (138 bottom tows, 78 mid-water tows) resulted in sharks captured as bycatch from 40 stations. Mesh size of the cod end was constant on all trawls, thus eliminating gear based sampling bias. 
The stations sampled were simplified into two major areas based on the distinct ecosystems of the region: Madagascar Ridge (114 tows) — including the northern region (7 tows) and Walters Shoal in the southern region (97 tows) — and the Southwest Indian Ocean Ridge (112 tows). Distribution and relative abundance were investigated for each region and expressed as a percent species composition, and a total number of species encountered only in that region. Furthermore, sex and maturity data is provided, where sufficient information was gathered, for each region to determine if intraspecific segregation behavior occurs. Species composition was examined using a non-metric multidimensional scaling analysis in PRIMER to explore how fauna relative abundance compares between ecosystems. SIMPER pairwise comparison was used to quantify contributing and cumulative Bray Curtis similarity (species contributions) percentages of species composition between regions. SIMPER pairwise comparison of contributing and cumulative Bray Curtis similarity (species contributions) between trawl gear types was used to investigate species location in the water column. The nMDS scores were plotted to illustrate dissimilarities of species composition between gear types.

\section{Results}

Centrophorus granulosus (Bloch \& Schneider, 1801), Gulper shark. In all, 34 C. granulosus (21 females and 13 males) were collected with an overall female to male (F:M) sex ratio of 1:0.6 (Table 9a), not significantly different from the expected 1:1 ratio ( $p>0.05$ ) (Table 10). However, sex ratios were significantly different when compared by maturity status, with an inverse ratio between adults being primarily male, 1:9 (pvalue $<0.05$ ), and juveniles dominated by females, 1:0.2 (p-value $<0.05$ ). 
Males ranged from 55 to $126.3 \mathrm{~cm} L_{\mathrm{T}}$, with 9 mature individuals (69.2\%), the smallest mature measuring $118.3 \mathrm{~cm} L_{\mathrm{T}}$ and the largest immature measuring $125.4 \mathrm{~cm} L_{\mathrm{T}}$ (Figure 23-a). Clasper length increased between 118 and $120 \mathrm{~cm} L_{\mathrm{T}}$ (Figure 23-b), with the smallest mature occurring at $93.7 \% L_{\mathrm{T}} \max$, and the $L_{\mathrm{T}} 50$ was estimated to be 117.5 $\mathrm{cm} L_{\mathrm{T}}$. 
Table 9a. A list of species encountered, the relationship between total length $\left(L_{\mathrm{T}}\right)$ and length at first maturity, length at first maturity in relation to maximum length $\left(L_{T \max }\right)$, and length at 50\% maturity (LT50) for Squaliformes: Centrophoridae, Etmopteridae, Somniosidae, and Dalatiidae.

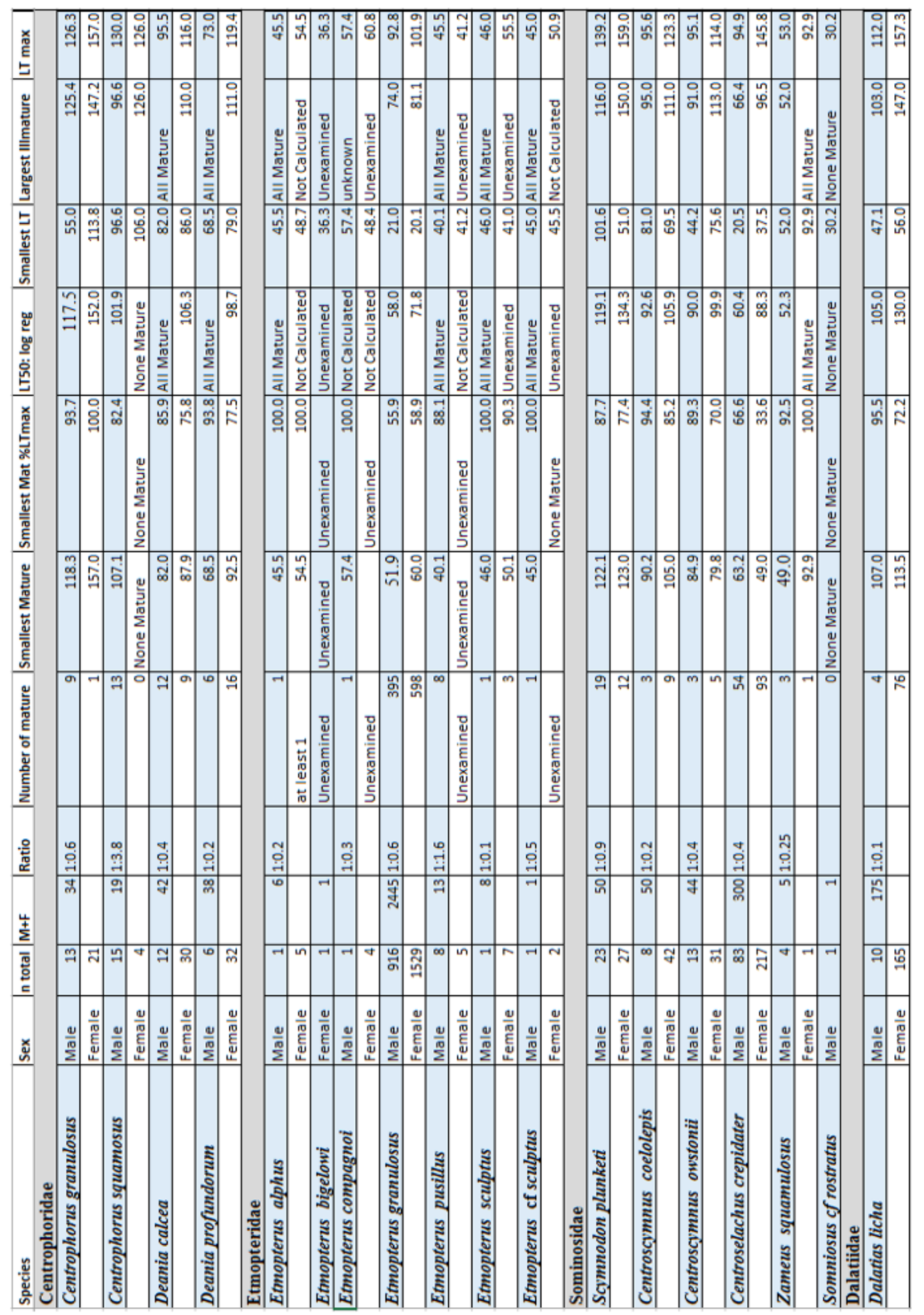


Table 10. Sex ratio significance evaluated by $\mathrm{p}$-value $<0.05$, and $\chi^{2}$ value for overall, adult, and subadult sex ratio.

\begin{tabular}{|c|c|c|c|c|c|c|}
\hline Species & $\begin{array}{l}\text { Maturit } \\
\mathbf{y}\end{array}$ & $\begin{array}{l}\text { Num. } \\
\text { female } \\
\text { s }\end{array}$ & $\begin{array}{l}\text { Num. } \\
\text { males }\end{array}$ & $\begin{array}{l}\text { P- } \\
\text { value }\end{array}$ & $\begin{array}{l}\text { Chi^^2 } \\
\text { value }\end{array}$ & $\begin{array}{l}\text { Significanc } \\
\text { e }\end{array}$ \\
\hline \multirow[t]{3}{*}{ Centrophorus granulosus } & Overall & 21 & 13 & 0.17 & 1.882 & $\begin{array}{l}\text { Not } \\
\text { significant }\end{array}$ \\
\hline & Adult & 1 & 9 & 0.011 & 6.4 & Significant \\
\hline & Subadult & 20 & 4 & 0.001 & 10.667 & Significant \\
\hline \multirow[t]{3}{*}{ Centrophorus squamosus } & Overall & 4 & 15 & 0.012 & 6.368 & Significant \\
\hline & Adult & 0 & 12 & $\begin{array}{l}<0.00 \\
1\end{array}$ & 13 & Significant \\
\hline & Subadult & 4 & 2 & 0.414 & 0.667 & $\begin{array}{l}\text { Not } \\
\text { significant }\end{array}$ \\
\hline \multirow[t]{3}{*}{ Deania calcea } & Overall & 30 & 12 & 0.005 & 7.714 & Significant \\
\hline & Adult & 9 & 12 & 0.513 & 0.513 & $\begin{array}{l}\text { Not } \\
\text { s ignificant }\end{array}$ \\
\hline & Subadult & 21 & 0 & $\begin{array}{l}<0.00 \\
1\end{array}$ & 21 & Significant \\
\hline \multirow[t]{3}{*}{ Deania profundorum } & Overall & 32 & 6 & $\begin{array}{l}<0.00 \\
1\end{array}$ & 17.789 & Significant \\
\hline & Adult & 16 & 6 & 0.033 & 4.545 & Significant \\
\hline & Subadult & 16 & 0 & $\begin{array}{l}<0.00 \\
1\end{array}$ & 16 & Significant \\
\hline \multirow[t]{3}{*}{ Etmopterus granulosus } & Overall & 1529 & 916 & $\begin{array}{l}<0.00 \\
1\end{array}$ & $\begin{array}{r}153.68 \\
9\end{array}$ & Significant \\
\hline & Adult & 598 & 395 & $\begin{array}{l}<0.00 \\
1\end{array}$ & 41.499 & Significant \\
\hline & Subadult & 931 & 521 & $\begin{array}{l}<0.00 \\
1\end{array}$ & $\begin{array}{r}115.77 \\
1\end{array}$ & Significant \\
\hline \multirow[t]{3}{*}{ Etmopterus lucifer } & Overall & 5 & 1 & 0.102 & 2.667 & $\begin{array}{l}\text { Not } \\
\text { significant }\end{array}$ \\
\hline & Adult & & & & & \\
\hline & Subadult & & & & & \\
\hline \multirow[t]{3}{*}{ Etmopterus molleri } & Overall & 2 & 1 & 0.564 & 0.333 & $\begin{array}{l}\text { Not } \\
\text { significant }\end{array}$ \\
\hline & Adult & & & & & \\
\hline & Subadult & & & & & \\
\hline \multirow[t]{3}{*}{ Etmopterus pusillus } & Overall & 5 & 8 & 0.405 & 0.692 & $\begin{array}{l}\text { Not } \\
\text { significant }\end{array}$ \\
\hline & Adult & & & & & \\
\hline & Subadult & & & & & \\
\hline \multirow[t]{2}{*}{ Etmopterus sculptus } & Overall & 7 & 1 & 0.034 & 4.5 & Significant \\
\hline & Adult & & & & & \\
\hline
\end{tabular}




\begin{tabular}{|c|c|c|c|c|c|c|}
\hline & Subadult & & & & & \\
\hline \multirow[t]{3}{*}{ Scymnodon plunketi } & Overall & 27 & 23 & 0.572 & 0.32 & $\begin{array}{l}\text { Not } \\
\text { significant }\end{array}$ \\
\hline & Adult & 12 & 19 & 0.209 & 1.581 & $\begin{array}{l}\text { Not } \\
\text { significant }\end{array}$ \\
\hline & Subadult & 15 & 4 & 0.012 & 6.368 & Significant \\
\hline \multirow[t]{3}{*}{ Centroscymnus coelolepis } & Overall & 42 & 8 & $\begin{array}{l}<0.00 \\
1\end{array}$ & 23.12 & Significant \\
\hline & Adult & 9 & 3 & 0.083 & 3 & $\begin{array}{l}\text { Not } \\
\text { significant }\end{array}$ \\
\hline & Subadult & 33 & 5 & $\begin{array}{l}<0.00 \\
1\end{array}$ & 20.632 & Significant \\
\hline \multirow[t]{3}{*}{ Centroscymnus owstonii } & Overall & 31 & 13 & 0.007 & 7.364 & Significant \\
\hline & Adult & 5 & 3 & 0.48 & 0.5 & Significant \\
\hline & Subadult & 26 & 10 & 0.008 & 7.111 & Significant \\
\hline \multirow[t]{3}{*}{ Centroselachus crepidater } & Overall & 217 & 83 & $\begin{array}{l}<0.00 \\
1\end{array}$ & 59.853 & Significant \\
\hline & Adult & 93 & 54 & 0.001 & 10.347 & Significant \\
\hline & Subadult & 124 & 29 & $\begin{array}{l}<0.00 \\
1 \\
\end{array}$ & 58.987 & Significant \\
\hline \multirow[t]{3}{*}{ Zameus squamulosus } & Overall & 1 & 4 & 0.18 & 1.8 & $\begin{array}{l}\text { Not } \\
\text { significant }\end{array}$ \\
\hline & Adult & & & & & \\
\hline & Subadult & & & & & \\
\hline \multirow[t]{3}{*}{ Dalatias licha } & Overall & 165 & 10 & $\begin{array}{l}<0.00 \\
1\end{array}$ & $\begin{array}{r}137.28 \\
6 \\
\end{array}$ & Significant \\
\hline & Adult & 76 & 4 & $\begin{array}{l}<0.00 \\
1\end{array}$ & 64.8 & Significant \\
\hline & Subadult & 89 & 6 & $\begin{array}{l}<0.00 \\
1\end{array}$ & 72.516 & Significant \\
\hline \multirow[t]{3}{*}{ Apristurus sinensis } & Overall & 34 & 59 & 0.01 & 6.72 & Significant \\
\hline & Adult & & & & & \\
\hline & Subadult & & & & & \\
\hline \multirow[t]{3}{*}{$\begin{array}{l}\text { Apristurus sp. cf. } \\
\text { albisoma }\end{array}$} & Overall & 5 & 3 & 0.48 & 0.5 & $\begin{array}{l}\text { Not } \\
\text { significant }\end{array}$ \\
\hline & Adult & & & & & \\
\hline & Subadult & & & & & \\
\hline \multirow[t]{3}{*}{$\begin{array}{l}\text { Apristurus sp. cf. } \\
\text { ampliceps } 1\end{array}$} & Overall & 5 & 2 & 0.257 & 1.286 & $\begin{array}{l}\text { Not } \\
\text { significant }\end{array}$ \\
\hline & Adult & & & & & \\
\hline & Subadult & & & & & \\
\hline \multirow[t]{3}{*}{$\begin{array}{l}\text { Apristurus sp. cf. } \\
\text { ampliceps } 2\end{array}$} & Overall & 3 & 1 & 0.317 & 1 & $\begin{array}{l}\text { Not } \\
\text { significant }\end{array}$ \\
\hline & Adult & & & & & \\
\hline & Subadult & & & & & \\
\hline Apristurus sp. cf. manis & Overall & 11 & 12 & 0.835 & 0.043 & $\begin{array}{l}\text { Not } \\
\text { significant }\end{array}$ \\
\hline
\end{tabular}




\begin{tabular}{|c|c|c|c|c|c|c|}
\hline & Adult & & & & & \\
\hline & Subadult & & & & & \\
\hline \multirow[t]{3}{*}{$\begin{array}{l}\text { Apristurus sp. cf. } \\
\text { melanoasper }\end{array}$} & Overall & 2 & 3 & 0.655 & 0.2 & $\begin{array}{l}\text { Not } \\
\text { significant }\end{array}$ \\
\hline & Adult & & & & & \\
\hline & Subadult & & & & & \\
\hline \multirow[t]{3}{*}{ Bythaelurus naylori } & Overall & 15 & 14 & 0.853 & 0.034 & $\begin{array}{l}\text { Not } \\
\text { significant }\end{array}$ \\
\hline & Adult & & & & & \\
\hline & Subadult & & & & & \\
\hline \multirow[t]{3}{*}{ Bythaelurus bachi } & Overall & 11 & 1 & 0.004 & 8.333 & Significant \\
\hline & Adult & & & & & \\
\hline & Subadult & & & & & \\
\hline \multirow[t]{3}{*}{ Pseudotriakis microdon } & Overall & 10 & 18 & 0.131 & 2.286 & $\begin{array}{l}\text { Not } \\
\text { significant }\end{array}$ \\
\hline & Adult & 2 & 9 & 0.035 & 4.455 & Significant \\
\hline & Subadult & 8 & 9 & 0.808 & 0.059 & $\begin{array}{l}\text { Not } \\
\text { significant }\end{array}$ \\
\hline \multirow[t]{3}{*}{ Chimaera willwatchi } & Overall & 35 & 17 & 0.017 & 5.667 & Significant \\
\hline & Adult & 7 & 5 & 0.564 & 0.333 & $\begin{array}{l}\text { Not } \\
\text { significant }\end{array}$ \\
\hline & Subadult & 30 & 12 & 0.008 & 7.049 & Significant \\
\hline \multirow[t]{3}{*}{ Hydrolagus sp A } & Overall & 2 & 1 & 0.564 & 0.333 & $\begin{array}{l}\text { Not } \\
\text { significant }\end{array}$ \\
\hline & Adult & 1 & 1 & & & $\begin{array}{l}\text { Not } \\
\text { significant }\end{array}$ \\
\hline & Subadult & & 1 & & & $\begin{array}{l}\text { Not } \\
\text { significant }\end{array}$ \\
\hline
\end{tabular}

Females ranged from 113.8 to $157 \mathrm{~cm} L_{\mathrm{T}}$, with a single mature individual that was also the largest female encountered (Figure 23-c). Oviducal gland width increased between 147 and $157 \mathrm{~cm} L_{\mathrm{T}}$ (Figure 23-d) and $L_{\mathrm{T} 50}$ was estimated to be $152.0 \mathrm{~cm}$. The mature female had a total number of 29 mature oocytes evenly distributed between left (14 oocytes) and right (15 oocytes) ovaries, with a maximum diameter of $0.8 \mathrm{~cm}$. 

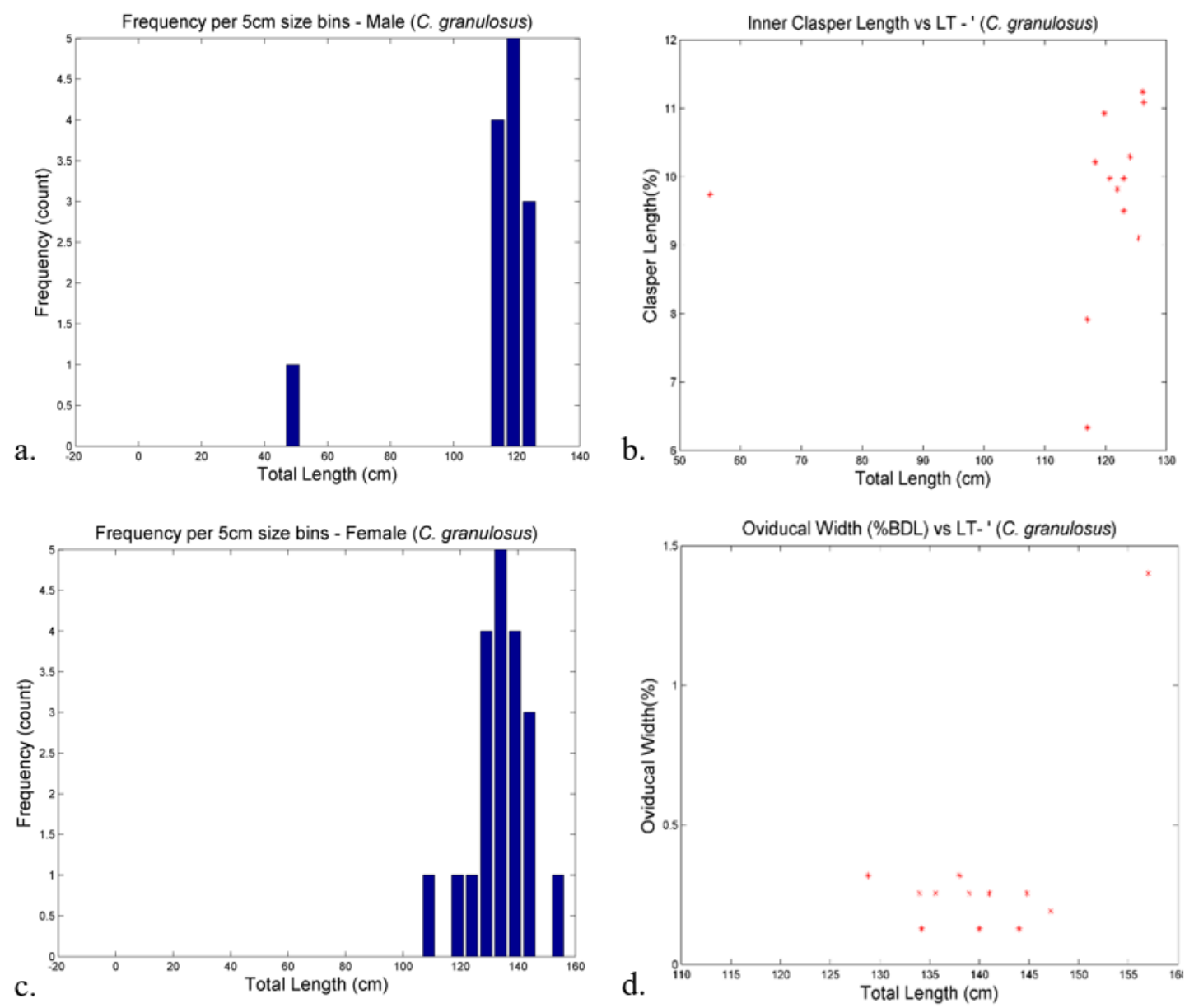

Figure 23. Centrophorus granulosus: a) size distribution of males, b) relationship between inner clasper length (\%LT) and LT (males), c) size distribution of females, d) relationship between shell gland width (\%LT) and LT (females) Diet for this species was exclusively composed of bony fishes, which were found in six of the stomachs examined.

Males and females were taken from seamounts along both Walters Shoal and the Southwest Indian Ocean Ridge at depths between 820 and 1312 m (Figure 24). 


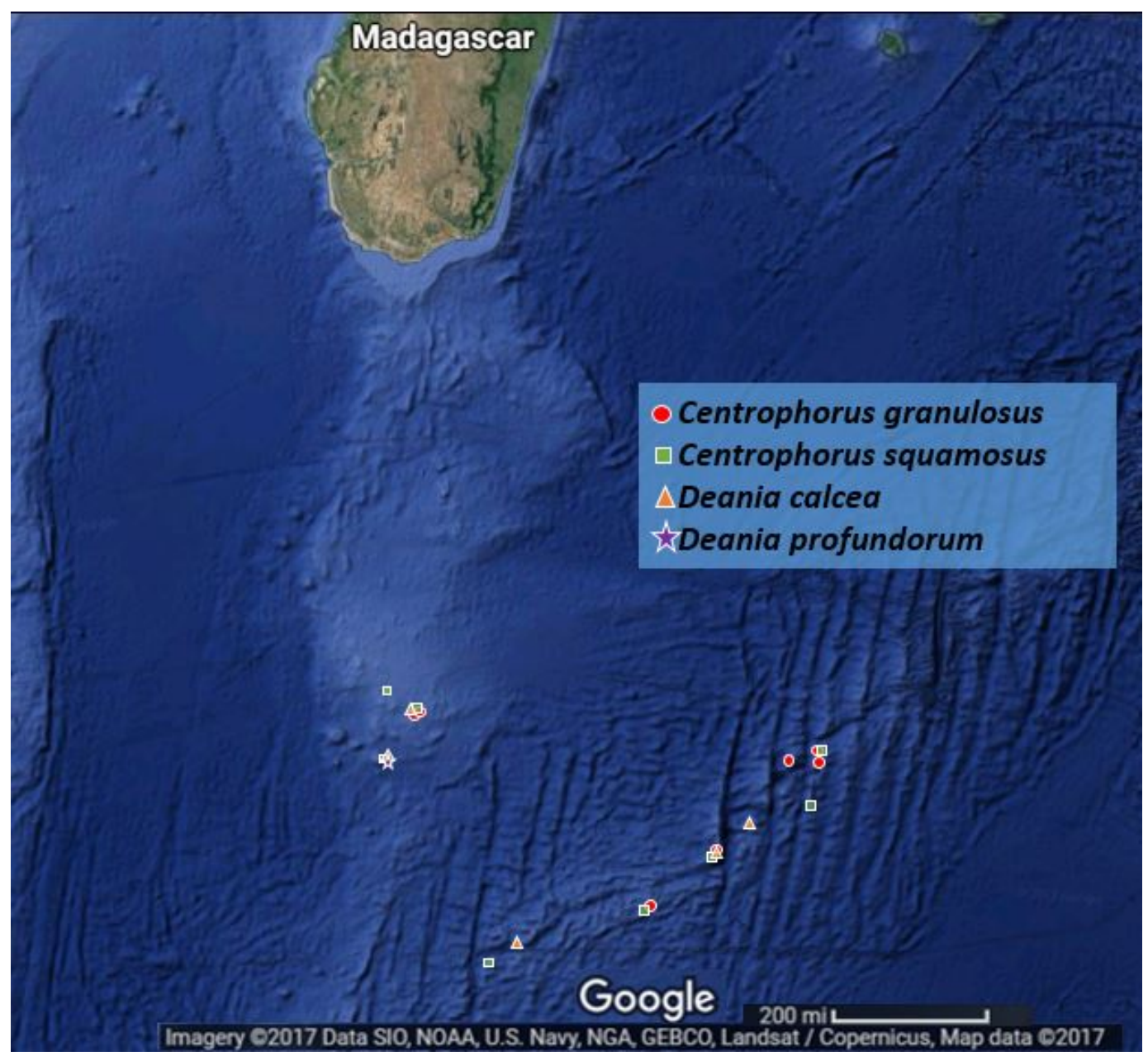

Figure 24. Distribution of the family Centrophoridae of this study: Centrophorus granulosus (red circle), Centrophorus squamosus (green square), Deania calcea (orange triangle), and Deania profundorum (purple star). Map data: Google, Image (c) 2017 DigitalGlobe.

Centrophorus squamosus (Bonnaterre, 1788), Leftscale Gulper Shark. A total of 19 C. squamosus (four females and 15 males) were examined with a F:M ratio of 1:3.8, significantly different from the expected equal ratio (p-value<0.05). Comparison by maturity shows adults were exclusively male (p-value<0.05), and while juveniles were dominated by females 1:0.5 the sample size was too small to show significance (pvalue $>0.05)$. 
Males ranged from $96.6 \mathrm{~cm} L_{\mathrm{T}}$ to $130 \mathrm{~cm} L_{\mathrm{T}}$, with 13 mature $(86.7 \%$ of those encountered) (Figure 25-a). The smallest mature measured $107.1 \mathrm{~cm} L_{\mathrm{T}}$ and largest immature male measured $96.6 \mathrm{~cm} L_{\mathrm{T}}$. Claspers length increases between $96 \mathrm{~cm} L_{\mathrm{T}}$ and $105 \mathrm{~cm} L_{\mathrm{T}}$ (Figure 25-b), the smallest mature was at $82.4 \% L_{\mathrm{T}} \max$, and $L_{\mathrm{T} 50}$ was estimated to be $101.9 \mathrm{~cm} L_{\mathrm{T}}$. Females ranged from $106 \mathrm{~cm} L_{\mathrm{T}}$ to $126 \mathrm{~cm} L_{\mathrm{T}}$, but no mature individuals were encountered.

a.
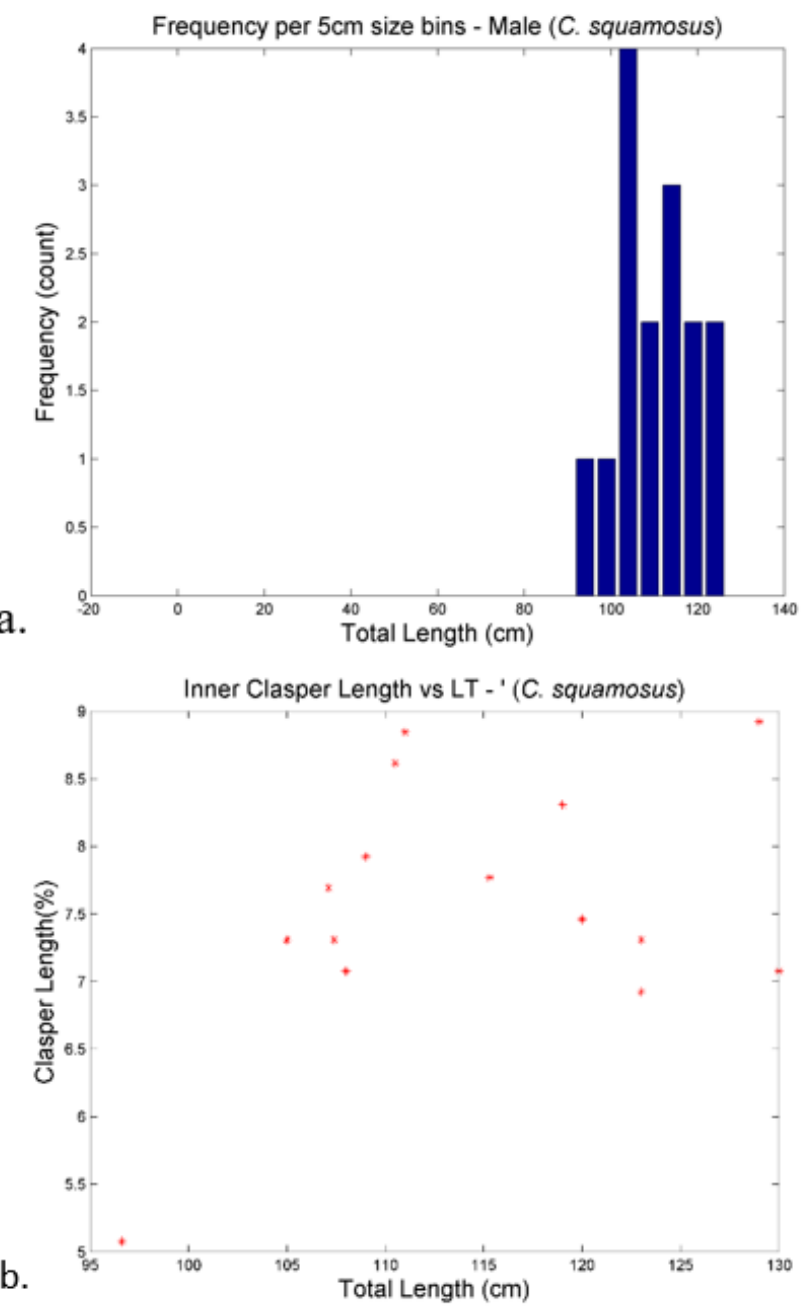

Figure 25. Centrophorus squamosus: a) size distribution of males, b) relationship between inner clasper length (\%Lт) and $\mathrm{L}_{\mathrm{T}}$ (males).

One male had cephalopod remains in its stomach. 
Males and females were encountered off Walters Shoal and the Southwest Indian Ocean Ridge at depths between 495 and 1250 m (Figure 24).

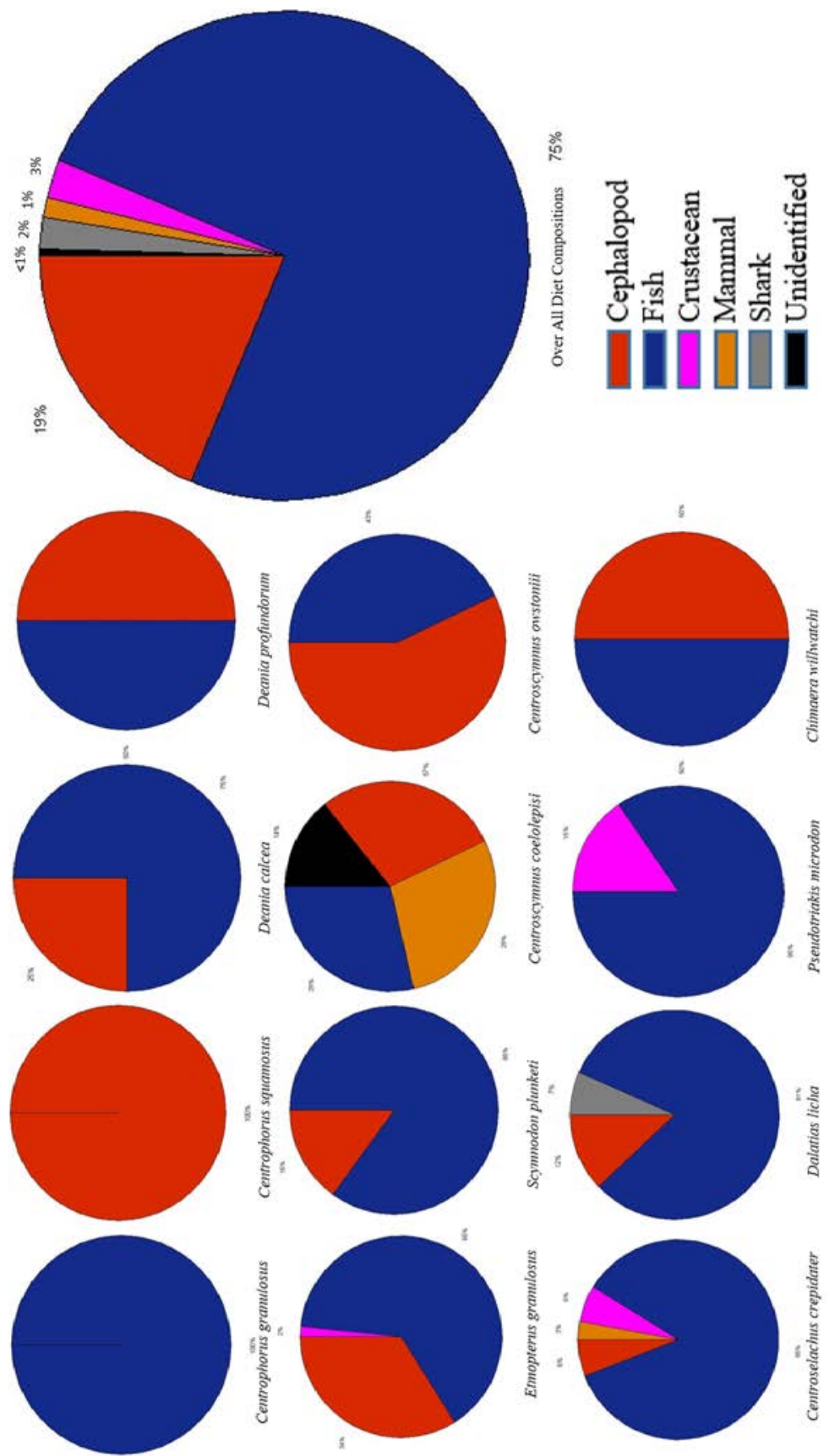

Figure 26. Pie chart of prey items presented as percent composition of item-count for each species encountered with stomach contents. Broad taxonomic groups are shown as: cephalopod (red), fish (blue), crustacean (pink), mammal (orange), shark (grey), and unidentified (black). 
Deania calcea (Lowe, 1839), Birdbeak Dogfish. In all, 42 D. calcea (30 females and 12 males) were collected with an overall female to male ratio of 1:0.4, which is significantly different from the null hypothesis of a 1:1 ratio (p-value $<0.05)$. Comparison by maturity level revealed an insignificant majority of adults were male, 1:1.3 (pvalue $>0.05$ ), while the 21 juveniles were exclusively female.

Males ranged from 82.0 to $95.5 \mathrm{~cm} L_{\mathrm{T}}$ and were all determined to be mature, with the smallest individual measuring $82.0 \mathrm{~cm}$ or $5.9 \%$ of the $L_{\mathrm{Tmax}}$ (Figure $27-\mathrm{a}$ ). Females ranged from 86 to $116 \mathrm{~cm} L_{\mathrm{T}}$, with nine mature, the smallest of which measured $87.9 \mathrm{~cm}$ $L_{\mathrm{T}}\left(75.8 \% L_{\mathrm{Tmax}}\right)$, and the largest immature measured $110 \mathrm{~cm} L_{\mathrm{T}}$ (Figure 27-b). Oviducal gland width increased between $95 \mathrm{~cm} L_{\mathrm{T}}$ and $98 \mathrm{~cm} L_{\mathrm{T}}$ (Figure 27-c), with $L_{\mathrm{T} 50}$ estimated to be $106.3 \mathrm{~cm} L_{\mathrm{T}}$. 

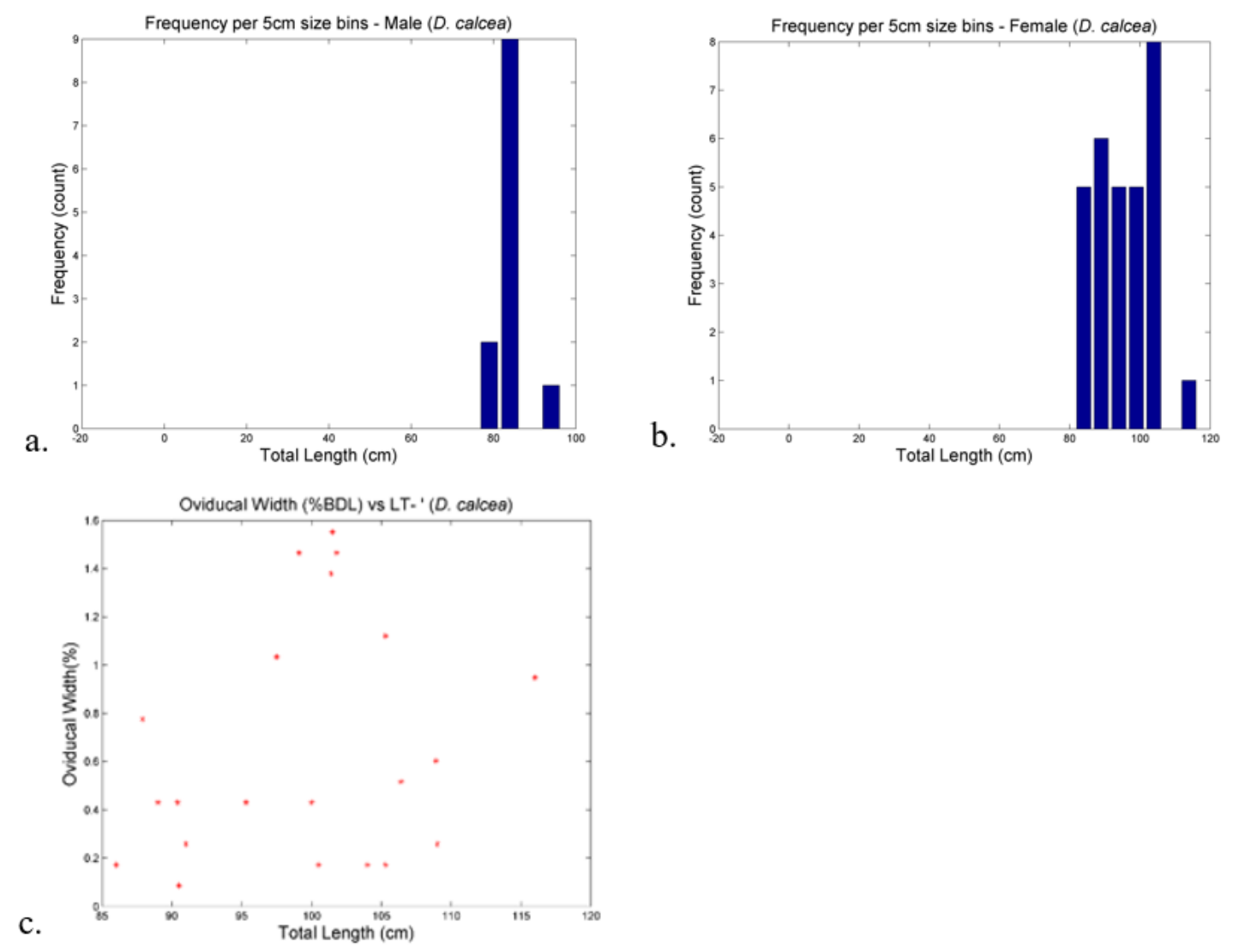

Figure 27. Deania calcea: a) size distribution of males, b) size distribution of females, c) relationship between shell gland width (\%LT) and LT (females).

Females had between four and 18 eggs (averaging 8.6) in the left uterus and three to 12 eggs (averaging 7.8) in the right uterus; this discrepancy was not significantly different from unity (p-value >0.05) (Table 11). The largest mature egg had a maximum diameter of $4.7 \mathrm{~cm}$.

Two females had bony fishes in their stomachs, with a third female containing a mix of fish and cephalopods.

Although juvenile males and females were sometimes encountered on the same seamounts between 503 and 1290 meters, mature males were encountered along the 
Southwest Indian Ocean Ridge (with one exception) while all mature females (except the largest female encountered, $108.9 \mathrm{~cm}$ ) were encountered along Walters Shoal (Figure 24).

Table 11. List of numbers of ovarian oocytes (average and max), uterine eggs (left max, right max, total max, and average), and max width of oocytes

\begin{tabular}{|c|c|c|c|c|c|c|c|}
\hline Species & Ovarian oocytes average count & Ovarian oocytes max count & Cteriue eggs max width & Uterime egggs mas count lef & Uteriue eggs max count right & Uterize eggs average count & Mas egges \\
\hline Deania calcea & 9.6 & 20 & \begin{tabular}{|l|}
4.7 \\
\end{tabular} & 18 & 12 & 7.3 & 30 \\
\hline Deamia profundorum & 9.2 & 11 & 6.2 & 17 & 15 & 7.3 & 32 \\
\hline Etmopterus gramulosus & 8.7 & 38 & 5.3 & 24 & 38 & 6.8 & 54 \\
\hline Scymuodon plunketi & 17.1 & 16.6 & 7.5 & 36 & 30 & 11.5 & 43 \\
\hline Centroscymuns coelolepis & 249 & 45 & 59 & 22 & 20 & 11 & 42 \\
\hline Centroscymunus anstonii & 11 & 18 & 62 & 15 & 14 & 104 & 22 \\
\hline Centroselachus crepidater & 5.9 & 24 & 3.5 & 16 & 14 & 5.2 & 30 \\
\hline Dalatias licha & 6.7 & 24 & 9.2 & 26 & 15 & 6.5 & 35 \\
\hline
\end{tabular}

Deania profundorum (Smith \& Radcliffe, 1912), Arrowhead Dogfish. Collectively 38 D. profundorum (32 females and six males) were encountered with an overall F:M sex ratio of 1:0.2, significantly favoring females ( $\mathrm{p}$-value<0.05). Comparison by maturity level showed most adults, 1:0.4 (p-value<0.05), and all juveniles were female.

Males ranged from 68.5 to $73 \mathrm{~cm} L_{\mathrm{T}}$, and were all determined to be mature. Females ranged from 79 to $119.4 \mathrm{~cm} L_{\mathrm{T}}$, with 16 individuals (50\%) mature (Figure 28-a). Oviducal gland width increased between $92 \mathrm{~cm} L_{\mathrm{T}}$ and $93 \mathrm{~cm} L_{\mathrm{T}}$ (Figure 28-b). Smallest mature measured $92.5 \mathrm{~cm} L_{\mathrm{T}}\left(77.4 \% L_{\mathrm{T} \max }\right)$, the largest immature female measured 111 cm $L_{\mathrm{T}}$, and $L_{\mathrm{T} 50}$ was estimated to occur at $98.7 \mathrm{~cm} L_{\mathrm{T}}$. 

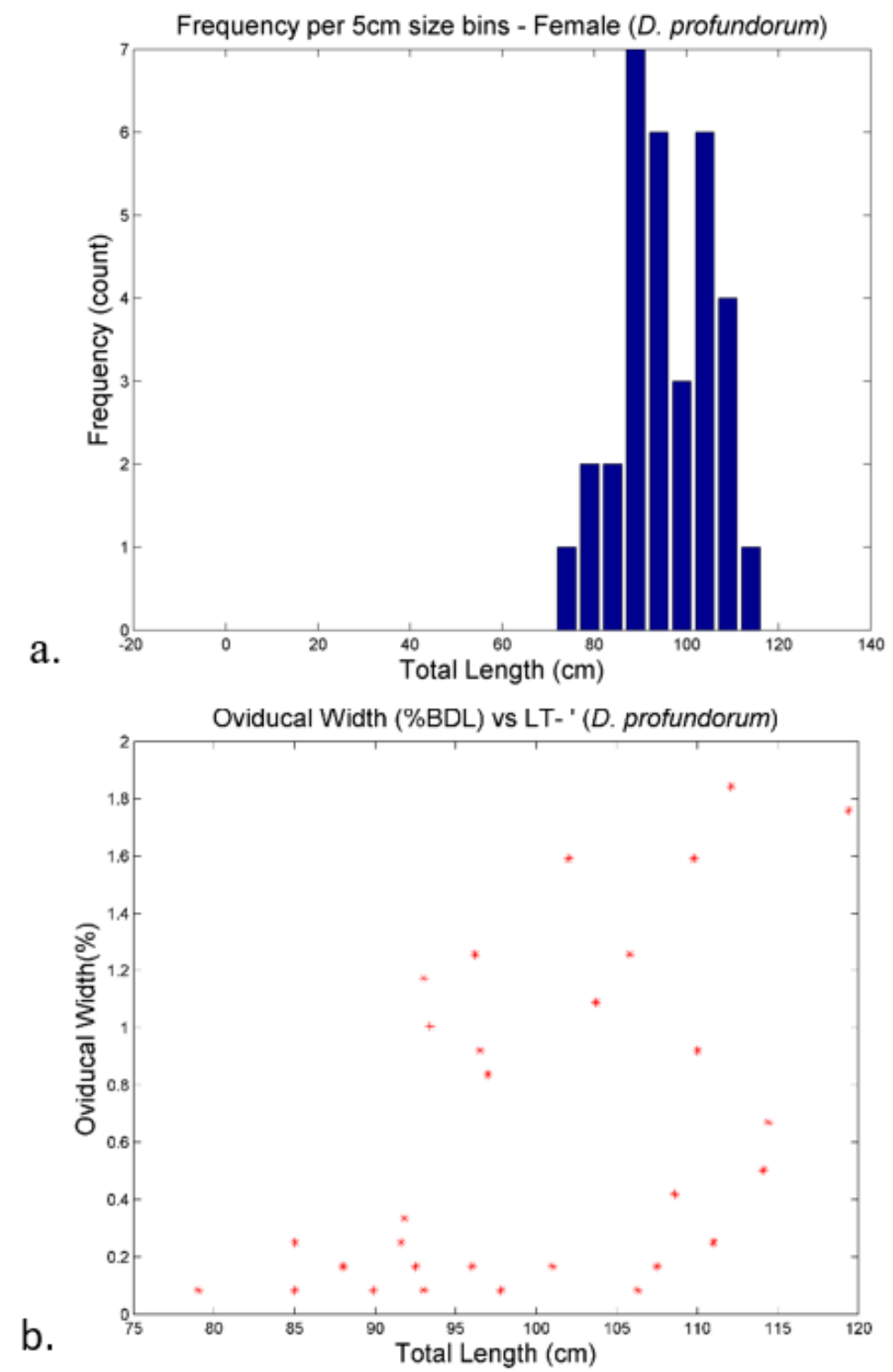

Figure 28. Deania profundorum: a) size distribution of females, b) relationship between shell gland width (\%LT) and LT (females).

Females had a total average of 15 mature oocytes, with between two and 17 oocytes in their left ovary (averaging 7.3) and three to 15 in their right (averaging 7.6) with insignificant differences between left and right ovaries (p-value $>0.05$ ). The largest mature oocytes had a maximum diameter of $6.2 \mathrm{~cm}$. 
Stomach contents were found in two females and consisted of bony fish in an adolescent and squid in the adult.

Males and females were found on a single seamount in the Walters Shoal seamount complex, with a depth range of 560 to $1290 \mathrm{~m}$ (Figure 24).

Etmopterus alphus, Ebert, Straube, Leslie, and Weigman, 2016, White Cheek Lanternshark. A total of six E. alphus were collected (five females and one male), with an overall sex ratio of 1:0.2, favoring females but a sample size too small to be significant (p-value $>0.05$ ). The only male was mature at $45.5 \mathrm{~cm} L_{T}$. Females ranged from 48.7 to $54.5 \mathrm{~cm} L_{\mathrm{T}}$, the largest of which was pregnant. Internal maturity indicators and diet were not examined.

Females were collected from both Walters Shoal and the Southwest Indian Ocean Ridge (Figure 29) with a depth range of 500 and $1300 \mathrm{~m}$, and the only male was collected from Walters Shoal between 900 and 1200 m deep. 


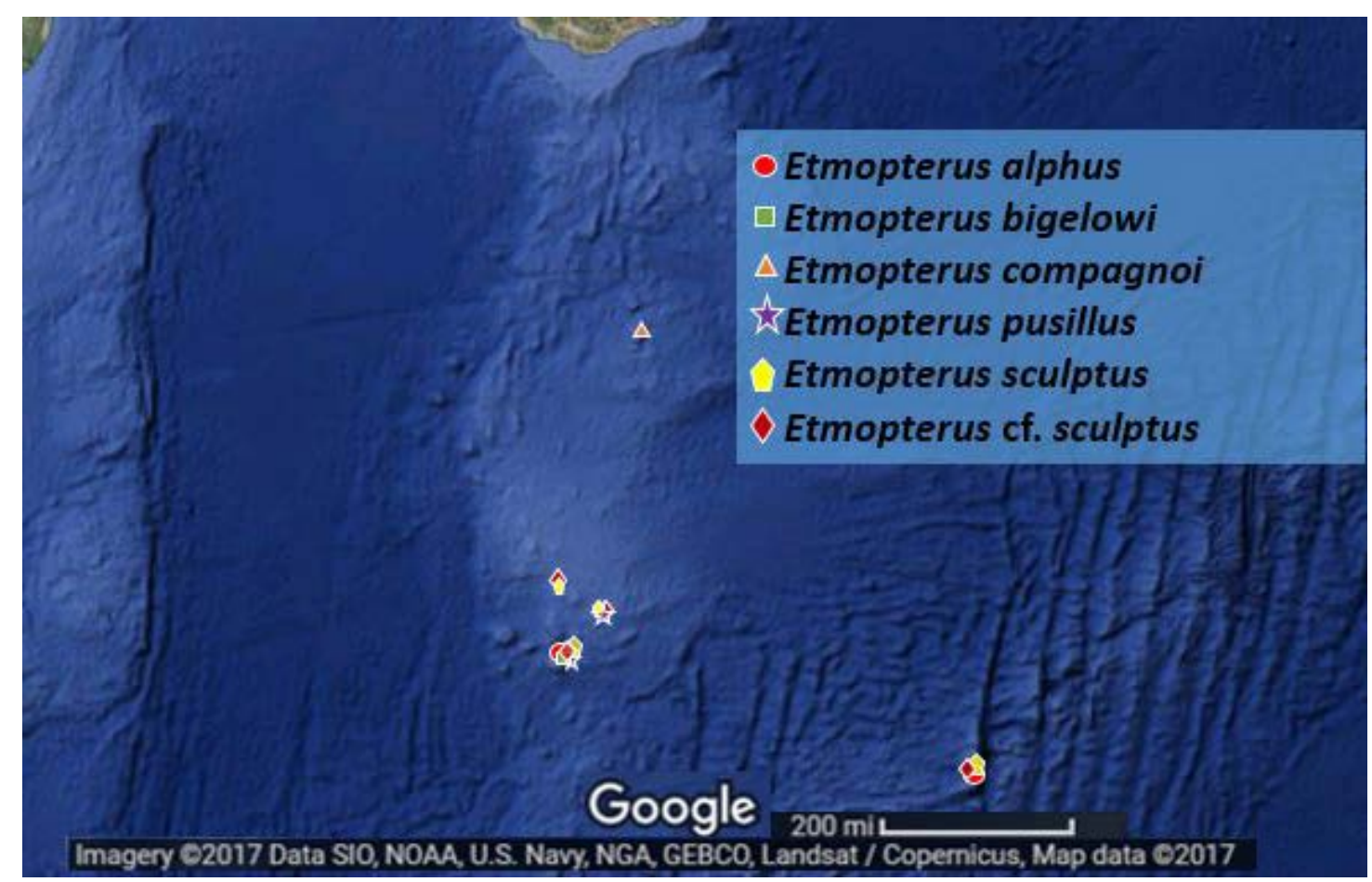

Figure 29. Distribution of the family Etmopteridae (sans E. granulosus. See figure 30): Etmopterus alphus (red circle), E. bigelowi (green square), E. compagnoi (orange triangle), E. pusillus (purple star), E. sculptus (yellow pentagon), E. cf. sculptus (maroon diamond). Map data: Google, Image (C) 2017 DigitalGlobe.

Etmopterus bigelowi, Shirai \& Tachikawa, 1993, Blurred Smooth Lantern Shark. A single female specimen measuring $36.3 \mathrm{~cm} L_{\mathrm{T}}$ was encountered from Walters Shoal between 560 and 1007 m deep. To maintain the integrity of the specimen it was not internally assessed for maturity (Figure 29).

Etmopterus compagnoi, Fricke and Koch, 1990, Brown Lanternshark. In all, five E. compagnoi (four females and one male) were collected, with an overall F:M of 1:0.3 (pvalue >0.05). The male was mature at $57.4 \mathrm{~cm} L_{\mathrm{T}}$, while the four females ranged from 48.4 to $60.8 \mathrm{~cm} L_{\mathrm{T}}$, and were not examined internally. This species was only encountered in the northern region of the Madagascar Ridge between 800 and 1300 m deep (Figure 29). 
Etmopterus granulosus (Günther, 1880), Southern Lanternshark. A total of 2445 (1529 females and 916 males) were examined, with an overall F:M ratio of 1:0.6, with significantly more females collected ( $\mathrm{p}$-value $<0.05$ ). Comparison by maturity status shows significantly more adult females, 1:0.6 ( $\mathrm{p}$-value $<0.05$ ), and more juvenile females 1:0.5 (p-value $<0.05)$.

Males ranged from 21 to $92.8 \mathrm{~cm} L_{\mathrm{T}}$, with 395 mature individuals ( $43.1 \%$ of examined) (Figure 30-a). Clasper length increased between 51 and $52 \mathrm{~cm} L_{\mathrm{T}}$ (Figure 30b). The smallest mature male measured $51.9 \mathrm{~cm} L_{\mathrm{T}}$ or $55.9 \% L_{\mathrm{T} \max }$, the largest immature was $74 \mathrm{~cm} L_{\mathrm{T}}$, and $L_{\mathrm{T} 50}$ was estimate at $58.0 \mathrm{~cm} L_{\mathrm{T}}$. Females ranged from 20.1 to 101.9 cm $L_{\mathrm{T}}$, with 598 mature (39.1\% examined) (Figure 30-c). Oviducal gland width increased sharply between $63 \mathrm{~cm} L_{\mathrm{T}}$ and $68 \mathrm{~cm} L_{\mathrm{T}}$ (Figure 30d). The smallest mature female measured $60 \mathrm{~cm} L_{\mathrm{T}}\left(58.9 \% L_{\mathrm{T} \max }\right)$, the largest immature at $81.1 \mathrm{~cm} L_{\mathrm{T}}$, and $L_{\mathrm{T} 50}$ was estimated at $71.8 \mathrm{~cm} \mathrm{LT}$. 

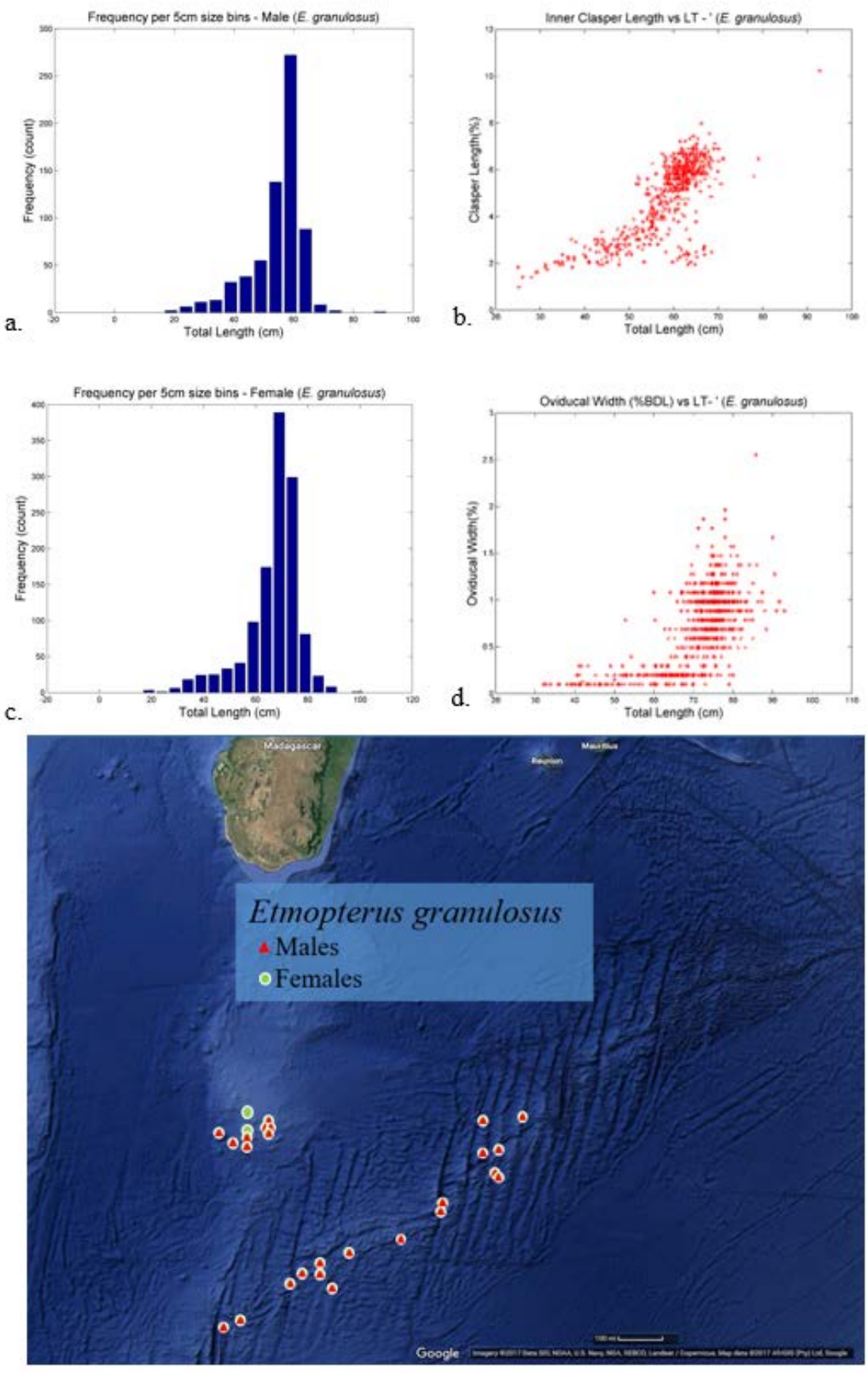

e.

Figure 30. Etmopterus granulosus: a) size distribution of males, b) relationship between inner clasper length (\% $\left.\mathrm{L}_{\mathrm{T}}\right)$ and $\mathrm{L}_{\mathrm{T}}$ (males), c) size distribution of the females, d) relationship between shell gland width (\%LT) and $\mathrm{LT}_{\mathrm{T}}$ (females), and e) distribution of males (red triangles) and females (green circles). Map data: Google, Image (c) 2017 DigitalGlobe. 
Females had 14 mature oocytes on average with between one and 38 oocytes per ovary, averaging 7.1 left and 6.5 right (p-value <0.05). Uterine eggs ranged from 1 to 38, averaging 6.8 per uterus. There was no correlation between mother size and number of oocytes or eggs. The largest mature egg had a maximum diameter of $5.3 \mathrm{~cm}$. Forty-four pregnant females ranging from 64 to $90 \mathrm{~cm} L_{\mathrm{T}}$ with an average of $76.4 \mathrm{~cm} L_{\mathrm{T}}$ were encountered from a relatively large area throughout the Southwest Indian Ocean Ridge.

Pregnant females bore litters which were at different stages of development with a trend for larger pups from females measuring between 72 and $80 \mathrm{~cm} L_{\mathrm{T}}$. Number of pups ranged from 2 to 15, with an average of 7.9 pups per mother. Female pups outnumbered male pups by more than 1:0.65 (p-value<0.05), with male pups ranging from 5.4 to $22 \mathrm{~cm}$ $L_{\mathrm{T}}$ and females from 4.4 to $21.8 \mathrm{~cm} L_{\mathrm{T}}$. This ratio favored female pups consistently throughout length of mothers. Umbilical scars present on the smallest free-swimming male (21 cm $\left.L_{\mathrm{T}}\right)$ and female (20.1 $\left.\mathrm{cm} L_{\mathrm{T}}\right)$ suggest minimum length for the species.

Table 12. List of numbers of species bearing pups, number of pups, and number of pups reported in the literature

\begin{tabular}{|l|c|c|l|}
\hline Species & Number of Pups & Previously Reported & Reference \\
\hline Etmopterus granulosus & $2-15$ & $6-16$ & Wetherbee, 1996; Ebert, 2013 \\
\hline Scymnodon plunketi & 20 & 30 & Garrick, 1959a; Compagno, 1984; Ebert, 2013 \\
\hline Centroscymnus coelolepis & 12 & $1-29$ & Garrick, 1959b; Cox and Francis, 1997; Ebert, 2013 \\
\hline Centroselachus crepidater & $2-7$ & $1-9$ & Last and Stevens, 1994; Ebert, 2013 \\
\hline Zameus squamulosus & 3 & unreported & \\
\hline Pseudotriakis microdon & 2 & 2 & Yano, 1992; White et al, 2006; Ebert, 2013 \\
\hline
\end{tabular}

Of the 61 individuals with stomach contents, 39 (66\%) contained bony fishes and 20 (33\%) had cephalopods. Only two individuals were found to have both fish and cephalopod within their stomachs, and fish made up the majority (70\%) of the visually estimated volumes. Males fed mostly on cephalopods (80\%) with only a single male (63 
cm TL) having fish in its stomach. Females largely fed on fish (88.9\%). Diets often consisted of large pieces or whole animals.

This was by far the most commonly encountered and widespread elasmobranch of the survey. Males and females were caught along both the Walters Shoal and the Southwest Indian Ocean Ridge with a depth range of 89 to 1334 m, with no clear segregation (Figure 30-e). Pregnant females were present exclusively at Southwest Indian Ocean Ridge.

Etmopterus pusillus (Lowe, 1839), Smooth Lanternshark. A total of 13 E. pusillus (five females and eight males) were collected, with an overall F:M sex ratio of 1:1.6, not significantly different from unity ( $p$-value $>0.05$ ). Males ranged from 40.1 to $45.5 \mathrm{~cm} L_{\mathrm{T}}$, and were all determined to be mature. The smallest male was $88.1 \% L_{\mathrm{T} m a x}$. Females ranged from 41.2 to $51.6 \mathrm{~cm} L_{\mathrm{T}}$. Internal maturity indicators and diet was not examined.

Males and females were found together and only encountered along seamounts of Walters Shoal with a depth range of 580 to 1020 m (Figure 29). 
Table 13. List of species found with stomach contents, prey items given by number and percentage composition of estimated value, compared to diet reported in the literature.

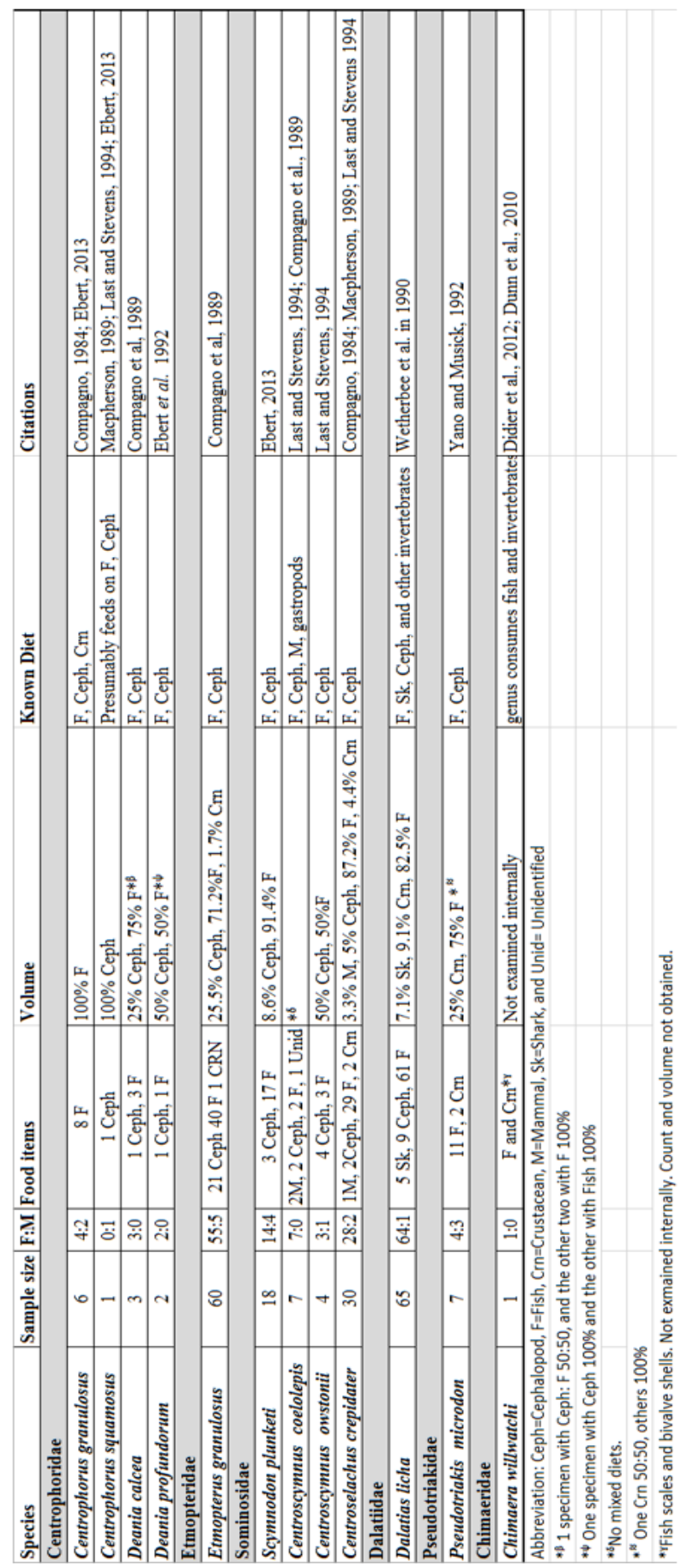


Etmopterus sculptus, Ebert, Compagno \& De Vries, 2011, Sculpted Lanternshark.

Eight E. sculptus (seven females and one male) were collected with an overall

F:M ratio of 1:0.1 significantly different to the expected null hypothesis ( $\mathrm{p}$-value $<0.05$ ). The only male was mature at $46 \mathrm{~cm} L_{\mathrm{T}}$. Females ranged from 41 to $55.5 \mathrm{~cm} L_{\mathrm{T}}$, with at least three pregnant individuals, the smallest of which measured $50.1 \mathrm{~cm} L_{\mathrm{T}}(90.3 \%$ $\left.L_{T \max }\right)$. Internal maturity indicators and diet were not examined.

This species was collected from two seamounts along the Walters Shoal between 495 and $1288 \mathrm{~m}$ deep (Figure 29).

Etmopterus cf. sculptus, Sculpted Lanternshark. Three specimens of E. cf. sculptus were collected (two females and one male), a F:M ratio of 1:0.5, not significantly different from unity (p-value $>0.05$ ). The single male was mature at $45 \mathrm{~cm} L_{\mathrm{T}}$. Females measured 45.5 and $50.9 \mathrm{~cm} L_{\mathrm{T}}$, and maturity was not evaluated internally.

Females were taken from the Southwest Indian Ocean Ridge, and the single male was encountered at Walters Shoal between 896 and 1300 m deep (Figure 29).

Scymnodon plunketi (Waite, 1910), Plunket's Shark. A total of 50 S. plunketi (27 females and 23 males) were encountered, with an overall F:M sex ratio of 1:0.9, not significantly different from the expected 1:1 (p-value $>0.05$ ). Comparison based on maturity level revealed adult males did not significantly outnumber adult females, F:M of 1:1.6 (p-value>0.05) but juvenile females were almost 3 times as abundant juvenile males, 1:0.3 (p-value <0.05).

Males ranged from 101.6 to $139.2 \mathrm{~cm} L_{\mathrm{T}}$, with 19 mature (82.6\% males encountered) (Figure 31-a). Clasper length increased sharply between 116 and $123 \mathrm{~cm} \mathrm{LT}$ (Figure 31- 
b), and the smallest mature male measured $122.1 \mathrm{~cm} L_{\mathrm{T}}\left(87.7 \% L_{\mathrm{T} \max }\right.$ ), the largest immature male measured $116 \mathrm{~cm} L_{\mathrm{T}}$, and $L_{\mathrm{T} 50}$ was estimated to be $119.1 \mathrm{~cm} L_{\mathrm{T}}$. Females ranged from $51 \mathrm{~cm}$ to $159 \mathrm{~cm} L_{\mathrm{T}}$, with 12 mature ( $44.4 \%$ encountered), the smallest maturing at $123 \mathrm{~cm} L_{\mathrm{T}}\left(77.4 \% L_{\mathrm{T} \max }\right.$ ) (Figure 31-c). Oviducal gland width increase between 124 and $139 \mathrm{~cm} L_{\mathrm{T}}$ (Figure 31-d), the largest immature female measured $150 \mathrm{~cm}$ $L_{\mathrm{T}}$, and $L_{\mathrm{T} 50}$ was estimated at $134.3 \mathrm{~cm} L_{\mathrm{T}}$.

a.
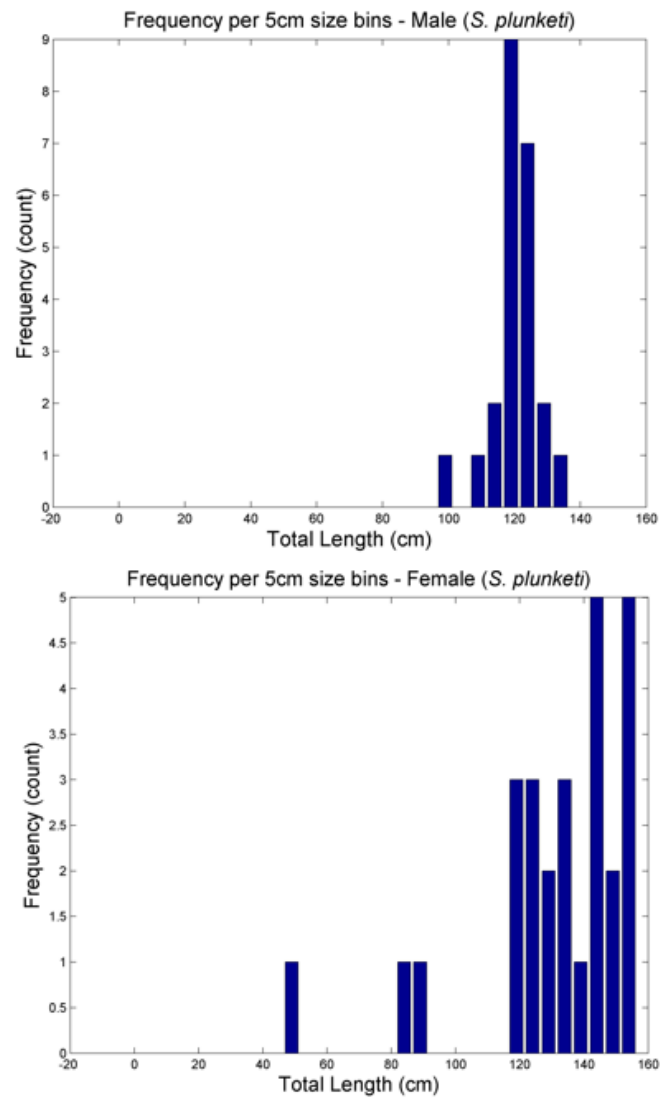

Oviducal Width (\%BDL) vs LT- ' (S. plunketi)

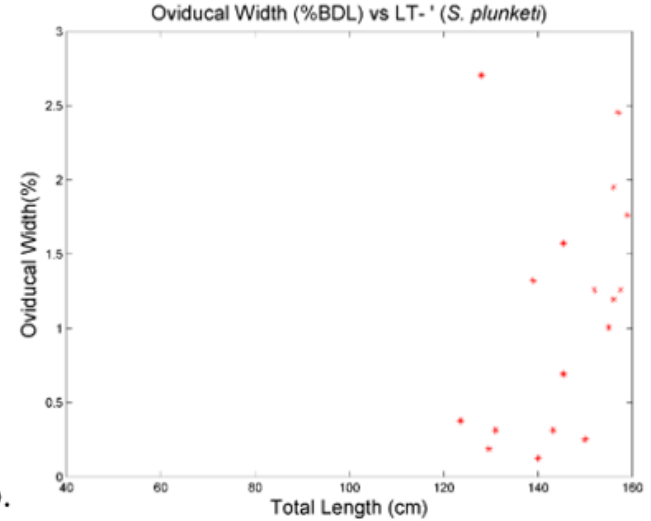

d.

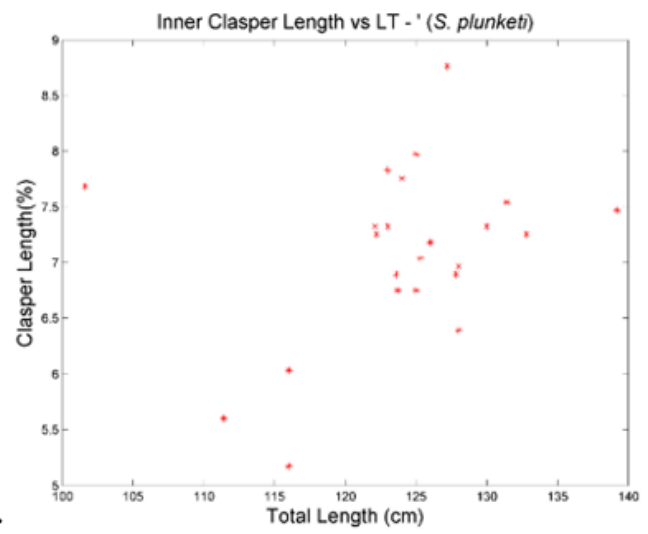

Figure 31. Scymnodon plunketi: a) size distribution of males, b) relationship between inner clasper length (\%LT) and LT (males), c) size distribution of females, d) relationship between shell gland width (\%LT) and LT (females).

Females had between 10 and 34 eggs evenly distributed between left and right uteri with between 10 and 36 oocytes in either ovary. The largest mature egg had a maximum diameter of $7.5 \mathrm{~cm}$. The single pregnant individual bore 20 pups (14 females and 6 
males), female pups outnumbering males 1:0.4, although statistically not significant (pvalue $>0.05$ ). Male pups ranged from 20.7 to $22.5 \mathrm{~cm} L_{\mathrm{T}}$, and female pups from 21 to 22.2 $\mathrm{cm} L_{\mathrm{T}}$.

Diet was comprised largely of bony fishes, with 14 (82.4\%) individuals feeding solely on fish, one (5.9\%) individual feeding on cephalopods, and two (11.8\%) with fish (66\% and $80 \%$ by volume) and cephalopods (34\% and $20 \%$ by volume) in its stomach.

Males were only encountered on the seamounts of the Southwest Indian Ocean Ridge with a range of 594 to 1288 m deep. Females were taken from both the Southwest Indian Ocean Ridge and Walters Shoal between 736 and 1271 m deep, with two mature individuals (7\% of females) from the same area as the males (Figure 32). 


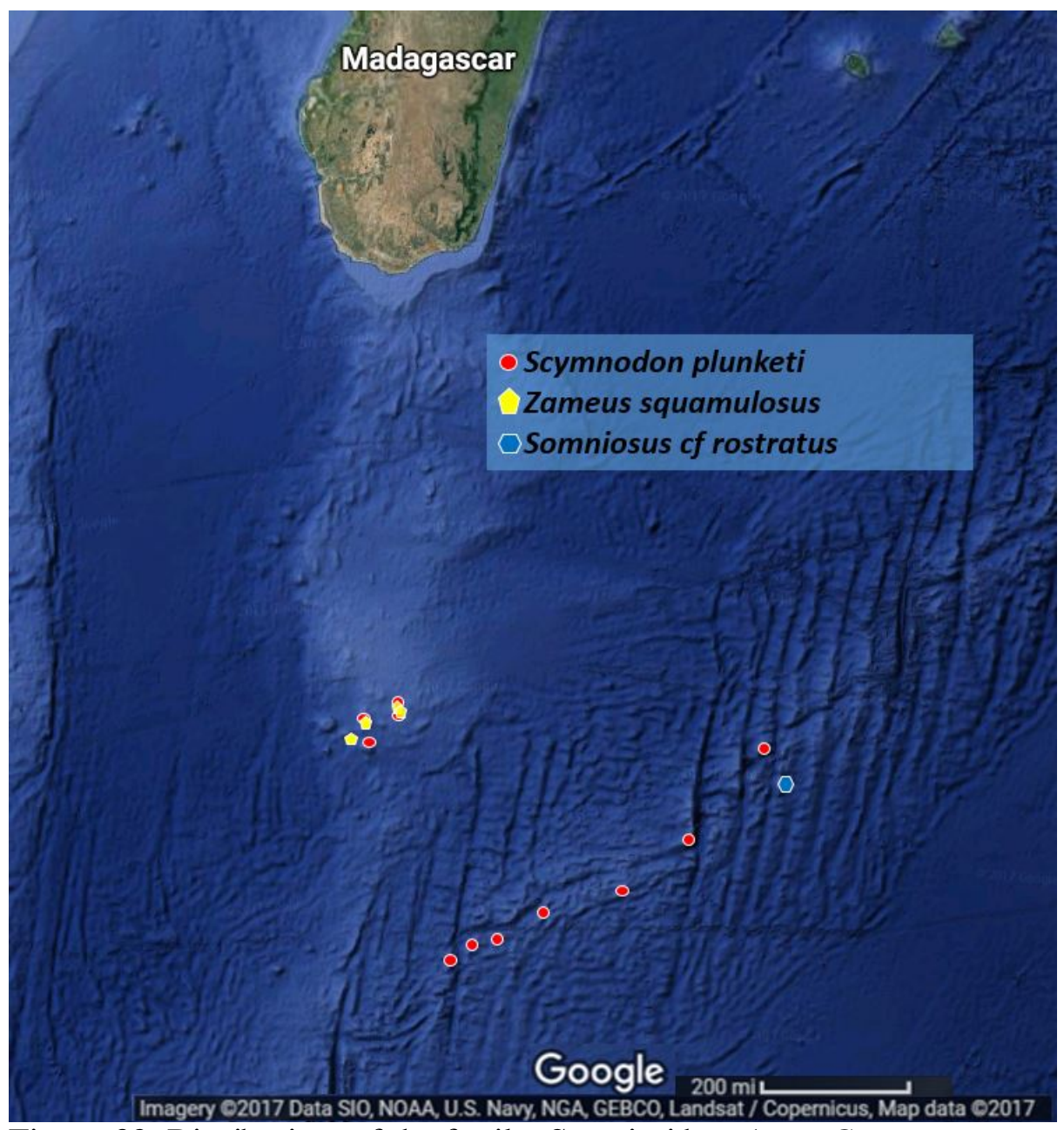

Figure 32. Distribution of the family Somniosidae (sans Centroscymnus, Centroselachus, and Dalatias): Scymnodon plunketi (red circle), Zameus squamulosus (yellow pentagon), and Somniosus cf rostratus (blue hexagon). Map data: Google, Image (c) 2017 DigitalGlobe.

Centroscymnus coelolepis Barbosa du Bocage \& de Brito Capello, 1864, Portuguese Dogfish. In all, 50 C. coelolepis (42 females and eight males) were collected with a F:M ratio of 1:0.2, with significantly more females than the expected 1:1 even sex ratio (pvalue $<0.05$ ). Among mature individuals, females insignificantly outnumbered males 1:0.3 (p-value>0.05), and the significant majority of juveniles were females 1:0.15 (pvalue $<0.05$ ). 
Males ranged from 81 to $95.6 \mathrm{~cm} L_{\mathrm{T}}$, with three mature individuals encountered (37.8\% of males). Clasper size increased between 89.5 and $90.5 \mathrm{~cm} L_{\mathrm{T}}$ (Figure 33-a). The smallest mature measured $90.2 \mathrm{~cm} L_{\mathrm{T}}\left(94.4 \% L_{\mathrm{T} \max }\right)$, the largest immature male was 95 cm $L_{\mathrm{T}}$, with $L_{\mathrm{T} 50}$ estimated at $92.6 \mathrm{~cm} L_{\mathrm{T}}$. Females ranged from 69.5 to $123.3 \mathrm{~cm} L_{\mathrm{T}}$, with 9 mature (21.4\% of females) (Figure 33-b). Oviducal gland width spiked between 102 and $104 \mathrm{~cm} L_{\mathrm{T}}$ (Figure 33-c). The smallest mature female measured $105 \mathrm{~cm} L_{\mathrm{T}}$, the largest immature reached $111 \mathrm{~cm} L_{\mathrm{T}}$, and $L_{\mathrm{T} 50}$ was estimated at $105.9 \mathrm{~cm} L_{\mathrm{T}}$.

a.
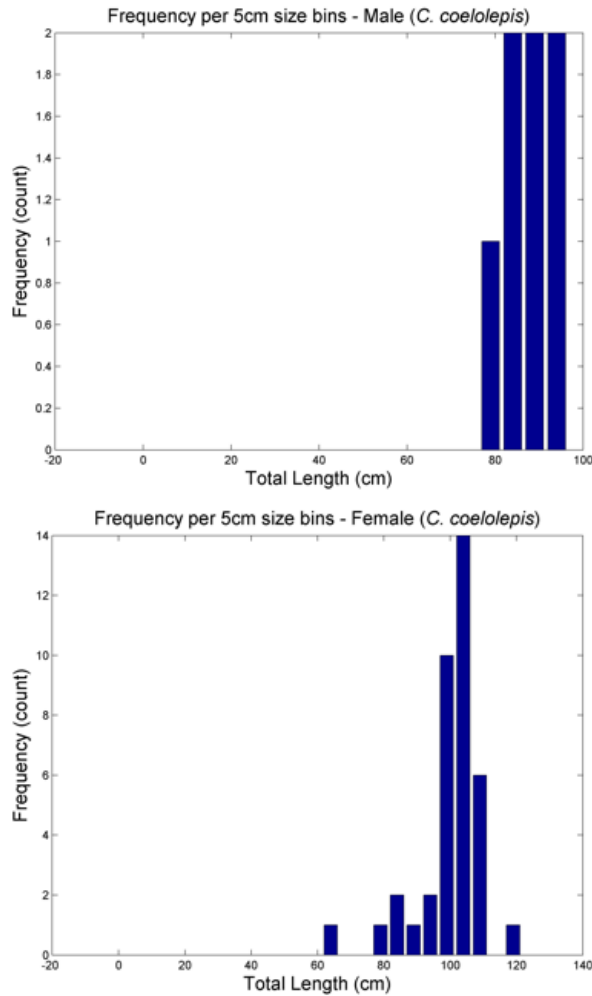

b.

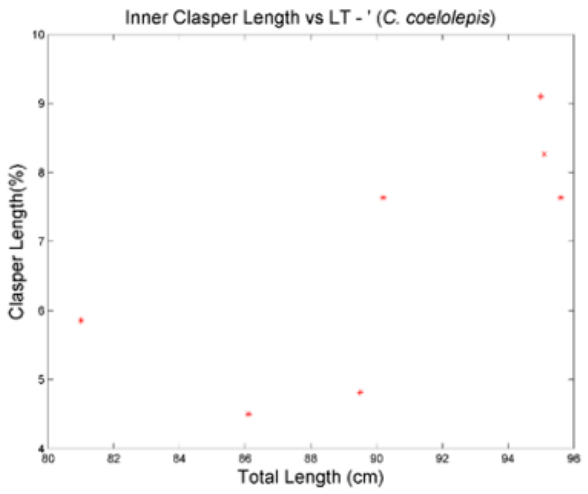

d.

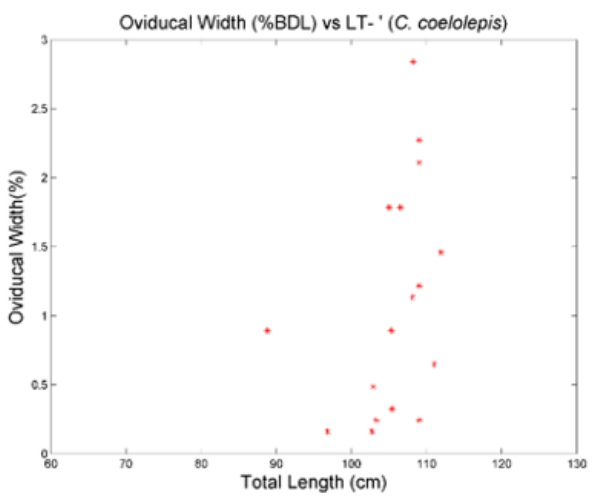

Figure 33. Centroscymnus coelolepis: a) relationship between inner clasper length (\%LT) and LT (males), b) size distribution of females, c) relationship between shell gland width (\%LT) and LT (females).

Females had between five and 22 eggs in their left uterus and between four and 20 in their right with an average of 22 eggs evenly distributed between their two uteri. The 
largest mature egg had a maximum diameter of $5.9 \mathrm{~cm}$ across. Oocytes were numerous with between 20 and 43 per ovary with an average of 24.9 per ovary. A single pregnant individual was examined from Walters Shoal and measured $107.9 \mathrm{~cm} L_{\mathrm{T}}$, bearing 12 pups (five left, seven right uterus), which averaged $70.9 \mathrm{~mm} L_{\mathrm{T}}$ and were undeveloped with a large portion of yolk un-absorbed.

Stomach contents included bony fish (two females, both $109 \mathrm{~cm} \mathrm{LT}$ ) cephalopod (two females 103 and $105 \mathrm{~cm} \mathrm{LT}$ ), and mammal (pinniped) remains (two females 103 and 109 $\mathrm{cm} \mathrm{LT).}$

Males and females were taken from the same seamounts along the Southwest Indian Ocean Ridge and Walters Shoal with a depth range of 89 to 1310 m (Figure 34).

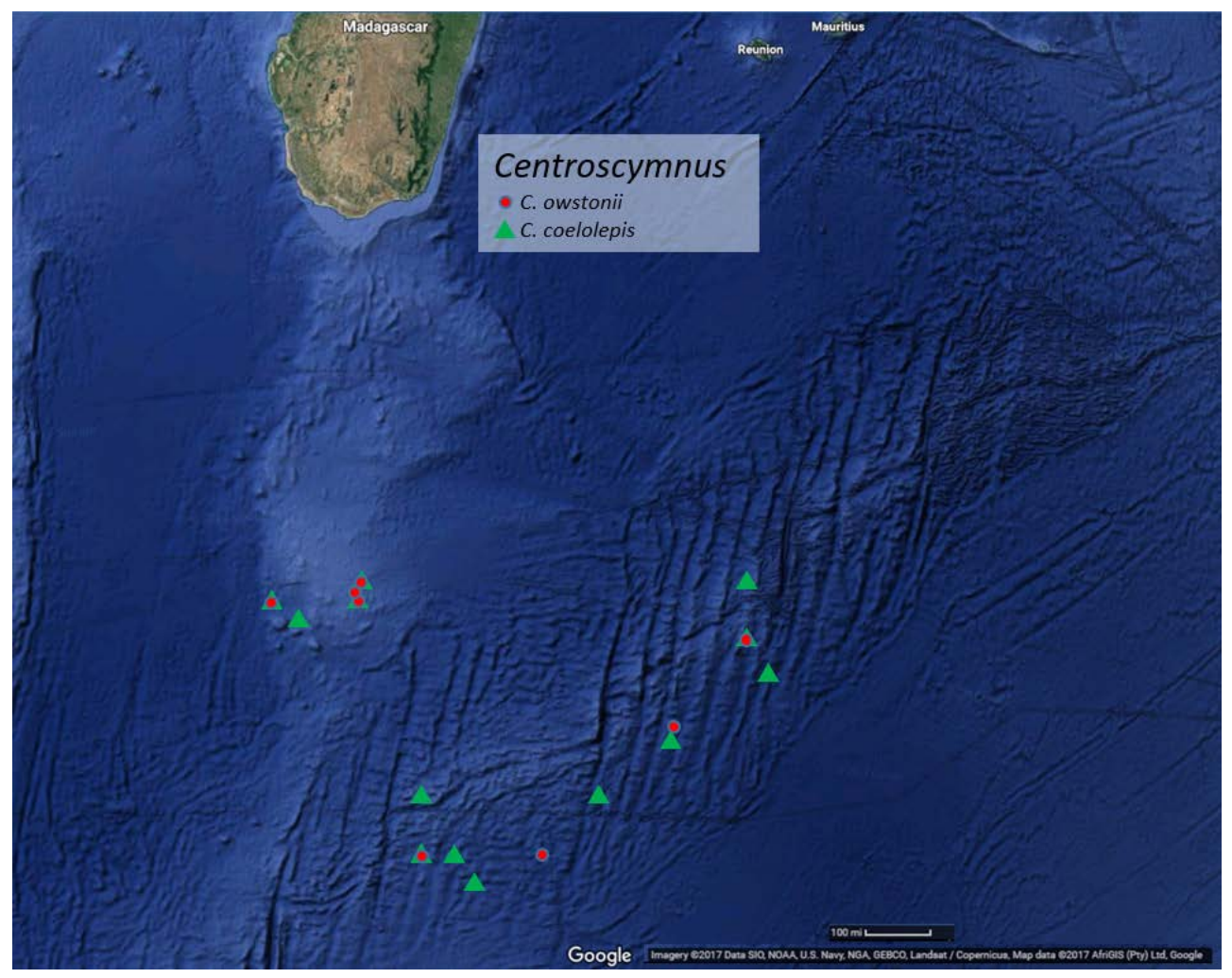

Figure 34. Distribution of the genus Centroscymnus: C. coelolepis (green triangle) and C. owstonii (red circle). Map data: Google, Image (C) 2017 DigitalGlobe. 
Centroscymnus owstonii, Garman, 1906, Roughskin Dogfish. In total, 44 C. owstonii (31 females and 13 males) were encountered, with a F:M ratio of roughly 1:0.4, significantly higher $(\mathrm{p}$-value $<0.05)$ than unity. Examination by maturity stage revealed adult females were more abundant than adult males 1:0.4 (p-value $<0.05)$.

Males ranged from 44.2 to $95.1 \mathrm{~cm} L_{\mathrm{T}}$, with 3 mature (23.1\% total) smallest mature measuring $84.9 \mathrm{~cm} L_{\mathrm{T}}, 89.3 \% L_{\mathrm{T}}$ max (Figure 35-a). Clasper length increased between 81 and $88 \mathrm{~cm} L_{\mathrm{T}}$ (Figure 35-b), the largest immature male measured $91 \mathrm{~cm} L_{\mathrm{T}}$, and $L_{\mathrm{T} 50}$ was estimated at $90 \mathrm{~cm} L_{\mathrm{T}}$. Females ranged from 75.6 to $114 \mathrm{~cm} L_{\mathrm{T}}$, with five mature (16.1\% of encountered) (Figure 35-c). Oviducal gland width increased between 108 and $110 \mathrm{~cm}$ $L_{\mathrm{T}}$ (Figure $35-\mathrm{d}$ ). The smallest mature measured $79.8 \mathrm{~cm} L_{\mathrm{T}}$ and was $70 \% L_{\mathrm{Tmax}}$, the largest immature female was $113 \mathrm{~cm} L_{\mathrm{T}}$, and $L_{\mathrm{T}} 50$ was $99.9 \mathrm{~cm} L_{\mathrm{T}}$. 
a.

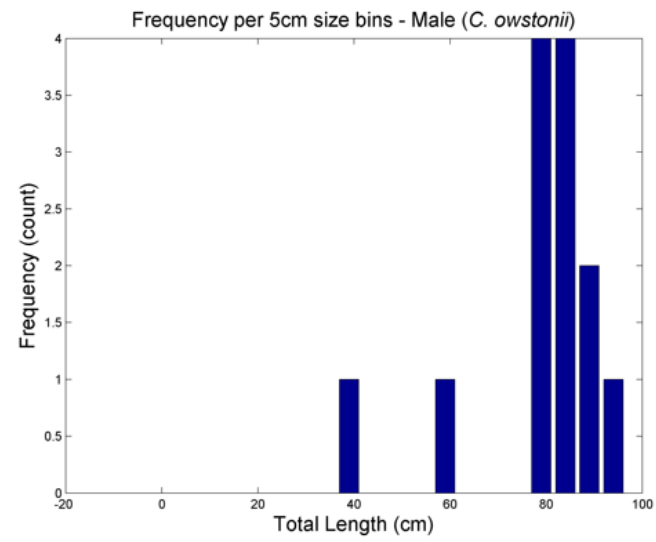

c.

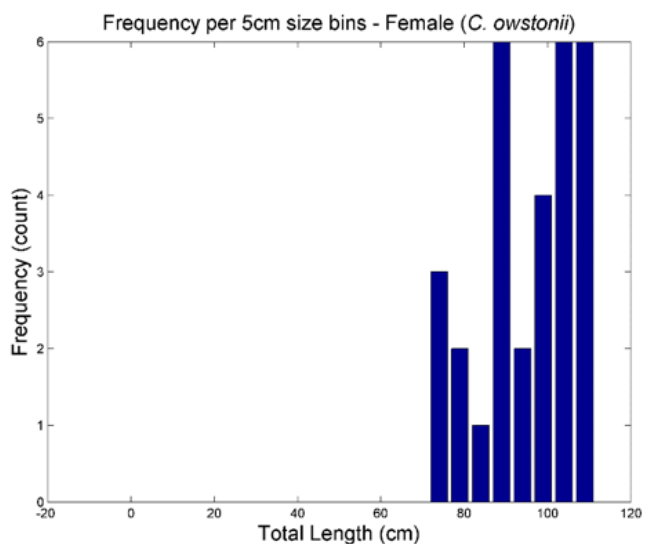

Inner Clasper Length vs LT - ' (C. owstonii)

b.
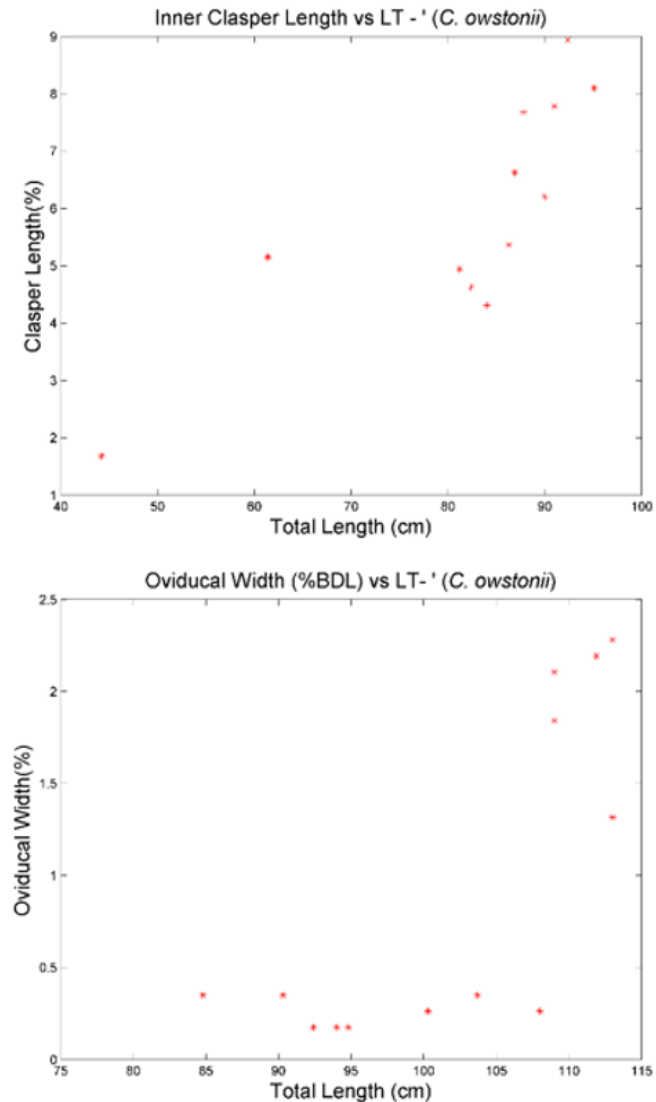

Figure 35. Centroscymnus owstonii: a) size distribution of males, b) relationship between inner clasper length (\%LT) and LT (males), c) size distribution of females, d) relationship between shell gland width (\%LT) and LT (females).

Females had between four and 15 eggs in their left uterus and between six and 14 in their right, with the largest mature egg reaching a maximum diameter of $6.2 \mathrm{~cm}$ across.

Diet was composed of cephalopods in one male, bony fishes in two females, and a combination of cephalopod (75\%) and fish (25\%) in one female.

This species was found at 800 to $1400 \mathrm{~m}$ deep at both the Southwest Indian Ocean Ridge and Walters Shoal in between 686 and 1350 meters, with its distribution overlapping that of Centroscymnus coelolepis (Figure 34). 
Centroselachus crepidater (Barbosa du Bocage \& de Brito Capello, 1864), Longnose Velvet Dogfish. In total, 300 C. crepidater (217 females and 83 males) were examined, with an overall F:M ratio of 1:0.4, significantly different from the expected equal ratio (pvalue $<0.05$ ). Examination by maturity status revealed mature females outnumbered mature males by nearly threefold, F:M 1:0.4 (p-value<0.05), and juvenile female outnumbered juvenile males 1:0.2 ( $\mathrm{p}$-value $<0.05)$.

Males ranged from 20.5 to $94.9 \mathrm{~cm} L_{\mathrm{T}}$, with 54 mature (65.1\% of those encountered) (Figure 36-a). Clasper length increased between 60 and $63 \mathrm{~cm} L_{\mathrm{T}}$ (Figure 36-b) with the smallest mature individual measuring $63.2 \mathrm{~cm} L_{\mathrm{T}}\left(66.6 \% L_{\mathrm{T} \max }\right.$ ), the largest immature measured $66.4 \mathrm{~cm} L_{\mathrm{T}}$, and $L_{\mathrm{T} 50}$ was estimated to be $60.4 \mathrm{~cm} L_{\mathrm{T}}$. Females ranged from 37.5 to $145.8 \mathrm{~cm} L_{\mathrm{T}}$, with 93 mature (42.9\% of total females) (Figure 36-c). Oviducal gland width increased sharply between 73 and $75 \mathrm{~cm} L_{\mathrm{T}}$ (Figure 36-d), with the smallest mature measuring $78 \mathrm{~cm} L_{\mathrm{T}}\left(53.5 \% L_{\mathrm{T} \max }\right)$, the largest immature $86.0 \mathrm{~cm} L_{\mathrm{T}}$, and $L_{\mathrm{T} 50}$ was estimate at $88.3 \mathrm{~cm} L_{\mathrm{T}}$. 

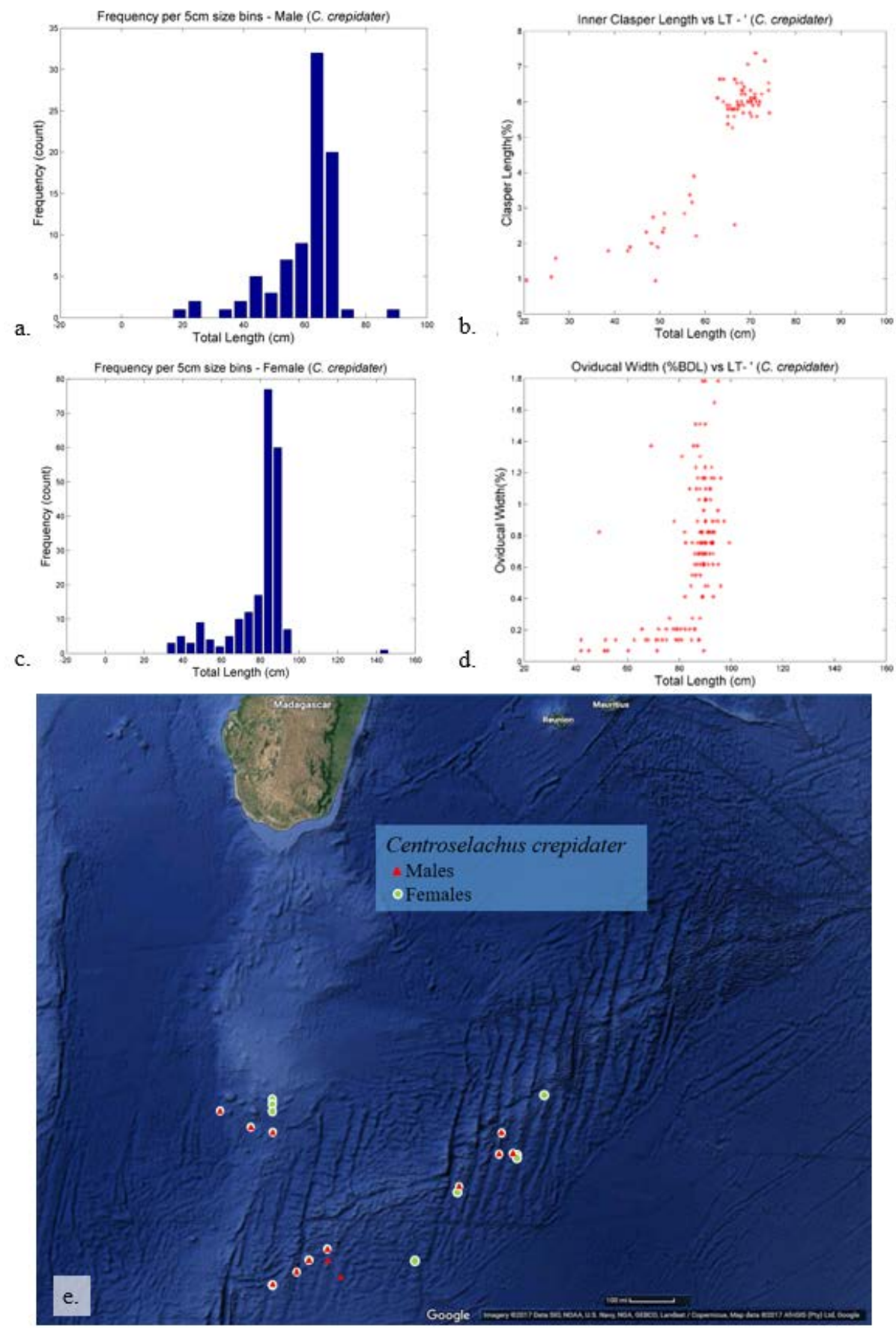

Figure 36. Centroselachus crepidater: a) size distribution of males, b) relationship between inner clasper length (\%LT) and LT (males), c) size distribution of females, d) relationship between shell gland width (\%LT) and LT (females), and e) distribution of males (red triangles) and females (green circles). Map data: Google, Image (c) 2017 DigitalGlobe. 
Females had an average of eight mature eggs between two uteri, with between one and 16 per uterus, measuring $3.5 \mathrm{~cm}$ across. Oocytes were numerous, up to 24 per ovary. Four pregnant females were dissected for fecundity data. Mothers ranged from 89 to 94 cm $L_{\mathrm{T}}$ with an average $L_{\mathrm{T}}$ of $92.2 \mathrm{~cm}$. Number of pups ranged from two to seven with an average of 3.8 per mother.

Diet was found in 30 individuals and contained fishes in 26 specimens (86.7\%), cephalopod in one specimen (3.3\%), crustacean in one specimen, and mammal (pinniped) in one specimen. One individual had both crustacean (33\% by volume) and fish (64\%), and another had cephalopod and fish (50\%). There was no evidence of correlation between sex, length, or maturity stage and diet.

Females (adult and subadult) were taken from both the Southwest Indian Ocean Ridge and Walters Shoal. Males and pregnant females were only encountered along the Southwest Indian Ocean Ridge (Figure 36-e). This species had a depth range of 89 to $1365 \mathrm{~m}$. Depth did not appear to correlate with sex, or maturity status.

Zameus squamulosus (Günther, 1877), Velvet Dogfish. Five Zameus squamulosus (one female and four males) were encountered, but the sample size was too small to be of significance (p-value >0.05). Males ranged from 52 to $53 \mathrm{~cm} L_{\mathrm{T}}$, with three mature individuals. Clasper length increased between 52 and $53 \mathrm{~cm} L_{\mathrm{T}}$, with the smallest mature measuring $52.5 \mathrm{~cm} L_{\mathrm{T}}\left(99.1 \% L_{\mathrm{T} \max }\right)$, and the only immature measured $52 \mathrm{~cm} L_{\mathrm{T}}, L_{\mathrm{T} 50}$ was estimated to occur at $52.3 \mathrm{~cm}$ length. The only female specimen measured $92.9 \mathrm{~cm}$ $L_{\mathrm{T}}$, nearly twice the size of the largest male, and was pregnant. Oviducal gland width was $1.0 \mathrm{~cm}$, and the female had three pups in left uterus. Pups were all female, measured from 
$14.8 \mathrm{~cm}$ to $15.5 \mathrm{~cm}$, and were moderately developed with a large amount of external yolk sack not yet absorbed.

This species was encountered on Walters Shoal with a depth range of 810 to $1060 \mathrm{~m}$, and was collected in low numbers (one or two) from different seamounts (Figure 32).

Somniosus cf rostratus, Little Sleeper Shark. A single specimen of S. cf. rostratus was collected, a neonate male measuring $30.2 \mathrm{~cm} L_{\mathrm{T}}$, and taken from the Southwest Indian Ocean Ridge between 670 and 755 m deep (Figure 32).

Dalatias licha (Bonnaterre, 1788), Kitefin Shark. In total, 175 D. licha (165 females and 10 males) were collected with a F:M ratio of 1:0.1, significantly different from the expected equal ratio ( $\mathrm{p}$-value $<0.05$ ). Comparison by maturity stage revealed females outnumbered males in adult stage by 1:0.05 ( $\mathrm{p}$-value $<0.05)$ and in juvenile stage by 1:0.07 (p-value $<0.05)$.

Males ranged in size from 47.1 to $112 \mathrm{~cm} L_{\mathrm{T}}$, with 4 mature ( $40 \%$ of males) (Figure 37-a). Clasper length increased between 103 and $107 \mathrm{~cm} L_{\mathrm{T}}$ (Figure 37-b), with the smallest mature occurring at $107 \mathrm{~cm} L_{\mathrm{T}}\left(95.5 \% L_{\mathrm{T} \max }\right)$, largest immature at $103 \mathrm{~cm}$ $L_{\mathrm{T}}$, and $L_{\mathrm{T} 50}$ estimated to be $105.0 \mathrm{~cm} L_{\mathrm{T}}$. The smallest free-swimming male, measuring $47.1 \mathrm{~cm}$ TL bore an umbilical scar indicating a minimum size for the species. Females ranged from 56 to $157.3 \mathrm{~cm} L_{\mathrm{T}}$, with 76 mature (46.1\% females encountered) (Figure 37c). Oviducal gland width increased between $122 \mathrm{~cm} L_{\mathrm{T}}$ and $134 \mathrm{~cm} L_{\mathrm{T}}$ (Figure 37-d). Smallest mature measured $113.5 \mathrm{~cm} L_{\mathrm{T}}\left(72.2 \% L_{\mathrm{T} \max }\right)$, the largest immature at $147 \mathrm{~cm} L_{\mathrm{T}}$, and $L_{\mathrm{T} 50}$ estimated to be $130.0 \mathrm{~cm} L_{\mathrm{T}}$. 
Females had up to 58 undeveloped oocytes in a single ovary with an average of 15 mature oocytes between both ovaries. Mature eggs averaged seven per uterus, although there could be as many as 26 eggs in a single uterus and 35 between both uteri. Mature eggs measured up to $9.2 \mathrm{~cm}$ across. 

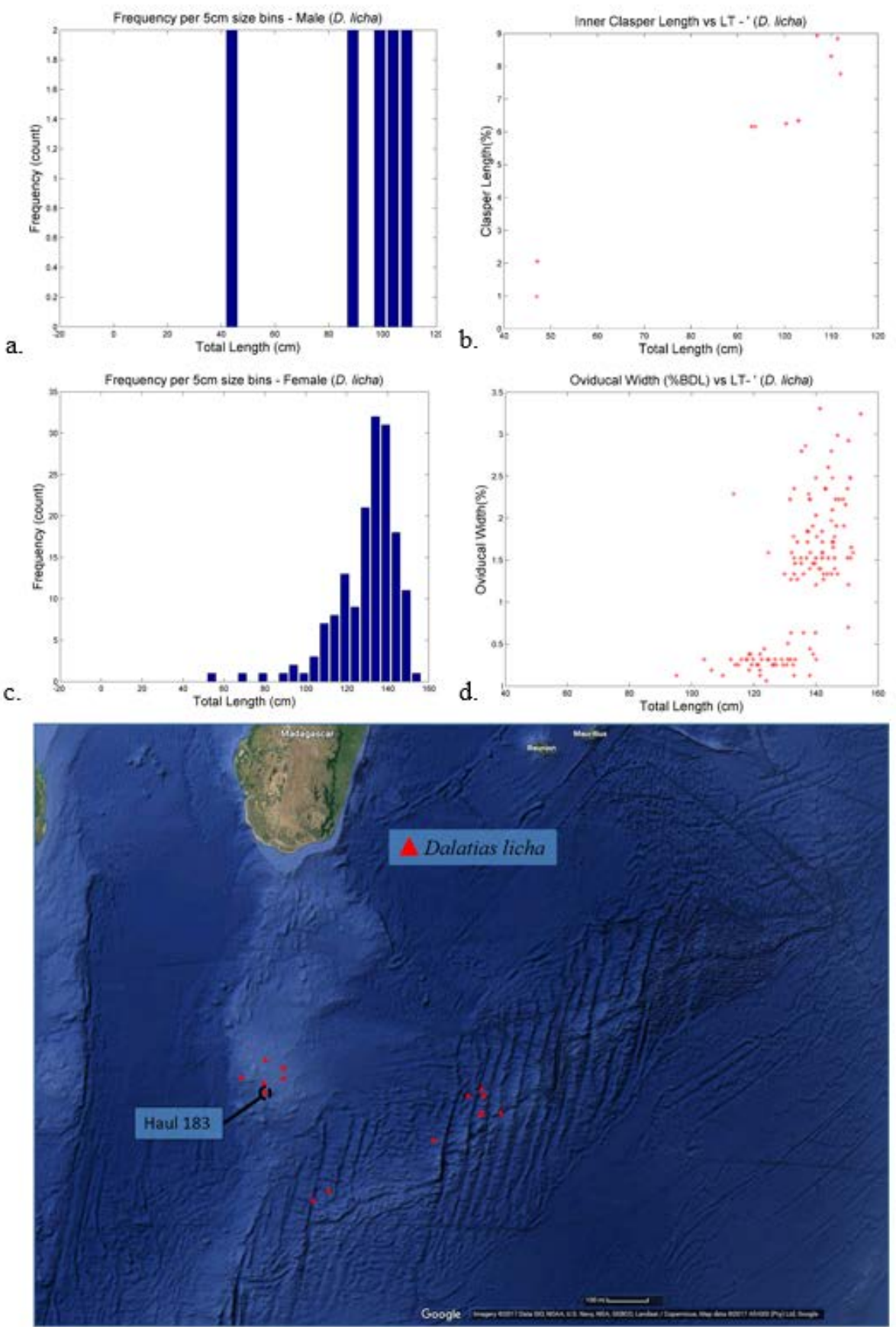

e.

Figure 37. Dalatias licha: a) size distribution of males, b) relationship between inner clasper length (\%LT) and LT (males), c) size distribution of females, d) relationship between shell gland width (\%LT) and LT (females), and e) distribution (red triangles) and Haul 183 (indicated). Map data: Google, Image (C) 2017 DigitalGlobe. 
Diet was examined in 65 specimens, and mainly consisted of bony fishes with 50 (76.9\%) individuals containing only fish, three (4.6\%) squid, three (4.6\%) shark, and one (1.5\%) of invertebrates. Mixed diet was found in 8 (12.3\%) individuals and contained of average of $19 \%$ shark, $35 \%$ squid, and $46 \%$ squid by volume. There was no correlation between sex, length, or maturity and diet composition.

Males were found exclusively on the Southwest Indian Ocean Ridge with a depth range of 580 to $1290 \mathrm{~m}$. Females were most commonly encountered along Walters Shoal, between 460 to $1311 \mathrm{~m}$ deep, with only reproductively inactive females found at the Southwest Indian Ocean Ridge (Figure 37-e). Individuals were normally taken in low numbers (one to three per haul), with the exception of a single haul on Walters Shoal, fishing depths between 560 and $1007 \mathrm{~m}$, in which 75 females were collected (haul 183). The females in this haul had a maturity ratio equal to the rest of the survey. No males were present in this haul, and no deviation in diet (quantity or composition) was apparent. Apristurus sinensis, Chu and Hu, 1981, South China Catshark. In total, 93 total A. sinensis (34 females and 59 males) were encountered, with a F:M ratio of 1:1.7, significantly favoring males (p-value $<0.05$ ). Males ranged from 32.2 to $102.5 \mathrm{~cm} L_{\mathrm{T}}$, with 39 mature (66.1\% total males) (Figure 38-a). Clasper length increased sharply between 55 and $60 \mathrm{~cm} L_{\mathrm{T}}$ (Figure 38-b), with the smallest mature measuring $63.1 \mathrm{~cm} L_{\mathrm{T}}$ (61.6\% $L_{\mathrm{T} \max }$ ), the largest immature $66 \mathrm{~cm} L_{\mathrm{T}}$, and $L_{\mathrm{T} 50}$ estimated to be $60.3 \mathrm{~cm} L_{\mathrm{T}}$. Females ranged from 47.7 to $109.1 \mathrm{~cm} L_{\mathrm{T}}$ (Figure 38-c). Two female specimens were examined internally; one was determined to be immature at $74.4 \mathrm{~cm} L_{\mathrm{T}}$ while the other was mature at $93.9 \mathrm{~cm} L_{\mathrm{T}}$. 
Table 9b. A list of species encountered, the relationship between total length $\left(L_{\mathrm{T}}\right)$ and length at first maturity, length at first maturity in relation to maximum length $\left(L_{\mathrm{T} \max }\right)$, and length at 50\% maturity ( $\left.L_{T 50}\right)$ for Carcharhiniformes: scyliorhinids, Pseudotriakidae, and Holocephali: Chimaeridae.

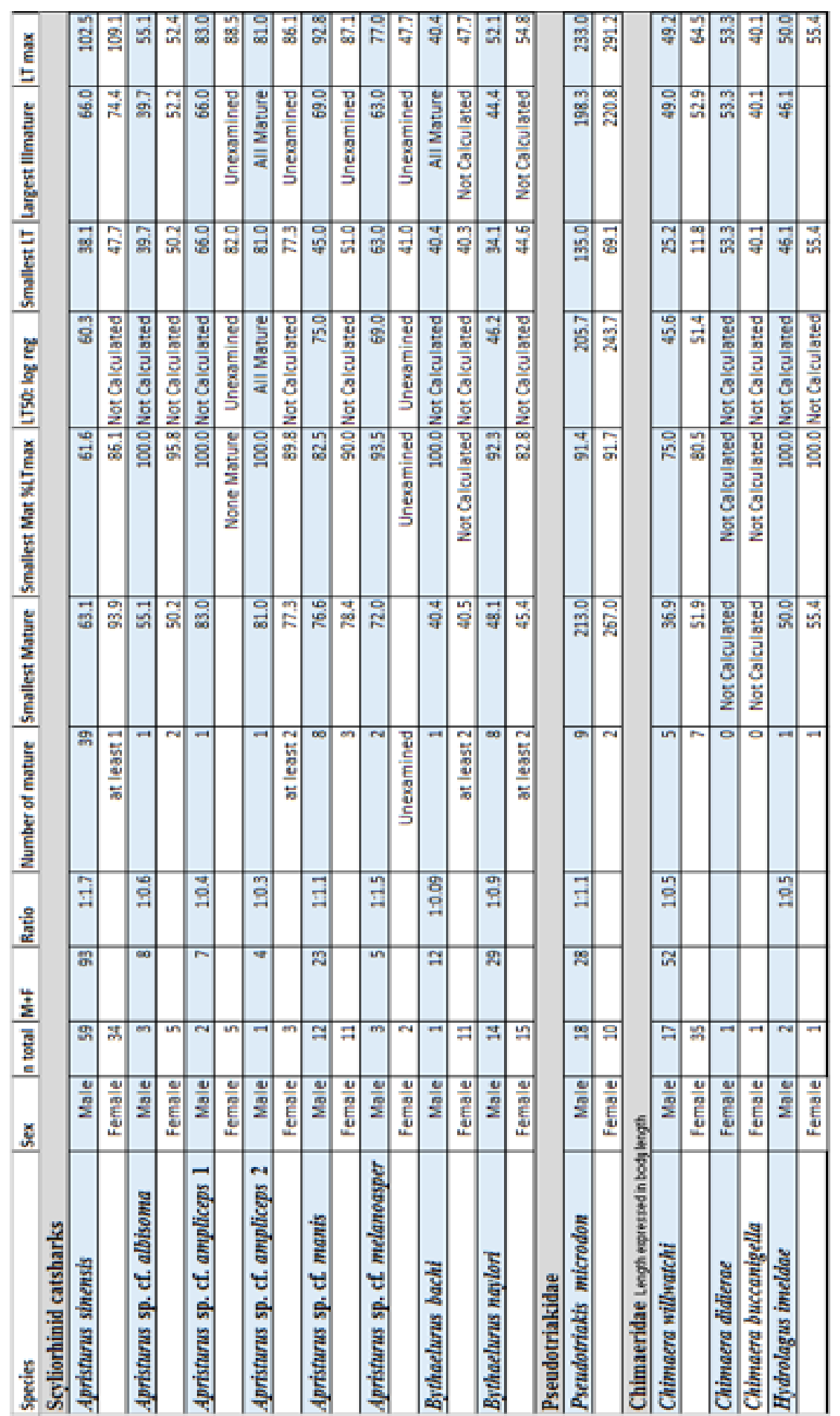



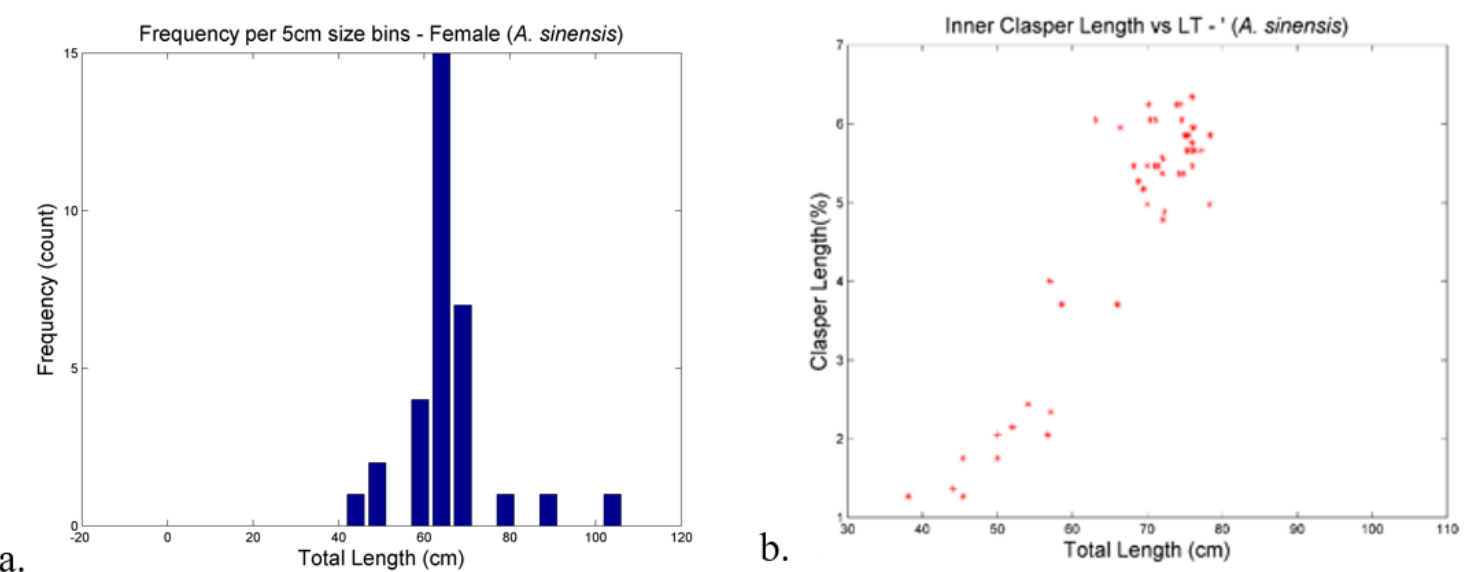

a.

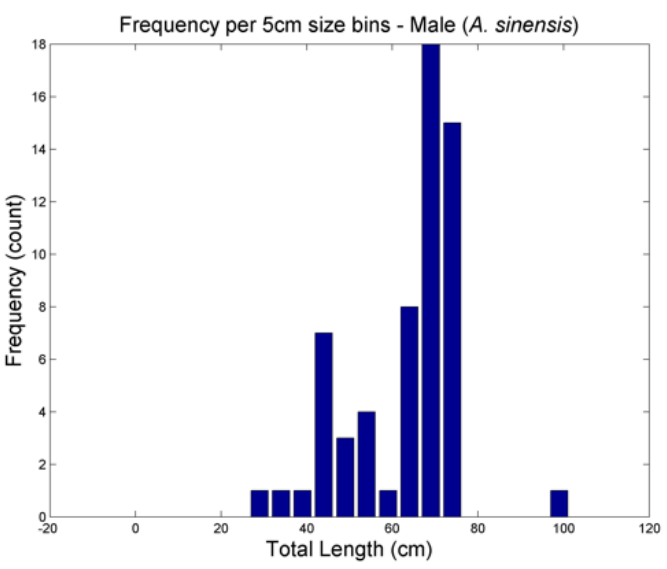

Figure 38. Apristurus sinensis: a) size distribution of males, b) relationship between inner clasper length (\%LT) and LT (males), c) size distribution of females.

This species was by far the most commonly encountered catshark in the area and appears to be widespread, with males and females taken from the same seamounts, 800 to 1300 m deep, from both the Southwest Indian Ocean Ridge and Walters Shoal, between 89 and $1365 \mathrm{~m}$ deep (Figure 39). 


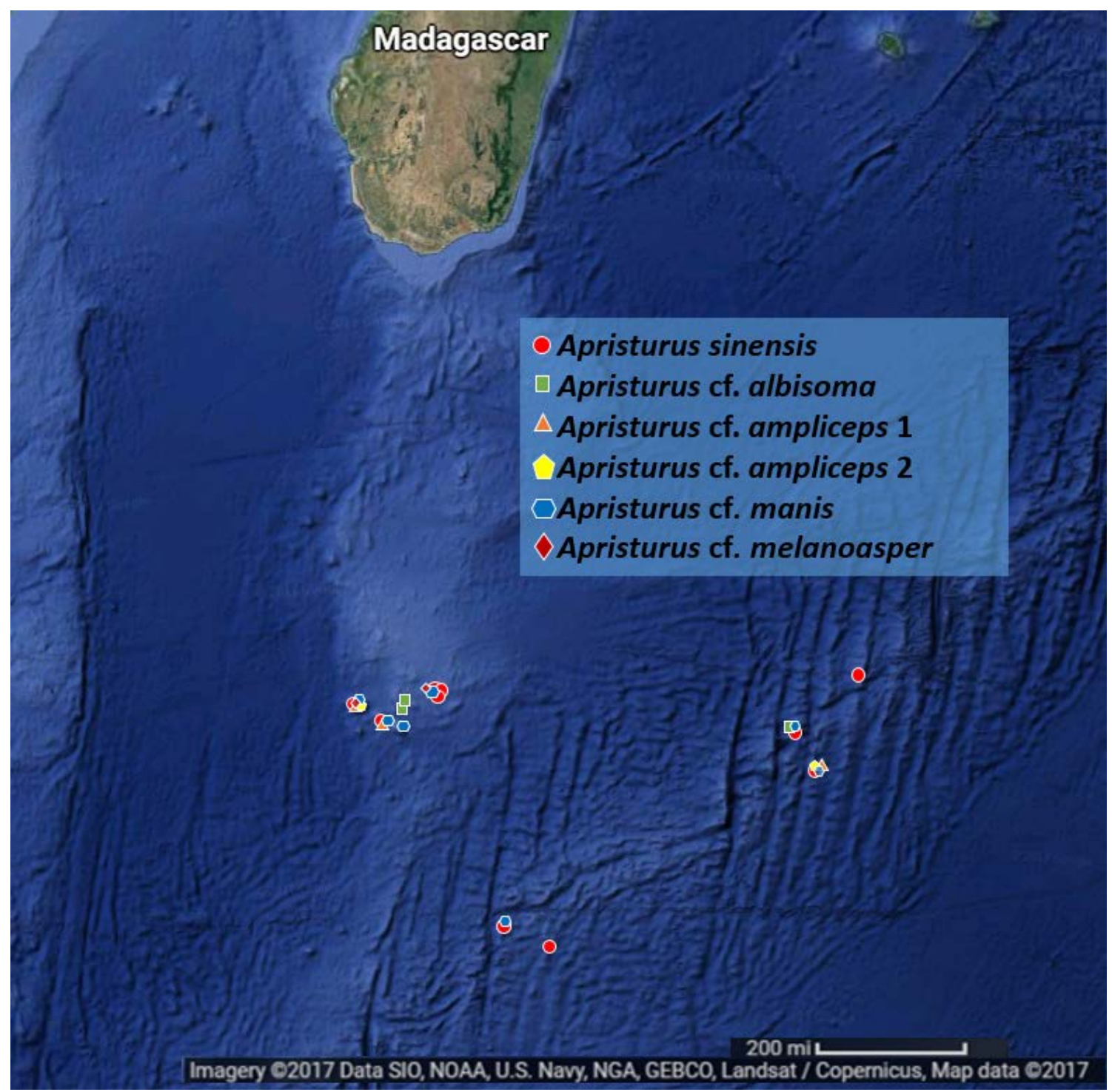

Figure 39. Distribution of the genus Apristurus: A. sinensis (red circle), A. cf. albisoma (green square), A. cf. ampliceps 1 (orange triangle), A. cf. ampliceps 2 (yellow pentagon), A. cf. manis (blue hexagon), and A. cf. melanoasper (maroon diamond). Map data: Google, Image (C) 2017 DigitalGlobe.

Apristurus cf. albisoma, White-Bodied Catshark. In total, eight specimens of $A$. cf. albisoma were encountered (five females and three males), with an overall F:M ratio of 1:0.6 with females not significantly outnumbering males (p-value $>0.05$ ). Males ranged 
from 39.7 to $55.1 \mathrm{~cm} L_{\mathrm{T}}$, with only the largest being mature. Females ranged from 50.2 to $52.4 \mathrm{~cm} L_{\mathrm{T}}$, and were not examined internally.

This species was relatively uncommon, and found only at one site on the Southwest Indian Ocean Ridge and one site on the Madagascar Ridge between 890 and 1300 meters (Figure 39).

Apristurus cf. ampliceps 1, Roughskin Catshark. In all, seven A. cf. ampliceps 1 were collected (five females and two males), with a F:M ratio of 1:0.4, not significantly favoring females ( $p$-value $>0.05$ ).

Males measured 66 and $83 \mathrm{~cm} L_{\mathrm{T}}$, the larger of the two was mature. Females ranged from 82 to $88.5 \mathrm{~cm} L_{\mathrm{T}}$ and were not examined internally. Mature males and females of this species were scarred all over their bodies with bite marks matching the teeth of their own species.

Males and females were taken from Walters Shoal between 1000 and $1300 \mathrm{~m}$ deep, with two females collected from two relatively close sites along the Southwest Indian Ocean Ridge, 1200 to 1400 m deep (Figure 39).

Apristurus cf. ampliceps 2, Roughskin Catshark. A total of four A. cf. ampliceps 2 were taken (three females and one male), a sex ratio of 1:0.3, not significantly favoring females (p-value $>0.05)$.

The lone male was mature at $81 \mathrm{~cm} L_{\mathrm{T}}$. Females ranged from 77.3 to $86.1 \mathrm{~cm} L_{\mathrm{T}}$, and were not examined internally. This species was scarred in a way that matched $A$. cf. ampliceps 1. 
The male and females were encountered together on Walters Shoal, 1000 to $1100 \mathrm{~m}$ deep, and a single female was collected from the Southwest Indian Ocean Ridge, 1200 to $1300 \mathrm{~m}$ deep (Figure 39).

Apristurus cf. manis, Ghost Catshark. A total of 23 A. cf. manis (11 females and 12 males) were collected with a F:M sex ratio of 1:1.1, not significantly different (pvalue $>0.05$ ) from unity. Males ranged from 45 to $92.8 \mathrm{~cm} L \mathrm{~T}$, with 8 mature $(66.7 \%$ of encountered) (Figure 40-a). Clasper size increased between 67 and $76 \mathrm{~cm} L_{\mathrm{T}}$ (Figure 40b), with the smallest mature measuring $76.6 \mathrm{~cm} L_{\mathrm{T}}\left(82.5 \% L_{\mathrm{T} \max }\right)$, the largest immature measuring $69 \mathrm{~cm} L_{\mathrm{T}}$, and $L_{\mathrm{T} 50}$ estimated at $75 \mathrm{~cm} L_{\mathrm{T}}$. Females ranged from 78.4 to 87.1 $\mathrm{cm} L_{\mathrm{T}}$, with at least 3 mature (Figure $40-\mathrm{c}$ ). Of the females examined internally, the smallest mature measured $78.4 \mathrm{~cm} L_{\mathrm{T}}\left(90.0 \% L_{\mathrm{T} \max }\right)$.

Males and females of this species were covered in scars matching the teeth of their own species. The absence of these scars in reproductively inactive specimens suggests that this species uses its teeth to position during copulation. 

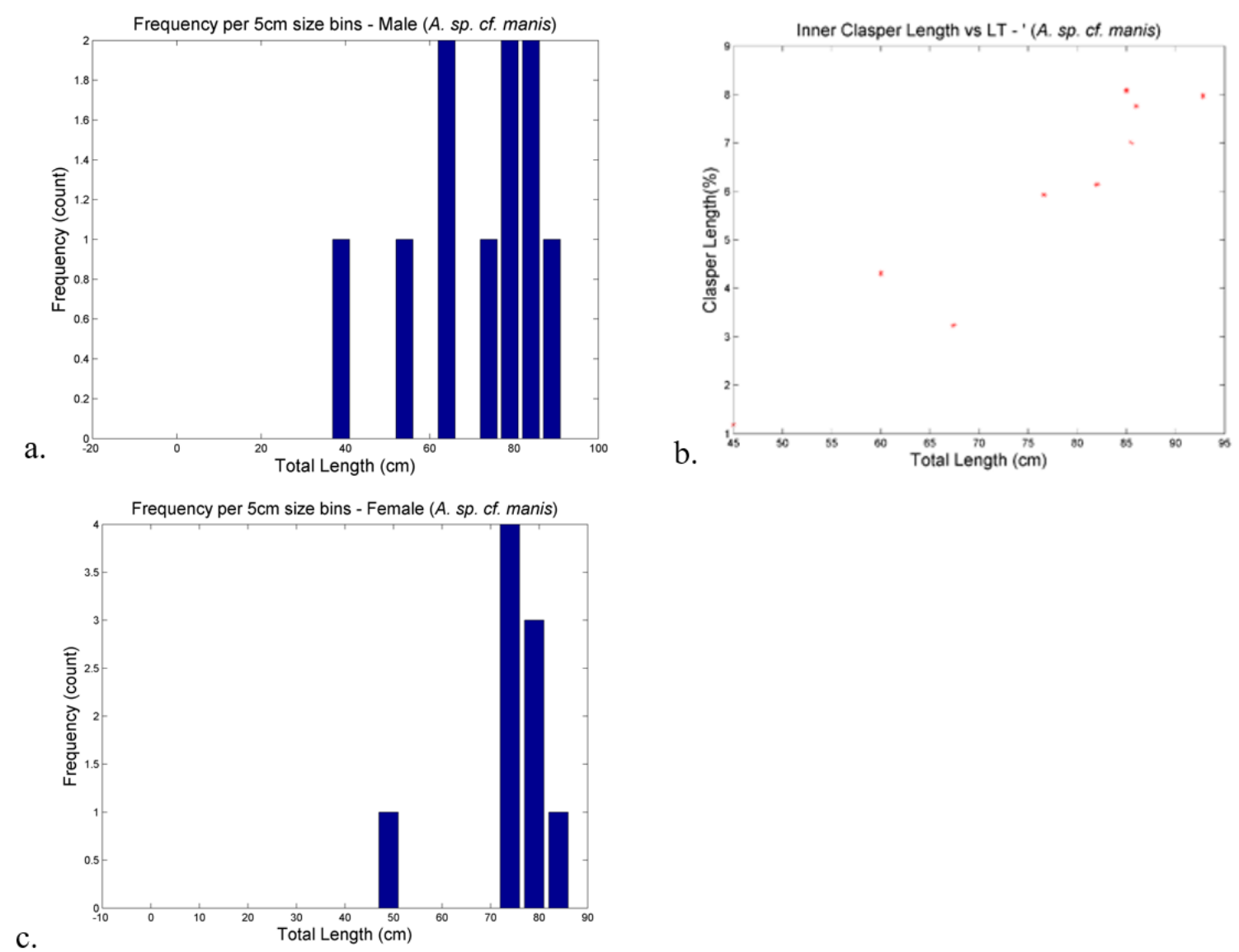

Figure 40. Apristurus cf. manis: a) size distribution of males, b) relationship between inner clasper length (\%LT) and LT (males), c) size distribution of females.

Females were encountered from the Southwest Indian Ocean Ridge and Walters Shoal between 620 and 1340 m deep, while males were found mostly on Walters Shoal between 1128 and 1270 m deep with two males from the Southwest Indian Ocean Ridge between 760 and $1250 \mathrm{~m}$ deep (Figure 39).

Apristurus cf. melanoasper, Black Roughscale Catshark. In all, five A. cf. melanoasper were collected, (two females and three males), with a sex ratio of 1:1.5, not significantly different from unity (p-value $>0.05$ ). Males ranged from 63 to $77 \mathrm{~cm} L_{\mathrm{T}}$, with 2 mature. Males were immature at $63 \mathrm{~cm} L_{\mathrm{T}}$, mature at $72 \mathrm{~cm} L_{\mathrm{T}}$ (93.5\% $L_{\mathrm{T} \max }$ ), $L_{\mathrm{T} 50}$ 
was estimated at $69 \mathrm{~cm} L_{\mathrm{T}}$. Females measured 41 and $47.7 \mathrm{~cm} L_{\mathrm{T}}$, and were not internally evaluated for maturity.

Specimens of this species were collected in separate hauls from five different sites on Walters Shoal with a depth range of 950 to $1340 \mathrm{~m}$ (Figure 39).

Bythaelurus bachi, Weigmann, Ebert, Clerkin, Stehmann, and Naylor, 2016, Bach's Catshark. A total of 12 B. bachi were encountered (11 females and one male), females significantly outnumbered males 1:0.09 (p-value <0.05). The only male collected was mature at $40.4 \mathrm{~cm} L_{\mathrm{T}}$. Females range from 40.3 to $47.7 \mathrm{~cm} L_{\mathrm{T}}$ (Figure $41-\mathrm{a}$ ). The smallest female was mature with large yolky oocytes, but lacked egg capsules. Two females, 40.5 and $40.8 \mathrm{~cm} L_{\mathrm{T}}$, were dissected and found to contain a single fully developed egg case in each uterus (Figure 41-b).

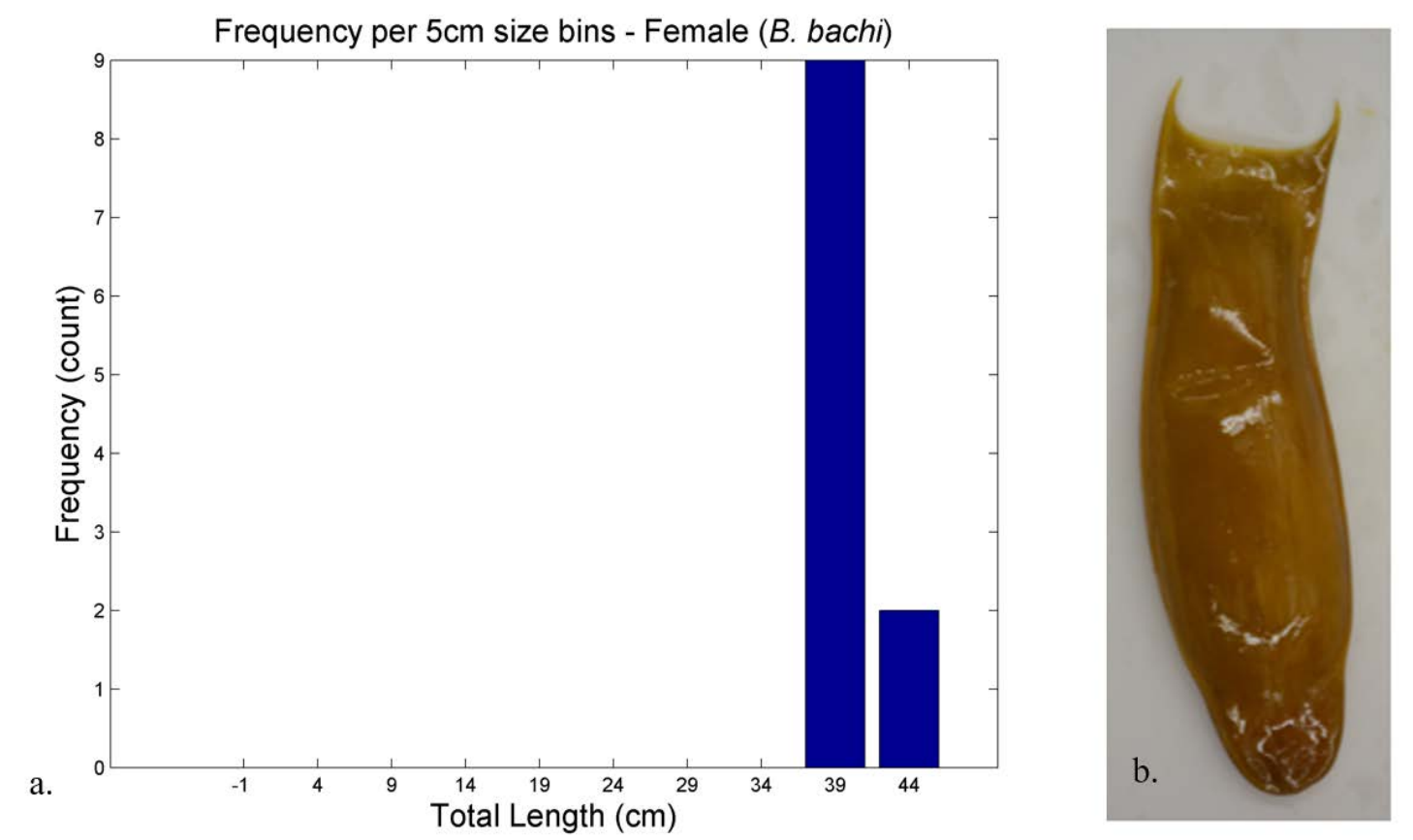

Figure 41. Bythaelurus bachi: a) size distribution of males, b) egg case removed from a non-type female. Photo by P.J. Clerkin. 
Egg cases were small, 62.2-66.9 mm long, broad, case width $24.2-27.9 \%$ case length, and thick, case height $16.7-17.7 \%$ case length; tan-brown in color with very fine striations, smooth to the touch. Lateral flanges of case narrow, about 1.3-1.6 mm wide, flat, and without T-shaped lateral surface, extending length of the egg case. The anterior border of case concave, with horns narrow, very short, and curved inwards. The posterior border lacked apparent horns.

This species was found from only two seamounts 35 miles apart on Walters Shoal between 800 and $1365 \mathrm{~m}$ deep (Figure 42).

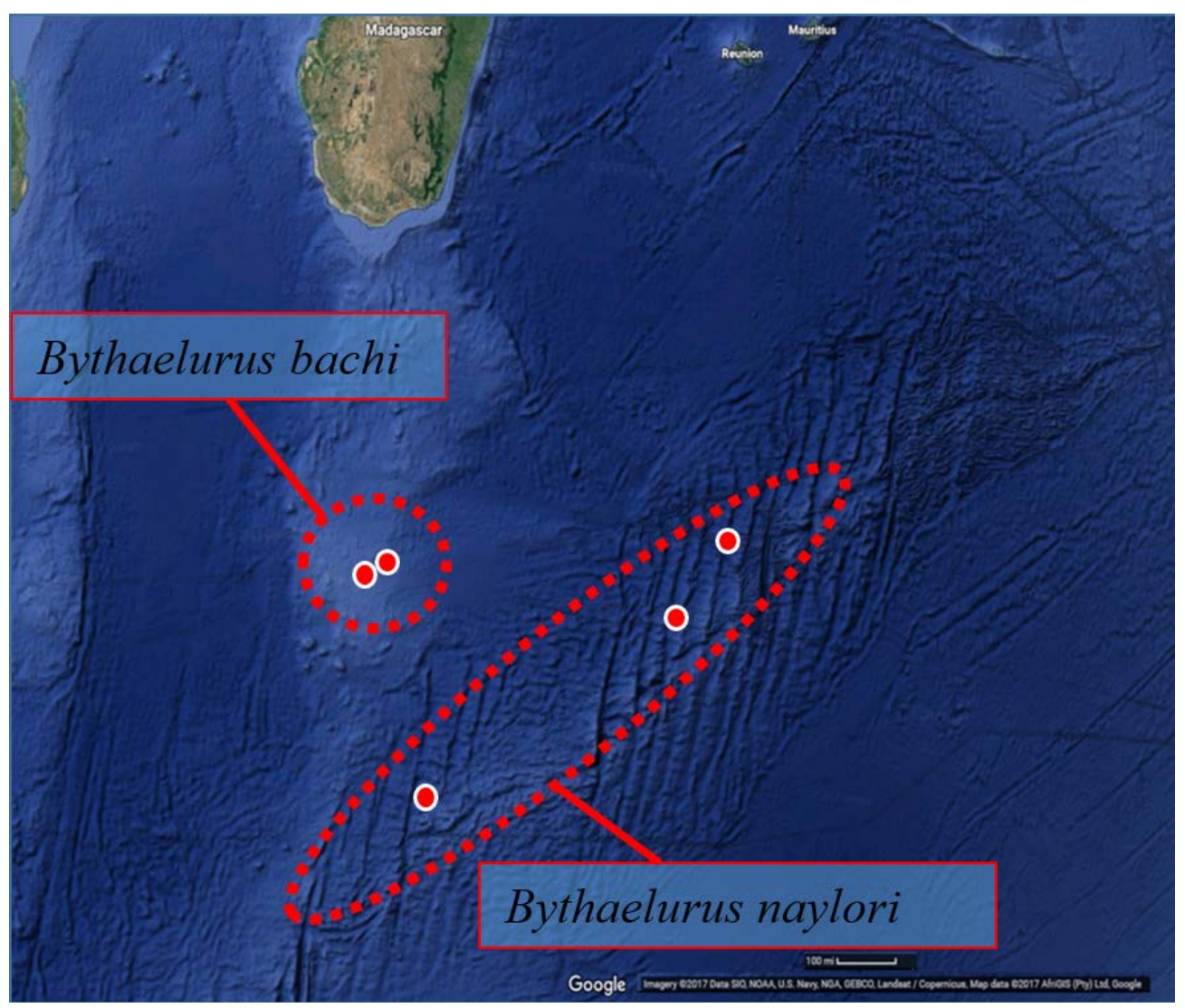

Figure 42. Distribution of the genus Bythaelurus: B. bachi (Walters Shoal) and B. naylori (Southwest Indian Ocean Ridge). Map data: Google, Image (C) 2017 DigitalGlobe. 
Bythaelurus naylori, Ebert and Clerkin, 2015, Dusky Snout Catshark. In all, 29 specimens of B. naylori (15 females, 14 males) were collected with a F:M sex ratio of 1:0.9, not significantly different from the expected even ratio ( $\mathrm{p}$-value $>0.05$ ). Males ranged from 34.1 to $52.1 \mathrm{~cm} L_{\mathrm{T}}$, with 8 mature (57.1\% of males) (Figure 43-a). Clasper length increased between 44 and $48 \mathrm{~cm} L_{\mathrm{T}}$ (Figure 43-b). The smallest mature measured 48.1 (92.3\% $\left.L_{\mathrm{T} \max }\right)$, the largest immature was $44.4 \mathrm{~cm} L_{\mathrm{T}}$, and $L_{\mathrm{T} 50}$ was estimated to be $46.2 \mathrm{~cm} L_{\mathrm{T}}$.

a.

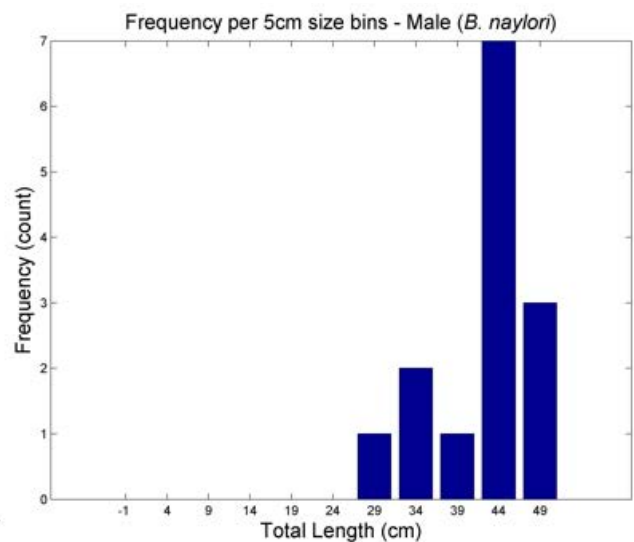

c.

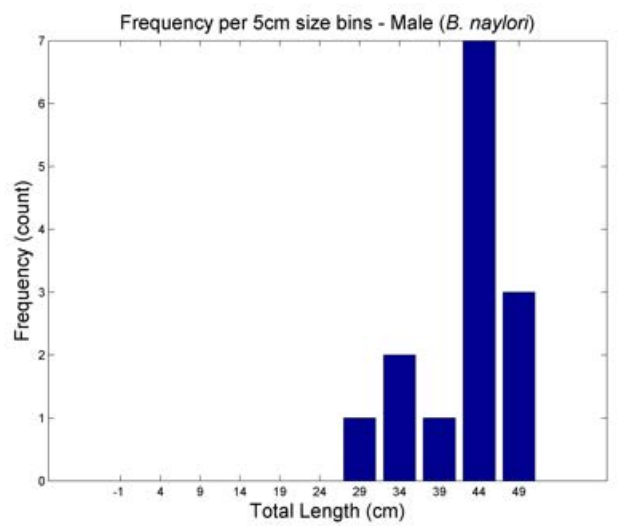

Inner Clasper Length vs LT - ' (B. naylori)
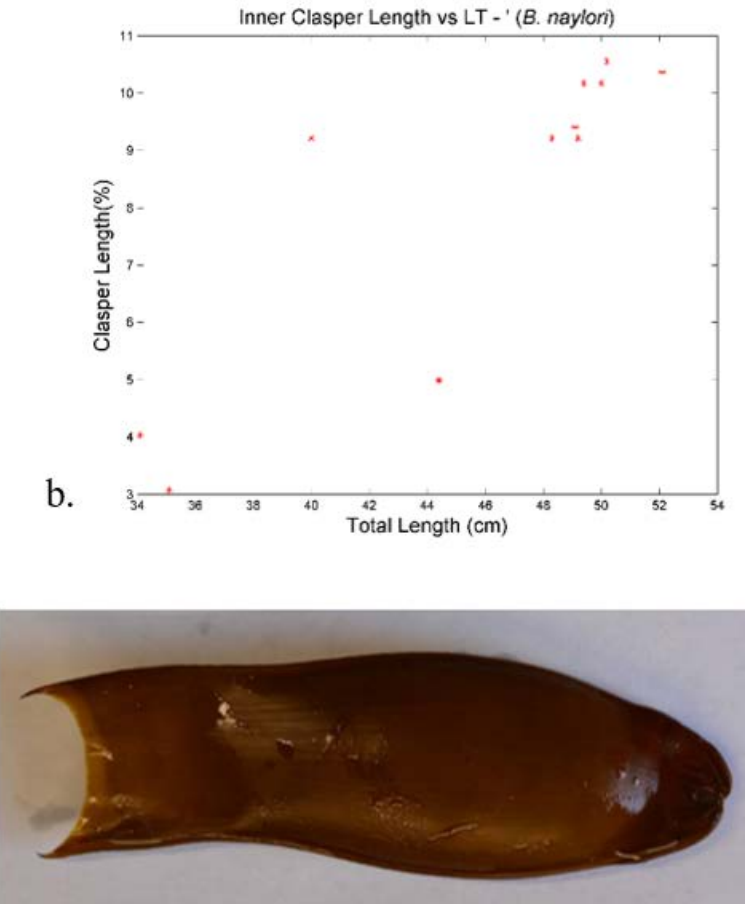

d.

Figure 43. Bythaelurus naylori: a) size distribution of males, b) relationship between inner clasper length (\%LT) and LT (males), c) size distribution of females, and d) egg case removed from a non-type female $452 \mathrm{~mm}$ TL. Photo by P.J. Clerkin.

Females ranged from 44.6 to $54.8 \mathrm{~cm} L_{\mathrm{T}}$ (Figure 43-c). Only five females were examined internally: the smallest was immature at $44.6 \mathrm{~cm} L_{\mathrm{T}}$, two females measuring 
$42.7 \mathrm{~cm} L_{\mathrm{T}}$ and $45.9 \mathrm{~cm} L_{\mathrm{T}}$ were mature, and two females $45.2 \mathrm{~cm} L_{\mathrm{T}}$ and $47.8 \mathrm{~cm} L_{\mathrm{T}}$ had egg cases. Each egg bearing female had a single fully developed egg case in each uterus (Figure 43-d).

The egg cases were small (68-70 mm long), relatively broad (anterior case width $30.9-32.9 \%$ of case length), and thick (greatest case height $15.7-20.6 \%$ of case length) with surface smooth to the touch, light brown with a greenish tinge, and with very fine striations. Lateral flanges of case narrow, about $1 \mathrm{~mm}$ wide, flat, and without $\mathrm{T}$-shaped lateral surface, extending length of the egg case. The anterior border of case narrow and concave, with horns narrow, very short, and curved inwards, overlapping slightly, and without any evidence of tendrils being present. The posterior border of case is slightly concave, broad, and with no apparent horns.

This species was encountered from only five sites on the Southwest Indian Ocean Ridge with a depth range of 89 to 1240 m, and may be geographically distinct from its morphometrically similar congener, B. bachi (Figure 42).

Pseudotriakis microdon, de Brito Capello, 1868, False Catshark. A total of 28 P. microdon (10 females and 18 males) were collected with an overall $\mathrm{F}: \mathrm{M}$ sex ratio of 1:1.8, not significantly skewed in favor of males ( $\mathrm{p}$-value<0.05). However, when compared by maturity level, mature males greatly outnumbered females 1:4.5 (pvalue $<0.05$ ), while immature individuals had a sex ratio of 1:1.1 not significantly different from unity (p-value $>0.05$ ).

Males ranged from 135 to $233 \mathrm{~cm} L_{\mathrm{T}}$, with 9 mature (50\% of encountered) (Figure 44-a). Clasper length increased between 200 and $210 \mathrm{~cm} L_{\mathrm{T}}$ (Figure 44-b). The smallest 
mature male measured $213 \mathrm{~cm} L_{\mathrm{T}}$, the largest immature was $198.3 \mathrm{~cm} L_{\mathrm{T}}(91.4 \%$ of $L_{\mathrm{T} \max }$ ), and $L_{\mathrm{T}} 50$ was estimated to be $205.7 \mathrm{~cm} L_{\mathrm{T}}$. Females ranged from 158 to $291.2 \mathrm{~cm}$ $L_{\mathrm{T}}$, with 2 pregnant individuals (Figure 44-c). Oviducal gland width increased between 223 and $226 \mathrm{~cm} L_{\mathrm{T}}$ (Figure 44-d). The smallest mature female measured $267 \mathrm{~cm} L_{\mathrm{T}}$ (91.7\% $L_{\mathrm{T} \max }$ ), the largest immature was $220.8 \mathrm{~cm} L_{\mathrm{T}}$, and $L_{\mathrm{T} 50}$ was estimated to be $243.7 \mathrm{~cm} L \mathrm{~T}$.

Mature females had numerous (estimated several thousand) small oocytes in each ovary. Pregnant females had a single large pup per uterus (Figure 44e-g). A non-term embryo measured $69.1 \mathrm{~cm} L_{\mathrm{T}}$, lacked a bloated stomach full of yolk, and had very large external yolk sac connected by short umbilical cord (Figure 44-f). The largest embryo measured $124.4 \mathrm{~cm} L_{\mathrm{T}}$ and appeared to be of term, and had fully absorbed its yolk sac (Figure 44-g). 


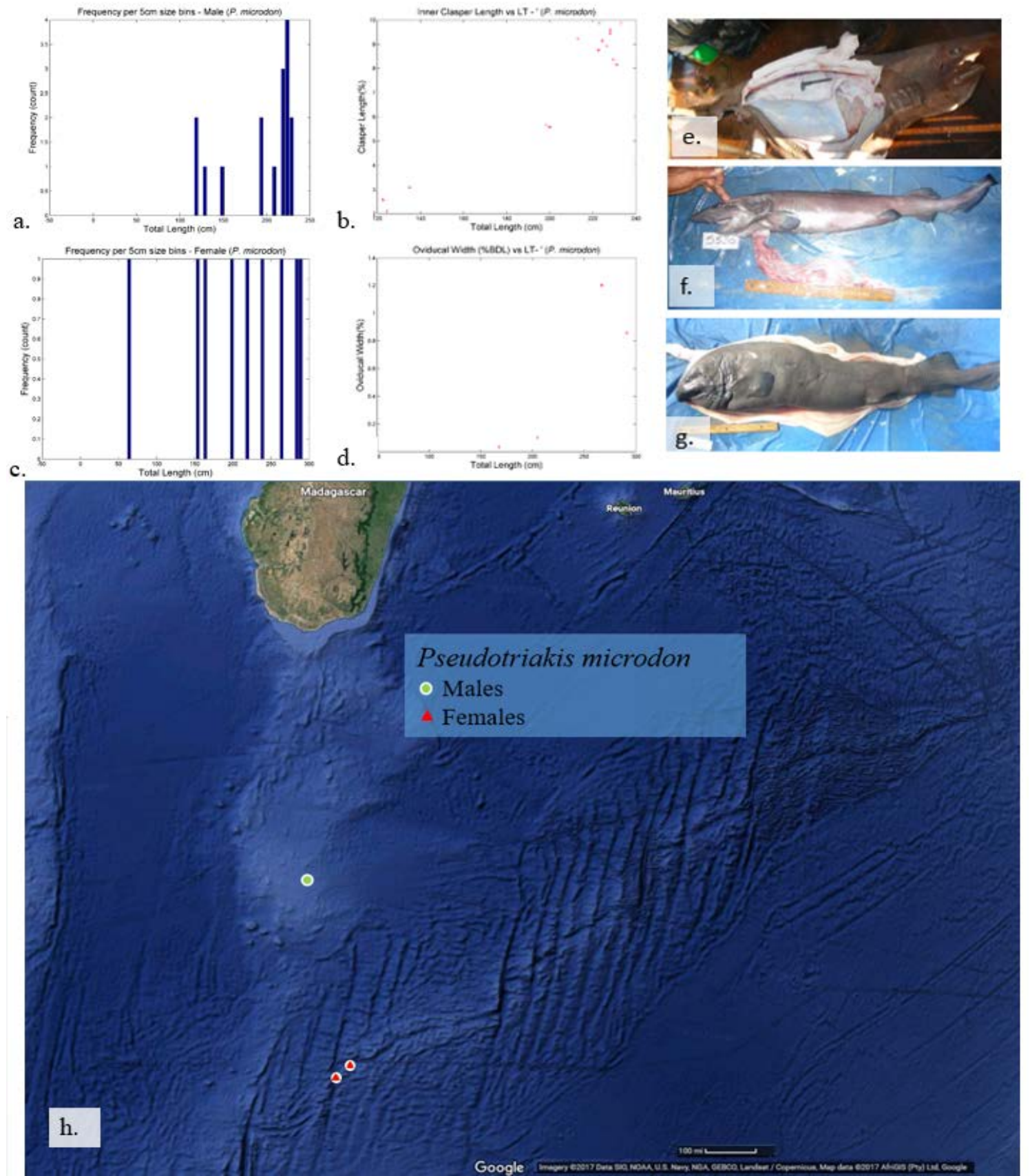

Figure 44. Pseudotriakis microdon: a) size distribution of males, b) relationship between inner clasper length (\%LT) and LT (males), c) size distribution of females, d) relationship between shell gland width (\%LT) and LT (females) ,e) reproductive track of pregnant female with term pup, f) non-term embryo $69.1 \mathrm{~cm} \mathrm{LT}$, g) term embryo $124.4 \mathrm{~cm} \mathrm{LT,} \mathrm{g)}$ distribution of males (green circles), and females (red triangles). Photos by P.J. Clerkin, Map data: Google, Image (c) 2017 DigitalGlobe. 
Stomach contents contained fish (potentially discard from fishing activity) in seven individuals and crustaceans in two specimens. There was no apparent correlation between diet and sex or size.

This species was only encountered from 2 sites in the southwestern part of the Southwest Indian Ocean Ridge between 594 and 1070 m deep, and one site at Walters Shoal between 860 to 1210 meters from (Figure 44-h). Individuals of both sexes were found at every maturity stage at the Southwest Indian Ocean Ridge, while only mature males were present in tows from Walters Shoal.

Chimaera willwatchi, Clerkin, Ebert, and Kemper, 2017, Seafarer's Ghostshark. This was by far the most common species of Chimaera encountered in the region, with a total of 52 C. sp. A were encountered (35 females 17 males) with an overall F:M ratio of 1:0.5, significantly different than unity ( $\mathrm{p}$-value $<0.05$ ). Comparison by maturity stage showed the adult F:M sex ratio was 1:0.7, insignificantly different from unity (pvalue $>0.05$ ), and juvenile F:M sex ratio significantly favored females, 1:0.4 (pvalue $<0.05$ ).

Males ranged from $25.2 \mathrm{~cm} \mathrm{BDL} \mathrm{(47.9} \mathrm{LT)} \mathrm{to} 49.2 \mathrm{~cm}$ BDL (83.4 LT), with 5 mature (29.4\% of encountered) (Figure 45-a). Clasper length increased between $40 \mathrm{~cm}$ BDL and $45 \mathrm{~cm}$ BDL (Figure 45-b) and correlated with a spike in frontal tenaculum length and bulb width. The smallest mature measured $45.6 \mathrm{~cm}$ BDL (92.7\% BDLmax), the largest immature was $49.0 \mathrm{~cm}$ BDL, and $L$ T50 was estimated to be $45.6 \mathrm{~cm}$ BDL. Females ranged from $11.8 \mathrm{~cm}$ BDL $\left(29.0 \mathrm{~cm} L_{\mathrm{T}}\right)$ to $64.5 \mathrm{~cm} \mathrm{BDL}\left(90.0 \mathrm{~cm} L_{\mathrm{T}}\right)$, with 7 mature $(25.6 \%$ total) (Figure 45-c). 

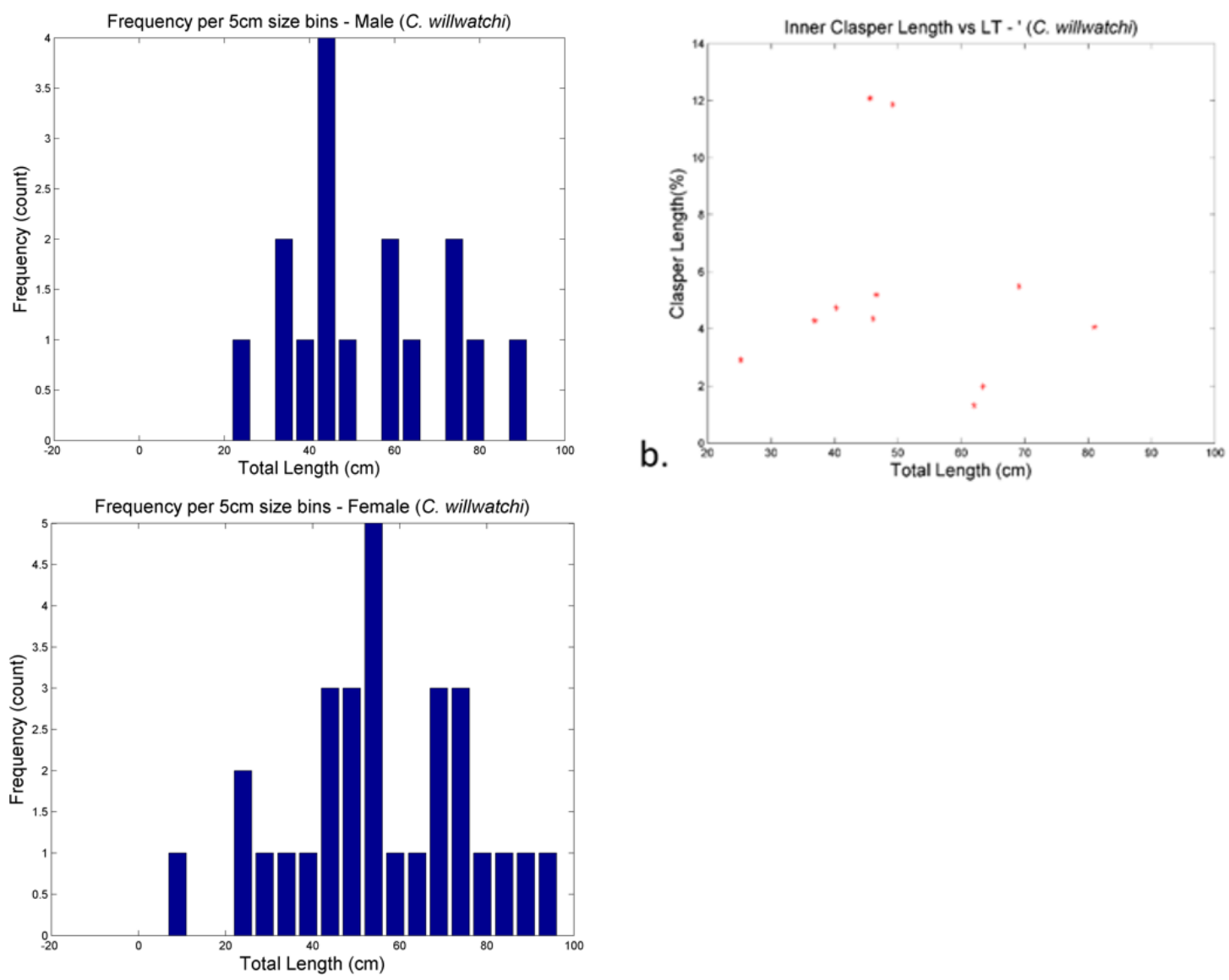

Figure 45. Chimaera willwatchi: a) size distribution of males, b) relationship between inner clasper length (\%LT) and LT (males), c) size distribution of females.

Females matured at a larger size, with the smallest mature female at $51.9 \mathrm{~cm} \mathrm{BDL}$ (76.7 $\mathrm{cm} L_{\mathrm{T}}$ ), and the largest immature was $52.9 \mathrm{~cm} \mathrm{BDL}\left(91.3 \mathrm{~cm} L_{\mathrm{T}}\right.$ ), and $L_{\mathrm{T} 50}$ was estimated to be $51.4 \mathrm{~cm}$ BDL (87\% BDLmax). The smallest free-swimming individual, a female $11.8 \mathrm{~cm} \mathrm{BDL}, 25.8 \mathrm{~cm} L_{\mathrm{T}}$, was white in color with translucent regions on its abdomen suggesting it was recently hatched and of minimum size for the species.

Diet included bony fish (evident by the presence of scales) and bivalves (crushed shells).

Although sex ratios favored females, there appeared to be no intraspecific spatial segregation based solely on sex. However, mature individuals were found exclusively on 
3 sites at the northwestern break of the Southwest Indian Ridge (Figure 46). Mature males were collected at the single farthest northwestern site along the Southwestern Indian Ridge (site 1), and mature females were found on 2 nearby sites to the southeast (sites 2 and 3). Although sites in this region were the only areas where mature individuals were found, mature individuals were in the minority, with all maturity stages present, including a hatchling. Both sexes were found at similar depths ranging between 89 and $1365 \mathrm{~m}$.

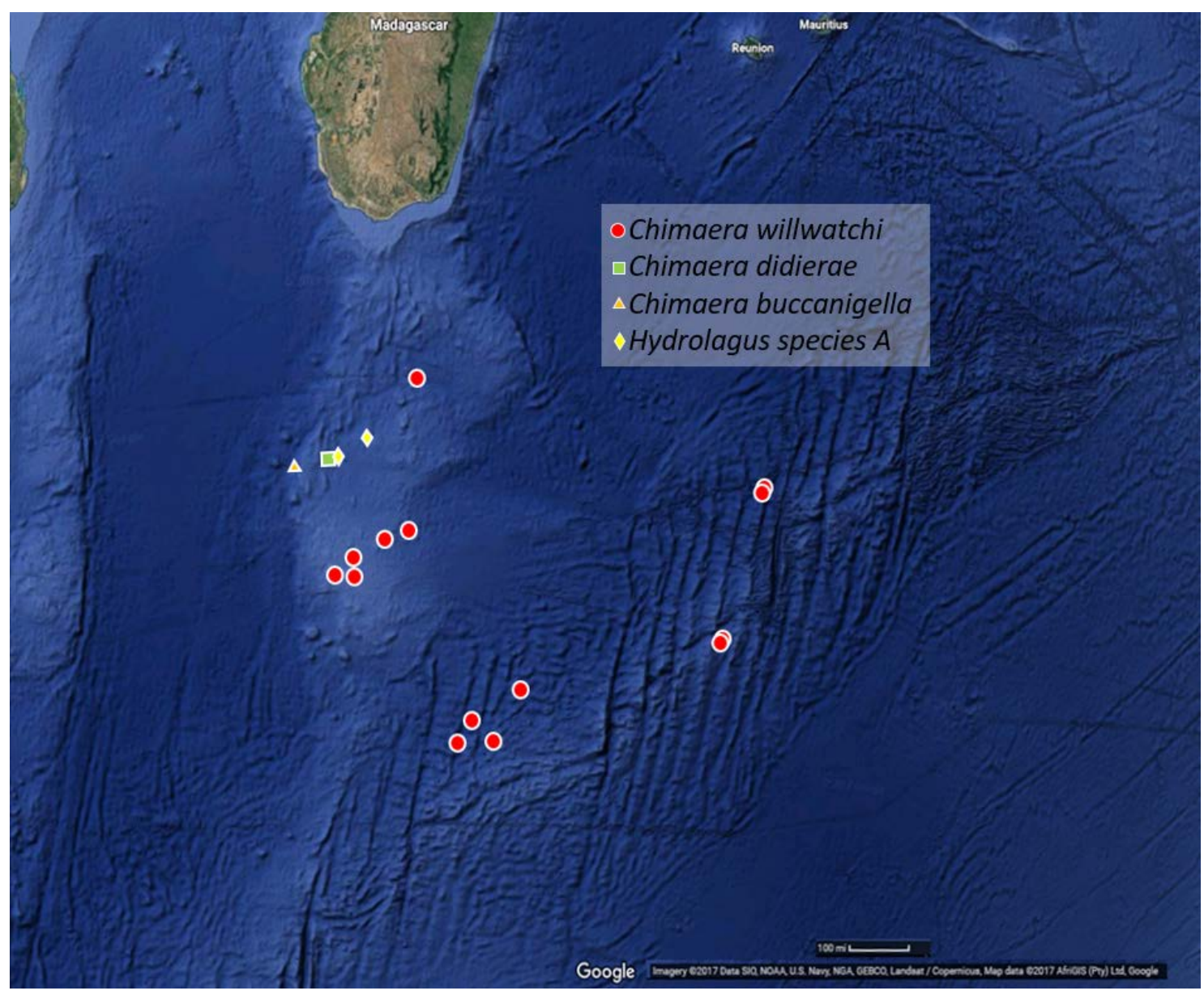

Figure 46. Distribution of Chimaera encountered: Chimaera willwatchi (red circles), Chimaera didierae (green square), Chimaera buccanigella (orange triangle), Hydrolagus species A (yellow diamond). Map data: Google, Image (C) 2017 DigitalGlobe. 
Chimaera didierae, Clerkin, Ebert, and Kemper, 2017, Falkor Chimaera. A single specimen of $C$. didierae, sp. nov. measuring $53.3 \mathrm{~cm} \mathrm{BDL}\left(82.5 \mathrm{~cm} L_{\mathrm{T}}\right)$ was collected from Walters Shoal between 1064 and 1136 m deep (Figure 46). The specimen was female and immature.

Chimaera buccanigella, Clerkin, Kemper, and Ebert, 2017, Dark-mouth Chimaera. A single immature female measuring $40.1 \mathrm{~cm}$ BDL $\left(76.5 \mathrm{~cm} L_{\mathrm{T}}\right)$ was collected from a seamount on Walters Shoal between 495 and 960 m deep (Figure 46).

Hydrolagus sp. A, Imelda's Ghostshark. A mature male and female were collected from a single location on Walters Shoal. A third specimen (immature male) was taken from a nearby seamount, giving an overall F:M ratio of 1:0.5 (p-value >0.05). Males mature between $46.1 \mathrm{~cm}$ BDL $\left(66.0 \mathrm{~cm} L_{\mathrm{T}}\right)$ and $50.0 \mathrm{~cm}$ BDL $\left(690 \mathrm{~mm} L_{\mathrm{T}}\right)$. The females were mature at $55.4 \mathrm{~cm} \mathrm{BDL}\left(73.8 \mathrm{~cm} L_{\mathrm{T}}\right)$. This species was collected between 800 and 1312 m deep (Figure 46).

\section{Discussion}

Sex Ratios. The overall sex ratio for 14 of 26 species where mature male and female individuals were encountered was approximately 1:1 while 10 species were significantly skewed toward females and 2 species were skewed toward males (Table 10). Comparing maturity stages, 12 species had adults with roughly equal sex ratios, and 14 species had significantly skewed ratios. Sex ratios of immature individuals generally were insignificant or followed overall and/or adult ratios, with the exception of Scymnodon plunketi. Overall, and among adults this species was not significantly skewed by sex, but juveniles were significantly skewed toward females (Table 10). Although results in the 
present study did not suggest segregation of S. plunketi based on sex ratios of adults, a separation by size and sex has been reported in the literature (Compagno, 1984).

The frequency of symmetrical and skewed sex ratios was similar, but the significance and direction of skew was closely correlated to reproductive strategy. Viviparous species tended to have strongly skewed adult sex ratios (up to 19:1 in favor of females in Dalatias licha), which has been documented in the literature and theorized to be linked to behavior (Capapé, 2008; Ebert, 2013). High numbers of mature females could indicate sexual segregation after adulthood, potentially as the result of a broader movement pattern or some form of differential habitat use among mature individuals (Grubbs, 2010). This kind of segregation in adults of a species is well documented and considered common in elasmobranchs (Springer, 1967; Yano and Tanaka, 1988; Ebert, 2003). However, since conditions are fairly constant in the deep-sea, reproductive cycles are usually asynchronous, without defined seasonality, and are, therefore, an unlikely influence on segregation in this ecosystem (Wetherbee, 1996; Kyne and Simpfendorfer, 2010). Sexual segregation is likely influenced by environmental factors such as diet, and differential foraging patterns that could be a function of different caloric requirements associated with each sex’s role in reproduction (Grubbs, 2010). Compared to oviparous females, live-bearing females have a larger energetic investment in their young and likely require higher calorie food items than their male counterparts. Notably, this strong sexual segregation is not apparent in the oviparous catshark species in this study and has been observed to be absent in other egg laying species (Bullis 1967; Ebert, 2005; Bizzarro et al., 2014). 
Maximum Length. Of the 16 species with previously established maximum lengths, nine met or exceeded the reported maximum total lengths; these included C. granulosus, D. profundorum, E. granulosus, E. lucifer, E. sculptus, C. coelolepis, C. crepidater, Z. squamulosus, and A. sinensis (Table 9). Four other species, D. calcea, E. pusillus, C. owstonii, and P. microdon, were within $90 \%$ of the reported maximum $L_{\mathrm{T}}$ at $6 \mathrm{~cm}$ (95.1\%), $4.5 \mathrm{~cm}$ (91.0\%), $7 \mathrm{~cm}$ (94.2\%), and $4.8 \mathrm{~cm}$ (98.7\%), respectively (Table 9). The remaining three species were well within their known ranges: C. squamosus (82.3\%), D. calcea (78.3\%), and S. plunketi (81.9\%) (Table 9).

Table 9c. A list of species encountered and lengths reported in the literature for Centrophoridae and Etmopteridae.

\begin{tabular}{|c|c|c|c|}
\hline Species & Sex & Reported Lengths $(\mathrm{cm})^{\star}$ & Reference \\
\hline \multicolumn{4}{|l|}{ Centrophoridae } \\
\hline \multirow[t]{3}{*}{ Centrophorus granulosus } & Male LT of Maturity & $110-128$ & Compagno, 1984; Ebert, 2013; White et al., 2013 \\
\hline & Female LT of Maturity & $>130$ & \\
\hline & $\operatorname{Max} * *$ & 170 & \\
\hline \multirow[t]{3}{*}{ Centrophorus squamosus } & Male LT of Maturity & 100 & Compagno, 1984; Compagno and Niem 1998; Girard and Du Buit 1999, Clarke et al. 2001; Ebert, 2013 \\
\hline & Female LT of Maturity & $105-125$ & \\
\hline & $\operatorname{Max}$ & 164 & \\
\hline \multirow[t]{3}{*}{ Deania calcea } & Male LT of Maturity & 80 & Compagno, 1984; Cox and Francis, 1997; Clarke et al. 2002; Ebert, 2013 \\
\hline & Female LT of Maturity & $90-100$ & \\
\hline & $\operatorname{Max}$ & 122 & \\
\hline \multirow[t]{3}{*}{ Deania profundorum } & Male LT of Maturity & $43-67$ & Compagno, 1984; Bass et al., 1986; Ebert, 2013 \\
\hline & Female LT of Maturity & $60-77$ & \\
\hline & $\operatorname{Max}$ & $79.0-104$ & \\
\hline \multicolumn{4}{|l|}{ Etmopteridae } \\
\hline \multirow[t]{3}{*}{ Etmopterus alphus } & Male LT of Maturity & 32.5 & Ebert et al., 2016 \\
\hline & Female LT of Maturity & 33 & \\
\hline & $\operatorname{Max}$ & $33.6(\mathrm{M}) 39$ (F) & \\
\hline \multirow[t]{3}{*}{ Etmopterus bigelowi } & Male LT of Maturity & $40-67$ & Bernardes et al, 2005; Ebert, 2013 \\
\hline & Female LT of Maturity & $50-65$ & \\
\hline & $\operatorname{Max}$ & 67 & \\
\hline \multirow[t]{3}{*}{ Etmopterus compagnoi } & Male LT of Maturity & $48-68$ & Ebert, 2013 \\
\hline & Female LT of Maturity & $53-63$ & \\
\hline & $\operatorname{Max}$ & $78.0-79$ & \\
\hline \multirow[t]{3}{*}{ Etmopterus granulosus } & Male LT of Maturity & $46-68$ & Compagno, et al, 1989; Lamilla 2003; Ebert 2013 \\
\hline & Female LT of Maturity & $62-86$ & \\
\hline & $\operatorname{Max}$ & 86 (F); 88 (Est.) & \\
\hline \multirow[t]{3}{*}{ Etmopterus pusillus } & Male LT of Maturity & $31-39$ & Compagno, et al, 1989; Bianchi et al, 1999; Shirai and Tachikawa 1993; Coelho and Erzini 2005 \\
\hline & Female LT of Maturity & $38-47$ & \\
\hline & Max & $47.9(\mathrm{M}), 50.5$ (F) 100 (Est.) & \\
\hline \multirow[t]{3}{*}{ Etmopterus sculptus } & Male LT of Maturity & $43.5-44.2$ & Ebert et al., 2011 \\
\hline & Female LT of Maturity & $47.4-50.1$ & \\
\hline & $\operatorname{Max}$ & $44.2(\mathrm{M}), 50.1(\mathrm{~F})$ & \\
\hline
\end{tabular}


Table 9d. A list of species encountered and lengths reported in the literature for Somniosidae, Dalatiidae, scyliorhinids and Pseudotriakidae.

\begin{tabular}{|c|c|c|c|}
\hline Species & Sex & Reported Lengths $(\mathbf{c m})^{\star}$ & Reference \\
\hline \multicolumn{4}{|l|}{ Sominosidae } \\
\hline \multirow[t]{3}{*}{ Scymnodon plunketi } & Male LT of Maturity & $100-120$ & Compagno, 1984; Ebert, 2013; White et al., 2013 \\
\hline & Female LT of Maturity & $130-145$ & \\
\hline & $\operatorname{Max}$ & 170 & \\
\hline \multirow[t]{3}{*}{ Centroscymnus coelolepis } & Male LT of Maturity & $70-86$ & Yano and Tanaka 1988; Last and Stevens 1994; Girard and Du Buit 1999 \\
\hline & Female LT of Maturity & $100(\mathrm{~F})$ & \\
\hline & $\operatorname{Max}$ & $120-158$ & \\
\hline \multirow[t]{3}{*}{ Centroscymnus owstonii } & Male LT of Maturity & $70-79$ & Cox and Francis, 1997; Yano and Tanaka $(1987,1988)$ and Daley et al. (2002), \\
\hline & Female LT of Maturity & $82-105$ & \\
\hline & $\operatorname{Max}$ & 121 & \\
\hline \multirow{3}{*}{ Centroselachus crepidater } & Male LT of Maturity & $60-68$ & Bass et al., 1986; Daley et al., 2002; Ebert, 2013 \\
\hline & Female LT of Maturity & $77-88$ & \\
\hline & Max & $105-130$ & \\
\hline \multirow[t]{3}{*}{ Zameus squamulosus } & Male LT of Maturity & $47-51$ & Last and Stevens 1994; Ebert, 2013 \\
\hline & Female LT of Maturity & $59-69$ & \\
\hline & $\operatorname{Max}$ & 84 & \\
\hline \multicolumn{4}{|l|}{ Dalatiidae } \\
\hline \multirow[t]{3}{*}{ Dalatias licha } & Male LT of Maturity & $77-121$ & Compagno, 1984; Bauchot 1987; Springer, 1991; Ebert, 2013 \\
\hline & Female LT of Maturity & $117-159$ & \\
\hline & Max & 182 & \\
\hline \multicolumn{4}{|l|}{ Scyliorhinid catsharks } \\
\hline \multirow[t]{3}{*}{ Apristurus sinensis } & Male LT of Maturity & 47 & Last and Stevens 2009 \\
\hline & Female LT of Maturity & $61(\mathrm{~F})$ & \\
\hline & Max & 75: 82(Est.) & \\
\hline \multicolumn{4}{|l|}{ Pseudotriakidae } \\
\hline \multirow[t]{3}{*}{ Pseudotriakis microdon } & Male LT of Maturity & $261-295$ & Compagno, 1984; Yano, 1992; Ebert, 2013 \\
\hline & Female LT of Maturity & $\sim 275$ & \\
\hline & Max & $269(\mathrm{M}), 296(\mathrm{~F})$ & \\
\hline \multicolumn{4}{|c|}{${ }^{*} \mathrm{~F}=$ females, $\mathrm{M}=$ males, Est. $=$ Estimated } \\
\hline \multicolumn{2}{|c|}{${ }^{* *}$ Unsexed unless indicated otherwise } & & \\
\hline
\end{tabular}

Sexual Dimorphism. Many species encountered during this survey exhibited sexual dimorphism in the form of length. Observations suggest size differences between sexes might be linked to reproductive mode (Table 9). The females of viviparous species in this study consistently exhibited greater $L_{\mathrm{T}}$ than their male counterparts. In some viviparous species, females encountered were 1.4 (D. licha), 1.5 (C. crepidater), and 1.8 (Z. squamulosus) times the length of the largest mature males.

The maximum length for 13 viviparous species studied and $L_{\mathrm{T} 50}$ of all viviparous species calculated (seven species) showed females ultimately obtained larger size $\left(L_{\mathrm{Tmax}}\right)$ and matured at a greater length than males, (Table 9). The remaining five viviparous species did not have confirmed mature representatives of both sexes. 
In contrast, the oviparous scyliorhinids species of this study (all single egg case per uterus) did not exhibit clear sexual dimorphism in the form of length, showing similar sizes between the sexes with only Apristurus cf. melanoasper having drastically larger males $(77.0 \mathrm{~cm}$ vs $47.7 \mathrm{~cm})$. However, the sample size for this species was small (3 males, 2 females) with no female maturity status confirmed by internal evaluation. Previous studies of scyliorhinids have also observed males being equal or even larger than females (Compagno, 1984; Cross, 1988; Richardson et al., 2000; Musick and Ellis, 2005; Ebert et al., 2006).

It is worth noting that although they are single egg oviparous, Chimaera willwatchi (the only chimaeroid with mature males and females encountered during these surveys) had females 1.3 times the largest mature male (Table 9). The family shows sexual dimorphism in the form of frontal tenaculum, and paired prepelvic tenacula (present in males) in addition to pelvic claspers and, in some species, shape of pelvic fins, and body color (Didier, 1998; Didier et al., 2012).

Chimaeroids lay two egg capsules simultaneously, and while the annual number of spawn is unknown, Hydrolagus colliei has been observed to lay a pair of eggs every seven to 10 days (Didier et al., 1998). Females are believed to store sperm (Smith et al., 2001) and this rate is assumed for other species over a spawning season of several months (Didier et al., 2012).

Pseudotriakis microdon, the only oophagous species encountered, exhibited females also 1.3 times the largest male. Females carry a single pup per uterus, which cannibalizes eggs (Yano, 1992) until they reach up to $42.7 \%$ of their mother's body length (Table 9). 
Though low in number this litter takes up a considerable volume, which might lead to the same anatomical challenges of large litter bearing viviparous species.

Maturity. Species in this study matured within a well-defined, fairly narrow size range, with an extended juvenile stage followed by either rapid maturation or a maturation stage of little growth. This observation has been documented in previous studies (Holden, 1974; Walker \& Hislop, 1998). Most species studied here matured in excess of $80 \%$ of their maximum length; the exceptions being E. granulosus ( $55.9 \% L_{\mathrm{T}}$ males, $58.9 \% L_{\mathrm{T}}$ females) and C. crepidater $\left(66.6 \% L_{\mathrm{T}}\right.$ males and $33.6 \% L_{\mathrm{T}}$ females). This agrees with Holden (1974), who observed that elasmobranchs usually matured between $60 \%$ and $90 \%$ of their maximum length. Since maturity as a percent of maximum length has been linked to reproductive output and abundance (Holden, 1974), it is interesting that the two outliers, E. granulosus and C. crepidater, were by far the most common shark species encountered, making up more than half of all the individuals in this study (Table 9). It is possible that earlier maturation could provide the populations with more resilience and a relatively large capacity to respond to a perturbation.

Size at maturity was greater for females than males for all viviparous species with maturity confirmed by either internal examination or observed pupping (C. granulosus, D. calcea, D. profundorum, E. granulosus, E. lucifer, E. sculptus, S. plunketi, C. coelolepis, C. owstonii, C. crepidater, and D. licha). Greater length of live bearing females at maturation is consistent with the literature (Cortes, 2000). This relationship was observed across various forms of viviparity including the only oophagous species surveyed (Pseudotriakis microdon), which also had females showing greater length of 
maturity relative to males, illustrating, perhaps, the same anatomical size demand imposed on females of other live bearing reproductive strategies. In order to preserve specimen integrity, due to limited specimens and outstanding taxonomic resolution for some species, most oviparous species, e.g. catsharks and chimaeras, were not examined internally. However, based on the few females found to have egg cases, oviparous females appear to mature at the same size as their male counterparts. It is feasible that as a reproductive strategy, oviparity does not have the same anatomical size demand as viviparity.

Estimates of $L_{\mathrm{T} 50}$ were between length of smallest mature and largest immature with the exception of male Centroselachus crepidater and Apristurus cf. manis. In both cases the estimate was low $(2.8 \mathrm{~cm}$ and $3.6 \mathrm{~cm}$, respectively). Since $L \mathrm{~T} 50$ is a theoretical estimate of a length (where $50 \%$ of the individuals of this length will be mature), small sample size, outliers, or limited maturity data can skew this binomial estimation (Ebert, 2005). In contrast, first maturity is an observed and measured parameter of maturity. While $L_{T 50}$ provides a better maturity estimate of a population as a whole, first maturity is helpful with limited sample sizes or sample bias (resulting from behavior or segregation), and outliers (Ebert, 2005).

Mature Oocyte Number. Of the eight species found to have mature uterine oocytes (eggs) (Table 11), only Etmopterus granulosus had a bilateral distribution bias, with significantly disproportionate number of eggs favoring the left uterus (7.1 left vs 6.5 right), while the other seven species did not have statistically significant egg distribution bias between left and right uteri. The latter condition is most common among 
chondrichthyans (Holden, 1975; Braccini and Chiaramonte, 2002; Mabragaña et al., 2002; Ebert, 2005). On average, there was a higher number of ovarian oocytes than uterine eggs or pups, suggesting larger litters than indicated by fetal counts alone as inferred by Ebert (2013). Specimens with mature uterine eggs had dormant ovaries (inactive at time of inspection as indicated by the absences of healthy, developing oocytes), suggesting a resting phase.

A higher number of E.granulosus ( $\mathrm{n}=595)$ were encountered with mature eggs than any other species. Females had 14 mature eggs on average with up to 38 oocytes per ovary, agreeing with Wetherbee’s (1996) observations of nine to 15 eggs (per uterus), with up to 39 between both uteri, and seven to 30 ovarian eggs, in specimens off New Zealand.

There was no correlation between size of mothers and number of eggs in any of the species studied. This contrasts with observations made by Peres and Vooren (1991) on viviparous School Sharks (Galeorhinus galeus), which had a direct relationship between length and fecundity, but agrees with Holden's (1975) examination of Raja clavata (an oviparous species) maternal length and fecundity, in which he concluded body size not to be a limiting factor. Since the ovarian and uterine activity of this study appeared to alternate, it is not possible to directly link ovarian productivity to uterine yield or overall fecundity. Because deep-sea shark species are poorly understood, there is very little information comparing female length and number of oocytes or reproductive output.

Fecundity. Seven species (E. alphus, E. granulosus, S. plunketi C. coelolepis, C. crepidater, Z. squamulosus, and P. microdon) were encountered with litters at different 
stages of development indicating no defined breeding season (Wetherbee, 1996; Kyne and Simpfendorfer, 2010) (Table 12). All litters examined fell within known ranges. Etmopterus granulosus had two to 15 pups comparable to the nine to 16 off Australia and six to 15 off New Zealand reported by Wetherbee (1996) and Ebert (2013). The single pregnant Scymnodon plunketi encountered had 20 pups while the species is known to have up to 30 young (Garrick, 1959a; Compagno, 1984; Ebert, 2013). The only pregnant Centroscymnus coelolepis had 12 pups compared to one to 29 (mostly between 12 and 14) (Garrick, 1959b; Cox and Francis, 1997; Ebert, 2013). Centroselachus crepidater had two to seven pups compared to four to six reported by Last and Stevens (1994) and one to nine (with an average of six) from Ebert, 2013.

This is the first record of pregnant females for two species, E. alphus (not examined internally) and Z. squamulosus, and first the account of fecundity for the latter. A single pregnant Z. squamulosus was encountered and found to have three pups (all present in the left uterus), thereby confirming previous estimates of litter sizes from three to 10 pups (Ebert, 2013). Because spontaneous abortion upon capture was fairly common, fecundity in terms of number of offspring is probably in the upper end of the ranges presented in this study. The species is viviparous with external yolk sac dependency as presumed by White et al (2006).

Two pregnant Pseudotriakis microdon were encountered during this study. These females had no more than a single pup per uterus, which supports the observation by Yano (1992) of in utero cannibalism in this species. Yano (1992) found the stomachs of embryonic Pseudotriakis microdon to contain yolk and egg capsules, implying oophagy, 
with pups consuming yolk to replenish their external yolk sac reserves during the final stages of gestation. The pre-term embryo of this study measured $69.1 \mathrm{~cm} L_{\mathrm{T}}$ with considerable yolk reserves (Figure 44-f), while term embryos measured 122.9 and 124.4 cm exceeding those investigated by Yano (1992) which measured $44.7-120.2 \mathrm{~cm}$. Term embryos of this study had no yolk reverses and appeared ready for birth (Figure 44-g). The larger pup was active and able to swim free once removed from the uterus. Pups of this study likely represent an accurate minimum free-swimming length for the species.

Egg Cases. Egg cases are a useful diagnostic tool in identifying shark species (Hubbs and Ishiyama, 1968; Ebert, 2005). Of the 12 oviparous species examined, only two (B. bachi and B. naylori) were found to have egg cases in utero (Figs. 41-b and 43-d respectively). The genus Bythaelurus has two different reproductive modes: 1) single egg oviparity where a single tough, leathery egg case is carried per uterus and deposited on the seabed during an early stage of development, and 2) viviparity where embryos are carried inside flimsy, membranous egg cases and hatch within the uteri before live birth (Francis, 2006b; Carrier et al., 2004; Ebert et al., 2006; Ebert and Clerkin, 2015). Based on the observation of a single, rigid and leathery egg case per uterus, the reproductive mode of both Bythaelurus species in this study was determined to be single egg oviparity. Egg cases are unique to each species and the descriptions have been included to serve as useful tool in for identifying specimens and potential nursery grounds.

Neonates. Free-swimming neonates of six species (Etmopterus granulosus, Centroselachus crepidater, Somniosus cf rostratus, Dalatias licha, Pseudotriakis microdon, and Chimaera willwatchi) were identified by presences of umbilical scars in 
live bearing species and translucent, developing abdomens in the Chimaeroids. Neonates were encountered rarely, but their presence along with pregnant females primarily along the Southwest Indian Ocean Ridge indicates a potential pupping ground and nursery.

Diet. During the study, 194 individuals from 12 species were found to have food items in their stomachs (Table 13, Fig 26). Diet composition for these sharks was chiefly teleost, with boney fish representing $70.9 \%$ of stomach contents. Squid was the primary food item in $22.8 \%$ of diets, with crustaceans (3.2\%), mammals - all pinniped - (1.9\%), and sharks (1.3\%) being the primary food sources in a much smaller percentage of individuals.

Prey items usually fell within the known food items for all species with the exception of crustacean and mammal remains in C. crepidater. Centrophorus granulosus of this study were found exclusively with bony fishes in their stomachs, which agrees with Compagno, 1984. One specimen of C. squamosus had stomach contents that included cephalopod remains in agreement with its presumed diet of fish and cephalopods (Macpherson, 1989; Last and Stevens, 1994; Ebert, 2013). Deania calcea of this study ate bony fish and cephalopods, the species’ known diet (Compagno et al, 1989). The stomach contents of adult D. profundorum had bony fish and squid, consistent with Ebert et al. 1992. Etmopterus granulosus consumed a wide variety of bony fishes and cephalopod as stated by Compagno et al, 1989. Scymnodon plunketi diet was comprised largely of bony fishes, with one individual feeding on cephalopods, agreeing with Ebert (2013). Centroscymnus coelolepis stomach contents included bony fish, cephalopod, and mammal remains, consistent with Last and Stevens (1994) and Compagno et al. (1989). 
Centroscymnus owstonii diet was composed of cephalopods, bony fishes in agreement with Last and Stevens (1994). Centroselachus crepidater, diet contained fishes, cephalopod, crustacean, and, in one specimen, mammalian (pinniped) remains. While fish and cephalopod have been recorded in the literature (Macpherson, 1989; Last and Stevens 1994), this is the first account of crustacean and mammal in this species' diet. Dalatias licha diet was mainly bony fishes, with some shark, squid, and other invertebrates as described by Wetherbee et al. in 1990. Pseudotriakis microdon stomach contents contained fish and crustaceans in two specimens, which matched a study by Yano and Musick (1992). Chimaera willwatchi diet included bony fish and bivalves (evident by the presence of scales and crushed shells, respectively). This is the first diet investigation for Chimaera willwatchi, but benthic invertebrates have been the predominate prey of all chimaeroid studied to date, with a few species, Chimaera monstrosa Linnaeus, 1758, Hydrolagus bemisi Didier, 2002, Hydrolagus colliei (Lay and Bennett, 1839), Hydrolagus novaezealandiae (Fowler, 1910), Harriotta raleighana Goode \& Bean, 1895, known to consume small fish (Didier et al., 2012; Dunn et al., 2010). There was no trend between diet composition and sex, maturity, or length of individual for any species, and it appears diet might be based on opportunity (Grubbs, 2010).

This study divided diet items into broad caloric groups and presented these results in each species account. However, based on the food items found, the sharks of this study feed at relatively high trophic levels supporting the view of sharks as top marine predators (Cortes, 1999). Sharks are commonly assumed to be top-level consumers in 
marine food webs, yet studies calculating trophic positions are very few (Cortes, 1999). A more in depth study and analysis is needed to determine each species position in the trophic web.

Distribution. The Southwestern Indian Ocean Offshore is divided into two main geologically distinct ecosystems: the Southwest Indian Ocean Ridge and the Madagascar Ridge (which includes the relatively shallow Walters Shoal) (Sinha et al,. 1981). Surveyed species were distributed unevenly between these ecosystems (Tables 14 and 15). Although its benthic habitat is less complex (Goslin et al., 1980; Sinha et al., 1981; Collette and Parin, 1991), the Walters Shoal region of the Madagascar Ridge had a far greater amount of biodiversity, yielding a total of 25 species (80.6\% of all encountered), with 11 species (35.5\% of all encountered) found nowhere else during this survey (Table 14). The Southwest Indian Ocean Ridge had fewer species overall, with 17 species encountered (54.8\% of total), and seven species (6.5\% of total) unique to this ecosystem during this study (Table 14). 
Table 14. Distribution of species encountered during surveys and their reported ranges

\begin{tabular}{|c|c|c|c|c|}
\hline Species & Ecosystem ${ }^{* \star}$ & Region & Reported Ranges* & Reference \\
\hline \multicolumn{5}{|l|}{ Centrophoridae } \\
\hline Centrophorus granulosus & WS, SWIOR & SW Ind & Att, W Atl, E Att, W Ind, E Ind, SW Pac, Pac & White et al, 2013 \\
\hline Centrophorus squamosus & WS, SWIOR & SW Ind & E Att, W Ind, W Pac & Compagno et al., 1989; Acuñe-Marrero et al., 2013 \\
\hline Deania calcea & WS, SWIOR & SW Ind & E Att, W Pac, E Pac, Ind & Compagno, 1984; Compagno and Niem, 1998; Long, 1997, Ebert, 2013 \\
\hline Deania profundorum & wS & SW Ind & NW At, E At, W Central Pac, Indo-W Pac, Arabian S & Compagno et al., 1989; Jawad et a1, 2013 \\
\hline \multicolumn{5}{|c|}{ 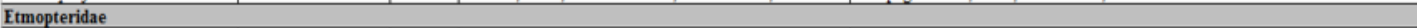 } \\
\hline Etmopternis alphus & WS, SWIOR & SW Ind & SW Ind & Ebert et al., 2016 \\
\hline Etmopterus bigelowi & ws & SW Ind & Att, W Atl. Ind, W Pac & Bernardes et al, 2005 \\
\hline Etmopterus compagnot & NMR & SW Ind & SE Atl, Ind & Bass et al., 1986; Ebert, 2013 \\
\hline Etmopterus granulosus & WS, SWIOR & SW Ind & SE Pac, SW Pac, SW Att, SE Att, Ind & Compango, 1984; Ebert, 2013 \\
\hline Etmoptenus pusillius & WS & SW Ind & W Atl, E Adt, W Ind, W Pac, SE Pac & Compagno, 1984; Last, and Stevens, 1994; Cox and Francis, 1997 \\
\hline Etmopterus scuiptus & Ws & SW Ind & Att, Ind & Ebert et al., 2011; Ebert, 2013 \\
\hline Etmopterus ef sculptus & WS, SWIOR & SW Ind & First account & This publication \\
\hline \multicolumn{5}{|l|}{ Sominosidae } \\
\hline Scymmodon phunkett & WS, SWIOR & SW Ind & SW Pac, Ind & Ebert, 2013 \\
\hline Centroscymnus coelolepis & WS, SWIOR & SW Ind & W Att, E Atl, W Med, W Pac, W Central Pac, Ind & Claro, 1994; Last and Stevens, 1994; Compagno, 1998; Ebert, 2013 \\
\hline Centroscymmus owstonili & WS, SWIOR & SW Ind & W Central Att, W Pac, SE Pac, Ind & Compagno, 1984; Ebert, 2013 \\
\hline Centroselachus crepidater & WS, SWIOR & SW Ind & E Atl, Ind, W Pac, SE Pac & Compagno, 1984; Ebert, 2013 \\
\hline Zameus squamulosus & WS & SW Ind & W Ind, E Pac, W Pac, E Att, W Atl & Compagno et al., 1989; Mundy, 2005; Ebert, 2013; Ebert et al., 2014 \\
\hline Somniosus of rostratus & SWIO & SW Ind & First account & This publication \\
\hline \multicolumn{5}{|l|}{ Dalatiidae } \\
\hline Dalatias licha & WS, SWIOR & SW Ind & W Atl, E Atl, W Me, W Ind, W Pac, Central Pac & Compango, 1984; Ebert, 2013 \\
\hline \multicolumn{5}{|l|}{ Scyliorhinid catsharks } \\
\hline Apristurus sinersis & WS, SWIOR & SW Ind & NW Pac, Ind, Presence in the W Central Pac is uncert & Carpenter and Niem, 1998; Ebert, 2013 \\
\hline Apristurus sp. cf. albisoma & SWIOR, NMR & SW Ind & First account & This publication \\
\hline Apristurus sp. cf. ampliceps 1 & WS, SWIOR & SW Ind & First account & This publication \\
\hline Apristurus sp. ef. ampliceps 2 & WS, SWIOR & SW Ind & First account & This publication \\
\hline Apristurus sp. cf. manis & WS, SWIOR & SW Ind & First account & This publication \\
\hline Apristurus sp. cf. melanoasper & WS & SW Ind & First account & This publication \\
\hline Bythaehurus bachi & Ws & SW Ind & SW Ind & Weigmann et al., 2016 \\
\hline Bythaeiurus nayiori & SWIO & SW Ind & SW Ind & Ebert and Clerkin, 2015 \\
\hline \multicolumn{5}{|l|}{ Pseudotriakidae } \\
\hline Pseudotriakis microdion & WS, SWIOR & SW Ind & W Att, SE Att, NE Att, Ind, Pac & Compagno, 1998; Claro, 1994; Soto, 2001; Ebert, 2013; Clerkin and Ebert, 2017 \\
\hline \multicolumn{5}{|l|}{ Chimaeridae } \\
\hline Chimaera willwatcht & NMR, WS, SWIOR & SW Ind & SW Ind (first account) & Clerkin et al, in press \\
\hline Chimaera didierae & WS & SW Ind & SW Ind (first account) & Clerkin et al, in press \\
\hline Chimaera buccanigella & WS & SW Ind & SW Ind (first account) & Clerkin et al, in press \\
\hline Hydrolagus imeldae & WS & SW Ind & SW Ind (first account) & Clerkin et al, in press \\
\hline *Abreviations: Atl- Atlantic, Ind- & -Indian, Med-Mediter & franean, $\mathrm{Pa}$ & ac-Pacific & \\
\hline $\mathrm{N}=$ North, $\mathrm{S}=$ South, $\mathrm{W}=$ West(e & rn), E=East(ern) & & & \\
\hline **Abreviations: NMR-Northern & Madagascar Ridge, & & is Shoal, SWIOR- Southwe & \\
\hline
\end{tabular}

Table 15. SIMPER pairwise comparison of species composition between regions. The contributing and cumulative Bray Curtis similarity (species contributions) percentages are expressed as \%.

Walters Shoal average similarity: 34.60

\begin{tabular}{|l|r|r|r|r|r|}
\hline Species & Av.Abund & Av.Sim & Sim/SD & Contrib\% & Cum.\% \\
\hline \hline E. granulosus & 3.15 & 22.08 & 1.6 & 63.82 & 63.82 \\
\hline C. crepidater & 1.03 & 4.22 & 0.75 & 12.2 & 76.02 \\
\hline D. licha & 0.63 & 2.45 & 0.37 & 7.07 & 83.09 \\
\hline A. sinensis & 0.59 & 1.87 & 0.54 & 5.4 & 88.49 \\
\hline C. coelolepis & 0.27 & 0.74 & 0.38 & 2.14 & 90.63 \\
\hline
\end{tabular}

Southwest Indian Ocean average similarity: 37.33

\begin{tabular}{|l|l|l|l|l|l|}
\hline Species & Av.Abund & Av.Sim & Sim/SD & Contrib\% & Cum.\% \\
\hline \hline
\end{tabular}




\begin{tabular}{|l|r|r|r|r|r|} 
E. granulosus & 3.96 & 27.55 & 1.25 & 73.78 & 73.78 \\
\hline C. crepidater & 1.02 & 5.4 & 0.72 & 14.46 & 88.24 \\
\hline D. licha & 0.29 & 1.47 & 0.31 & 3.95 & 92.19 \\
\hline
\end{tabular}

Bottom and mid-water trawls had considerably different species composition with the majority of sharks encountered caught in bottom trawls (Table 16). The three most abundant species were found in high numbers in both gear types, with E. granulosus and C. crepidater more common in bottom trawls and D. licha most abundant in mid-water trawls. The other species were more much common in bottom trawls with the exception of $C$. squamosus which was similar in abundance in each habitat (Table 16 and Figure 47).

Table 16. SIMPER pairwise comparison of species composition between trawl gear types. The contributing and cumulative Bray Curtis similarity (species contributions) percentages are expressed as \%.

Group Bottom Trawl average similarity: 42.69

\begin{tabular}{|l|r|r|r|r|r|}
\hline Species & Av.Abund & Av.Sim & Sim/SD & Contrib\% & Cum.\% \\
\hline E. granulosus & 4.54 & 30.45 & 1.79 & 71.32 & 71.32 \\
\hline C. crepidater & 1.3 & 6.12 & 0.92 & 14.32 & 85.65 \\
\hline A. sinensis & 0.55 & 1.45 & 0.45 & 3.39 & 89.04 \\
\hline S. plunketi & 0.45 & 0.82 & 0.29 & 1.91 & 90.95 \\
\hline
\end{tabular}

Group Midwater Trawl average similarity: 32.34

\begin{tabular}{|l|r|r|r|r|r|}
\hline Species & Av.Abund & Av.Sim & Sim/SD & Contrib\% & Cum.\% \\
\hline E. granulosus & 1.69 & 20.2 & 1 & 62.46 & 62.46 \\
\hline D. licha & 0.43 & 5.72 & 0.52 & 17.69 & 80.15 \\
\hline C. crepidater & 0.41 & 4.08 & 0.53 & 12.61 & 92.77 \\
\hline
\end{tabular}

Groups Bottom Trawl and Midwater Trawl average dissimilarity = 69.85 
Bottom Midwater

Trawl Trawl

Species Av.Abund Av.Abund Av.Diss Diss/SD Contrib\% Cum.\%

\begin{tabular}{|c|c|c|c|c|c|c|}
\hline E. granulosus & 4.54 & 1.69 & 25.98 & 1.51 & 37.19 & 37.19 \\
\hline C. crepidater & 1.3 & 0.41 & 8.32 & 1.12 & 11.9 & 49.1 \\
\hline D. licha & 0.39 & 0.43 & 5.04 & 0.82 & 7.21 & 56.31 \\
\hline A. sinensis & 0.55 & 0.07 & 3.69 & 0.92 & 5.28 & 61.59 \\
\hline S. plunketi & 0.45 & 0.1 & 3.47 & 0.65 & 4.97 & 66.56 \\
\hline C. willwatchi & 0.29 & 0.13 & 2.71 & 0.69 & 3.88 & 70.44 \\
\hline C. coelolepis & 0.33 & 0.1 & 2.61 & 0.76 & 3.73 & 74.17 \\
\hline C. granulosus & 0.23 & 0.08 & 2.24 & 0.5 & 3.21 & 77.37 \\
\hline C. owstonii & 0.26 & 0.02 & 1.99 & 0.5 & 2.85 & 80.22 \\
\hline C. squamosus & 0.16 & 0.12 & 1.71 & 0.7 & 2.45 & 82.67 \\
\hline D. calcea & 0.21 & 0.02 & 1.67 & 0.37 & 2.39 & 85.07 \\
\hline $\begin{array}{c}\text { A. sp. cf. } \\
\text { manis }\end{array}$ & 0.26 & 0.01 & 1.55 & 0.54 & 2.22 & 87.29 \\
\hline $\begin{array}{c}\text { A. sp. cf. } \\
\text { albisoma }\end{array}$ & 0.13 & 0 & 1.45 & 0.26 & 2.08 & 89.37 \\
\hline B. naylori & 0.13 & 0.04 & 1.13 & 0.46 & 1.62 & 90.99 \\
\hline
\end{tabular}




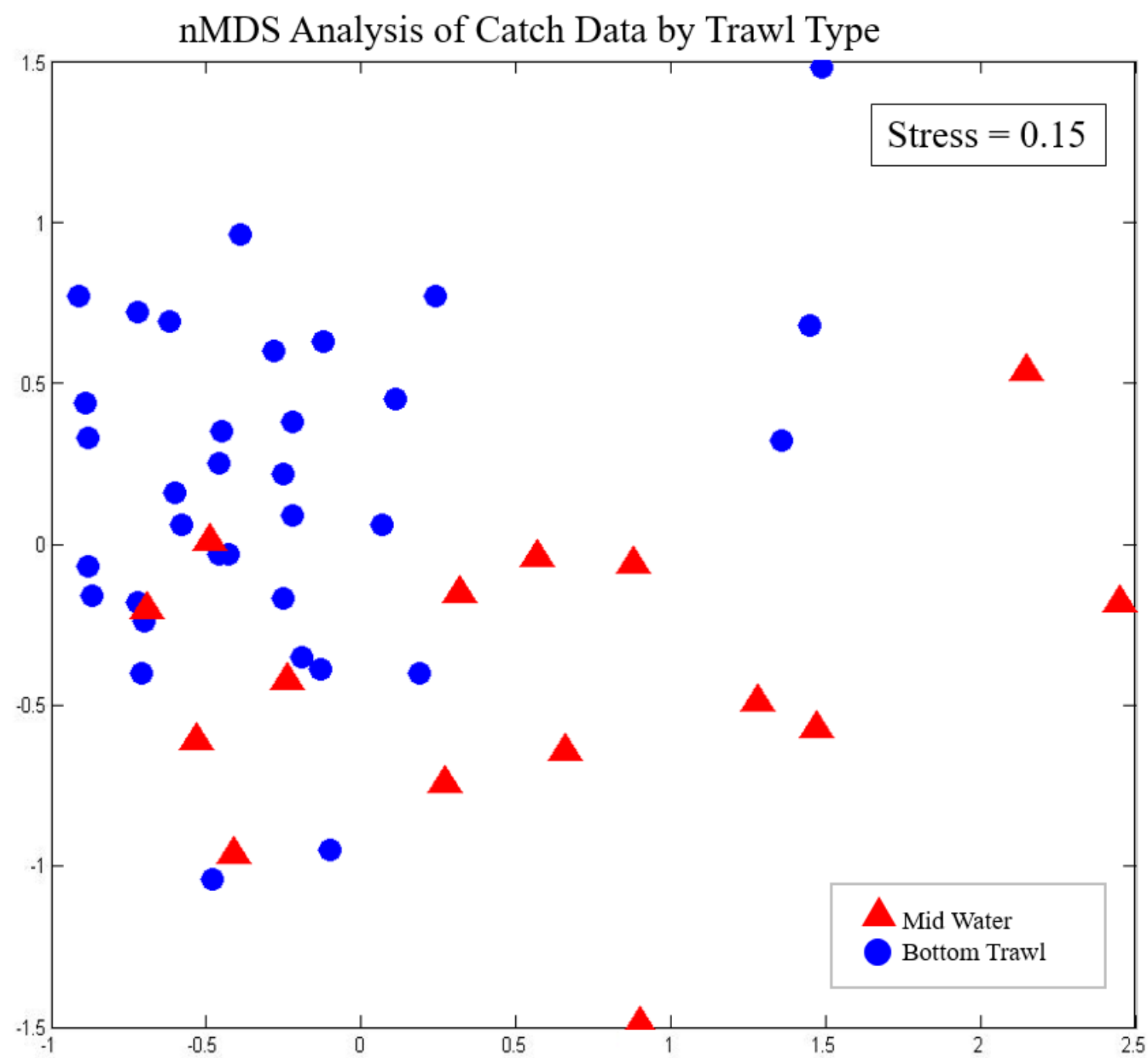

Figure 47. Plot of nMDS analysis illustrating differences in species composition of gear types.

Endemism is common among deep-water species with small bodies and an affinity for the bottom (Kyne and Simpfendorfer, 2010). Although some species encountered are known to be widespread with low site fidelity (E. pusillus, C. crepidater, C. coelolepis, etc.), many of the genera (Etmopterus, Apristurus, Chimaera, and Hydrolagus) are noted for their high degree of endemism (Ebert and Bizzarro, 2007; Didier, 2012; Kyne and Simpfendorfer, 2010). Thirteen species studied during this survey (41.9\% of total) 
demonstrated strong site fidelity and were encountered from a cluster of sites in only one of the two main ecosystems of the region, and in several cases were found at only a single site (Figs. 24, 29, 32, 33, 39, 42, and 44). The low vagility of the region's Chondrichthyan fauna might account for the high Chondrichthyan diversity within the area, since endemism directly correlates with speciation (Musick, et al., 2004; Stevens 2010).

Compared to the Walters Shoal region of the Madagascar Ridge ecosystem, the Southwest Indian Ocean Ridge tended to have more mature females and was the only place pregnant individuals from genera Etmopterus, Centroselachus, and Pseudotriakis were found, potentially indicating its importance as a nursery supplying refuge and/or trophic benefit.

Gravid females are often absent from deep-sea shark surveys, and it has been theorized that pregnant females segregate themselves to pupping grounds or midwater habitats to avoid competition (Ebert, 1994; Kyne and Simpfendorfer, 2010). The presence of females with term embryos and neonates almost exclusively at the Southwest Indian Ocean Ridge suggests this area is a pupping ground for several deep-sea shark species.

Life Strategy and Reproductive Mode. According to life history theory (Williams, 1966), reproductive modes naturally select for an optimum ratio of parental investment to offspring survival (number offspring and likelihood of survival) balanced to best propagate an individual's genes (Hussey et al., 2010). During this survey, four main groups of chondrichthyans were encountered: the squaloid dogfish (18 species), scyliorhinid catsharks (8 species), pseudotriakids (1 species), and holocephalans (4 
species), with the dogfish and catsharks being by far the most commonly observed. These two groups represent vastly different reproductive strategies and associated life histories. The Squaliformes are viviparous, the most common form of reproduction in the shark world (Ebert et al., 2013). The Scyliorhinidae are mostly oviparous, with all Apristurus displaying single egg case oviparity and Bythaelurus displaying either oviparity or viviparity (Francis, 2006b; Ebert et al., 2006; Ebert et al., 2013; Ebert and Clerkin, 2015).

Reproductive modes of this study correlated with major parameters of life history such as sex ratio (statistically significant), sexual dimorphism (statistically significant), and potentially diet and distribution (statistical significance not calculated). Viviparous species had significantly skewed sex ratios favoring females, with females attaining greater total lengths and maturing at a greater size than males, while oviparous species had roughly equal sex ratios, similar total lengths, and similar lengths of maturation. The skewed sex ratios favoring females in viviparous species could represent segregation behavior. Since the viviparous females of this study were larger in size than the males of their species, it is possible that division of habitat is necessary to provide a trophic advantage required to meet the increased energetic demand associated with size.

The correlation between viviparity and relatively larger body size of females compared to males could be explained by the higher caloric requirement and mass compensation to maintain locomotion while hosting a litter for an extended period of time (Grubbs, 2010). Larger female body size could compensate for the burden of hosting pups, and result in greater mobility required to forage for high calorie food items or to 
migrate to nursery areas with refuge and trophic advantages (Grubbs, 2010). Large females with access to high calorie food items could produce larger pups in higher quantity (Hussey et al., 2010). This is contrary to what we commonly observe in terrestrial mammals and birds, where competition between males emphasizes size as the deciding factor for the right to reproduce (Darwin, 1859; Ralls, 1977; Ralls and Mesnick, 2009). In the sparsely populated environment of the deep-sea, males are less limited by direct competition, but more by the ability to find a mate (Rohde, 1991). Although, sexual competition is traditionally thought to take place between males, female body size has been shown to be a competitive character in some vertebrate species (Clutton-Brock, 2009). The defined sexual dimorphism in deep-sea viviparous shark species could be a result of size-selected pressure primarily affecting females, which is absent in single egg case oviparous species. Interestingly, the only oophagous species encountered in this survey, Pseudotriakis microdon, had larger females $(291.2 \mathrm{~cm})$ than males $(233 \mathrm{~cm})$ atthough larger females $(296 \mathrm{~cm})$ and males $(269 \mathrm{~cm})$ have been reported in the literature, Table 9d) - possibly supporting that large females better compensate for the burden of bearing live offspring (Table 9).

In contrast, the oviparous species of this study (all single egg case per uterus) did not segregate spatially by location or depth (Table 14). The majority of the oviparous sex ratios were roughly 1:1, and each sex reached similar size ranges. Previous studies of scyliorhinids have also observed monomorphism between males and females (Compagno, 1984; Cross, 1988; Richardson et al., 2000; Ebert, 2005). However, since our knowledge of sexual dimorphism in marine species is incomplete (Ralls and 
Mesnick, 2009), caution is suggested when inferring evolutionary cause and effect of dimorphic traits in deep-sea sharks.

The complexity and variation of chondrichthyan life histories illustrated in this study demonstrate that teleost and coastal shark management policies are not necessarily directly translatable to deep-sea chondrichthyans. A detailed understanding specifically of the life histories of deep-sea chondrichthyans is necessary in order to create policies to manage our deep-sea resources. 


\section{Synthesis}

The objectives of this study were to clarify the ambiguous taxonomic status of SWIO chimaeroids and catalogue the Chondrichthyan fauna along the northern section of the Madagascar Ridge, Walters Shoal, and the Southwestern Indian Ocean Ridge in order to provide a baseline of life history data.

The family Chimaeridae, despite its global distribution (Ebert and Winton, 2010), remains poorly understood (Didier et al., 2012), and although the SWIO is considered a "hotspot" for chondricthyans, the deep-sea fauna of this region is still poorly known (Ebert, 2014). The taxonomic status of SWIO chimaeroids was virtually unknown with no previous studies confirming the species from seamounts of the offshore region (Ebert, 2014). This study provides the first record of the genus in the Southwestern Indian Ocean, and increases the global total to 23 species, including the three new species described in this study (Clerkin et al., In Press).

In addition to the taxonomic resolution provided for three new Chimaera species, data gathered during these survey cruises contributed to the descriptions of two new catsharks, Bythaelurus bachi and B. naylori (Ebert and Clerkin, 2015; Weigmann et al., 2016), and to the taxonomic resolution of several other taxa. This included the genera Centrophorus, Centroscymnus, Etmopterus, and Scymnodon (Straube et al., 2015; White et al., 2013, 2015; Weigmann et al., 2016). Furthermore, tissue samples collected during these surveys were sent to Dr. Gavin Naylor, College of Charleston, for inclusion into the Tree of Life project (web site), which provides an account of extant chondrichthyan and a 
framework of their relationships through genetic analysis, morphometric, and the fossil record.

The study area was speciose with 31 species spanning 14 genera (Centrophorus, Deania, Etmopterus, Scymnodon, Centroscymnus, Centroselachus, Zameus, Somniosus, Dalatias, Apristurus, Bythaelurus, Pseudotriakis, Chimaera, and Hydrolagus). Biological data was collected to compile an overview of the region's shark fauna. Although life history traits are among the most important parameters with which to evaluate species productivity (Simpfendorfer et al., 2011; White and Last 2012), such data are largely absent for deep-sea sharks in the SWIO, leaving most of our knowledge of chondrichthyans to be based on near shore and pelagic species (Ebert, 2014; Rigby and Simpfendorfer 2014). During this study, a successful census of the chondrichthyan and analysis of data provided an overview of sharks in the understudied region. The surveys spanned 46 sites and over 400 hauls to provide a rudimentary catalogue of species and baseline of population and life history information, but additional, more comprehensive studies are required to better understand the state of SWIO deep-sea sharks and improve information available to policy makers. New species and first accounts taken during this survey not only show how much we have yet to discovery about sharks in the deep ocean, but also highlight the complex variation in life histories among deep-sea shark species. If we are to continue our reliance on the ocean as a source of fish protein, we must acquire information that allows us to predict how groups might respond to harvest, and use that information to formulate sustainable, ecosystem-based management policies. 


\section{Literature Cited}

Acuña-Marrero, D. \& Zimmerhackel, J.S. \& Mayorga, J. \& Hearn, A. (2013) First record of three shark species, Odontaspis ferox, Mustelus albipinnis and Centrophorus squamosus, from the Galápagos Islands. Marine Biodiversity Records, 6: e87 DOI: 10.1017/S1755267213000596

Angulo, A., López, M.I., Bussing, W.A. \& Murase, A. (2014) Records of chimaeroid fishes (Holocephali: Chimaeriformes) from the Pacific coast of Costa Rica, with the description of a new species of Chimera (Chimaeridae) from the eastern Pacific Ocean. Zootaxa, 3861 (6): 554-574.

DOI: 10.11646/zootaxa.3861.6.3.

Barnett, L. A. K., Didier, D.A., Long, D.J. \& Ebert, D.A. (2006) Hydrolagus mccoskeri sp. nov., a new species of chimaeroid fish from the Galápagos Islands (Holocephali:Chimaeriformes: Chimaeridae). Zootaxa, 1328: 27-38.

Barnett, L.A.K. \& Earley, R.L. \& Ebert, D.A. \& Cailliet, G.M. (2009) Maturity, fecundity, and reproductive cycle of the spotted ratfish, Hydrolagus colliei. Marine Biology, 156 (3):301-316

DOI: $10.1007 / \mathrm{s} 00227-008-1084-y$

Bass, A.J., L.J.V. Compagno and P.C. Heemstra, (1986) Squalidae. p. 49-62. In M.M. Smith and P.C. Heemstra (eds.) Smiths' sea fishes. Springer-Verlag, Berlin.

Bauchot, M.-L., (1987) Poissons osseux. p. 891-1421. In W. Fischer, M.L. Bauchot and M. Schneider (eds.) Fiches FAO d'identification pour les besoins de la pêche. (rev. 1). Méditerranée et mer Noire. Zone de pêche 37. Vol. II. Commission des Communautés Européennes and FAO, Rome.

http://dx.doi.org/

Bernardes, R.Á. \& De Figueiredo, J.L. \& Rodrigues, A.R. \& Fischer, L.G. \& Vooren, C.M. \& Haimovici, M. \& Rossi-Wongtschowski, C.L.D.B. (2005) Peixes de Zona Econômica Exclusiva da Região Sudeste-Sul do Brasil: Levantamento com armadilhas, pargueiras e rede de arrasto de fundo. São Paulo: Editora da Universidade de São Paulo: 295 p.

Bianchi, G., K.E. Carpenter, J.-P. Roux, F.J. Molloy, D. Boyer and H.J. Boyer (1999) FAO species identification guide for fishery purposes. Field guide to the living marine resources of Namibia. FAO, Rome. 265 p. 
Bigman, J.S., Ebert, D.A. \& Goldman, K.J. (2016) Squalus suckleyi. The IUCN Red List of Threatened Species 2016: e.T195488A2382480. http://dx.doi.org/10.2305/IUCN.UK.20161.RLTS.T195488A2382480.en. Downloaded on 05 November 2016.

Bizzarro, J.J. \& Broms, K.M. \& Logsdon, M.G. \& Ebert, D.A. \& Yoklavich, M.M. \& Kuhnz, L.A. \& Summers, A.P. (2014) Spatial Segregation in Eastern North Pacific Skate Assemblages. PLoS ONE, 9 (10): e109907

Braccini, J.M. \& Chiaramonte, G.E. (2002) Reproductive biology of Psammobatis extenta. Journal of Fish Biology, 61 (1): 272-288 DOI: 10.1111/j.1095-8649.2002.tb01752.x

Bullis, H. R. (1967) Depth segregation and distribution of sex-maturity groups in the Marbled catshark, Galeus arae. In Sharks, Skates and Rays (Gilbert, P. W., Mathewson, R. F. \& Rall, D. P., eds), pp. 141-148. Baltimore, MD: The John Hopkins University Press.

Capapé, C. \& Hemida, F. \& Quignard, J.-P. \& Ben Amor, M.M. \& Reynaud, C. (2008) Biological observations on a rare deep-sea shark, Dalatias licha (Chondrichthyes: Dalatiidae), off the Maghreb coast (south-western Mediterranean). Pan-American Journal of Aquatic Sciences, 3 (3): 355-360

Carlson, J.K. (1999) Occurrence of neonate and juvenile sandbar sharks, Carcharhinusplumbeus, in the northeastern Gulf of Mexico. Fishery Bulletin, 97 (2): 387-391

Carpenter, K.E. \& Niem, V.H. (1998) FAO species identification guide for fishery purposes. The living marine resources of the Western Central Pacific. Volume 2. Cephalopods, crustaceans, holothurians and sharks. Rome, FAO. 1998: 687-1396

Carrier, J.C. \& Pratt, H.L. \& Castro, J.I. (2004) Reproductive biology of elasmobranchs. In: Carrier, J.C. \& Musick, J.A. \& Heithaus, M.R. (eds) Biology of Sharks and their Relatives, Chapter 10: 269-286

Clark, A. M. (1972) Some crinoids from the Indian Ocean. Bulletin of the British Museum (Natural History) 24(2):73-156.

Clark, M. R. (2009). Deep-sea seamount fisheries: a review of global status and future prospects. Latin American Journal of Aquatic Research. 37(3). 
Clarke, M.W. \& Connolly, P.L. \& Bracken, J.J. (2001) Aspects of reproduction of the deepwater sharks Centroscymnus coelolepis and Centrophorus squamosus from west of Ireland and Scotland. Journal of the Marine Biological Association of the United Kingdom, 81 (6): 1019-1029

DOI: $10.1017 / S 0025315401005008$

Clarke, M.W. \& Connolly, P.L. \& Bracken, J.J. (2002) Catch, discarding, age estimation, growth and maturity of the squalid shark Deania calceus west and north of Ireland. Fisheries Research, 56 (2): 139-153

DOI: 10.1016/S0165-7836(01)00419-2

Claro, R. (1994) Características generales de la ictiofauna. In: R. Claro (ed.) Ecología de los peces marinos de Cuba. Instituto de Oceanología Academia de Ciencias de Cuba and Centro de Investigaciones de Quintana Roo: 55-70

Clutton-Brock T. (2009) Sexual selection in females. Animal Behavior 77, 31110.1016/j.anbehav.2008.08.026

DOI: 10.1016/j.anbehav.2008.08.026)

Clerkin, P.J. \& Ebert, D.A. (2017) First southeastern Atlantic record of the false catshark, Pseudotriakis microdon (Carcharhiniformes: Pseudotriakidae). Marine Biodiversity, in press

DOI: $10.1007 / s 12526-016-0594-y$

Clerkin, P.J., Ebert, D.A., Kemper J.M. (2017) New species of Chimaera (Chondrichthyes: Holocephali: Chimaeriformes: Chimaeridae) from the Southwestern Indian Ocean. Zootaxa, in press

Coelho, R. \& Erzini, K. (2005) Length at first maturity of two species of lantern sharks (Etmopterus spinax and Etmopterus pusillus) of southern Portugal. Journal of the Marine Biological Association of the United Kingdom, 85 (5): 1163-1165 DOI: $10.1017 / S 0025315405012245$

Collette, B.B., Russo, J.L., \& Zavala-Camin, L.A. (1978) Scomberomorus brasiliensis, a new species of Spanish mackerel from the western Atlantic. Fishery Bulletin, 76, 273-280.

Collette, B.B. \& Parin, N.V. (1991) Shallow-water fishes of Walters shoals, Madagascar Ridge. Bulletin of Marine Science Vol. 48, No 1, pp. 1-22. 
Collette BB, Carpenter KE, Polidoro BA, J Jorda MJ, Boustany A, Die DJ, Elfes C, Fox W, Graves J, Harrison L, McManus R, Minte-Vera CV, Nelson RJ, Restrepo V, Schratwieser J, Sun C-L, Amorim A, Brick Peres M, Canales C, Cardenas G, Chang S-K, Chiang W C, de Oliveira Leite JN, Harwell H, Lessa R, Fredou FL, Oxenford HA, Serra R, Shao K T, Sumaila R, Wang S-P, Watson R, Yáñez E. (2011) High value and long-lived - double jeopardy for tunas and billfishes. Science 333:291292.

DOI: $10.1126 /$ science.1208730.

Compagno, L.J.V. (1984) FAO Species Catalogue. Vol 4: Sharks of the world, Part 1 Hexanchiformes to Lamniformes. FAO Fisheries Synopsis No. 125, 4 (1): 1-250

Compagno, L.J.V. (1998) Part Sharks: Pristiophoridae, Ginglymostomatidae, Stegostomatidae, Rhincodontidae, Pseudocarchariidae, Alopiidae, Lamnidae, Pseudotriakidae, Hemigaleidae, Sphyrnidae In: K.E. Carpenter and V.H. Niem (eds.), FAO Identification Guide for Fishery Purposes. The Living Marine Resources of the Western Central Pacific. Food and Agriculture Organization, Rome: 1233-1234, 1260-1263, 1268-1278, 1296, 1305-1311, 1361-1366

Compagno, L.J.V. (1990) Alternative life-history styles of cartilaginous fishes in time and space. Environmental Biology of Fishes, 28(1-4): 33-75, figs 1-36. DOI: $10.1007 / \mathrm{BF} 00751027$.

Compagno, L.J.V. (2001) Sharks of the world. An annotated and illustrated catalogue of shark species known to date. Vol. 2. Bullhead, mackerel and carpet sharks (Heterodontiformes, Lamniformes and Orectolobiformes). FAO Spec. Cat. Fish. Purp. 1(2):269p. Rome: FAO.

Compagno, L.J.V. \& Ebert, D.A. \& Smale, M.J. (1989) Guide to the sharks and rays of Southern Africa. Struik, Cape Town, 160 pp.

Compagno, L.J.V. \& Niem, V.H. (1998) Part Sharks: Hexanchidae, Echinorhinidae, Squalidae, Squatinidae, Heterodontidae, Parascyllidae, Bracheluridae, Orectolobidae, Hemiscyllidae, Odontaspididae, Scyliorhinidae, Proscyllidae, Triakidae, In: K.E. Carpenter and V.H. Niem (eds.), FAO Identification Guide for Fishery Purposes. The Living Marine Resources of the Western Central Pacific. Food and Agriculture Organization, Rome: 1195-1232, 1235-1259, 1264-1267, 1279-1295, 1297-1304, 1312-1360

Compagno, L.J.V. \& Stehmann, M. \& Ebert, D.A. (1990) Rhinochimaera africana, a new longnosed chimaera from southern Africa, with comments on the systematics and distribution of the genus Rhinochimaera Garman, 1901 (Chondrichthyes, Chimaeriformes, Rhinochimaeridae). South African Journal of Marine Research, 9: 201222 
Compagno, L.J.V. \& Dando, M. \& Fowler, S. (2005) Sharks of the World. Princeton University Press.

Cortés, E. (1999) Standardized diet compositions and trophic levels of sharks. ICES Journal of Marine Science, 56 (5): 707-717

DOI: 10.1006/jmsc.1999.0489

Cortés, E. (2000) Insights on shark conservation from stochastic demographic modeling. Abstract. American Elasmobranch Society 16th Annual Meeting, 14-20 June 2000, La Paz, Mexico.

Cox, G. \& Francis, M.P. (1997) Sharks and rays of New Zealand. Canterbury University Press, England, $68 \mathrm{pp}$.

Cross, J. N. (1988) Aspects of the biology of two scyliorhinid sharks, Apristurus brunneus and Parmaturus xaniurus, from the upper continental slope off southern California. Fishery Bulletin 86 (4): 691-702.

Daley, R.K. \& Stevens, J.D. \& Graham, K. (2002) Catch analysis and productivity of the deep water dogfish resource in southern Australia. Final Report to FRDC project 1998/108. CSIRO Marine Research 106 pp.

Darwin, C (1859) On the Origin of Species by Means of Natural Selection, Or, the Preservation of Favoured Races in the Struggle for Life. London: J. Murray. Print.

Didier, D.A. (1995) Phylogenetic systematics of extant chimaeroid fishes (Holocephali, Chimaeroidei). American Museum Novitates, 3119: 1-86.

Didier, D.A. (1998) The leopard Chimaera, a new species of chimaeroid fish from New Zealand (Holocephali, Chimaeriformes, Chimaeridae). Ichthyological Research, 45 (3): 281-289.

DOI: http://dx.doi.org/10.1007/BF02673926

Didier, D.A. (2002) Two new species of chimaeroid fishes from the southwestern Pacific Ocean (Holocephali, Chimaeridae). Ichthyological Research, 49 (4): 299-306 DOI: $10.1007 / s 102280200045$

Didier, D.A. \& Leclair, E.E. \& Vanbuskirk, D.R. (1998) Embryonic staging and external features of development of the chimaeroid fish, Callorhinchus milii (Holocephali,Callorhinchidae). Journal of Morphology, 236 (1): 25-47 DOI: 10.1002/(SICI)1097-4687(199804)236:1<25::AID-JMOR2>3.0.CO;2-N 
Didier, D.A. \& Rosenberger, L.J. (2002) The spotted ratfish, Hydrolagus colliei: notes on its biology with a redescription of the species (Holocephali:

Chimaeridae). California Fish and Game, 88 (3): 112-125

Didier, D.A. \& Séret, B. (2002) Chimaeroid fishes of New Caledonia with description of a new species of Hydrolagus (Chondrichthyes, Holocephali). Cybium, 26 (3): 225233.

Didier, D.A., Kemper, J.M. \& Ebert, D.A. (2012) Phylogeny, biology, and classification of extant Holocephalans. In: Carrier, J.C., Musick, J.A. \& Heithaus, M.R. (eds) Biology of Sharks and their Relatives, Edition 2. CRC Press, Boca Raton, Florida: 97-124.

Dulvy, N.K., Notarbartolo di Sciara, G., Serena, F., Tinti, F. \& Ungaro, N., Mancusi, C. \& Ellis, J. (2006) Dipturus batis. The IUCN Red List of Threatened Species. Version 2014.3. <www.iucnredlist.org>. Downloaded on 28 November 2014.

Dulvy, N.K., Fowler, S.L., Musick, J.A., Cavanagh, R.D., Kyne, P.M., Harrison, l.R., Carlson, J.K., Davidson, l.N.K., Fordham, S.V., Francis, M.P., Pollock, C.M., Simpfendorfer, C.A., Burgess, G.H., Carpenter, K.E., Compagno, L.J.V., Ebert, D.A., Gibson, C., Heupel, M.R., Livingstone, S.R., Sanciangco, J.C., Stevens, J.D., Valenti, S. \& White, W.T. (2014) Extinction risk and conservation of the world's sharks and rays. eLife, 3: e00590.

DOI: $10.7554 /$ eLife.00590.

Dunn, M.R. \& Griggs, L. \& Forman, J. \& Horn, P.L. (2010) Feeding habits and niche separation among the deep-sea chimaeroid fishes Harriotta raleighana, Hydrolagus bemisi and Hydrolagus novaezealandiae. Marine Ecology Progress Series, 407: 209-225

DOI: $10.3354 /$ meps08580

Ebert, D.A. (1986) Biological aspects of the sixgill shark, Hexanchus griseus. Copeia, 1986 (1): 131-135

Ebert, D.A. (1994) Diet of the sixgill shark Hexanchus griseus of southern Africa. South African Journal of Marine Science, 14 (1): 213-218

DOI: $10.2989 / 025776194784287030$

Ebert, D.A. (1996) Biology of the sevengill shark Notorynchus cepedianus (Peron, 1807) in the temperate coastal waters of southern Africa. South African Journal of Marine Science, 17 (1): 93-103

DOI: $10.2989 / 025776196784158545$ 
Ebert, D. A. (2003). The Sharks, Rays and Chimaeras of California. Berkeley, CA: University of California Press.

Ebert, D.A. (2005) Reproductive biology of skates, Bathyraja (Ishiyama) along the eastern Bering Sea continental slope. Journal of Fish Biology, 66 (3): 618-649 DOI: $10.1111 / j .0022-1112.2005 .00628 . x$

Ebert, D.A. (2013) Deep-sea Cartilaginous Fishes of the Indian Ocean. Volume 1. Sharks FAO Species Catalogue for Fishery Purposes. No. 8, Vol. 1. Rome.

Ebert, D.A. (2014) Deep-sea Cartilaginous Fishes of the Indian Ocean. Volume 2. Batoids and Chimaeras. FAO Species Catalogue for Fishery Purposes. No. 8, Vol. 2. Rome, FAO. 129 pp.

Ebert, D.A. (2015) Deep-Sea Cartilaginous Fishes of the Southeastern Atlantic Ocean. FAO Species Catalogue for Fishery Purposes No. 9, 264 p.

Ebert, D.A. \& Cowley, P.D. \& Compagno, L.J.V. (1991) A preliminary investigation of the feeding ecology of skates (Batoidea: Rajidae) off the west coast of southern Africa. South African Journal of Marine Science, 10 (1): 71-81 DOI: 10.2989/02577619109504621

Ebert, D.A. \& Compagno, L.J.V. \& Cowley, P.D. (1992) A preliminary investigation of the feeding ecology of squaloid sharks off the West Coast of Southern Africa. South African Journal of Marine Science, 12 (1): 601-609 DOI: $10.2989 / 02577619209504727$

Ebert, D.A. \& Compagno, L.J.V. \& Cowley, P.D. (2006) Reproductive biology of catsharks (Chondichthyes: Scyliorhinidae) off the west coast of southern Africa. ICES Journal of Marine Science, 63: 1053-1065

DOI: 10.1016/j.icesjms.2006.04.016

Ebert, D.A., \& Bizzarro, J.J. (2007) Standardized diet compositions and trophic levels of skates (Chondrichthyes: Rajiformes: Rajoidei). Environmental Biology of Fishes. 80(2/3): 221-237.

Ebert, D.A. \& Winton, M.V. (2010) Chondrichthyans of High Latitude Seas. In: Carrier, J.C. \& Musick, J.A. \& Heithaus, M.R. (Eds) Sharks and Their Relatives II: Biodiversity, Adaptive Physiology, and Conservation. CRC Press, Boca Raton: 115158. 
Ebert, D.A. \& Compagno, L.J.V. \& De Vries, M.J. (2011) A New Lanternshark (Squaliformes: Etmopteridae: Etmopterus) from Southern Africa. Copeia, 2011 (3): 379-384

DOI: 10.1643/CI-09-183

Ebert, D. A., Fowler, S. \& Compagno, L. J. V. (2013) Sharks of the World - A fully illustrated guide. Wild Nature Press, ISBN 978-0-9573946-0-5:528pp.

Ebert, D.A. \& Stehmann, M. (2013) Sharks, batoids, and chimaeras of the North Atlantic. FAO Species Catalogue for Fishery Purposes, 7. Rome, FAO, 523 pp.

Ebert, D.A. \& White, W.T. \& HO, H.C. \& Last, P.R. \& Nakaya, K. \& Seret, B. \& Straube, N. \& Naylor, G.J.P. \& De Carvalho, M.R. (2013). An annotated checklist of the chondrichthyans of Taiwan. Zootaxa, 3752: 279-386

DOI: 10.11646/zootaxa.3752.1.17

Ebert, D.A. \& Knuckey, J.D.S. \& Kamikawa, D.J. (2014) First eastern North Pacific record of the velvet dogfish, Zameus squamulosus (Chondrichthyes: Squaliformes: Somniosidae). Marine Biodiversity Records, 7: e48

DOI: $10.1017 / \mathrm{S} 1755267214000499$

Ebert, D.A. \& Clerkin, P.J. (2015) A new species of deep-sea catshark (Scyliorhinidae:Bythaelurus) from the southwestern Indian Ocean. Journal of the Ocean Science Foundation, 15: 53-63

Ebert, D.A. \& Van Hees, K.E. (2015) Beyond Jaws: rediscovering the 'lost sharks' of southern Africa. African Journal of Marine Science, 37 (2): 141-156

DOI: $10.2989 / 1814232 X .2015 .1048730$

Ebert, D.A. \& Straube, N. \& Leslie, R.W. \& Weigmann, S. (2016) Etmopterus alphus n. sp.: a new lanternshark (Squaliformes: Etmopteridae) from the south-western Indian Ocean. African Journal of Marine Science, 38 (3): 329-340

DOI: $10.2989 / 1814232 X .2016 .1198275$

Eschmeyer, W.N. (2016) Catalog of fishes: Genera, Species, References. Available from: http://research.calacademy.org/research/ichthyology/catalog/fishcatmain.asp (accessed 10 September 2016).

Eschmeyer WN, Fong JD (2014) Species by family/subfamily. Catalog of Fishes electronic version accessed March 2014 http:/research.calacademy.org/research/ichthyology/catalog/SpeciesByFamily.asp 
Eshmeyer, W.N. R. Fricke, R. van der Laan (2017) Catalog of fishes: Genera, Species, References. Available from:

http://research.calacademy.org/research/ichthyology/catalog/fishcatmain.asp (accessed 19 January 2017)

FAO (2012) The State of World Fisheries and Aquaculture. Food and agriculture Organization of the United Nations, Rome. ISBN 978-92-5-107225-7.

FAO (2010-2014) International Plan of Action for the Conservation and Management of Sharks - Web site. International Plan of Action for Conservation and Management of Sharks. FI Institutional Websites. In: FAO Fisheries and Aquaculture Department [online]. Rome. Updated. [Cited 12 November 2014]. http://www.fao.org/fishery/ipoa-sharks/en

Francis, M.P. (2006) Morphometric minefields - towards a measurement standard for chondrichthyan fishes. Environmental Biology of Fishes, 77 (3-4): 407-421 DOI: $10.1007 / \mathrm{s} 10641-006-9109-1$

Francis, M.P. (2006) Distribution and biology of the New Zealand endemic catshark, Halaelurus dawsoni. Environmental Biology of Fishes, 75, 295-306.

Gage, J.D., Tyler, P.A (1991) Deep-Sea Biology: A Natural History of Organisms at the Deep-Sea Floor. Cambridge University Press, Cambridge New York. Xvi + 504 p.; ill.; species and subject indexes.

ISBN: 0-52103343104.

García, V.B. Lucifora, L.O. \& Myers, R.A. (2008) The importance of habitat and lifehistory to extinction risk in sharks, skates, rays and chimaeras. Proceedings of the Royal Society of London, Series B, 275: 83-89.

DOI: $10.1098 / \mathrm{rspb} .2007 .1295$.

Garrick, J.A.F. (1959a) Studies on New Zealand Elasmobranchii. Part IX. Scymnodon plunketi (Waite, 1910), an abundant deep-water shark of New Zealand waters. Transactions of the Royal Society of New Zealand, 87 (3-4): 271-282.

Garrick, J.A.F. (1959b) Studies on New Zealand Elasmobranchii. Part VIII. Two northern hemisphere species of Centroscymnus in New Zealand waters. Transactions of the Royal Society of New Zealand, 87 (1): 75-89.

Gibbs, R.H. Jr. and B.B. Collette, (1967) Comparative anatomy and systematics of the tunas, genus Thunnus. Fishery Bulletin 66(1):65-130. 
Gilchrist, J.D.F. (1922) Deep-sea fishes procured by the S.S. Pickle. Part 1. Report Fisheries and Marine Biological Survey, Union of South Africa, 2 (3), 41-79, Pls. 7 12 .

Gill, T. (1862) Note on some genera of fishes of western North America. Proceedings of the Academy of Natural Sciences of Philadelphia, 14: 329-332

Girard, M. and M.H. Du Buit, 1999. Reproductive biology of two deep-water sharks from the British Isles, Centroscymnus coelolepis and Centrophorus squamosus. Journal of the Marine Biological Association UK 79(5):923-931.

DOI: http://dx.doi.org/

Goslin, J., Segiufin, J., Schlich, R., \& Fisher., R. L. (1980) Submarine Topography and shallow structure of the Madagascar Ridge, western Indian Ocean. Geological Society of America Bulletin 1980; 91, no. 12;741-753.

Griffiths, A.M. \& Sims, D.W. \& Cotterell, S.P. \& El.Nagar, A. \& Ellis, J.R. \& Lynghammar, A. \& Mchugh, M. \& Neat, F.C. \& Pade, N.G. \& Queiroz, N. \& SerraPereira, B. \& Rapp, T. \& Wearmouth, V.J. \& Genner, M.J. (2010) Molecular markers reveal spatially segregated cryptic species in a critically endangered fish, the common skate (Dipturus batis). Proceedings of the Royal Society of London, Series B, 277: 1497-1503

DOI: 10.1098/rspb.2009.2111.

Grubbs, R.D. (2010) Ontogenetic Shifts in Movements and Habitat Use. In: Carrier, J.C. \& Musick, J.A. \& Heithaus, M.R. (Eds) Sharks and Their Relatives II: Biodiversity, Adaptive Physiology, and Conservation. CRC Press, Boca Raton: 319-350

Gubanov, E.P. (1985) Presence of the sharp tooth sand shark, Odontaspis ferox (Odontaspididae), in the open waters of the Indian Ocean. Journal of Ichthyology, 25 (2): 156-158.

Gubanov, E.P. (1988) Morphological characteristics of the requiem shark, Carcharinus obscurus, of the Indian Ocean. Journal of Ichthyology, 28 (6): 68-73.

Haedrich, RL, Merrett, N.R., \& O’Dea, N.R. (2001) Can ecological knowledge catch up with deep-water fishing? A North Atlantic perspective. Fisheries Research 51:113122.

Hardy, G.S. \& Stehmann, M. (1990) A new deep-water ghost shark, Hydrolagus pallidus n. sp. (Holocephali, Chimaeridae), from the Eastern North Atlantic, and redescription of Hydrolagus affinis (Brito Capello, 1867). Archiv für Fischereiwissenschaft, 40 (3), 229-248. 
Hearn, A. \& Ketchum, J. \& Klimley, A.P. \& Espinoza, E. \& Penaherrera, C. (2010) Hotspots within hotspots? Hammerhead shark movements around Wolf Island, Galapagos Marine Reserve Marine Biology, 157 (9): 1899-1915.

DOI: $10.1007 / s 00227-010-1460-2$

Heithaus, M.R. (eds) Biology of Sharks and their Relatives, $2^{\text {nd }}$ Edition. CRC Press, Boca Raton, Florida, pp 97-124

Heupel, M.R. \& Simpfendorfer, C.A. (2010) Science or Slaughter: Need for Lethal Sampling of Sharks. Conservation Biology, 24 (5): 1212-1218.

DOI: 10.1111/j.1523-1739.2010.01491.x

Hoenig, J.M. \& Gruber, S.H. (1990). Life-History Patterns in the Elasmobranchs: Implications for Fisheries Management. In H.L. Pratt, S.H. Gruber, and T. Taniuchi (eds.), Elasmobranchs as Living Resources: Advances in the Biology, Ecology, Systematics and the Status of the Fisheries. US Dept. Comm. NOAA Tech. Rep. NMFS 90:16.

Holden, M.J. (1974) Problems in the rational exploitation of elasmobranch populations and some suggested solutions. In Sea Fisheries Research (Harden-Jones, F. R., Ed.), pp. 117-137. New York: John Wiley \& Sons.

Holden, M.J. (1975) The fecundity of Raja clavata in British waters. Journal du Conseil International pour I'Exploration de la Mer, 36 (2): 110-118

DOI: 10.1093/icesjms/36.2.110

Hubbs, C. L. \& Ishiyama, R. (1968) Methods for the taxonomic study and description of skates (Rajidae). Copeia 1968, 483-491.

Hussey, N.E. \& Wintner, S.P. \& Dudley, S.F.J. \& Cliff, G. \& Cocks, D.T. \& MacNeil, M.A. (2010) Maternal investment and size-specific reproductive output in carcharhinid sharks. Journal of Animal Ecology, 79 (1): 184-193

DOI: 10.1111/j.1365-2656.2009.01623.x

Hutchings, J.A. \& Myers, R.A. \& García, V.B. \& Lucifora, L.O. \& Kuparinen, A. (2012) Life history correlates of extinction risk and recovery potential. Ecological Applications, 22 (4): 1061-1067

DOI: $10.1890 / 11-1313.1$

Iglésias, S.P. \& Toulhoat, L. \& Sellos, D.Y. (2010) Taxonomic confusion and market mislabelling of threatened skates: important consequences for their conservation status. Aquatic Conservation: Marine and Freshwater Ecosystems, 20: 319-333 DOI: 10.1002/aqc.1083. 
Inada, T. \& Garrick, J.A.F. (1979) Rhinochimaera pacifica, a long-snouted Chimaera (Rhinochimaeridae), in New Zealand waters. Japanese Journal of Ichthyology, 25 (4): $235-243$

Jackson JBC, (2010) The future of the oceans past. Philosophical Transactions of the Royal Society B: Biological Sciences 365:3765-3778.

DOI: 10.1098/rstb.2010.0278.

Jawad, L.A. \& Al-Rassady, I. \& Al-Mamry, J.M. (2013) Five new records of fishes from the Arabian Sea coasts of Oman. Marine Biodiversity Records, 6: e29 DOI: $10.1017 / S 1755267213000122$

Jordan, D.S. \& Snyder, J.O. (1900) A list of fishes collected in Japan by Keinosuke Otaki, and by the United States Streamer Albatross, with descriptions of fourteen new species. Proceedings of the United States National Museum, 23, 335-380, 12 pls.

Kemper, J.M. \& Ebert, D.A. \& Compagno, L.J.V. \& Didier, D.A. (2010a) Chimaera notafricana, sp. nov. (Chondrichthyes: Chimaeriformes: Chimaeridae), a new species of chimaera from southern Africa. Zootaxa, 2532, 55-63

Kemper, J.M. \& Ebert, D.A. \& Didier, D.A. \& Compagno, L.J.V. (2010b) Description of a new species of chimaerid, Chimaera bahamaensis from the Bahamas (Holocephali: Chimaeridae). Bulletin of Marine Science, 86 (3), 649-659.

Kemper, J.M. \& Ebert, D.A. \& Naylor, G.J.P. \& Didier, D.A. (2015) Chimaera carophila (Chondrichthyes: Chimaeriformes: Chimaeridae), a new species of chimaera from New Zealand. Bulletin of Marine Science, 91 (1), 63-81. DOI: http:/dx.doi.org/10.1007/s00227-010-1460-210.5343/bms.2014.1042

Kensley, B. (1975) Five species of Jaeropsis from the southern Indian Ocean (Crustacea, Isopoda, Asellota). Annals of the South African Museum 67(10): 367-380.

Ketchen, K.S. (1972) Size at maturity, fecundity, and embryonic growth of the spiny dogfish (Squalus acanthias) in British Columbia water. Journal Fisheries Research Board Canada, 29, 1717-1723.

Kyne, P.M. \& Simpfendorfer, C.A. (2007) A Collation and summarization of available data on deepwater Chondrichthyans: Biodiversity, life history and fisheries. Biodiversity, Life History and Fisheries IUCN SSC Shark Specialist Group for the Marine Conservation Biology Institute, 1-137. 
Kyne, P.M. \& Simpfendorfer C.A. (2010) Deepwater chondrichthyans. In: Carrier, J.C., Musick, J.A. \& Heithaus, M.R. (Eds) Sharks and Their Relatives II: Biodiversity, Adaptive Physiology, and Conservation. CRC Press, Boca Raton: 37-114.

Lamilla, J. \& Sáez, S. (2003) Clave taxonómica para el reconocimiento de especies de rayas chilenas (Chondrichthyes, Batoidei). Investigaciones Marinas, 31 (2): 3-16 DOI: $\underline{10.4067 / S 0717-71782003000200001}$

Last, P.R. (2007) The state of chondrichthyan taxonomy and systematics. Marine and Freshwater Research, 58 (1): 7-9.

DOI: 10.1071/MF07003.

Last, P.R. \& Stevens, J.D. (1994) Sharks and Rays of Australia. CSIRO, Australia, pp. iix, 1 513, 1400 black and white ill., colour pls. 1-84

Last, P.R. \& Stevens, J.D. (2009) Sharks and Rays of Australia. CSIRO Publishing, Collingwood, Vic.

Last, P.R. \& De Carvalho, M.R. \& Naylor, G.J.P. \& Séret, B. \& Stehmann, M.F.W. \& White, W.T. (2016) Introduction. In: Last, P.R., White, W.T., Carvalho, M.R. de, Séret, B., Stehmann, M.F.W \& Naylor, G.J.P (Eds.) Rays of the World. CSIRO Publishing, Melbourne: 1-9

Mabragaña, E., Lucifora L. O. \& Massa, A. M. (2002) The reproductive ecology and abundance of Sympterygia bonapartii endemic to the south-west Atlantic. Journal of Fish Biology 60, 951-967.

DOI: 10.1006/jfbi. 2002.1903

Macpherson, E. (1989) Influence of geographical distribution, body size and diet on population density of benthic fishes off Namibia (South West Africa). Marine Ecology Progress Series, 50: 295-299

McClenachan. L., Cooper A.B., Carpenter K.E., Dulvy N.K., (2012) Extinction risk and bottlenecks in the conservation of charismatic marine species. Conservation Letters 5:73-80.

DOI: 10.1111/j.1755-263X.2011.00206.x.

Mccosker, J.E. \& Long, D.J. \& Baldwin, C.C. (2012) Description of a New Species of Deepwater Catshark, Bythaelurus giddingsi sp. nov., from the Galápagos Islands (Chondrichthyes: Carcharhiniformes: Scyliorhinidae). Zootaxa, 3221: 48-59

Mollet, H.F. \& Cliff, G. \& Pratt, H.L. \& Stevens, J.D. (2000) Reproductive biology of the female shortfin mako, Isurus oxyrinchus Rafinesque, 1810, with comments on the embryonic development of lamnoids. Fishery Bulletin, 98 (2): 299-318, figs 1-6 
Morato, T., Watson, R., Pitcher, T.J. \& Pauly, D. (2006) Fishing down the deep. Fish and Fisheries, 7:24-34.

Mundy, B.C. (2005) Checklist of the fishes of the Hawaiian Archipelago. Bishop Museum Bulletin in Zoology, 6: 1-704

Musick J.A., Harbin M.M., Compagno L.J.V. (2004) Historical zoogeography of the Selachii. In: Carrier JF, Musick JA, Heithaus MR (eds) Biology of sharks and their relatives. BocaRaton, FL: CRC Press, pp 33-78.

Musick, J.A. \& Ellis, J.K. (2005) Reproductive evolution of chondrichthyans. In: Hamlett, W.C. (ed.) Reproductive biology and phylogeny of chondrichthyes: sharks, rays and chimaeras, Vol. 3. Endfield, USA: Science Publishers: 45-79

Nakaya, K. \& Sato, K. \& Iglésias, S.P. \& White, W.T. (2008) Methodology for the taxonomic description of members of the genus Apristurus (Chondrichthyes: Carcharhiniformes: Scyliorhinidae). CSIRO Marine and Atmospheric Research Paper, 22: 49-60

Nelson, J. S. (2016) Fishes of the world (5th edition). John Wiley and Sons, Inc., Hoboken, New Jersey.

Neer, J. A. \& Cailliet, G. M. (2001) Aspects of the life history of the Pacific electric ray, Torpedo californica (Ayres). Copeia 2001, 842-847.

Novikov, N.P. (2002) Ecology of the ratfish Hydrolagus africanus (Gilchrist) from the Madagascar and Mozambique submarine ridges. Journal of Ichthyology, 42 (3), 271-274.

Peres, M.B. \& Vooren, C. M. (1991) Sexual development, reproductive cycle, and fecundity of the school shark Galeorhinus galeus off southern Brazil. Fishery Bulletin 89, 655-667.

Ralls, K., (1977) Sexual dimorphism in mammals: avian models and unanswered questions The American Naturalist 111 (981), 917-938

Ralls K, Mesnick S (2009) Sexual dimorphism. In: Perrin WF, Wursig B, Thewissen JGM (eds) Encyclopedia of Marine Mammals. 2nd eds. Academic Press, San Diego, pp 1005-1011 
Richardson, A.J. \& Maharaj, G. \& Compagno, L.J.V. \& Leslie, R.W. \& Ebert, D.A. \& Gibbons, M.J. (2000) Abundance, distribution, morphometrics, reproduction and diet of the Izak catshark. Journal of Fish Biology, 56 (3): 552-576

DOI: 10.1111/j.1095-8649.2000.tb00755.x

Rigby, C. \& Simpfendorfer, C.A. (2014) Patterns in life-history traits of deep-water chondrichthyans. Deep Sea Research Part II: Topical Studies in Oceanography DOI: http://dx.doi.org/10.1016/j.dsr2.2013.09.004

Roa, R., Ernst, B. \& Tapia, F. (1999) Estimation of size at sexual maturity: an evaluation of analytical and resampling procedures. Fishery Bulletin 97, 570-580.

Robison, B.H. 2009. Conservation of deep pelagic biodiversity. Conservation Biology 23:847-858.

Rohde, K, (1991) Intra- and interspecific interactions in low density populations in resource-rich habitats. Oikos, 60:91-104.

Romanov, E.V. (2003) Summary and Review of Soviet and Ukrainian Scientific and Commercial Fishing Operations on the Deepwater Ridges of the Southern Indian Ocean. FAO Fisheries Circular No. 991.

Sabaj M.H. (2016) Standard symbolic codes for institutional resource collections in herpetology and ichthyology: an Online Reference. Version 6.5 (16 August 2016). Electronically accessible at http://www.asih.org/, American Society of Ichthyologists and Herpetologists, Washington, DC.

Shirai, S. \& Tachikawa, H. (1993) Taxonomic resolution of the Etmopterus pusillus species group (Elasmobranchii, Etmopteridae), with description of E. bigelowi, n. sp. Copeia, 1993 (2): 483-495

Simpfendorfer, C.A. \& Kyne, P.M. (2009) Limited potential to recover from overfishing raises concerns for deep-sea sharks, rays and chimaeras. Environmental Conservation, 36 (2):97-103.

DOI: $10.1017 / S 0376892909990191$.

Simpfendorfer, C.A., Heupel, M.R., White, W.T. \& Dulvy, N.K. (2011) The importance of research and public opinion to conservation management of sharks and rays: a synthesis. Marine and Freshwater Research, 62 (6): 518-527.

DOI: http://dx.doi.org/10.1071/MF11086

Sinha, M. C., Louden, K. E., \& Parsons, B. (1981) The crustal structure of the Madagascar Ridge. Geophysical Journal of the Royal Astronomical Society, 66, 351-377. 
Soto, J.M.R. (2001) First record of southern lanternshark, Etmopterus granulosus (Günther, 1880) (Squaliformes, Dalatiidae), in Brazilian coast. Mare Magnum, 1 (1): 7-10

Springer, S. (1967) Social organization of shark populations. In Sharks, Skates and Rays (Gilbert, P. W., Mathewson, R. F. \& Rall, D. P., eds), pp. 149-174. Baltimore, MD: The John Hopkins University Press.

Springer, S. (1991) Chlamydoselachidae (p. 2), Squalidae (p. 7-19), Oxynotidae (p. 2021), Squatinidae (p. 77-78), Odontaspididae (p. 81-82), Hemigaleidae (p. 100), Carcharinidae (p. 101-108), In: Quero, J.-C., Hureau, J.C., Karrer, C., Post, A. \& Saldanha, L. (Eds): Check-List Of The Fishes Of The Eastern Tropical Atlantic (Clofeta), vol. 1:xxxii+519 pp. UNESCO, SEI, JNICT-Portugal (1990) 1991 (3 vols).

Stamatakis, A. (2014) RAxML version 8: a tool for phylogenetic analysis and postanalysis of large phylogenies. Bioinformatics, 30 (9), 1312-1313.

Stevens, J.D.R., Bonfil, R., Dulvy, N.K., \& Walker, P.A., (2000) The effects of fishing on sharks, rays, and chimaeras (chondrichthyans), and the implications for marine ecosystems. ICES Journal of Marine Science 57, 476-494.

Stevens, J.D. (2010) Epipelagic Oceanic Elasmobranchs. In: Carrier, J.C. \& Musick, J.A. \& Heithaus, M.R. (Eds) Sharks and Their Relatives II: Biodiversity, Adaptive Physiology, and Conservation. CRC Press, Boca Raton: 3-36.

Straube, N., Leslie, R.W., Clerkin, P.J., Ebert, D.A., Rochel, E., Corrigan, S., Li, C. \& Naylor, G.J.P. (2015) On the occurrence of the southern Lanternshark, Etmopterus granulosus, off South Africa, with comments on the validity of E. compagnoi. Deep Sea Research Part II: Topical Studies in Oceanography. DOI: 10.1016/j.dsr2.2014.04.004

Tanaka, S. (1905) On two new species of Chimaera. Journal of the College of Science, Imperial University, 20 (11), 1-14, Pls. 1-2.

Walker, P. A. \& Hislop, J. R. G. (1998) Sensitive skates or resilient rays? Spatial and temporal shifts in ray species composition in the central and north-western North Sea between 1930 and the present day. ICES Journal of Marine Science 55, 392402. 
Walovich, K.A. \& Ebert, D.A. \& Long, D.J. \& Didier, D.A. (2015) Redescription of Hydrolagus africanus (Gilchrist, 1922) (Chimaeriformes: Chimaeridae), with a review of southern African chimaeroids and a key to their identification. African Journal of Marine Science, 37 (2), 157-165.

Walovich, K.A. \& Ebert, D.A. \& Kemper, J.M. (2017) Hydrolagus erithacus sp. nov. (Chimaeriformes: Chimaeridae), a new species of chimaerid from the Southeastern Atlantic and southwestern Indian oceans. Zootaxa, 4226 (4): 509-520. DOI: http:/dx.doi.org/10.11646/zootaxa.4226.4.4

Weigmann, S. \& Ebert, D.A. \& Clerkin, P.J. \& Stehmann, M.F.W. \& Naylor, G.J.P. (2016) Bythaelurus bachi n. sp., a new deep-water catshark (Carcharhiniformes, Scyliorhinidae) from the southwestern Indian Ocean, with a review of Bythaelurus species and a key to their identification. Zootaxa, 4208 (5): 401-432

Weigmann, S. (2016) Annotated checklist of the living sharks, batoids and chimaeras (Chondrichthyes) of the world, with a focus on biogeographical diversity. Journal of Fish Biology, 88 (3): 837-1037

DOI: $10.1111 / \mathrm{jfb} .12874$

Wetherbee, B.M. (1996) Distribution and reproduction of the southern lantern shark from New Zealand. Journal of Fish Biology, 49 (6): 1186-1196

DOI: 10.1111/j.1095-8649.1996.tb01788.x

Wetherbee, B.M. \& Gruber, S.H. \& Cortés, E. (1990) Diet, feeding habits, digestion and consumption in sharks, with special reference to the lemon shark Negaprionbrevirostris. In: Pratt, H.L., Gruber, S.H., Taniuchi, T., editors. Elasmobranchs as living resources: advances in the biology, ecology, systematics, and the status of the fisheries: NOAA Technical Report NMFS, 90: 29-47

White, W.T. \& Last, P.R. \& Stevens, J.D. \& Yearsley, G.K. \& Fahmi, D. \& Dharmadi (2006) Economically important sharks and rays of Indonesia. ACIAR Monograph series, 124: 1329

White, W.T. \& Kyne, P.M. (2010) The status of chondrichthyan conservation in the Indo Australasian region. Journal of Fish Biology, 76 (9): 2090-2117

DOI: 10.1111/j.1095-8649.2010.02654.x

White, W.T. \& Last, P.R. (2012) A review of the taxonomy of chondrichthyan fishes: a modern perspective. Journal of Fish Biology, 80 (5): 901-917.

DOI: 10.1111/j.1095-8649.2011.03192.x 
White, W.T., Ebert, D.A., Naylor, G.J.P., Ho, H.C., Clerkin, P., Veríssimo, A. \& Cotton, C.F. (2013) Revision of the genus Centrophorus (Squaliformes: Centrophoridae): Part 1 Redescription of Centrophorus granulosus (Bloch \& Schneider), a senior synonym of C. acus Garman and C. niaukang Teng. Zootaxa, 3752: 35-72.

DOI: 10.11646/zootaxa.3752.1.5.

Williams, G. (1966) Natural selection, the costs of reproduction and a refinement of Lack’s principal. American Naturalist, 100, 687-690.

William K. Purves, Gordon H. Orians, David Sadava, H. Craig Heller, Craig Heller (2003). Life: The Science of Biology (7th ed.), pp. 823-824.

Yano K (1992) Comments on the reproductive mode of the false cat shark Pseudotriakismicrodon. Copeia 1992(2):460-468

Yano, K. \& Tanaka, Sho (1987) Reproductive organs of deep sea sharks, Centroscymnusowstonii and C. coelolepis. Journal of the Faculty of Marine Sciences and Technology, Tokai University, 25: 57-67.

Yano, K. \& Tanaka, S. (1988) Size at maturity, reproductive cycle, fecundity, and depth segregation of the deep sea squaloid sharks Centroscymnus owstoni and $C$. coelolepis in Suruga Bay, Japan. Nippon Suisan Gakkaishi, 54 (2): 167-174

Yano, K. \& Musick, J.A. (1992) Comparison of morphometrics of Atlantic and Pacific specimens of the false catshark, Pseudotriakis microdon, with notes on stomach contents. Copeia, 1992 (3): 877-886

Zar, J.H. (1996) Biostatistical Analysis. 3rd edn. Prentice Hall: Englewood Cliffs, NJ. 


\section{APPENDICES}

\section{Appendix A}

Diagram of measurements are presented in Figure 2 (lengths and paired fins), Figure 3 (across body, fin interspace, unpaired fin height, margins, and overlap), Figure 4 (head measurements), Figure 5 (canals of head).

Body measurements and their abbreviations are as follows:

Length measurements: total length (TL) is measured as the snout tip to farthest elements of caudal filament; pre-caudal length (PCL), snout tip to origin of dorsal margin of caudal fin; snout to vent length (SVL), distal tip of snout to cloacal opening; tail length (TLL), cloacal opening to dorsal origin of caudal fin; body length (BDL), dorsal edge of gill opening to origin of dorsal margin of caudal fin; trunk length (TRL), ventral edge of gill opening to cloaca; head length (HDL), snout tip to the dorsal opening of the gill, prefirst dorsal fin length (PD1), snout tip to origin of dorsal fin spine; pre-second dorsal fin length (PD2), snout tip to origin of second dorsal fin; pre-pectoral fin length (PP1), snout tip to origin of pectoral fin anterior margin radials; pre-pelvic fin length (PP2), snout tip to origin of pelvic fin anterior margin radials.

Head measurements: pre-orbital length (POB), snout tip to anterior edge of orbit ; preorbital distance (POBD), snout tip to anterior edge of orbit; prenarial length (PRN), snout tip to anterior edge of nasal apertures; prenarial distance (PRND), direct distance from snout tip to anterior edge of nasal apertures; pre-oral length (POR), snout tip to end of upper labial fold; pre-oral distance (PORD), direct distance snout tip to anterior edge of 
mouth; snout length (SNL), snout tip to oronasal; eye length (EYL), greatest anterior to posterior length across eye; eye height $(\mathrm{EYH})$, greatest dorsal to ventral height across eye.

Cross body measurements: first dorsal to pectoral (D1P1), anterior edge of first dorsal fin base to anterior edge of pectoral-fin base; first dorsal to pelvic (D1P2), anterior edge of base of first dorsal-fin to anterior edge of pelvic-fin base; Second dorsal to pectoral (D2P1), anterior edge of second dorsal-fin base to anterior edge of pectoral-fin base; second dorsal to pelvic (D2P2), anterior edge of second dorsal-fin base to anterior edge of pelvic-fin base.

Width measurements: snout width at base (SWB); snout anterior width (SWF), maximum snout width at distal end of nasal canal; head width at suborbital ridge (SOW), greatest width of head at suborbital ridge; trunk width (TRW), width at pectoral fin insertions; abdominal width (ABW), width at anterior pelvic girdle; tail width (TLW), width at directly posterior of pelvic girdle; caudal peduncle width (CPW), width at caudal peduncle.

Height measurements: snout height at base (SHB), Snout height at base (apex); Head height (HDH), height at dorsal opening of the gill; trunk height (TRH), trunk height at pectoral fin insert; max body height $(\mathrm{MBH})$, maximum depth across trunk; abdomen height; tail height (TLH), height at insertion of pelvic fins; caudal peduncle height $(\mathrm{CPH})$, height at insertion of second dorsal fin.

Inter-fin spaces: interdorsal space (IDS), space between first and second dorsal fins; dorsal-caudal space (DCS), space between second dorsal fin and anterior origin of caudal 
fin; pectoral-pelvic space (PPS), posterior base of pectoral fin insertion to anterior base of pelvic fin origin; pelvic-anal space (PAS), posterior base of pelvic fin to origin of anal fin; Pelvic-caudal space (PCS), pelvic fin insertion to origin of ventral caudal fin; pelvicridge space (PRS), pelvic fin insertion to origin fleshy ridge.

Fin measurements: pectoral fin anterior margin (P1AM), length of the pectoral anterior margin; pectoral fin width (P1FW), maximum width across pectoral fin perpendicular to the anterior margin; pectoral fin base width (P1BW), width of pectoral fin base from origin of anterior margin to insertion of inner margin; pectoral fin base height (P1BH), height of pectoral fin base from body to farthest edge of fin base; pelvic anterior margin (P2AM), length of the pelvic anterior margin; pelvic fin width (P2FW), maximum width across pelvic fin perpendicular to the anterior margin; pelvic fin base width (P2BW), width of pelvic fin base from origin of anterior margin to insertion of inner margin; pelvic fin base height (P2BH), height of pelvic fin base from body to farthest edge of fin base; dorsal spine length (DSA), dorsal spine length along anterior margin; dorsal spine ridge to origin (SRO), dorsal spine width from ridge to origin; first dorsal fin base (D1B), origin of fin spine to insertion of first dorsal fin; first dorsal fin height (D1H), maximum height of first dorsal fin; second dorsal fin base (D2B), origin to insertion of second dorsal fin; second dorsal fin anterior height (D2AH), maximum height of anterior onethird of the second dorsal fin; second dorsal fin posterior height (D2PH), maximum height of posterior one-third of the second dorsal fin; second dorsal fin mid height (D2MH), Lowest point mid second dorsal fin; dorsal caudal margin (CDM), origin to insertion of dorsal caudal fin; dorsal caudal height (CDH), maximum height of dorsal 
lobe of caudal fin; ventral caudal margin (CVM), origin to insertion of ventral caudal fin; fleshy ridge to caudal insertion (RCI), origin of fleshy ridge to insertion of ventral caudal margin; ventral caudal height $(\mathrm{CVH})$, maximum height of ventral lobe of caudal fin; total caudal length (CTL), dorsal origin of caudal fin to end of caudal filament.

Overlap measurements: spine to second dorsal fin (OSD2), the distance which the dorsal spine, when depressed overlaps the origin of the second dorsal fin; pectoral to pelvic (OPP), distance which the distal tips of the pectoral fins overlaps the origin of the pelvic fins; Spine to first dorsal fin (OSD1), the distance which the dorsal spine exceeds the height of the first dorsal fin.

Claspers (males): clasper length total (CLT), total length of claspers from pelvic-fin base to tip; clasper length medial branch (CLM), length of medial branch of clasper from fork to tip; clasper length lateral branch (CLL), length of lateral branch of clasper from fork to tip; clasper length outer (CLO), pelvic fin inner margin to clasper tip; clasper length inner (CLI), inner origin of clasper to distal tip; clasper width at base (CLB), maximum width clasper base; frontal tenaculum length (FTL), length of tenaculum from attachment to body to distal tip; frontal tenaculum bulb height (TBH), bulb height; frontal tenaculum bulb length (TBL), bulb length; frontal tenaculum bulb width (TBW), bulb width; frontal tenaculum stalk width (FTSW), width of stalk.

Postanal pad (females): postanal pad length (APL), postanal pad height (APH), postanal pad width (APW).

Lateral Lines: oronasal to nasal canal (ONC), anterior oronasal fold to center of nasal canal; length of the rostral canal (LRC), length of the rostral canal; length across nasal 
canal (LNC), straight line length across the nasal canal; infraorbital to angular canal (IOA), junction of the oral-infraorbital canal to junction of the oral and angular canal; preopercular to main trunk (OTM), junction of the oral-infraorbital canal to junction of the main trunk-infraorbital canal; orbital canal length (OCL), junction of main trunk canal-infraorbital to junction of supratemporal-postorbital canals; supratemporal canal length (STL), from its junctions with the postorbital canal on either side of the head; spine to supratemporal canal (SPS), distance from anterior base of spine to the center of the supratemporal canal.

\section{Appendix B}

Locality, tissue identification number, voucher specimen collection number, and GenBank accession number for samples used in genetic analysis. Ind. = Indian Ocean; Atl. = Atlantic Ocean; Pac. $=$ Pacific Ocean; SWIO = Southwestern Indian Ocean; * = holotype.

\begin{tabular}{|c|c|c|c|}
\hline Locality & Tissue ID & $\begin{array}{c}\text { Voucher } \\
\text { Collection No. }\end{array}$ & $\begin{array}{c}\text { GenBank } \\
\text { Accession No. }\end{array}$ \\
\hline \multicolumn{4}{|l|}{ Chimaera willwatchi } \\
\hline Ind., Walters Shoal & GN11498 & Not accessioned & KX761229 \\
\hline Ind., Walters Shoal & GN11550 & CAS 242346 & KX761218 \\
\hline Ind., Walters Shoal & GN11711 & CAS 242358 & KX761223 \\
\hline Ind., Walters Shoal & GN11742 & CAS 242358 & KX761207 \\
\hline Ind., Walters Shoal & GN11753 & CAS 242342 & KX761211 \\
\hline Ind., Walters Shoal & GN11756 & CAS 242358 & KX761225 \\
\hline Ind., Walters Shoal & GN11808 & CAS 242346 & KX761195 \\
\hline Ind., Walters Shoal & GN11741 & CAS 242340 & KX761219 \\
\hline Ind., Walters Shoal & GN11543 & CAS 242356 & KX761198 \\
\hline Ind., Walters Shoal & GN15528 & CAS 242353 & KX761199 \\
\hline Ind., Walters Shoal & GN15526 & CAS 242347 & KX761217 \\
\hline Ind., Walters Shoal & GN15527 & CAS 242353 & KX761201 \\
\hline Ind., Walters Shoal & GN15529 & CAS 242353 & KX761214 \\
\hline Ind., SWIO Ridge & GN11712 & Not accessioned & KX761192 \\
\hline Ind., SWIO Ridge & GN11871 & CAS 242345 & KX761227 \\
\hline
\end{tabular}




\begin{tabular}{|c|c|c|c|}
\hline Ind., SWIO Ridge & GN15521 & CAS 242357 & KX761206 \\
\hline Ind., SWIO Ridge & GN11666 & CAS 242344 & KX761226 \\
\hline Ind., SWIO Ridge & GN11668 & CAS 242343 & KX761212 \\
\hline Ind., SWIO Ridge & GN11670 & Not accessioned & KX761222 \\
\hline Ind., SWIO Ridge & GN11672 & CAS 242343 & KX761202 \\
\hline Ind., SWIO Ridge & GN11533 & Not accessioned & KX761231 \\
\hline Ind., SWIO Ridge & GN11548 & CAS 242358 & KX761209 \\
\hline Ind., SWIO Ridge & GN11532 & MCZ 171972 & KX761200 \\
\hline Ind., N Madagascar Ridge & GN15531 & CAS 242354 & KX761220 \\
\hline Ind., N Madagascar Ridge & GN15532 & CAS 242354 & KX761224 \\
\hline Ind., N Madagascar Ridge & GN15533 & CAS 242354 & KX761213 \\
\hline Ind., N Madagascar Ridge & GN15534 & CAS 242354 & KX761210 \\
\hline Ind., SWIO Ridge & GN11822 & Not accessioned & KX761230 \\
\hline Ind., SWIO Ridge & GN11827 & Not accessioned & KX761196 \\
\hline *Ind., SWIO Ridge & GN15522 & CAS 242336 & KX761221 \\
\hline Ind., SWIO Ridge & GN15523 & CAS 242338 & KX761228 \\
\hline Ind., SWIO Ridge & GN15524 & CAS 242349 & KX761194 \\
\hline Ind., SWIO Ridge & GN15525 & CAS 242348 & KX761205 \\
\hline Ind., SWIO Ridge & GN15530 & CAS 242351 & KX761208 \\
\hline SW Indian Ocean & GN10954 & CSIRO H 5371-01 & KX761197 \\
\hline SW Indian Ocean & GN10953 & CSIRO H 5356-01 & KX761216 \\
\hline \multicolumn{4}{|l|}{ Chimaera diderae } \\
\hline $\begin{array}{l}\text { *Ind., N Madagascar } \\
\text { Ridge }\end{array}$ & GN11724 & CAS 242334 & KX761215 \\
\hline \multicolumn{4}{|l|}{ Chimaera buccanigella } \\
\hline *Ind., Walters Shoal & GN11492 & CAS 242335 & KX761203 \\
\hline Ind., N Madagascar Ridge & GN16034 & MNHN 2004-0818 & KX761204 \\
\hline $\begin{array}{l}\text { Ind., N Madagascar } \\
\text { Ridge }\end{array}$ & GN16035 & MNHN 2004-0819 & KX761191 \\
\hline \multicolumn{4}{|l|}{ Chimaera notafricana } \\
\hline Atl., South Africa & GN 14838 & - & KU163634 \\
\hline Atl., South Africa & GN 16916 & - & KU163635 \\
\hline \multicolumn{4}{|l|}{ Chimaera macrospina } \\
\hline Ind., Australia & GN 10955 & CSIRO H 6417-02 & KU163640 \\
\hline Pac., Australia & GN 10956 & CSIRO H 1382-02 & KU163646 \\
\hline \multicolumn{4}{|l|}{ Chimaera carophila } \\
\hline Pac., New Zealand & GN 12992 & NMNZ P.045580 & KU163637 \\
\hline Pac., New Zealand & GN 12993 & NMNZ P.040174 & KU163649 \\
\hline \multicolumn{4}{|l|}{ Chimaera opalescens } \\
\hline Atl., Europe & GN 13522 & MNHN-IC 2007-1557 & KU163647 \\
\hline Atl., Europe & GN 13524 & MNHN-IC 2007-1567 & KU163644 \\
\hline \multicolumn{4}{|l|}{ Chimaera lignaria } \\
\hline Pac., Tasmania & GN 10958 & CSIRO H 5125-01 & KU163639 \\
\hline Pac., Tasmania & GN 10959 & CSIRO H 4873-04 & KU163653 \\
\hline \multicolumn{4}{|l|}{ Chimaera obscura } \\
\hline Pac., New South Wales & GN10957 & CSIRO H 1383-02 & KX761193 \\
\hline \multicolumn{4}{|l|}{ Hydrolagus africanus } \\
\hline Atl., South Africa & GN16938 & CAS 241488 & KU934287 \\
\hline Atl., South Africa & GN16939 & CAS 241488 & KU934286 \\
\hline Atl., South Africa & GN16940 & USNM 438933 & KU934285 \\
\hline
\end{tabular}




\section{Appendix C}

Comparative Material. Chimaera bahamaensis - Holotype - FMNH 166362, mature female, $881 \mathrm{~mm}$ total length, TL, $528 \mathrm{~mm}$ body length, BDL; Atlantic Ocean, Tongue of the Ocean, Bahamas, Andros Island $\left(24^{\circ} 30^{\prime} 21^{\prime \prime} \mathrm{N}, 7^{\circ} 22^{\prime} 12^{\prime \prime} \mathrm{W}\right)$. Collected by Quinn et al., field number CI144, at 1483- 1506 m on 3 February 1974.

Chimaera cubana - 13 specimens - Holotype, MCZ 1464, , mature male, 728 mm TL, 427 mm BDL, Cuba, Matanzas Bay; FMNH 71595, female, 283 mm TL, 118.3 mm BDL, Puerto Rico, $18^{\circ} 16^{\prime} \mathrm{N}, 67^{\circ} 16.5^{\prime} \mathrm{W}$; USNM 222711, female, $429 \mathrm{~mm} \mathrm{TL}, 211 \mathrm{~mm}$ BDL, Caribbean Sea, $16^{\circ} 45^{\prime} \mathrm{N}, 81^{\circ} 27^{\prime} \mathrm{W}, 0-150 \mathrm{fms}$; USNM 222800, female, $300 \mathrm{~mm}$ TL, 115.4 mm BDL, Caribbean Sea, $15^{\circ} 38^{\prime} \mathrm{N}, 61^{\circ} 51^{\prime} \mathrm{W}, 0-245 \mathrm{fms}$; USNM 222800, female, $406 \mathrm{~mm}$ TL, 171 mm BDL, Caribbean Sea, $15^{\circ} 38^{\prime} \mathrm{N}, 61^{\circ} 15^{\prime} \mathrm{W}$, 0-245fms; MCZ 1385, male, 664 mm TL, 319 mm BDL, Cuba; MCZ 40682, male, 277 mm TL, 110.6 mm BDL, Caribbean Sea, $18^{\circ} 16^{\prime} \mathrm{N}, 67^{\circ} 17^{\prime} \mathrm{W}, 250 \mathrm{fms} ; \mathrm{MCZ} 40682$, male, $258 \mathrm{~mm}$ TL, $116.1 \mathrm{~mm}$ BDL, Caribbean Sea, $18^{\circ} 16^{\prime} \mathrm{N}, 67^{\circ} 17^{\prime} \mathrm{W}, 250 \mathrm{fms}$; USNM 222796, male, $549 \mathrm{~mm}$ TL, $241 \mathrm{~mm}$ BDL, Western Atlantic; USNM 222800, male, 267 mm TL, 129.1 mm BDL, L. Antilles, $15^{\circ} 38^{\prime} \mathrm{N}, 61^{\circ} 15^{\prime} \mathrm{W}, 0-245 \mathrm{fms} ;$ USNM 222800, male, $368 \mathrm{~mm}$ TL, $164 \mathrm{~mm}$ BDL, L. Antilles, $15^{\circ} 38^{\prime} \mathrm{N}, 61^{\circ} 15^{\prime} \mathrm{W}, 0-245 \mathrm{fms}$; USNM 222800, male, $215 \mathrm{~mm}$ TL, 109 mm BDL, L. Antilles, $15^{\circ} 38^{\prime} \mathrm{N}, 61^{\circ} 15^{\prime} \mathrm{W}, 0-245 \mathrm{fms}$; USNM 372728, immature male, $685 \mathrm{~mm}$ TL, 343 mm BDL, Puerto Rico, La Parguera, 180m.

Chimaera jordani, AMNH 4726, 700 mm TL, 483 mm BDL, male.

Chimaera monstrosa - 23 specimens - USNM 17492, mature male, 800 mm TL, 400 mm BDL, Atlantic Ocean (Norway); MNHN 21-137, mature male, 919 mm TL, 419 mm BDL, 
Atlantic Ocean (Cotes d'Espagne); MNHN 3, mature male, 906 mm TL, 403 mm BDL, Atlantic Ocean (Scotland), 58 40’ N, 9³0’W, $600 \mathrm{~m}$; MNHN 13, mature male, $655 \mathrm{~mm}$

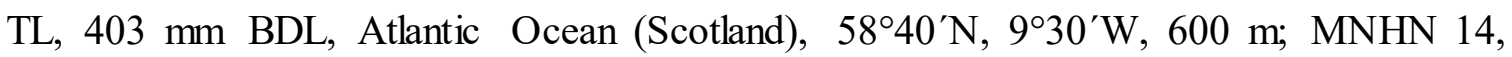
mature male, $845 \mathrm{~mm} \mathrm{TL}, 432 \mathrm{~mm}$ BDL, Atlantic Ocean (Scotland), 58 $40^{\prime} \mathrm{N}, 9^{\circ} 30^{\prime} \mathrm{W}$, 600 m; MNHN 16, mature male, 712 mm TL, 409 mm BDL, Atlantic Ocean (Scotland), $58^{\circ} 40^{\prime} \mathrm{N}, 9^{\circ} 30^{\prime} \mathrm{W}, 600 \mathrm{~m}$; MNHN 17, mature male, $795 \mathrm{~mm}$ TL, $414 \mathrm{~mm}$ BDL, Atlantic Ocean (Scotland), 58 40’N, 9³0’W, $600 \mathrm{~m}$; MNHN 18, mature male, $850 \mathrm{~mm}$ TL, 418

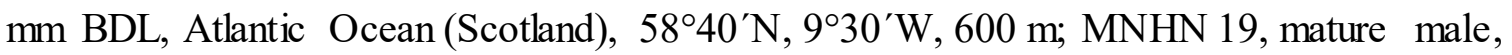
$826 \mathrm{~mm}$ TL, $430 \mathrm{~mm}$ BDL, Atlantic Ocean (Scotland), 5840’N, 9³0’W, $600 \mathrm{~m}$; MNHN 20, mature male, $688 \mathrm{~mm} \mathrm{TL}, 403 \mathrm{~mm}$ BDL, Atlantic Ocean(Scotland), 58 $40^{\prime} \mathrm{N}, 9^{\circ} 30^{\prime} \mathrm{W}$, 600 m; MNHN 24, mature male, 864 mm TL, 420 mm BDL, Atlantic Ocean (Scotland), $58^{\circ} 40^{\prime} \mathrm{N}, 9^{\circ} 30^{\prime} \mathrm{W}, 600 \mathrm{~m}$; MCZ 326, mature female, $725 \mathrm{~mm} \mathrm{TL}, 462 \mathrm{~mm}$ BDL, no data; MCZ 855, mature female, 774 mm TL, 449 mm BDL, Atlantic Ocean; USNM 10234, mature female, 708 mm TL, 453 mm BDL, Atlantic Ocean (Norway); MNHN 1, mature

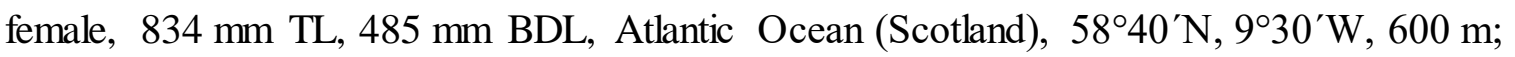
MNHN 8, mature female, 817 mm TL, 423 mm BDL, Atlantic Ocean (Scotland), $58^{\circ} 40^{\prime} \mathrm{N}$, 9³0’W, 600 m; MNHN 9, mature female, 916 mm TL, 488 mm BDL, Atlantic Ocean (Scotland), 58 $40^{\prime} \mathrm{N}, 9^{\circ} 30^{\prime} \mathrm{W}, 600 \mathrm{~m}$; MNHN 10, mature female, $902 \mathrm{~mm}$ TL, $421 \mathrm{~mm}$ BDL, Atlantic Ocean (Scotland), 58 $40^{\prime} \mathrm{N}, 9^{\circ} 30^{\prime} \mathrm{W}, 600 \mathrm{~m}$; MNHN 11, mature female, 980 mm TL, 469 mm BDL, Atlantic Ocean (Scotland), 5840’N, 9³0’W, 600 m; MNHN 12, mature female, $895 \mathrm{~mm} \mathrm{TL}, 430 \mathrm{~mm}$ BDL, Atlantic Ocean (Scotland), 58 $40^{\prime} \mathrm{N}$, 9³0’W, $600 \mathrm{~m}$; MNHN 21, mature female, $830 \mathrm{~mm}$ TL, $408 \mathrm{~mm}$ BDL, Atlantic Ocean 
(Scotland), 58 $40^{\prime} \mathrm{N}, 9^{\circ} 30^{\prime} \mathrm{W}, 600 \mathrm{~m}$; MNHN 23, mature female, $830 \mathrm{~mm}$ TL, $427 \mathrm{~mm}$ BDL, Atlantic Ocean (Scotland), 58 40N $, 9^{\circ} 30^{\prime} \mathrm{W}, 600 \mathrm{~m}$; LJVC-0459, mature female, 850 mm TL, 541 mm BDL. Chimaera cf. monstrosa five specimens: SAM 34517, mature male, 837 mm TL, 509 mm BDL, Cape Agulhas, southern Africa, 3449'9”S, 2000'0"E; SAM 34428, mature male, $820 \mathrm{~mm}$ TL, $486 \mathrm{~mm}$ BDL, southern Africa, 3443'3"S, 18 $03^{\prime} 6^{\prime \prime} \mathrm{E}, 717 \mathrm{~m}$; SAIAB 27132, mature female, $930 \mathrm{~mm}$ TL, $517 \mathrm{~mm}$ BDL, southern Africa, $32^{\circ} 30^{\prime} 5^{\prime \prime} \mathrm{S}, 16^{\circ} 24^{\prime} 3^{\prime \prime} \mathrm{E}, 800 \mathrm{~m}$; SAIAB 27133, mature female, $925 \mathrm{~mm} \mathrm{TL}, 522$ mm BDL, southern Africa, 32³0'5"S, 16²4'3"E, 800 m; SAM 34429, mature female, $880 \mathrm{~mm} \mathrm{TL}, 534 \mathrm{~mm}$ BDL, southern Africa, 345''' $\mathrm{S}, 18^{\circ} 11^{\prime} 7^{\prime \prime} \mathrm{E}, 903 \mathrm{~m}$.

Chimaera notafricana - 10 specimens - SAIAB 34834, immature male, 346 TL, 159 m BDL; non-accessioned specimen, $816 \mathrm{~mm}$ TL, 575 mm BDL, fresh mature male; nonaccessioned specimen, 788+ mm TL, 579 m BDL, fresh mature male; non-accessioned specimen, 850+ mm TL, $580 \mathrm{~mm}$ BDL, fresh mature male; accessioned specimen, 920 mm TL, 626 mm BDL, fresh mature male; non-accessioned specimen, 839 mm TL, 586 mm BDL, fresh mature male; non-accessioned specimen, $821 \mathrm{~mm}$ TL, $561 \mathrm{~mm}$ BDL, mature male; SAM 34551, 825 TL, 477 mm BDL, immature male; SAM 34423, 755+ mm TL, 470 mm BDL, mature male; SAM 27135, 812 mm TL, 497 mm BDL, immature male; SAM 27134, 878 mm TL, 513 mm BDL, female.

Chimaera opalescens - 2 specimens - USNM 390767 mature male, 916/686 mm, 2797 g,

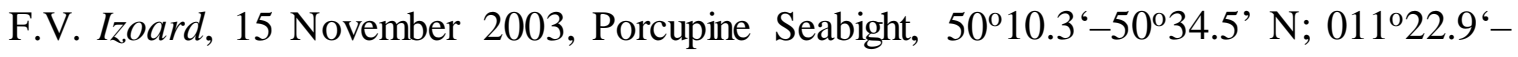
011²19.3‘ W, 1065-1300 m; USNM 390768, mature female, 1098/726 mm, 3428 g; F.V. 


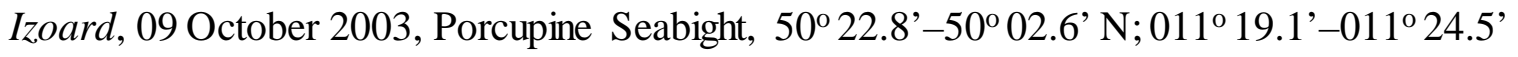
W, 950-1100 m.

Chimaera phantasma, SAM 39655, 470+ mm TL, 183 mm BDL, immature male; SAM 39655, 320 mm TL, 129 mm BDL, immature male.

Hydrolagus africanus - 6 specimens - SAIAB 014040, 620 mm TL, 293 mm BDL, female, Kenya; SAIAB 25211, immature male, 790 mm TL, 325 mm BDL: SAIAB 25712, immature male, 443 mm TL, 304 mm BDL: SAIAB 25730, immature male, 387+ TL, 290 mm BDL; SAM 33297, 800+ mm TL, 597 mm BDL, mature male: SAM 33297, 935 mm TL, 613 mm BDL, mature male. 